\title{
Phasenbildung, Phasenübergang und mechanische Eigenschaften des Funktionsmaterials Eisen-Palladium
}

\author{
Dissertation \\ zur Erlangung des mathematisch-naturwissenschaftlichen Doktorgrades \\ „Doctor rerum naturalium“ \\ der Georg-August-Universität Göttingen
}

vorgelegt von

Iris Kock

aus Überlingen

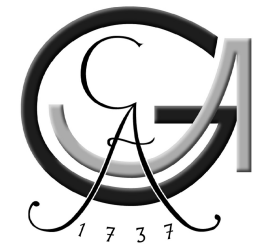

Göttingen, 2010 
Referent

Korreferent
Prof. Dr. Stefan G. Mayr

Prof. Dr. Reiner Kirchheim

Tag der mündlichen Prüfung: $\quad$ 12.07.2010 


\section{Inhaltsverzeichnis}

1 Einleitung 1

2 Grundlagen 5

2.1 Der martensitische Phasenübergang . . . . . . . . . . . 5

2.2 Pseudoelastizität und Pseudoplastizität . . . . . . . . . . 8

2.3 Der thermisch induzierte Formgedächtniseffekt . . . . . . . . . . . . . . 10

2.4 Der magnetische Formgedächtniseffekt . . . . . . . . . . . . . . 11

2.5 Magnetostriktion . . . . . . . . . . . . . . . . . . 12

2.6 Der Invar-Effekt . . . . . . . . . . . . . . . . . . . . . . . . . . . . . . 13

2.7 Die Legierung Eisen-Palladium _ . . . . . . . . . . . . . . . . . . . 13

2.8 Ternäre Systeme . . . . . . . . . . . . . . . . . . . . . . . . 18

3 Experimentelle Methoden $\quad 21$

3.1 Probenpräparation . . . . . . . . . . . . . . . . . . . . . . 21

3.2 Charakterisierungsmethoden .................... 23

3.2.1 Strukturbestimmung . . . . . . . . . . . . . 23

3.2.2 Messung der Magnetisierung . . . . . . . . . . . . 25

3.2 .3 Widerstandsmessung . . . . . . . . . . . . . 26

3.2.4 Untersuchung der mechanischen Eigenschaften . . . . . . . . . . 26

3.3 Das Vibrating Reed . . . . . . . . . . . . . . . . . . . . . . . 27

$\begin{array}{lll}4 & \text { Ergebnisse } & 39\end{array}$

4.1 Makroskopische und mikroskopische Struktur . . . . . . . . . . . . . . . 39

4.2 Phasen und ihre Stabilität . . . . . . . . . . . . . . . . . . . . 43

4.2.1 Binäre Phasen bei Zimmertemperatur . . . . . . . . . . . 43

4.2.2 Einfluss der Glühbehandlung . . . . . . . . . . . . . 44

4.2 .3 Zugabe von Kupfer . . . . . . . . . . . . . . . . . 46 
4.2.4 Mechanische Stabilität der Kristallstruktur . . . . . . . . . . . . 48

4.3 Der martensitische Phasenübergang . . . . . . . . . . . . . . . . . . . . 51

4.3 .1 Kristallstruktur . . . . . . . . . . . . . . . . . 51

4.3 .2 Widerstand . . . . . . . . . . . . . . . . 58

4.3 .3 Magnetisierung . . . . . . . . . . . . . . 6 60

4.3.4 Mechanische Eigenschaften . . . . . . . . . . . 63

5 Diskussion der Ergebnisse $\quad 71$

5.1 Stabilität gegen Entmischen . . . . . . . . . . . . . 71

5.2 Phasen bei Zimmertemperatur . . . . . . . . . . . . . . . 75

5.3 Mechanische Eigenschaften . . . . . . . . . . . . . . . . . . . 79

5.3.1 Spannungsinduzierte Dehnung . . . . . . . . . . . . . . . . 80

5.3.2 Magnetfeldinduzierte Dehnung . . . . . . . . . . . . . . . . 83

5.3.3 Aufweichen des Kristallgitters beim Phasenübergang . . . . . . 83

5.3.4 Vergleich der Elastizitätsmoduli . . . . . . . . . . . . . . 85

5.3 .5 Dämpfung . . . . . . . . . . . . . . . . . 87

5.4 Der martensitische Phasenübergang . . . . . . . . . . . . . . . . 90

5.4.1 Änderung der Magnetisierung . . . . . . . . . . . . . . . . 90

5.4 .2 Änderung des Widerstands . . . . . . . . . . . . . . . . . . 91

5.4.3 Einfluss der Mikrostruktur auf den Phasenübergang . . . . . . . 92

$\begin{array}{lll}6 & \text { Zusammenfassung } & 99\end{array}$

$\begin{array}{ll}\text { Literaturverzeichnis } & 103\end{array}$

$\begin{array}{ll}\text { Danksagung } & 113\end{array}$ 


\section{Kapitel 1}

\section{Einleitung}

Große Fortschritte in der modernen Technik basieren neben der Miniaturisierung von Bauteilen auf dem gezielten Einsatz von Funktionsmaterialien. Besonders in der Sensorik und Aktorik eröffnen diese Smart Materials, deren physikalische Eigenschaften durch Veränderung der Umgebungsbedingungen gezielt beeinflusst werden können, viele technische Möglichkeiten, indem sie z. B. den Einsatz aufwendiger Regel- und Steuerkreise oder mechanischer Stellelemente überflüssig machen.

Die Legierung Eisen-Palladium ist ein besonders interessantes Funktionsmaterial, da ihre physikalischen Eigenschaften auf vielfältige Weise beeinflusst werden können. So zeigt das Material beim Anlegen eines Magnetfeldes eine deutliche Längenänderung, die als Magnetostriktion bezeichnet wird [1]. Außerdem kann an diesem Material der Invar-Effekt beobachtet werden [2], d. h. in einem weiten Temperaturbereich zeigt es nahezu keine thermische Ausdehnung [3, 4]. Bei einer geeigneten Zusammensetzung im Bereich $\mathrm{Fe}_{70} \mathrm{Pd}_{30}$ durchläuft das Material einen martensitischen Phasenübergang von der kubisch flächenzentrierten Hochtemperaturphase, dem Austenit, in eine tetragonal flächenzentrierte Tieftemperaturphase, den Martensit [3]. Die Übergangstemperatur liegt etwas unterhalb von Zimmertemperatur [5] und kann durch leichte Variation der Zusammensetzung stark verändert werden [3, 6]. Dies ermöglicht wahlweise pseudoplastisches oder pseudoelastisches Verhalten des Materials, d. h. es kann mit geringen Kräften sehr stark gedehnt werden. Bei Temperaturen unterhalb der Umwandlungstemperatur bleibt diese Dehnung nach dem Entlasten bestehen, oberhalb der Umwandlungstemperatur bildet sie sich wieder zurück. Auf diesem Verhalten basiert der thermische Formgedächtniseffekt [7, 8]: Wird das Material unterhalb der Umwandlungstemperatur verformt und anschließend über die Übergangstemperatur erwärmt, so bildet sich die Deformation zurück, und das Material erinnert sich an seine ursprüngliche Form. Befindet sich das Material in der Martensitphase, kann außerdem der magnetische Formgedächtniseffekt auftreten [9]. Dabei wird eine makroskopische Formänderung induziert, indem die verschiedenen Varianten der tetragonal verzerrten Einheitszelle in einem externen Magnetfeld ausgerichtet werden [10, 11].

Die meisten der soeben beschriebenen Effekte können besonders gut an einkristallinem Material beobachtet werden, das entsprechend seit langem intensiv untersucht wird [1, 5, 6, 12-18]. Die Herstellung von Einkristallen ist jedoch aufwendig und teuer, insbesondere da die Legierung bei langsamem Abkühlen in $\alpha$-Eisen und eine geordnete 
$\mathrm{Fe}_{50} \mathrm{Pd}_{50}$-Phase entmischt $[19,20]$. Auch wenn kürzlich bei der Herstellung freistehender einkristalliner Filme mit einer Dicke von mehreren hundert Nanometern beachtliche Fortschritte erzielt wurden [21], bleibt die Präparation von Schichten für den Einsatz in miniaturisierten Bauteilen schwierig und zeitintensiv. Sehr viel einfacher und schneller ist die Herstellung polykristalliner Folien durch rasches Abschrecken des Materials aus der flüssigen Phase. Hier bietet sich beispielsweise die Technik des Splatquenching an, die ursprünglich zur Herstellung metallischer Gläser entwickelt wurde [22, 23]. Beim Abschrecken des flüssigen Materials zwischen zwei Kupferstempeln entstehen polykristalline Folien mit einer Dicke von etwa $50 \mu \mathrm{m}$ [24].

Ein Vergleich der Arbeiten von Matsui et al. und Cui et al. zeigt, dass Einkristalle und Polykristalle bei gleicher Zusammensetzung in unterschiedlichen Phasen vorliegen $[3,6]$. Daher können die Erkenntnisse zur Bildung und Stabilität der Phasen, die an Einkristallen gewonnen wurden, nicht ohne Weiteres auf Polykristalle übertragen werden. Deshalb ist es zunächst wichtig, Erkenntnisse über die Stabilität der Splats gegenüber der Bildung von Ausscheidungen oder einer unerwünschten raumzentrierten Phase zu gewinnen. Dazu werden Splats verschiedener Zusammensetzung hergestellt und die Auswirkungen verschiedener Glühbehandlungen auf ihre Kristallstruktur untersucht.

Da einige der speziellen Effekte der Legierung Eisen-Palladium wie der magnetische Formgedächtniseffekt nur in der Martensitphase auftreten können, ist eine hohe Übergangstemperatur wünschenswert, so dass das Material bei Zimmertemperatur zuverlässig in der Martensitphase vorliegt. In einem binären System sind jedoch die Möglichkeiten zu derartiger Optimierung, beispielsweise durch Variation der Zusammensetzung, beschränkt. Daher bietet es sich an, ein drittes Element hinzuzulegieren, da ternäre Systeme exzellente Möglichkeiten bieten, verschiedene Parameter wie beispielsweise Eisengehalt und Valenzelektronenkonzentration unabhängig voneinander zu variieren. So ist es möglich, diejenigen Faktoren zu identifizieren, von denen Phasenstabilität und Übergangstemperatur primär abhängen, und diese gezielt zu beeinflussen. Daher wird in dieser Arbeit, basierend auf Ergebnissen von Hamann et al. [25], Kupfer als drittes Element zulegiert und die Auswirkungen auf die Stabilität der Austenit- und Martensitphase und die Transformationstemperatur untersucht. Anhand dieser Untersuchungen soll der Zusammenhang von Eisengehalt, Valenzelektronenkonzentration, Phasenstabilität und Transformationstemperatur identifiziert werden, um eine gezielte Optimierung der Legierung zu ermöglichen.

Das unterschiedliche Verhalten von Einkristallen und Polykristallen zeigt, dass viele Eigenschaften der Legierung Eisen-Palladium erheblich durch die Mikrostruktur des Materials beeinflusst werden. Deshalb liegt ein Schwerpunkt dieser Arbeit auf der Untersuchung des Einflusses der Mikrostruktur auf den martensitischen Phasenübergang. Durch Glühen bei verschiedenen Temperaturen wird die Mikrostruktur der Splats modifiziert, so dass auch in binären Proben ein verbessertes Transformationsverhalten erreicht werden kann.

Auch die mechanischen Eigenschaften der Splats wie Elastizitätsmodul und Dämpfung, deren Kenntnis für den Einsatz in Aktoren besonders wichtig ist, können sich grundsätzlich von denen einkristallinen Materials unterscheiden. Daher werden in dieser Arbeit auch die mechanischen Eigenschaften und ihre Änderung während des martensitischen Phasenübergangs charakterisiert. Zu diesem Zweck wurde ein eigens für die Unter- 
suchung von Splats konzipiertes Vibrating-Reed-Experiment aufgebaut. Ein schmaler Probenstreifen wird an einer Seite fest eingespannt und kapazitiv zu Schwingungen nahe seiner Eigenfrequenz angeregt, die mit Hilfe eines Lichtzeigers detektiert werden. Aus der abklingenden freien Schwingung nach Abschalten der Anregung können Elastizitätsmodul und Dämpfung gewonnen werden. Die Probentemperatur kann in dem für Eisen-Palladium besonders interessanten Temperaturbereich zwischen $-70{ }^{\circ} \mathrm{C}$ und $150^{\circ} \mathrm{C}$ variiert werden, so dass die Veränderungen der mechanischen Eigenschaften während des Phasenüberganges beobachtet werden können. Anhand eines Vergleichs mit Spannungs-Dehnungsmessungen mit einer kommerziellen Zugprüfanlage und Röntgendiffraktogrammen gedehnter Proben können die besonderen mechanischen Eigenschaften der Splats erklärt werden. 


\section{Kapitel 2}

\section{Grundlagen}

Eisen-Palladium-basierte Legierungen mit einer Zusammensetzung nahe $\mathrm{Fe}_{70} \mathrm{Pd}_{30}$ gehören zu einer höchst interessanten Klasse von Funktionsmaterialien. Neben konventioneller Magnetostriktion [1] und dem Invar-Effekt [2] werden in diesen Legierungen auch der thermische [8] und der magnetische Formgedächtniseffekt [9] sowie pseudoelastisches und pseudoplastisches Verhalten beobachtet. Alle diese Effekte treten auf, da die Gitterstruktur des Materials in der Nähe des martensitischen Phasenüberganges stark aufweicht, während sich die elektronische Struktur und damit der magnetische Druck innerhalb des Materials stark ändern. Um die oben genannten Effekte und deren Zusammenhang mit dem martensitischen Phasenübergang besser zu verstehen, sollen in diesem Kapitel zunächst kurz martensitische Phasenübergänge, Formgedächtnis- und Invar-Effekt sowie Magnetostriktion vorgestellt werden, bevor die besonderen Eigenschaften Eisen-Palladium-basierter Legierungen erläutert werden.

\subsection{Der martensitische Phasenübergang}

Die Bezeichnung martensitische Phasenumwandlung geht auf den deutschen Metallurgen Adolf Martens zurück, der beim Härten von Stahl einen diffusionslosen Übergang von der kubisch flächenzentrierten Hochtemperaturphase, dem Austenit, in eine kubisch raumzentrierte oder tetragonal raumzentrierte Tieftemperaturphase, den Martensit, beobachtete [26]. Im Gegensatz zu den meisten anderen Phasenübergängen bewegen sich hier die Atome gemeinsam um den Bruchteil eines Atomabstandes, so dass eine eindeutige Beziehung zwischen dem Kristallgitter der austenitischen und der martensitischen Phase erhalten bleibt. Da derartige Umwandlungen auch bei vielen anderen Metallen und Legierungen beobachtet wurden, hat sich die Bezeichnung martensitische Umwandlung allgemein für diffusionslose Phasenübergänge mit kollektiver Atombewegung und fester Beziehung zwischen den Gittern der beiden Phasen verbreitet. Die Bezeichnungen Austenit und Martensit für die Hoch- bzw. Tieftemperaturphase werden entsprechend übernommen [27].

Im Allgemeinen ist die Umwandlung von Austenit zu Martensit ein exothermer Phasenübergang erster Ordnung [28]. Der Übergang wird jedoch nur induziert, wenn die Differenz der Gibbsschen Freien Enthalpie $\Delta G^{A-M}$ zwischen Austenit und Martensit 


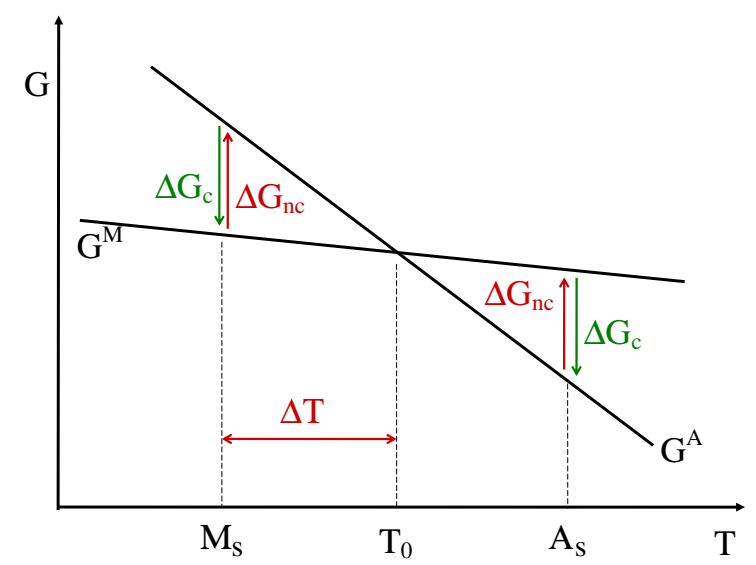

Abbildung 2.1: Die Martensitbildung beginnt erst, wenn die Unterkühlung $\Delta T$ so groß ist, dass die freie Enthalpie $\Delta G^{A-M}>0$ ist.

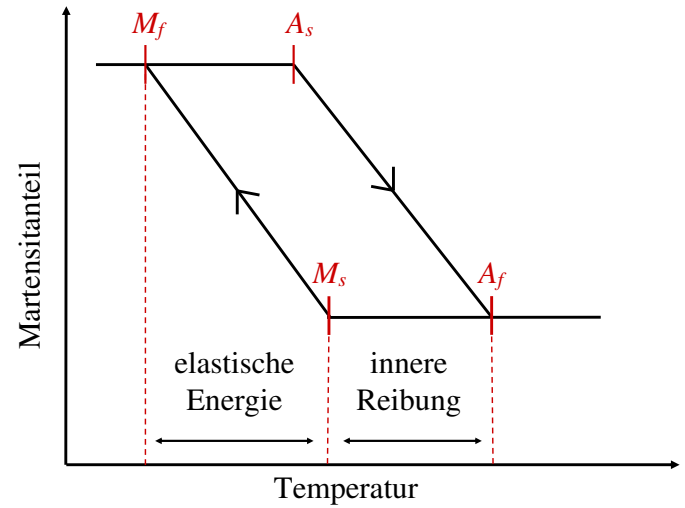

Abbildung 2.2: Die Breite der Hysterese hängt von der inneren Reibung und der gespeicherten elastischen Energie ab.

größer ist als die dabei aufzuwendende Energie zur elastischen Verzerrung des Kristallgitters $\Delta G_{\mathrm{el}}$, zur Bildung neuer Grenzflächen ${ }^{(1)} \Delta G_{\mathrm{s}}$ und als der Beitrag der inneren Reibung $\Delta G_{\text {if }}$, also

$$
\Delta G^{A-M}=\underbrace{\left(G^{A}-G^{M}\right)}_{\Delta G_{\mathrm{c}}}+\underbrace{\left(\Delta G_{\mathrm{el}}+\Delta G_{\mathrm{s}}+\Delta G_{\mathrm{if}}\right)}_{\Delta G_{\mathrm{nc}}}>0 .
$$

Dies ist in Abbildung 2.1 verdeutlicht. Bei martensitischen Phasenumwandlungen sind die nicht-chemischen Beiträge $\Delta G_{\mathrm{nc}}$ in der Regel von einer ähnlichen Größe wie die Änderung der chemischen freien Enthalpie $\Delta G_{c}$. Daher beginnt die Martensitbildung nicht bei der Gleichgewichtstemperatur $T_{0}$ sondern erst, wenn eine gewisse Unterkühlung $\Delta T$ erreicht ist. Entsprechendes gilt für die Austenitbildung, so dass die Phasenumwandlung eine Temperaturhysterese durchläuft, wie sie in Abbildung 2.2 schematisch dargestellt ist. Die Breite der Hysterese wird durch die nicht chemischen Beiträge $\Delta G_{\mathrm{nc}}=\Delta G_{\mathrm{el}}+\Delta G_{\mathrm{s}}+\Delta G_{\text {if }}$ zur Gibbsschen freien Enthalpie bestimmt. Die Differenz zwischen Martensitstarttemperatur $M_{s}$ und Austenitendtemperatur $A_{f}$ hängt von der inneren Reibung sowie den bei der Bewegung der Phasengrenze entstandenen Defekten und neu gebildeten Grenzflächen ab. Der Abstand von Martensitstart- und Martensitendtemperatur hingegen wird von der elastischen Energie $\Delta G_{\mathrm{el}}$ bestimmt. Diese wird gespeichert, wenn das Gitter während des Phasenüberganges elastisch verzerrt wird (Abbildung 2.2). Je weiter die Umwandlung fortschreitet, desto größer werden die Verzerrungsfelder, und umso größer muss die treibende Kraft und damit die Unterkühlung sein, damit die Transformation weiter fortschreitet. Bei der Rückumwandlung in den Austenit wird diese elastische Energie wieder freigesetzt und bewirkt, dass die Austenitbildung bereits bei einer niedrigeren Temperatur beginnt, so dass ebenfalls eine Differenz zwischen Austenitstarttemperatur $A_{s}$ und Austenitendtemperatur $A_{f}$ entsteht $[29,30]$. Martensit und Austenit können also über einen gewissen Temperaturbe-

\footnotetext{
${ }^{(1)}$ Im Folgenden beziehen sich die Indizes auf die englischen Bezeichnungen, z.B. $s$ für surface und if für internal friction.
} 
reich koexistieren. Der Volumenanteil des Martensits wächst während des Abkühlens, hängt jedoch nur von der Temperatur und nicht von der Zeit ab, die sich das Material bei dieser Temperatur befindet. Deshalb wird diese Art der Transformation auch als thermoelastische Umwandlung bezeichnet.

Der Martensit ist von geringerer Symmetrie als der Austenit, weshalb makroskopische und mikroskopische Dehnungen besser kompensiert werden können. Daher kann die Bildung von Martensit nicht nur durch Absenken der Temperatur, sondern auch durch Anlegen einer hinreichend großen mechanischen Spannung induziert werden. Der Zusammenhang zwischen der mechanischen Spannung $\sigma$ und dem Einsetzen der Martensitbildung kann mit einer Clausius-Clapeyron-Gleichung beschrieben werden:

$$
\frac{\mathrm{d} \sigma}{\mathrm{d} M_{s}}=-\frac{\Delta H}{T \epsilon}
$$

mit der latenten Wärme $\Delta H$, die bei der Transformation frei wird, der Temperatur $T$ und der transformationsinduzierten Dehnung $\epsilon$ in Richtung der angelegten Spannung.

Eine kristallographische Beschreibung des martensitischen Phasenüberganges kann in drei Schritten erfolgen, die allerdings nicht notwendigerweise die realen Atombewegungen wiedergeben $[31,32]$ :

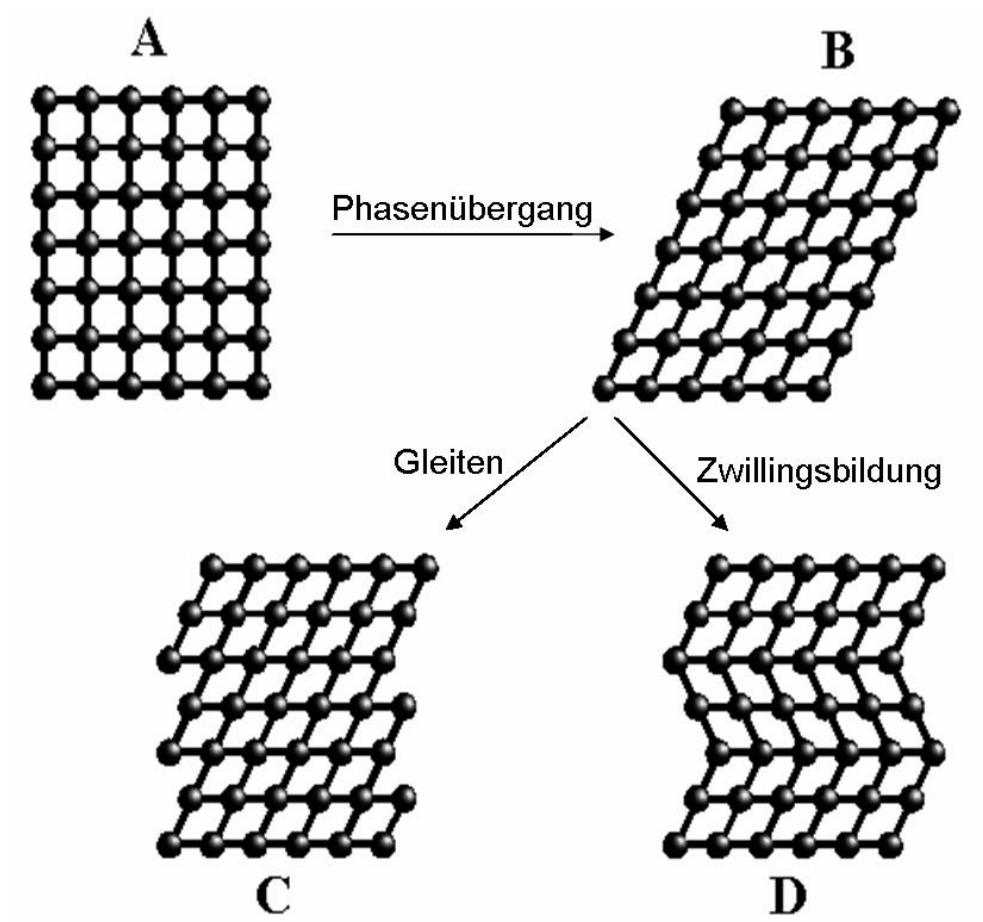

Abbildung 2.3: Phasenübergang von Austenit zu Martensit [33]

- gittervariante Scherung: Durch eine kollektive Bewegung der Atome wird das Kristallgitter in die martensitische Struktur überführt. Dabei wird das Gitter entlang einer neutralen Habitusebene geschert (Abbildung 2.3 A $\rightarrow$ B). Dieser Vorgang wird als Bainsche Verformung bezeichnet. Dabei wird die austenitische Matrix stark verzerrt. 
- gitterinvariante Scherung: Durch eine gitterinvariante Scherung wird die makroskopische Form des Martensits der austenitischen Matrix angepasst und so deren Verzerrung minimiert. Dies kann entweder durch Gleiten oder durch Zwillingsbildung geschehen (Abbildung 2.3 B $\rightarrow$ C bzw. D). Scherung durch Gleiten ist ein irreversibler Prozess, bei dem einzelne atomare Bindungen aufgebrochen werden [28]. In vielen Fällen, insbesondere in geordneten Legierungen, ist dies energetisch ungünstiger als die Bildung von Zwillingsgrenzen, da diese die atomaren Bindungsverhältnisse und damit auch Ordnung erhält [28]. An einer Zwillingsgrenze treffen zwei Martensitvarianten aufeinander, die sich nur durch die Orientierung ihrer Kristallstruktur unterscheiden, Zwillingsgrenzen sind also Spiegelebenen der Kristallstruktur. Die Anzahl und Art der Bindungen von Atomen der Zwillingsgrenze sind identisch zu denen von Atomen, die nicht zur Grenze gehören. Daher haben Zwillingsgrenzen in der Regel eine sehr geringe Energie, sind sehr mobil und die Stabilität der martensitischen Phase wird nicht wesentlich von der Anzahl der Zwillingsgrenzen beeinflusst. Die Verformung durch Zwillingsbildung ist reversibel und damit essentiell für das Auftreten des Formgedächtniseffektes.

- Rotation: In der Regel ist zusätzlich zu den oben genannten Scherprozessen noch eine Rotation des Gitters notwendig, um eine invariante Habitusebene zu erhalten.

Die martensitische Transformation ist also eine scherdominierte reversible Phasenumwandlung, bei der die Konfiguration der atomaren nächsten Nachbarn erhalten bleibt. Die oben genannten Scherprozesse, die zur martensitischen Umwandlung führen, werden durch ein anisotropes Aufweichen des Kristallgitters ermöglicht. Diese Scherinstabilität begründet sich bereits im Vorfeld des Phasenüberganges durch ein Aufweichen der Phononen-Dispersionsrelation [34]. Aufgrund dieser Eigenschaften ermöglicht die martensitische Transformation das Auftreten der eingangs erwähnten Effekte.

\subsection{Pseudoelastizität und Pseudoplastizität}

Materialien, die eine martensitische Phasenumwandlung durchlaufen, zeigen in der Nähe und unterhalb der Umwandlungstemperatur einen ungewöhnlichen Zusammenhang zwischen Spannung und Dehnung, der deutlich von dem linearen elastischen Verhalten anderer Metalle abweicht.

Oberhalb der Umwandlungstemperatur kann ein Verhalten beobachtet werden, das als Pseudoelastizität oder gelegentlich auch als Superelastizität bezeichnet wird. Bei diesen Temperaturen ist die Austenitphase stabil. Wird nun eine mechanische Spannung angelegt, beginnt das Material sich zunächst ähnlich einem konventionellen Metall mit steigender Spannung linear zu dehnen. Ab einer bestimmten (temperaturabhängigen) Spannung $\sigma_{M}$ beginnt die Bildung von Martensit gemäß Gleichung 2.2. Die Martensitvarianten orientieren sich dabei so, dass eine maximale Dehnung in Richtung der angelegten Spannung erfolgt. So können ohne nennenswerte Erhöhung der Spannung Dehnungen bis zu 10\% erreicht werden. Die maximal erreichbare Dehnung hängt dabei vom Achsenverhältnis der Einheitszelle des Martensits ab. In orthorhombischen 
Strukturen ist sie gegeben durch das Verhältnis der längsten Achse $a$ zur kürzesten Achse $c$ :

$$
\epsilon_{\max }=1-\frac{c}{a}
$$

Das so entstehende Martensitplateau ist in Abbildung 2.4(a) dargestellt. Wenn das gesamte Material in die martensitische Phase transformiert ist, steigt die zur weiteren Dehnung benötigte Spannung wieder linear an. Beim Entlasten retransformiert das Material in die austenitische Phase, so dass die gesamte Dehnung zurückgeht. Das Spannungs-Dehnungs-Verhalten ist also vollständig reversibel.

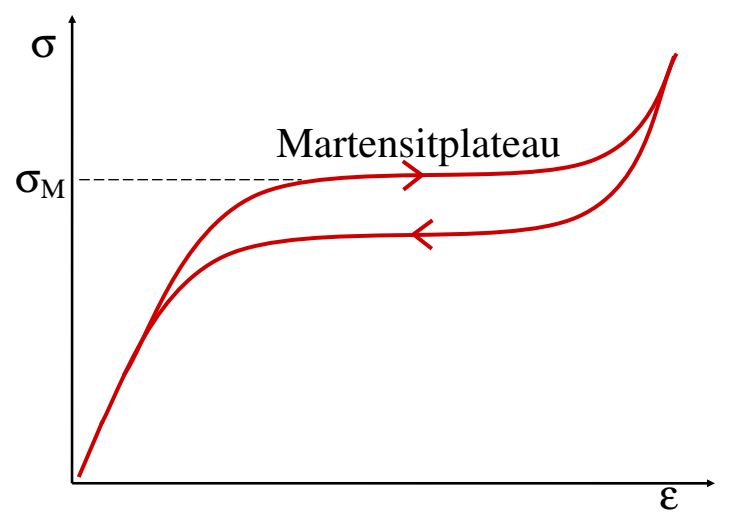

(a) Pseudoelastizität $\left(T>M_{s}\right)$

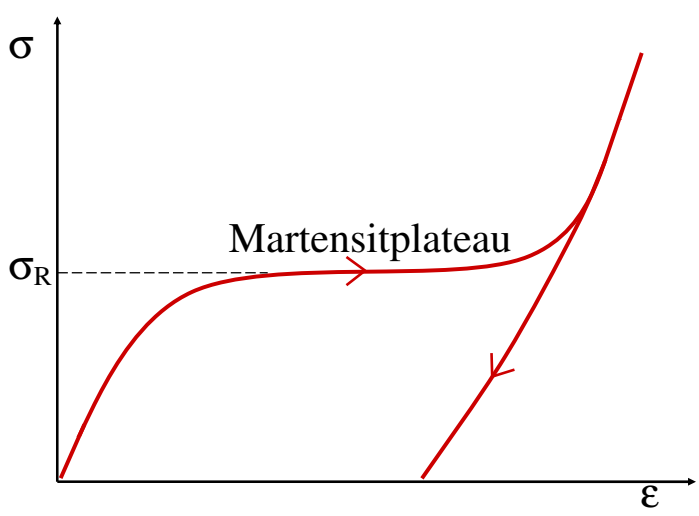

(b) Pseudoplastizität $\left(T<M_{f}\right)$

Abbildung 2.4: Durch Reorientierung der Martensitvarianten können Dehnungen bis $\mathrm{zu} 10 \%$ erreicht werden.

Bei niedrigeren Temperaturen, wenn das Material vollständig in der martensitischen Phase vorliegt, tritt pseudoplastisches Verhalten auf. Beim Anlegen einer mechanischen Spannung beginnt das Material wieder, sich zunächst linear auszudehnen. Ab einer gewissen Spannung $\sigma_{R}$ beginnen die Martensitvarianten, sich so auszurichten, dass die angelegte Spannung bestmöglich kompensiert wird. Im Spannungs-Dehnungs-Diagramm (Abbildung 2.4(b)) tritt wiederum ein Martensitplateau auf und es können sehr große Dehnungen ohne nennenswerte Erhöhung der Spannung erreicht werden. Wenn alle Varianten ausgerichtet sind, ist das Material vollständig entzwillingt, und die Spannung steigt wieder linear an. Beim Entlasten geht diese lineare Dehnung zurück. Im Gegensatz zum pseudoelastischen Verhalten bleibt die durch die Entzwillingung verursachte Dehnung jedoch bestehen, da keine treibende Kraft für die Rückorientierung der Martensitvarianten auftritt.

Erst wenn das Material über die Transformationstemperatur erwärmt wird und in die austenitische Phase übergeht, bildet sich die Dehnung zurück. Diese Reversibilität der Deformation wird als Formgedächtniseffekt bezeichnet und soll im folgenden Abschnitt genauer erläutert werden. 


\title{
2.3 Der thermisch induzierte Formgedächtniseffekt
}

Als thermischen Formgedächtniseffekt bezeichnet man die Fähigkeit bestimmter Materialien, nach einer Verformung unterhalb einer gewissen Temperatur beim anschließenden Erwärmen ihre ursprüngliche Form wieder anzunehmen [33]. Ermöglicht wird diese Formänderung durch die oben beschriebene thermoelastische reversible martensitische Phasenumwandlung.

Die Funktionsweise des thermisch induzierten Formgedächtniseffektes kann wie in Abbildung 2.5 schematisch in drei Schritten dargestellt werden:

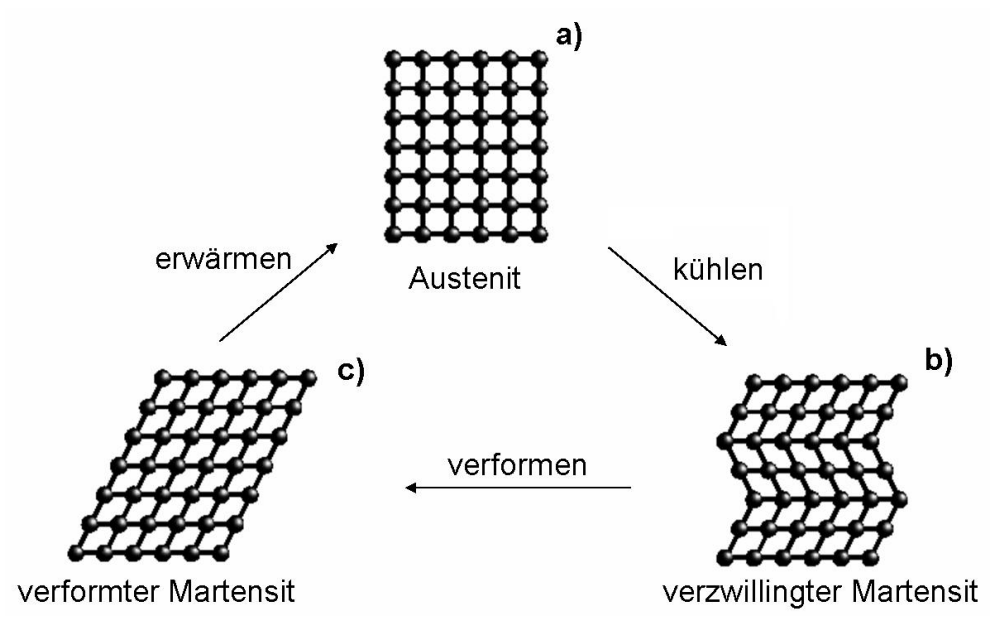

\begin{abstract}
Abbildung 2.5: Beim Kühlen transformiert der Austenit zu verzwillingtem Martensit, der durch Verschieben der Zwillingsgrenzen leicht verformt werden kann. Beim Erwärmen nimmt das Material wieder seine ursprüngliche Form an [33].
\end{abstract}

Zunächst liegt das Material in der austenitischen Hochtemperaturphase vor (Abbildung 2.5 a). Beim Kühlen unter die Übergangstemperatur bildet sich Martensit, der aufgrund der räumlichen Restriktionen die äußere Form des Austenits beibehalten muss, so dass Zwillinge gebildet werden (Abbildung 2.5 b). Die Zwillingsgrenzen sind hoch beweglich, weshalb der Martensit leicht verformbar ist. Wirkt nun eine äußere Spannung auf den Kristall, bilden sich bevorzugt diejenigen Martensitvarianten, die der Spannung am meisten nachgeben (Abbildung 2.5c). Dabei müssen keine atomaren Bindungen aufgebrochen werden, lediglich die Zwillingsgrenzen wandern durch den Kristall. Da die Nachbarschaftsbeziehungen zwischen den Atomen erhalten bleiben, nimmt der Kristall beim Erwärmen und der damit einhergehenden Phasenumwandlung zum hochsymmetrischen Austenit wieder seine ursprüngliche Form an.

Bei erneutem Abkühlen und Wiedererwärmen tritt die im Martensit induzierte Verformung nicht wieder auf, weshalb dieser Vorgang auch als Einwegeffekt bezeichnet wird. Durch eine spezielle thermische und mechanische Behandlung des Materials, ein sogenanntes Training, bei dem gezielt Versetzungen in die Struktur eingebaut werden, kann erreicht werden, dass sowohl beim Abkühlen als auch beim Erwärmen eine Formänderung auftritt. Ein solches Verhalten wird als Zweiwegeffekt bezeichnet [35]. 


\title{
2.4 Der magnetische Formgedächtniseffekt
}

In ferromagnetischen Formgedächtnislegierungen können zwei weitere Arten des Formgedächtniseffekts auftreten, die als magnetfeldinduzierter Martensit (MIM) bzw. magnetfeldinduzierte Reorientierung (MIR) bezeichnet werden. Das Prinzip des MIM ist dem des thermisch induzierten Formgedächtniseffektes sehr ähnlich. Der Unterschied besteht lediglich darin, dass der martensitische Phasenübergang nicht durch Änderung der Temperatur, sondern beim Anlegen eines Magnetfeldes durch einen Beitrag der Magnetisierung zur Freien Enthalpie $\Delta G_{M}$ induziert wird. Dieser Effekt wird beispielsweise in Nickel-Mangan-Indium-Legierungen beobachtet [36], deren Magnetisierung sich beim Phasenübergang entscheidend ändert.

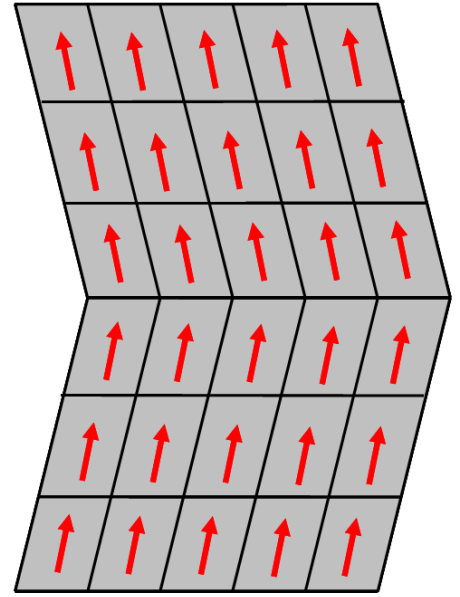

(a) ohne externes Magnetfeld

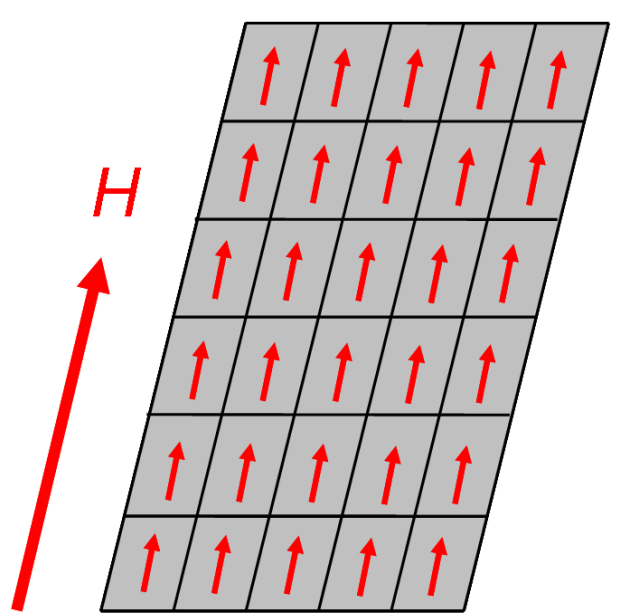

(b) mit externem Magnetfeld

\begin{abstract}
Abbildung 2.6: Beim Anlegen eines externen Magnetfeldes rotieren die magnetischen Momente in Feldrichtung. Ist die magnetische Anisotropie hinreichend groß, werden dabei die Martensitvarianten umorientiert.
\end{abstract}

In Eisen-Palladium-Legierungen hingegen tritt die magnetfeldinduzierte Reorientierung auf [9]. Dieser Effekt findet nur in der martensitischen Phase statt und kann nur in Materialien beobachtet werden, deren magnetische Anisotropieenergie größer ist als die Energie, die nötig ist, um Zwillingsgrenzen durch das Material zu bewegen [11]. In magnetisch anisotropem Material richten sich die magnetischen Momente vorzugsweise entlang einer bestimmten Achse der Einheitszelle aus, der magnetischen Vorzugsrichtung. Um die magnetischen Momente aus dieser Vorzugsrichtung herauszurotieren, muss die magnetische Anisotropieenergie überwunden werden. In Abwesenheit eines äußeren Magnetfeldes sind im unverformten polykristallinen Martensit alle Martensitvarianten und damit alle Orientierungen der magnetischen Momente möglich. In einem hinreichend starken Magnetfeld hingegen richten sich die magnetischen Momente entsprechend der Feldlinien aus. Wenn die Anisotropieenergie größer ist als die Energie, die nötig ist, um eine Zwillingsgrenze zu verschieben, wachsen diejenigen Martensitvarianten, deren Vorzugsrichtung mit dem angelegten Feld zusammenfällt. Diese Umorientierung der Martensitvarianten hat eine makroskopische Formänderung zur Folge [37], die in Abbildung 2.6 stark vereinfacht dargestellt ist. 
Für den praktischen Einsatz solcher Materialien ist es vorteilhaft, wenn sie bei Raumtemperatur martensitisch sind. Außerdem sind große magnetische Momente sowie eine hohe Beweglichkeit der Zwillingsgrenzen notwendig, da für eine Formänderung ein umso stärkeres Magnetfeld benötigt wird, je kleiner die magnetischen Momente und die Zwillingsbeweglichkeit sind.

\title{
2.5 Magnetostriktion
}

Unter Magnetostriktion versteht man allgemein die Längenänderung eines Materials im Magnetfeld. Sie tritt in fast allen ferromagnetischen Materialien auf und beruht auf einem grundsätzlich anderen Mechanismus als der magnetische Formgedächtniseffekt.

Auf mikroskopischer Skala hat die Magnetostriktion einen ähnlichen Ursprung wie die magnetokristalline Anisotropie. Eine spontane Dehnung des Kristallgitters kann dazu führen, dass die Anisotropieenergie stärker absinkt, als die elastische Energie steigt. So wird eine geringfügige Abweichung von der kubischen Symmetrie des Gitters begünstigt ${ }^{(2)}$. Insbesondere wenn das Kristallgitter sehr weich gegenüber mechanischer Verformung ist, kann es zu signifikanter Dehnung der Einheitszellen in Richtung der magnetischen Momente kommen.
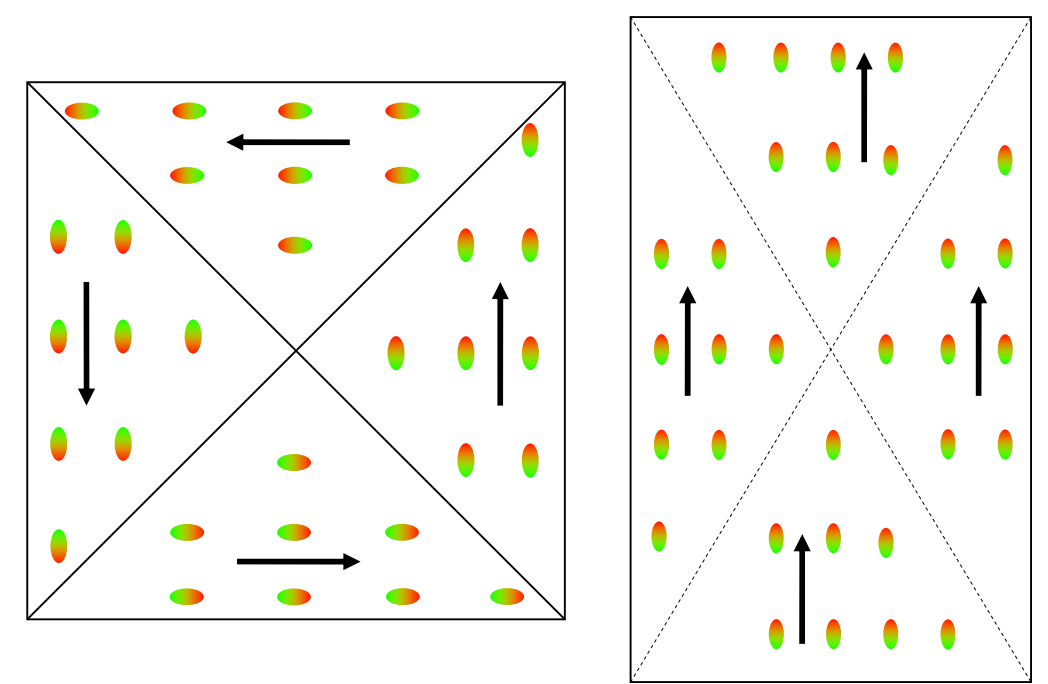

\begin{abstract}
Abbildung 2.7: Ohne externes Magnetfeld (links) heben sich die mikroskopischen Verzerrungen der Domänen gegenseitig auf. Erst wenn in einem externen Feld die magnetischen Domänen ausgerichtet werden und die magnetischen Momente in die gleiche Richtung orientiert sind, kommt es zu einer makroskopischen Dehnung (rechts).
\end{abstract}

In Abwesenheit eines externen Magnetfeldes sind die magnetischen Domänen so verteilt, dass die magnetostatische Energie minimiert wird. Daher heben sich die mikroskopischen Verzerrungen der Domänen gegenseitig auf, so dass keine makroskopische

\footnotetext{
${ }^{(2)} \mathrm{Zu}$ Details zur Verbindung zwischen Magnetostriktion und kristallfeldinduzierter Anisotropie sei auf $[38-40]$ verwiesen.
} 
Verzerrung besteht. Erst wenn in einem externen Feld die magnetischen Domänen ausgerichtet werden und die magnetischen Momente in die gleiche Richtung orientiert sind, heben sich die mikroskopischen Verzerrungen nicht mehr auf, und es kommt zu einer makroskopischen Dehnung (siehe Abbildung 2.7). Diese ist jedoch im Allgemeinen sehr klein. So beträgt in reinem Eisen die gemittelte Magnetostriktionskonstante für polykristallines Material $\lambda_{s}=\frac{2}{5} \lambda_{100}+\frac{3}{5} \lambda_{111}=-3,6 \cdot 10^{-6}$ [41], während Eisen-PalladiumLegierungen mit einer Zusammensetzung nahe $\mathrm{Fe}_{70} \mathrm{Pd}_{30}$ Werte von $\lambda_{s}=5 \cdot 10^{-5}$ erreichen [1].

\subsection{Der Invar-Effekt}

Als Invar-Effekt bezeichnet man das Auftreten besonders kleiner oder sogar negativer thermischer Ausdehnung in einem gewissen Temperaturbereich. Dabei überlagern sich die normale thermische Ausdehnung, die auf anharmonische Therme im interatomaren Potential zurückgeht, und eine Kontraktion, die von einer Änderung des magnetischen Zustandes verursacht wird. Seinen Namen erhielt der Invar-Effekt nach der 1897 von Guillaume entdeckten Invar-Legierung $\mathrm{Fe}_{66} \mathrm{Ni}_{34}$ [42], deren effektiver thermischer Ausdehnungskoeffizient tatsächlich bei Zimmertemperatur vollständig verschwindet. Wird Nickel durch andere Elemente mit gleicher Elektronenkonfiguration wie z.B. Palladium oder Platin ersetzt, tritt ebenfalls anomale thermische Expansion auf. Der Invar-Effekt wurde inzwischen jedoch auch in vielen anderen Legierungen unterschiedlicher Zusammensetzung beobachtet [43]. Die genauen mikroskopischen Mechanismen, die zum Auftreten des Invar-Effektes führen, sind komplex. Allgemein wird eine Konkurrenz zweier magnetischer Zustände zugrunde gelegt: Weiss postulierte die Koexistenz eines ferromagnetischen Zustandes mit hohen magnetischen Momenten und großem Volumen neben einem antiferromagnetischen Zustand geringen Volumens und niedriger Momente [44]. Der Invar-Effekt beruht dabei auf einer thermischen Anregung vom ersteren in den letzteren Zustand, die die thermische Ausdehnung überlagert. Kakehashi et al. hingegen postulieren einen graduellen Übergang von starkem zu schwachem Magnetismus aufgrund thermischer Anregung [45]. Eine Übersicht wurde z.B. von Entel und Wassermann erstellt [46, 47]. Schilfgaarde et al. fanden schließlich eine umfassende Erklärung der Magneto-Volumen-Instabilität als Resultat nichtkollinearer Spinkonfiguration [48].

\subsection{Die Legierung Eisen-Palladium}

Dieser Abschnitt gibt einen Überblick über die bekannten Eigenschaften der Legierung Eisen-Palladium, beginnend bei strukturellen Daten und bekannten Untersuchungen zur Phasentransformation bis zu aktuellen Untersuchungen zur potentiellen Energielandschaft und deren Einfluss auf die mechanische Stabilität binärer Phasen.

Das Gleichgewichtsphasendiagramm der binären Legierung ist gut erforscht und in Abbildung 2.8 dargestellt [19]. Für diese Arbeit ist insbesondere der Kompositionsbereich um $\mathrm{Fe}_{70} \mathrm{Pd}_{30}$ mit einer Elektronenkonzentration von 8,6 Valenzelektronen pro Atom relevant. Bei hohen Temperaturen ist eine chemisch ungeordnete, kubisch flächenzentrierte (fcc für face centered cubic) Mischkristall-Phase, der Austenit, stabil. 


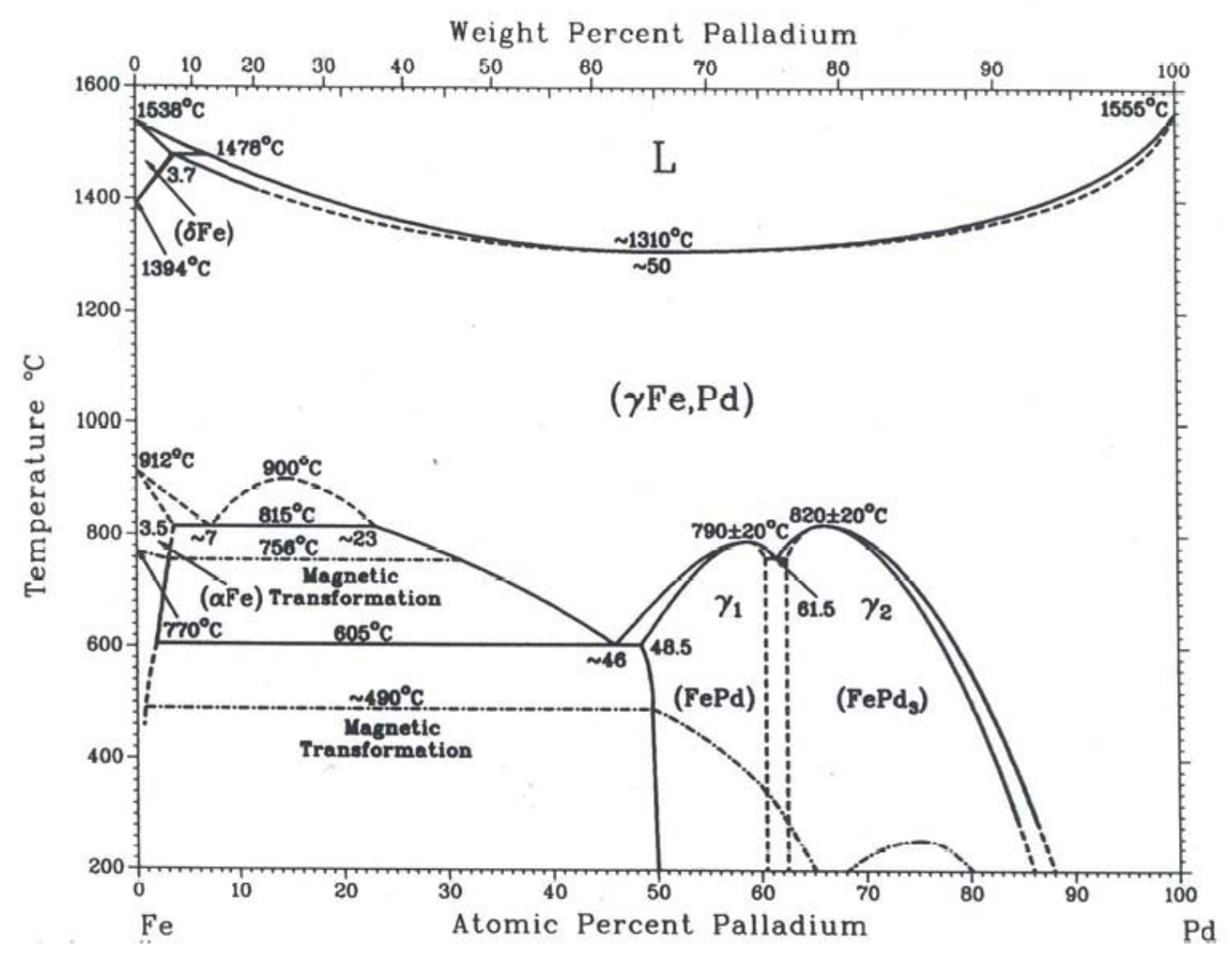

Abbildung 2.8: Gleichgewichtsphasendiagramm der Legierung Eisen-Palladium aus [19].

Bei Raumtemperatur sind die Gleichgewichtsphasen $\alpha$-Eisen und geordnetes $\mathrm{Fe}_{50} \mathrm{Pd}_{50}$ in der $\mathrm{L} 1_{0}$ Struktur. Allerdings ist das Entmischen der Legierung bei niedrigen Temperaturen kinetisch gehemmt, so dass durch Abschrecken von hohen Temperaturen der Mischkristall auch nahe Zimmertemperatur bewahrt werden kann. Neben dem kubisch flächenzentrierten Austenit können so auch eine tetragonal raumzentrierte (bct, body centered tetragonal) oder tetragonal flächenzentrierte (fct, face centered tetragonal) Martensitphase gewonnen werden [3, 6], wie in Abbildung 2.9 dargestellt ist. Matsui et al. bemerkten dabei, dass neben der Zusammensetzung der Legierung auch die Abkühlgeschwindigkeit die resultierende Phase beeinflusst [3]. Sie geben jedoch keine Begründung für die mikroskopischen Hintergründe dieser Abhängigkeit, welche auch die Unterschiede zu den Ergebnissen von Cui et al. erklären würde.

Der Übergang zur raumzentrierten Phase ist nicht thermoelastisch und kann deshalb nicht für den Formgedächtniseffekt genutzt werden [49]. Die Transformation zwischen fct-Martensit und fcc-Austenit hingegen ist reversibel. Liegt der Palladiumgehalt bei $29 \%$ bis $30 \%$, findet der martensitische Phasenübergang nahe Zimmertemperatur statt. So geben Cui und James an, dass in einem unbelasteten Einkristall der Zusammensetzung $\mathrm{Fe}_{70} \mathrm{Pd}_{30}$ die Umwandlung von Austenit zu Martensit zwischen $M_{s}=40^{\circ} \mathrm{C}$ und $M_{f}=12^{\circ} \mathrm{C}$ stattfindet [15]. Die Transformationstemperatur steigt mit dem Eisengehalt, der in binären Legierungen direkt mit der Valenzelektronenkonzentration 

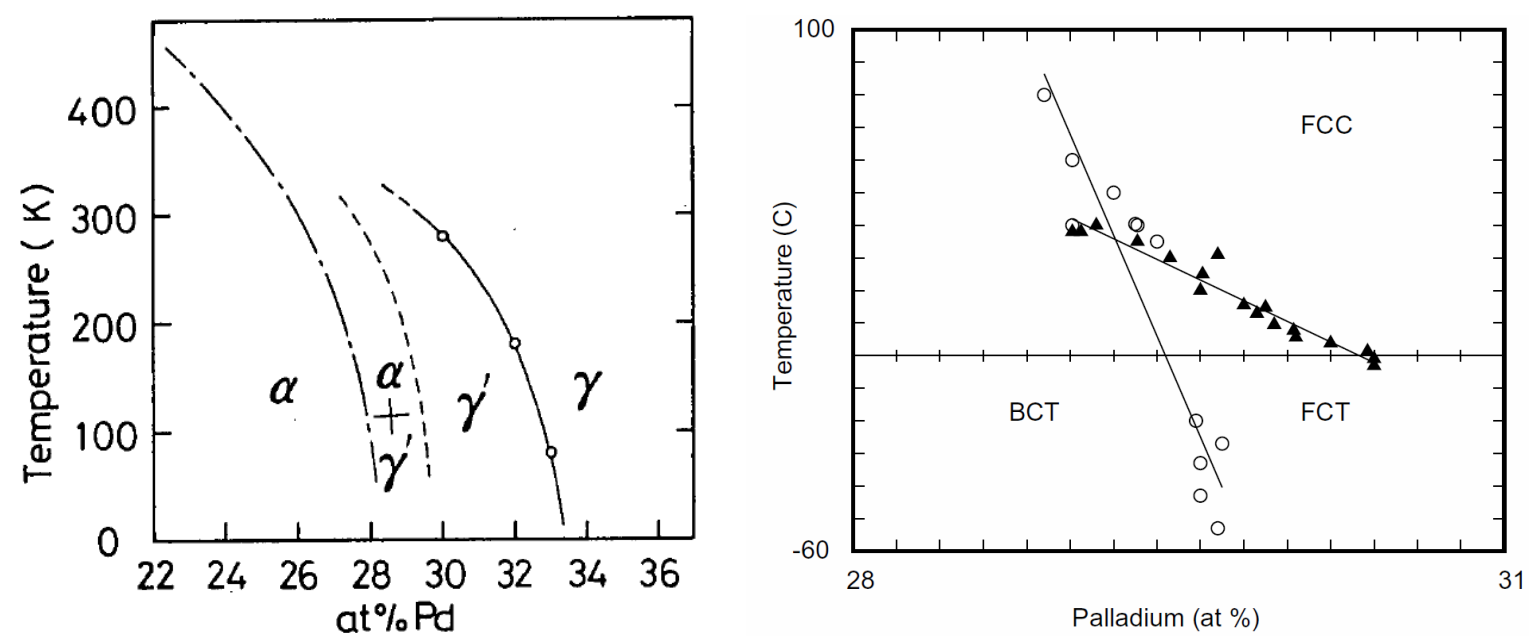

Abbildung 2.9: Metastabile Phasendiagramme der Legierung Eisen-Palladium nach Matsui [3] für Polykristalle (links) und Cui [6] für Einkristalle (rechts). Matsui et al. bezeichnen die bcc-Phase mit $\alpha$, die fcc-Phase mit $\gamma$ und die fct-Phase mit $\gamma^{\prime}$.

bzw. der Füllung des $d$-Bandes verbunden ist. Wie in Abschnitt 2.1 bereits erwähnt, beeinflusst auch mechanische Spannung die Transformation. So berichten Kato et al. von einer Änderung der Transformationstemperatur $\frac{\mathrm{d} \sigma}{\mathrm{dT}}=4,8 \mathrm{MPa} /{ }^{\circ} \mathrm{C}[50]$.

Die genauen Übergangstemperaturen hängen jedoch auch von weiteren experimentellen Parametern ab, so dass zum Teil deutliche Diskrepanzen zwischen den Ergebnissen verschiedener Gruppen auftreten. So fanden Shield und Cui Werte von $M_{s}=22,1^{\circ} \mathrm{C}$ und $M_{f}=16,5^{\circ} \mathrm{C}$ für einen Einkristall, der mit einem Druck von $2 \mathrm{MPa}$ belastet wurde [5]. Auch präparative und mikrostrukturelle Einflüsse prägen die Temperaturabhängigkeit dieses Überganges. Daher liegt die von Matsui et al. an $\mathrm{Fe}_{70} \mathrm{Pd}_{30}$-Polykristallen gefundene Übergangstemperatur von $7^{\circ} \mathrm{C}$ deutlich niedriger [3]. Des Weiteren fanden Sugimura et al. heraus, dass in dünnen Schichten der Phasenübergang zum Martensit bei höheren Temperaturen und geringerer Palladiumkonzentration stattfindet als im Massivmaterial [49].

Durch eine kontinuierliche Verzerrung entlang des sogenannten Bain-Pfades kann der kubisch flächenzentrierte (fcc) Austenit über den tetragonal flächenzentrierten (fct) Martensit und den tetragonal raumzentrierten (bct) Martensit in eine kubisch raumzentrierte (bcc) Phase überführt werden [52]. Nach Bain können alle diese Phasen als tetragonal raumzentrierte Strukturen mit verschiedenem Achsenverhältnis $a_{b c t} \mathrm{zu} c_{b c t}$ aufgefasst werden. Der Weg von fcc nach bcc kann dann gemäß Abbildung 2.10 als kontinuierliche tetragonale Verzerrung mit sinkendem $c / a$-Verhältnis beschrieben werden.

Die Austenitphase (fcc), im kubisch flächenzentrierten System mit einem Achsenverhältnis $c_{f c c} / a_{f c c}=1$, kann im raumzentrierten System durch $c_{b c t} / a_{b c t}=\sqrt{2}$ beschrieben werden. Dabei bleibt die $c$-Achse unverändert, also $c_{b c t}=c_{f c c}$, während die $a$-Achsen entlang der Flächendiagonalen der flächenzentrierten Struktur weisen und damit $a_{b c t}=1 / \sqrt{2} a_{f c c}$ ist. Im Zuge der Bain-Transformation wird die $c$-Achse kontinuierlich gestaucht, während die $a$-Achsen an Länge gewinnen, so dass das Volumen der Einheitszelle in erster Näherung konstant bleibt. Die fct-Phase und die bct-Phase 


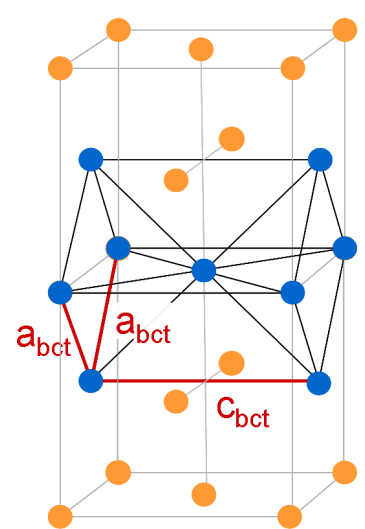

fCC

$\mathrm{c} / \mathrm{a}=1,41$

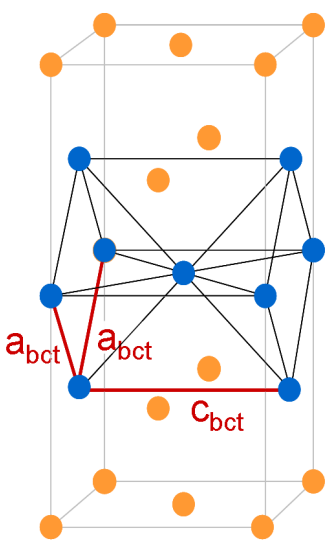

fct

$\mathrm{c} / \mathrm{a}=1,33$

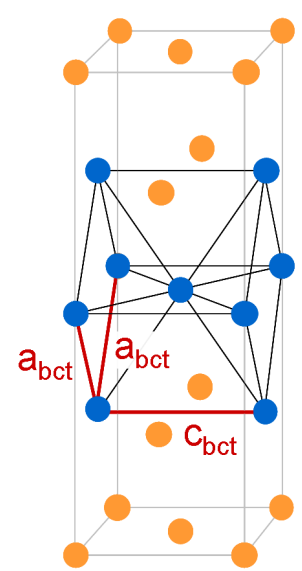

bct

$\mathrm{c} / \mathrm{a}=1,02$

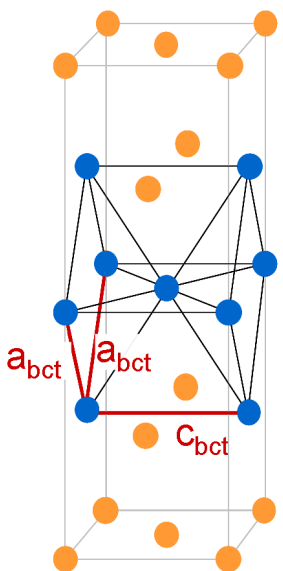

bcc

$\mathrm{c} / \mathrm{a}=1,00$

Abbildung 2.10: Aus der fcc-Phase entstehen durch tetragonale Verzerrung die fct-, bct- und bcc-Phase [51].

unterscheiden sich also nur im Grad der tetragonalen Verzerrung und damit im Achsenverhältnis $c / a_{b c t}$ von 1,33 (fct) bzw. 1,02 (bct). Zu beachten ist allerdings, dass neben der fcc- und der bcc-Phase bei tiefen Temperaturen nur diese beiden Werte des Achsenverhältnisses stabil sind. Bei höheren Temperaturen werden auch weitere Abstufungen zwischen der fcc- und der fct-Phase beobachtet. Obwohl die martensitische Transformation, wie in Abschnitt 2.1 erwähnt, in der Regel ein Phasenübergang erster Ordnung ist, fanden schon Matsui et al., dass die Änderung des Achsenverhältnisses in Eisen-Palladium-Legierungen nicht sprunghaft erfolgt [3]. Zwar steigt die tetragonale Verzerrung zu Beginn des Phasenüberganges sehr steil an, bei weiterem Kühlen nimmt sie jedoch bis zu sehr tiefen Temperaturen stetig weiter zu [3].

Als Ursache für den martensitischen Übergang von der fcc- in die fct-Struktur postulieren Opahle et al. einen Band-Jahn-Teller-Mechanismus, der aus der Entartung der elektronischen Zustände an der Fermikante resultiert [53]. Wird die Symmetrie des Kristalls durch eine tetragonale Verzerrung reduziert, wird die Entartung aufgehoben, was zu einem Energiegewinn von $14 \mathrm{meV} /$ Atom führt. Da die Energiedifferenzen zwischen den verschiedenen Strukturen äußerst gering sind, beeinflusst ein Energiegewinn dieser Größe die Freie Enthalpie signifikant. Berechnungen von Buschbeck et al. zeigen, dass die potentielle Energielandschaft entlang des Bain-Pfades weitestgehend flach ist [51]. In Abbildung 2.11 ist die Energie pro Atom für verschiedene Achsenverhältnisse aufgetragen. Die grauen Linien skizzieren den Verlauf des Potentials für die reinen Elemente Eisen bzw. Palladium. Die rote Kurve hingegen gibt die Energielandschaft für die Legierung $\mathrm{Fe}_{70} \mathrm{Pd}_{30}$ bei $0 \mathrm{~K}$ wieder. Neben einem globalen Minimum bei $c / a_{b c t}=1,33$ (fct) existiert ein lokales Minimum bei $c / a_{b c t}=1$ (bcc) sowie ein Plateau bei $c / a_{b c t}=1,41$ (fcc). Dies bedeutet, dass auch bei sehr tiefen Temperaturen die fct-Phase energetisch am günstigsten ist. Da die Energiedifferenz zur fcc-Struktur so klein ist, ist das Material gegenüber tetragonaler Verzerrung jedoch sehr instabil. Durch Einflüsse der Entropie bei endlichen Temperaturen können sich die Enthalpien der verschiedenen Phasen relativ zueinander leicht verschieben, so dass beispielsweise die Enthalpie der bcc-Struktur am kleinsten wird, was die Konsistenz mit den Messwerten von Cui et al. 


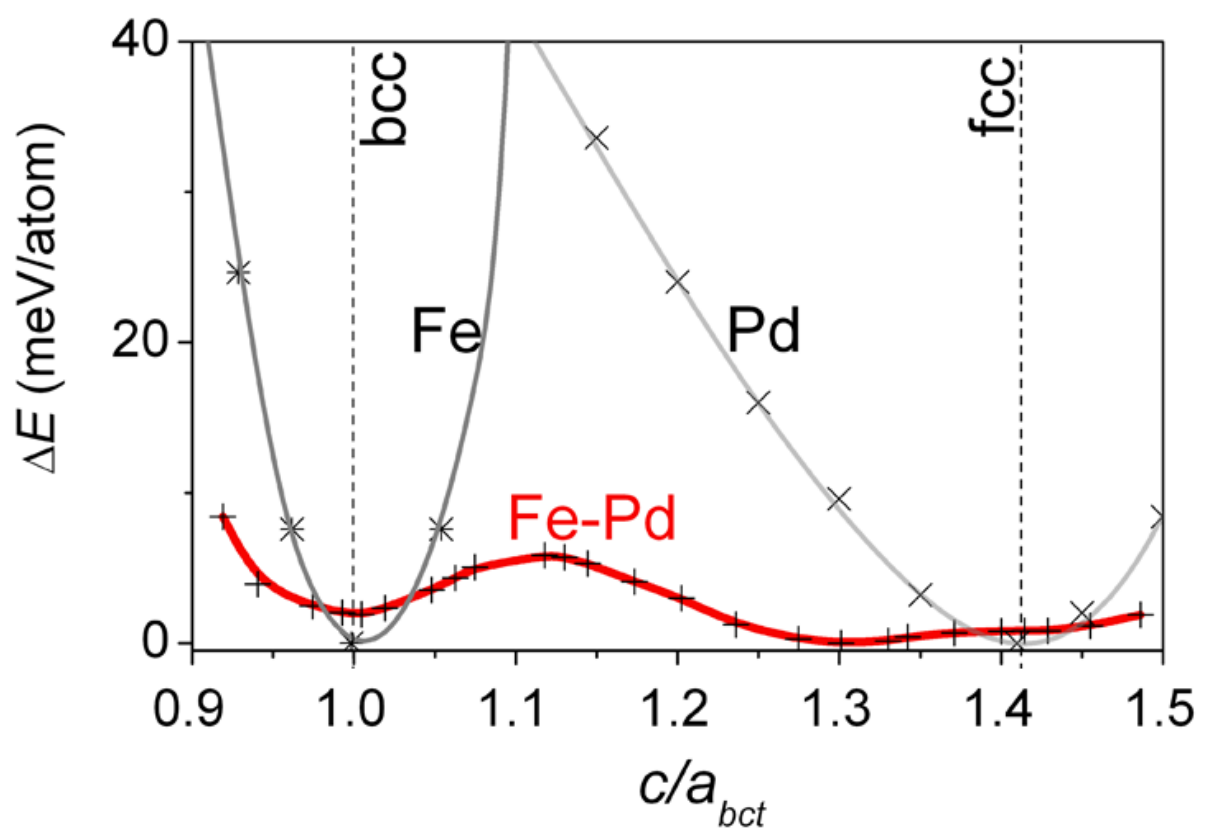

Abbildung 2.11: Die Energie pro Atom ändert sich bei tetragonaler Verzerrung entlang des Bain-Pfades kaum (aus [51]).

wiederherstellen würde [6]. Zudem wurde in den Berechnungen von Buschbeck et al. ein starres Gitter angenommen, ohne Relaxationsprozesse zu berücksichtigen. Neueste Berechnungen an relaxierten Strukturen deuten ein globales Minimum der bcc-Struktur an, insbesondere für (lokal) geordnete Strukturen [54].

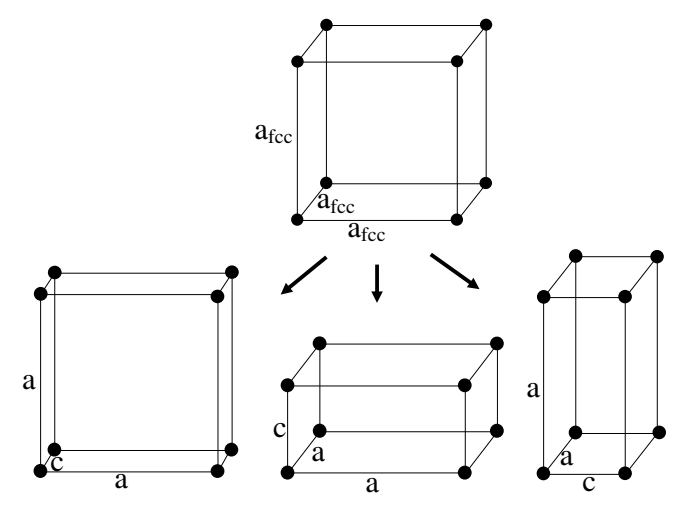

Abbildung 2.12: Aus der kubischen Phase entstehende Martensitvarianten [6].

Da in dieser Arbeit in erster Linie die fccund fct-Phase sowie der martensitische Übergang zwischen diesen beiden Phasen untersucht werden sollen, wird im Folgenden wieder die Notation des flächenzentrierten Systems aufgenommen. Die Gitterkonstante des kubisch flächenzentrierten Austenits in $\mathrm{Fe}_{70} \mathrm{Pd}_{30}$-Einkristallen bestimmten Cui und James zu $a_{f c c}=3,756 \AA$ [6]. Die Einheitszelle des tetragonal flächenzentrierten Martensits hat zwei gleich lange Basisvektoren mit dem Gitterparameter $a$ und einen etwas kürzeren der Länge $c$. Damit entstehen aus der kubischen Phase drei mögliche Martensitvarianten, die in Abbildung 2.12 dargestellt sind. Die genauen Werte der Gitterparameter $a$ und $c$ hängen signifikant von der Temperatur ab, da die tetragonale Verzerrung beim Kühlen weiter zunimmt. Cui et al. fanden $a=3,822 \AA$ und $c=3,630 \AA$ als über die Temperatur gemittelte Werte, was einem Achsenverhältnis von $c / a=0,950$ entspricht [6]. Die Extrapolation von Matsui et al. liefert hingegen bei $0 \mathrm{~K} c / a=0,914$ [3]. Damit kann durch vollständige Ausrichtung der Martensitvarianten eine maximale Längenänderung von $6 \%$ erreicht werden. 
Obwohl sowohl Austenit als auch Martensit ferromagnetisch sind, unterscheiden sich ihre magnetischen Eigenschaften deutlich, da sie eng mit der Kristallstruktur verknüpft sind. Im Austenit liegt die magnetische Vorzugsrichtung entlang der [111]-Richtung [14]. Die Sättigungsmagnetisierung bei $60^{\circ} \mathrm{C}$ wurde von Cui et al. zu $1080 \pm 10 \mathrm{emu} / \mathrm{cm}^{3}$ bestimmt [6]. Die Curie Temperatur liegt bei $450^{\circ} \mathrm{C}$, wobei die magnetische Suszeptibilität bereits bei $300^{\circ} \mathrm{C}$ stark abfällt.

Im Martensit liegt die magnetische Vorzugsrichtung parallel zu den langen $a$-Achsen der Einheitszelle, also in [001]- bzw. [010]-Richtung. Die magnetische Anisotropie ist wesentlich stärker als im Austenit und steigt mit fallender Temperatur. Die Sättigungsmagnetisierung wurde bei $-20^{\circ} \mathrm{C} z u 1200 \mathrm{emu} / \mathrm{cm}^{3}$ bestimmt [6], ist also ebenfalls geringfügig größer als im Austenit.

\subsection{Ternäre Systeme}

Aufgrund ihrer zahlreichen günstigen Eigenschaften wie hoher Duktilität, großer magnetokristalliner Anisotropie und hoher Sättigungsmagnetisierung sind binäre EisenPalladium-Legierungen vielversprechende Kandidaten für praktische Anwendungen, die sich beispielsweise den thermisch induzierten oder magnetischen Formgedächtniseffekt zunutze machen. Viele Anwendungen erfordern jedoch Zuverlässigkeit beim Einsatz bei Temperaturen oberhalb von Zimmertemperatur. Da in binären Eisen-PalladiumLegierungen die maximale magnetfeldinduzierte Dehnung erst bei sehr tiefen Temperaturen auftritt [55], ist eine Erhöhung der Übergangstemperatur wünschenswert. Zudem wäre eine erhöhte Stabilität gegenüber Entmischen bei mäßigen Abkühlraten für unkomplizierte Verarbeitung des Materials vorteilhaft. Das Zulegieren eines dritten Elements mit anderer Elektronenzahl oder anderem Atomradius erscheint hier erfolgversprechend.

In geordneten Eisenlegierungen verursachen die tetragonalen Verzerrungen eine deutliche Veränderung der eisendominierten Bereiche der Fermifläche. So kann die elektronische Instabilität, der die Verzerrung zugeschrieben wird, mit den lokalen Zuständen von Eisenatomclustern in einer bestimmten Umgebung verknüpft werden. Wie in Abschnitt 2.7 erwähnt, wird die martensitische Transformation durch den Eisengehalt beeinflusst. Allerdings scheint eine geeignete Valenzelektronenkonzentration nötig zu sein, um die elektronischen Zustände an der Fermikante zu halten. In binären Legierungen kann der Eisengehalt nicht verändert werden, ohne gleichzeitig die Valenzelektronenkonzentration zu verändern, so dass es schwierig ist, den Einfluss dieser beiden Faktoren zu unterscheiden. Ternäre Systeme hingegen bieten die Möglichkeit, beide Faktoren unabhängig voneinander zu variieren.

Wie in Abschnitt 2.7 beschrieben, unterliegt die Transformationstemperatur jedoch auch präparativen Einflüssen, weshalb die von verschiedenen Gruppen gefundenen Abhängigkeiten nicht immer konsistent sind. So berichten Tsuchiya et al., dass die Zugabe von Kobalt die Transformationstemperatur erhöht, während Nickel sie absenkt [56]. Kobalt- und nickelhaltige Legierungen verschiedener Zusammensetzung zeigen dabei eine gleichartige Abhängigkeit der Transformationstemperatur von der Valenzelektronenkonzentration, die jedoch nicht mit der des binären Systems übereinstimmt. Im Gegensatz dazu berichten sowohl Vokoun et al. als auch Sánchez-Alarcos et al., dass 
die Zugabe von Kobalt zwar die Bildung der bct-Phase verhindert, gleichzeitig aber die Transformationstemperatur senkt $[57,58]$. Zu beachten ist dabei allerdings, dass sich die Proben der beiden Gruppen sowohl im Palladiumgehalt als auch in der Präparation, insbesondere in der Kühlrate, unterscheiden. Die Substitution von Palladium durch isoelektronische Elemente wie Nickel oder Platin hingegen führt unbestritten zu einer Absenkung der Transformationstemperatur [57, 59]. Des Weiteren finden sich in der Literatur verschiedene Studien zum Einfluss weiterer Elemente wie Mangan, Rhodium oder Gallium auf das Transformationsverhalten [60-63].

In dieser Arbeit soll untersucht werden, inwiefern die Zugabe von Kupfer das Verhalten der Legierung beeinflusst. Dieses System ist besonders vielversprechend, da Kupfer durch seine inhärente fcc-Struktur als Austenitstabilisator wirken könnte [25].Außerdem hat Kupfer mehr Valenzelektronen als Eisen oder Palladium, so dass die Zugabe von Kupfer allgemein die Valenzelektronenkonzentration erhöht. Alternativ kann bei konstanter Valenzelektronenkonzentration der Eisengehalt auf Kosten des Palladiumgehaltes erhöht werden.

Bisher ist das System Eisen-Palladium-Kupfer wenig erforscht, und im Wesentlichen beschränken sich die Untersuchungen auf geordnetes Material mit Eisen- und Palladiumgehalt jeweils nahe 50\% [64, 65], in denen beispielsweise der Formgedächtniseffekt nicht zu erwarten ist. Neueste Arbeiten von Hamann et al. sind jedoch sehr vielversprechend [25]. Mit Hilfe von ab initio-Berechnungen getroffene Voraussagen werden hier durch eine systematische kombinatorische Untersuchung sputterdeponierter polykristalliner Schichten verschiedener Zusammensetzungen verifiziert. Generell zeigen die Daten einen Anstieg der Übergangstemperatur mit steigendem Eisengehalt bzw. sinkender Valenzelektronenkonzentration. Dabei ist jedoch zu beachten, dass in dünnen Schichten auf Substraten zum Teil intrinsische Verspannungen auftreten, die Übergangstemperatur, Gitterkonstanten, bei Zimmertemperatur auftretende Phasen und andere Eigenschaften beeinflussen können [51, 66, 67]. Daher sollen als Teil dieser Arbeit für ausgewählte Zusammensetzungen die Übertragbarkeit der Dünnschichtresultate auf das Massivmaterial überprüft und eventuelle Abweichungen untersucht werden. 


\section{Kapitel 3}

\section{Experimentelle Methoden}

In diesem Kapitel werden die experimentellen Methoden zur Herstellung und Charakterisierung der Proben vorgestellt. Insbesondere soll auf den Aufbau der Vibrating-ReedApparatur zur Untersuchung der mechanischen Eigenschaften eingegangen werden.

\subsection{Probenpräparation}

Die gewünschte austenitische bzw. martensitische Phase der Legierung Eisen-Palladium ist bei Zimmertemperatur metastabil. Soll die Austenitphase, die bei hohen Temperaturen Gleichgewichtsphase ist, nahe Zimmertemperatur bewahrt werden, müssen die Proben schnell abgekühlt werden, damit sie nicht in die stabilen Phasen $\alpha$-Fe und $\mathrm{Fe}_{50} \mathrm{Pd}_{50}$ entmischen. Um dünne Folien mit einer Dicke im Bereich mehrerer Mikrometer mit hohen Abkühlraten herzustellen, bietet sich daher die Technik des Splatquenching oder Ultra Rapid Quenching (URQ) an. Hier wird die geschmolzene Legierung zwischen zwei zusammenschlagenden Kupferstempeln abgeschreckt, so dass Abkühlraten von bis zu $10^{6} \mathrm{~K} / \mathrm{s}$ erreicht werden können [22, 23].

Zuvor muss eine binäre bzw. ternäre Vorlegierung hergestellt werden. Dazu werden die elementaren Materialien (siehe Tabelle 3.1) entsprechend der gewünschten Zusammensetzung eingewogen. Hierbei kann bei einer Materialmenge von $1-2 \mathrm{~g}$ eine Genauigkeit von etwa 0,01 Atomprozent erreicht werden. Die Oberflächen der eingewogenen Materialien werden mit Aceton und Isopropanol im Ultraschallbad gereinigt.

\begin{tabular}{c|c|c|c} 
Material & Hersteller & Form & Reinheit \\
\hline Eisen & KURT J. LESKER & Pellets & $99,95 \%$ \\
Palladium & HERAEUS & Granalien & $99,95 \%$ \\
Kupfer & MATEK & Kügelchen & $99,99 \%$
\end{tabular}

Tabelle 3.1: Hersteller und Reinheit der verwendeten Materialien

In einem Lichtbogenofen, dem sogenannten Arcmelter werden die Materialien miteinander legiert. Dabei liegen die Materialstücke auf einer wassergekühlten Kupferplatte in einer Vakuumkammer, die zunächst auf einen Druck von ca. $3 \cdot 10^{-3}$ mbar abgepumpt 
und mit Argon gespült wird. Dieser Vorgang wird sechsmal wiederholt. Anschließend wird die Kammer bis zu einem Arbeitsdruck von 600 mbar mit Argon der Reinheit 5.0 befüllt. Um den Restsauerstoffgehalt in der Kammer zu reduzieren, wird dann ein chemisch sehr reaktiver Zirkonblock mehrfach aufgeschmolzen. Nun werden die eingewogenen Materialien miteinander verschmolzen. Um die Proben zu homogenisieren, wird die entstandene Legierungskugel mehrmals nach dem Erstarren gewendet und wieder aufgeschmolzen. Eventuell vorhandene oxidische Verunreinigungen scheiden sich bevorzugt an der Oberfläche ab und können nach dem Ausbau aus der Kammer durch Sandstrahlen entfernt werden. Nun wird die Kugel in kleine Stücke zerteilt, die wiederum in Portionen zu je $180 \mathrm{mg}$ bis $200 \mathrm{mg}$ eingewogen und im Arcmelter zu Kügelchen geschmolzen werden.

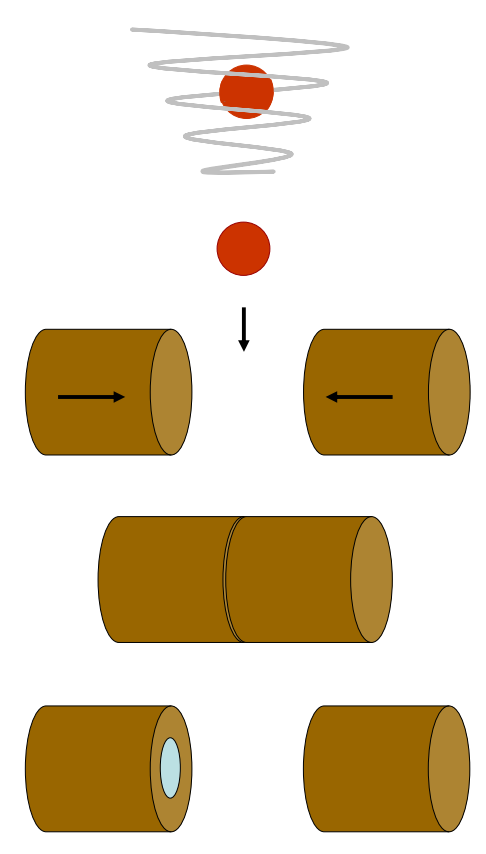

Abbildung 3.1: Beim Splatquenching wird das Material induktiv aufgeschmolzen und zwischen zwei Kupferstempeln abgeschreckt.
Die so vorlegierten Kügelchen werden einzeln in einen URQ-Apparat der EDMUND BÜHLER GMBH eingebaut, der wiederum sechsmal abgepumpt und gespült und bis zu einem Arbeitsdruck von 600 mbar mit Argon der Reinheit 5.0 befüllt wird. Das Legierungskügelchen wird auf einem Keramikprobenhalter in der Mitte einer speziell geformten Spule positioniert (siehe Abbildung 3.1). Diese wird von einem hochfrequenten Wechselstrom durchflossen, der zum einen die Probe induktiv aufheizt und sie zum anderen durch Induktion von Kreisströmen zum Schweben bringt. Sobald das Material vollständig aufgeschmolzen ist, wird der Strom abgestellt, so dass die Probe frei nach unten fällt. Hierbei passiert sie eine Lichtschranke, die den Auslösemechanismus zweier Kupferstempel aktiviert. Diese schlagen zusammen und pressen das Legierungskügelchen zu einer ca. $50 \mu \mathrm{m}$ dicken Folie, dem sogenannten Splat.

Wegen der extrem hohen Erstarrungsgeschwindigkeit bei Abkühlraten von bis zu $10^{6} \mathrm{~K} / \mathrm{s}$ weisen die Splats eine sehr hohe Defektdichte und große innere Verspannungen auf, die den martensitischen

Phasenübergang oder den magnetischen Formgedächtniseffekt behindern können [24]. Deshalb wurden einige Proben einer Glühbehandlung unterzogen, so dass Defekte ausheilen und innere Verspannungen abgebaut werden können. Um Oxidation während der Glühbehandlung zu vermeiden, wurden die Proben unter 600 mbar Argonschutzatmosphäre (Reinheit 5.0) in Quarzröhrchen eingeschweißt. Diese wurden bei Temperaturen zwischen $600^{\circ} \mathrm{C}$ und $1000^{\circ} \mathrm{C}$ in einen senkrechten Röhrenofen gehängt. Je nach gewünschter Abkühlgeschwindigkeit verblieben die Quarzröhrchen mit den Proben nach Ablauf der jeweiligen Glühzeit bis zum vollständigen Erkalten im Ofen, wurden aus dem Ofen genommen und an Luft abgekühlt, oder die Aufhängung wurde zerschnitten, so dass die Röhrchen in einen Wassereimer fielen und die Proben schnell abgeschreckt wurde. Kühlen die Proben im Ofen ab, so fällt (bei einer Ausgangstemperatur von $850^{\circ} \mathrm{C}$ ) ihre Temperatur näherungsweise exponentiell mit der Zeit ab und liegt erst 
nach etwa 30 Minuten unter $500^{\circ} \mathrm{C}$.

Neben der thermodynamischen Stabilität gegenüber Entmischen soll in dieser Arbeit auch die Stabilität der Kristallstruktur gegenüber mechanischer Verformung untersucht werden. $\mathrm{Zu}$ diesem Zweck wurden einige Proben mit einer Kaltwalze verformt. Um die Verformung verschiedener Proben vergleichen zu können, wurden die Proben auf die Hälfte ihrer ursprünglichen Dicke gewalzt. Um die Andruckkraft der Walzen zu erhöhen, eine gleichmäßige Verformung sicherzustellen und die Proben vor Verunreinigungen zu schützen, wurden sie im Sandwichverfahren zwischen zwei $1 \mathrm{~mm}$ starken Edelstahlblechen gewalzt. Um die Dicke der Proben auf die Hälfte zu reduzieren, waren sechs Walzgänge nötig. Andere Proben wurden durch manuelles Hämmern mechanisch verformt. Im Gegensatz zum Walzen induziert dieser Vorgang keine Vorzugsrichtung. Er ist jedoch nicht so präzise zu kontrollieren und wurde deshalb lediglich als Vergleich zum Kaltwalzen herangezogen.

\subsection{Charakterisierungsmethoden}

Einen wichtigen Teil dieser Arbeit bildet die strukturelle, magnetische und mechanische Charakterisierung der binären und ternären Eisen-Palladium-Folien. Die dabei verwendeten Methoden sollen in den folgenden Abschnitten kurz beschrieben werden.

\subsubsection{Strukturbestimmung}

Die Kristallstruktur polykristalliner Proben kann mittels Röntgendiffraktometrie in Bragg-Brentano-Geometrie bestimmt werden. Dabei werden Quelle, Probe und Detektor so ausgerichtet, dass der Winkel zwischen Primär- und Sekundärstrahl gerade doppelt so groß ist wie der Winkel $\theta$ zwischen Primärstrahl und Probenoberfläche. Die Röntgenstrahlung fällt unter dem Winkel $\theta$ auf die Probe, dringt in das Material ein und wird an den Atomen des Kristallgitters gestreut. Sofern die Änderung des Wellenvektors beim Streuprozess ein Vektor des reziproken Gitters ist, interferieren die reflektierten Strahlen von allen Gitterpunkten konstruktiv, und es kommt zu einem Maximum in der Intensität der gestreuten Strahlung. Eine Herleitung der Interferenzbedingung nach Laue findet sich in [68]. Dort wird auch gezeigt, dass sie äquivalent ist zur Bragg-Bedingung

$$
n \lambda=2 d_{h k l} \sin \theta,
$$

bei der $d_{h k l}$ der Abstand der Netzebenen des Kristallgitters im Realraum, $\lambda$ die Wellenlänge der Röntgenstrahlung und $n$ die Ordnung des Reflexes ist. So können aus den Positionen der Peaks Rückschlüsse auf die Netzebenenabstände und damit auf die Kristallstruktur der Schicht gezogen werden. Für die Messungen wurden ein Diffraktometer vom Typ D5000 der Firma Siemens und ein Diffraktometer vom Typ D8 der Firma BRUKER verwendet. Beide arbeiten mit der charakteristischen $\mathrm{K}_{\alpha}$-Strahlung einer Kupferanode, die eine mittlere Wellenlänge von 0,154 nm hat [69]. Die Röntgenmessungen zur Bestimmung der Kristallstruktur werden für verschiedene Temperaturen oberhalb und unterhalb der Umwandlungstemperatur durchgeführt. Dafür wurde ein Probenhalter angefertigt, der mit einem Peltierelement sowohl bis $100{ }^{\circ} \mathrm{C}$ geheizt als 
auch bis $5^{\circ} \mathrm{C}$ gekühlt werden kann. Um einen weiteren Temperaturbereich abzudecken, wurden außerdem Röntgenmessungen zwischen $-145^{\circ} \mathrm{C}$ und $40{ }^{\circ} \mathrm{C}$ von $\mathrm{S}$. Hamann am Institut für Werkstoffe der Ruhr-Universität Bochum durchgeführt. Bei Temperaturen unter $20^{\circ} \mathrm{C}$ werden die Proben mit einer evakuierten Beryllium-Haube vor Kondenswasser und Eisbildung geschützt. Um deren Einfluss auf das Röntgenspektrum zuverlässig zu identifizieren, wurde bei $20^{\circ} \mathrm{C}$ jeweils eine Messung mit und ohne Beryllium-Haube durchgeführt. Zur Untersuchung der Kristallstruktur unter mechnischer Belastung wurde ein weiterer Probenhalter angefertigt. Die Probe wird an beiden Enden zwischen Klemmbacken befestigt, die mittels einer Feingewindeschraube auseinandergezogen werden können, so dass die Probe uniaxial gedehnt wird.

Auch in polykristallinen Proben müssen nicht immer alle Kristallorientierungen gleichmäßig vertreten sein. Treten eine oder mehrere Orientierungen bevorzugt auf, so ist die Probe texturiert. Zur Bestimmung der Textur wird die Probe unter verschiedenen Winkeln geröntgt. Aus der Abhängigkeit der Streuintensität von der Probenausrichtung kann auf die Textur geschlossen werden. Zunächst wird in einer Weitwinkel-Messung die Position eines großen Reflexes von einer Ebenenschar mit dem Abstand $d_{h k l}$ bestimmt. Einfallswinkel $\theta$ und Reflexionswinkel $2 \theta$ werden so eingestellt, dass die BraggBedingung erfüllt ist. Im Folgenden wird diese Einstellung nicht mehr verändert. Damit die Probe aus verschiedenen Richtungen bestrahlt werden kann, wird sie um zwei zueinander senkrechte Achsen gedreht, und zwar um den Azimut $\phi$ um eine Achse senkrecht zur Probenoberfläche und um den Kippwinkel $\psi$ um eine Achse parallel zur Probenoberfläche und zur Einfallsebene, wie in Abbildung 3.2 dargestellt. Während der schrittweisen Drehung der Probe wird die Intensität der reflektierten Strahlung in $\theta / 2 \theta$-Geometrie gemessen und anschließend in einer stereographischen Projektion als zweidimensionale Polfigur in Abhängigkeit beider Winkel aufgetragen [70]. So kann die räumliche Ausrichtung der Ebenen $(h k l)$, die den Reflex verursachen, festgestellt werden. Die Texturmessungen wurden an einem Vierkreis-Röntgendiffraktometer vom Typ X'Pert der Firma PHILIPS mit einer Kobaltanode $(\lambda=0,179 \mathrm{~nm})$ vorgenommen [69].

Neben der Kristallstruktur und Textur wurde auch die Mikrostruktur der Proben unter-

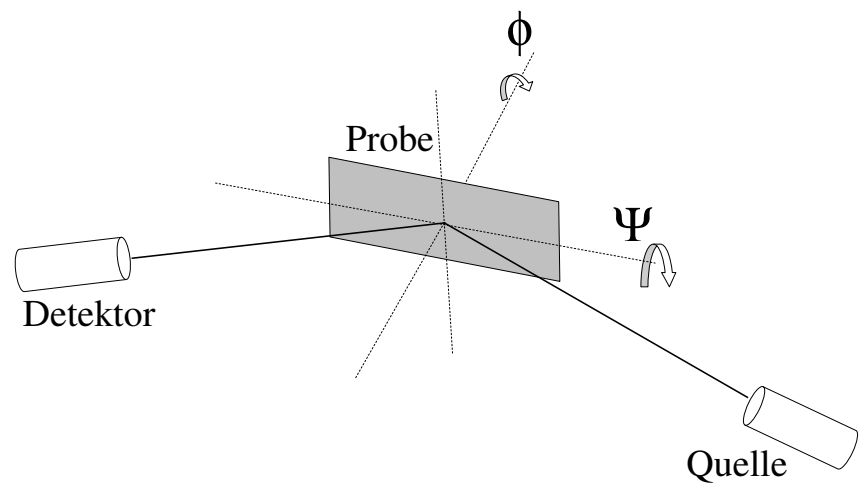
Abbildung 3.2: Bei der Texturmessung wird die Probe um den Azimut $\phi$ gedreht und
um den Kippwinkel $\psi$ gekippt. 
sucht. Dazu kamen ein Rasterelektronenmikroskop (REM) vom Typ LEO Supra 35 der Firma ZEISS und ein JEOL JSM 5800LV zum Einsatz. Ein fokussierter Elektronenstrahl rastert über die Probe, wodurch Sekundärelektronen aus dem Material ausgelöst werden. Ihre Intensität gibt Auskunft über die Morphologie der Oberfläche [27]. Je kleiner der Winkel zwischen Oberflächennormale und einfallendem Primärelektronenstrahl ist, desto mehr Sekundärelektronen werden emittiert, das heift umso heller erscheint die Oberfläche. Da die Sekundärelektronen hauptsächlich aus oberflächennahen Schichten emittiert werden, erscheinen herausragende Kanten zwischen zwei geneigten Flächen besonders hell. Eingeschnittene Gräben erscheinen entsprechend dunkel, da die Sekundärelektronen nur in einem sehr kleinen Winkelbereich emittiert werden.Allerdings ist der Kontrast an Oberflächenstrukturen der in dieser Arbeit untersuchten Proben teilweise recht gering, weshalb zusätzliche Untersuchungen mit optischer Mikroskopie und Transmissionselektronenmikroskopie (TEM) durchgeführt wurden, bei denen bessere Ergebnisse erzielt wurden. Für die TEM-Untersuchungen wurde zunächst mit einem fokussierten Ionenstrahl (Focused-Ion-Beam, FIB) ein Graben in die Probe hineingearbeitet, an dem mit dem REM erste Einblicke in die Mikrostruktur gewonnen werden konnten. Anschließend wurde eine Lamelle für den Einbau in das TEM herausgetrennt. Die Arbeiten mit dem REM, FIB und TEM wurden von S. Hamann, H. Brunken und D. König am Institut für Werkstoffe der Ruhr-Universität Bochum durchgeführt.

Zusammensetzung und Homogenität der Proben wurde mit Hilfe der energiedispersiven Röntgenanalyse (Energy Dispersive X-Ray Analysis, EDX) überprüft. Dabei werden anhand der vom Elektronenstrahl ausgelösten charakteristischen Röntgenstrahlung die in der Probe vorhandenen Elemente und deren Konzentrationen bestimmt. Neben Messungen am LEO Supra in Göttingen wurden einige Proben im Rahmen einer Kooperation des DFG Schwerpunktprogrammes SPP $1239{ }^{(1)}$ von S. Hamann mit einem JEOL JSM 5800LV am Institut für Werkstoffe der Ruhr-Universität Bochum charakterisiert, wo ein $\mathrm{Fe}_{70} \mathrm{Pd}_{30}$-Standard zur Kalibration des EDX zur Verfügung stand [24].

\subsubsection{Messung der Magnetisierung}

Die magnetischen Momente der Proben wurden in einem Superconducting Quantum Interference Device (SQUID) der Firma Quantum-Design gemessen. Dabei wird die Probe durch eine sogenannte Second Derivative Coil aus supraleitendem Material bewegt. Wie in Abbildung 3.3 dargestellt, sind die obere und die untere Windung dieser Spule in die gleiche Richtung und die mittleren beiden Windungen in die entgegengesetzte Richtung gewickelt. Diese Anordnung verbessert das Signal-zu-

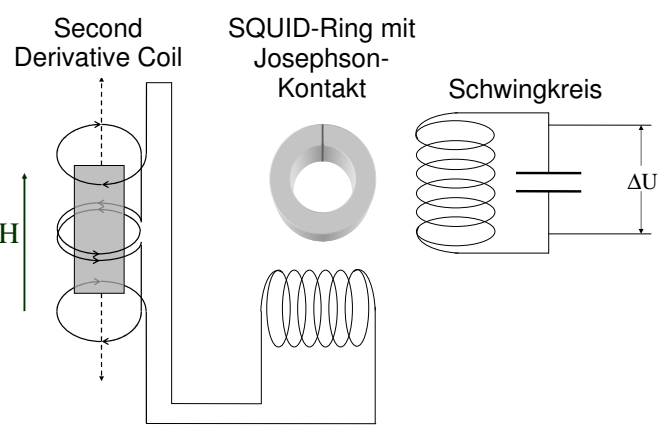

Abbildung 3.3: Das SQUID-System. Rauschen-Verhältnis. Eine periodische Bewegung der Probe durch die Second Derivative Coil induziert eine Spannung, die über eine Anordnung von supraleitenden Spulen induktiv an das SQUID gekoppelt ist. Das SQUID besteht aus einem supraleiten-

\footnotetext{
${ }^{(1)}$ Untersuchung der Änderung von Mikrostruktur und Form fester Werkstoffe durch äußere Magnetfelder
} 
den Ring mit einem Josephson-Kontakt, d. h. einem Supraleiter-Isolator-SupraleiterKontakt, in dem der Isolator so dünn ist, dass Cooper-Paare hindurchtunneln können [71].

Der SQUID-Ring befindet sich in der Spule eines Schwingkreises, der einen veränderlichen magnetischen Fluss und einen Wechselstrom im Ring induziert. Überschreiten der vom Schwingkreis und der von der Second Derivative Coil verursachte Fluss und damit der im SQUID-Ring induzierte Strom einen bestimmten Wert $I_{c}$, so wird beim Tunneln einzelner Elektronen am Josephson-Kontakt Energie dissipiert. Diese Energie geht dem Schwingkreis verloren, was als Spannungsabfall messbar ist. Über diesen Spannungsabfall wird mit Hilfe der Software des SQUID-Systems das magnetische Moment der Probe berechnet.

\subsubsection{Widerstandsmessung}

Der elektrische Widerstand einer Metallprobe kann als Indikator für strukturelle Veränderungen dienen. Für Widerstandsmessungen stand eine vollautomatisierte Messanlage zur Verfügung [72], in der eine Serie von Proben in einem Durchgang vermessen werden kann. Um Oxidation und Eisbildung zu vermeiden, wurden die Messungen unter Stickstoffatmoshäre durchgeführt. Ein Vierpunkt-Messkopf mit federgelagerten Kontakten wird nacheinander auf die Proben abgesenkt, um deren Widerstand bei einem konstanten Strom von $100 \mathrm{~mA}$ zu messen. Anschließend wird die Temperatur des Probentisches und damit der Proben geändert und es werden erneut die Widerstände bestimmt. Auf diese Weise wurden Messpunkte zwischen $-40^{\circ} \mathrm{C}$ und $140^{\circ} \mathrm{C}$ aufgenommen. Der Temperaturzyklus wurde mehrfach durchlaufen, um die Messgenauigkeit zu erhöhen und die Reversibilität zu prüfen. Die Widerstandsmessungen führte S. Hamann vom Institut für Werkstoffe der Ruhr-Universität Bochum im Rahmen einer Kooperation des DFG Schwerpunktprogrammes SPP1239 durch [24].

\subsubsection{Untersuchung der mechanischen Eigenschaften}

Wie in Kapitel 2 erwähnt, werden die besonderen Eigenschaften (wie z. B. der InvarEffekt, Superelastizität oder der Formgedächtniseffekt) der Legierung Eisen-Palladium durch das extreme Aufweichen des Kristallgitters in der Nähe des martensitischen Phasenüberganges ermöglicht. Daher ist die Untersuchung der mechanischen Eigenschaften von großem Interesse. Zum einen wurde in quasistatischen Zugversuchen das elastische Verhalten der Proben bei mittleren Dehnungen charakterisiert. Zum anderen wurden in einem neu aufgebauten Vibrating-Reed-Experiment Elastizität und Dämpfung für kleine Auslenkungen analysiert.

Die Zugversuche wurden im Rahmen einer Kooperation mit dem Lehrstuhl für anorganische Funktionsmaterialien an der Christian-Albrechts-Universität in Kiel, die ebenfalls durch das DFG Schwerpunktprogrammes SPP1239 gefördert wurde, von C. Bechtold an einer kommerziellen Z0.5 (Zwicki Line)-Anlage der ZwiCK \& RoELL GMBH durchgeführt. Ein schmaler Probenstreifen wird an beiden Seiten fest eingespannt und mit Hilfe eines elektromechanischen Antriebs schrittweise gedehnt, wobei die dazu notwendige Kraft protokolliert wird. Anschließend wird die Probe schrittweise wieder entlastet. Auch hierbei wird die Kraft aufgezeichnet. So ist es möglich, 
den elastischen Anteil der Längenänderung vom plastischen Anteil zu unterscheiden. In mehreren Durchläufen wird die Probe immer stärker gedehnt, so dass Informationen über die elastischen Eigenschaften bei verschiedenen Dehnungen gewonnen werden können. Die Längenänderung, die von der Dehnungsmesszelle ausgegeben wird, kann mit Hilfe eines Videoextensometers verifiziert werden. Anhand der mit einer Mikrometeruhr bestimmten Dicke sowie der Breite und Länge der Probe können aus der Längenänderung und der benötigten Kraft Dehnung und Spannung gewonnen werden.

Mit demselben Gerät kann auch die Längenänderung einer Probe in einem Magnetfeld gemessen werden. Da das Gestänge der Dehnungsmesszelle aus nichtmagnetischem Material gefertigt ist, kann einfach ein Magnetfeld von bis zu 0,5 T senkrecht zur Messrichtung angelegt und dabei die Längenänderung der Probe protokolliert werden.

Der Zugversuch ist so konzipiert, dass bei konstanter Temperatur die Spannung der Probe verändert wird. Um die Vorgänge während des Phasenüberganges zu beobachten, ist es sinnvoll, bei anderweitig konstanten Parametern die Temperatur zu variieren. Dies ist bei den oben genannten Anlagen jedoch nur eingeschränkt möglich. Um die Veränderung der elastischen Eigenschaften mit der Temperatur genauer zu untersuchen, wurde daher eine Vibrating-Reed-Apparatur entworfen und aufgebaut, die im folgenden Kapitel genau beschrieben wird.

\subsection{Das Vibrating Reed}

Mit einem sogenannten Vibrating-Reed-Experiment können die mechanischen Eigenschaften einer Probe untersucht werden [73]. Kernstück des Aufbaus ist ein schmaler länglicher Probenstreifen, der an einer Seite fest eingespannt ist und zu resonanter Schwingung angeregt wird, die mit Hilfe eines Lichtzeigers detektiert wird. Die abklingende freie Schwingung liefert Informationen über den Elastizitätsmodul und die Dämpfungseigenschaften der Probe. Alternativ können diese Informationen auch aus der Resonanzfrequenz und der Breite der Resonanzkurve sowie aus der Phasenverschiebung zwischen Anregung und Schwingung gewonnen werden [73]. Im Rahmen dieser Arbeit wurde im Wesentlichen die erste Möglichkeit genutzt. Aufbau und Funktionsweise der Vibrating-Reed-Apparatur sind in Abbildung 3.4 schematisch dargestellt und sollen in diesem Kapitel genauer beschrieben werden.

Zunächst soll jedoch auf die Theorie der Biegeschwingung eingegangen werden, um den Zusammenhang von Resonanzfrequenz, Eigenfrequenz, Amplitude, Elastizitätsmodul und Dämpfung zu verdeutlichen. Anschließend werden der Aufbau der VibratingReed-Apparatur sowie der Ablauf des Messverfahrens und der Auswertung detailliert beschrieben.

\section{Beschreibung der Schwingung}

Wenn die Probe aus der Ruhelage $z=0$ ausgelenkt wird und keine weiteren Kräfte wirken, führt sie in erster Näherung eine harmonische gedämpfte Biegeschwingung in der Grundmode durch. Im Folgenden sollen Form und Frequenzen einer solchen Schwingung hergeleitet werden (siehe dazu auch [74-76]). Dazu betrachten wir einen 


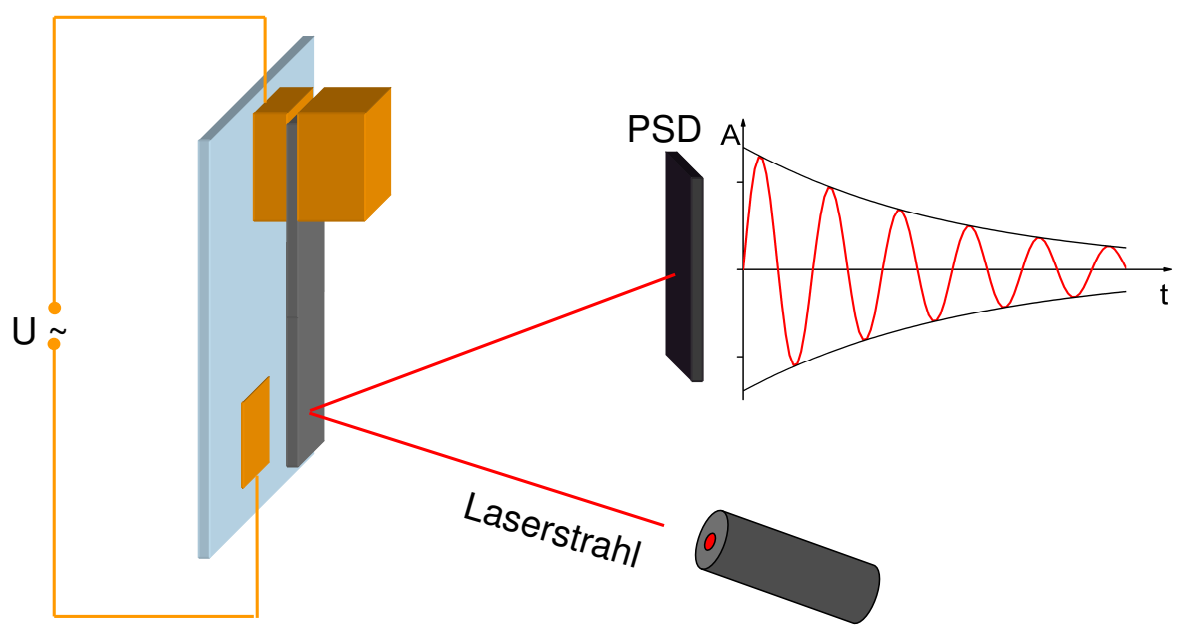

Abbildung 3.4: Das Vibrating Reed wird kapazitiv zu Schwingungen angeregt, die mit Hilfe eines Lichtzeigers detektiert werden.

homogenen elastischen Balken der Länge $L$, Breite $b$ und Dicke $d$ mit dem Elastizitätsmodul $E$, der wie in Abbildung 3.5 an der Stelle $x=0$ fest eingespannt ist. Wirkt auf den Balken eine äußere Kraft $F$, so biegt er sich so weit, bis die äußere Kraft durch die elastische Rückstellkraft $F_{R}$ kompensiert wird. Diese elastische Kraft entsteht, weil das Balkenmaterial oberhalb einer neutralen Faser gedehnt und unterhalb zusammengestaucht wird. Die dabei auftretenden Dehnungen $\epsilon=\Delta L / L$ sind in Abbildung 3.5 skizziert. Eine beliebige gedehnte Faser im Abstand $z$ von der neutralen Faser übt also die Spannung

$$
\sigma=E \cdot \epsilon=E \cdot \frac{z}{R}
$$

aus. Auf den gesamten Querschnitt des Balkens wirkt damit ein rückstellendes Drehmoment

$$
M_{R}=\int_{d / 2}^{-d / 2} \sigma(z) b z \mathrm{~d} z=\frac{b E}{R} \int_{d / 2}^{-d / 2} z^{2} \mathrm{~d} z=\frac{E b d^{3}}{12 R}
$$

Für kleine Auslenkungen, d.h. große Krümmungsradien gilt näherungsweise $\frac{1}{R}=\frac{d^{2} z}{d x^{2}}$ und damit

$$
M_{R}=\underbrace{\frac{E b d^{3}}{12}}_{:=c} \frac{\mathrm{d}^{2} z}{\mathrm{dx}^{2}}
$$

Ein Balken, der aus der Ruhelage ausgelenkt und dann losgelassen wird, führt wegen dieses rückstellenden Momentes eine Biegeschwingung aus, und zwar so, dass sich Trägheitskraft $F_{T}$, Reibungskraft $F_{D}$ und Rückstellkraft $F_{R}$ zu jeder Zeit an jedem Punkt des Balkens ausgleichen. Da diese Kräfte nicht über die gesamte Länge des Balkens konstant sind, müssen beim Aufstellen der Bewegungsgleichung die jeweiligen Kräfte auf ein infinitesimales Längenstück bezogen werden:

$$
\frac{\mathrm{d} F_{R}}{\mathrm{dx}}-\frac{\mathrm{d} F_{T}}{\mathrm{dx}}-\frac{\mathrm{d} F_{D}}{\mathrm{dx}}=0
$$



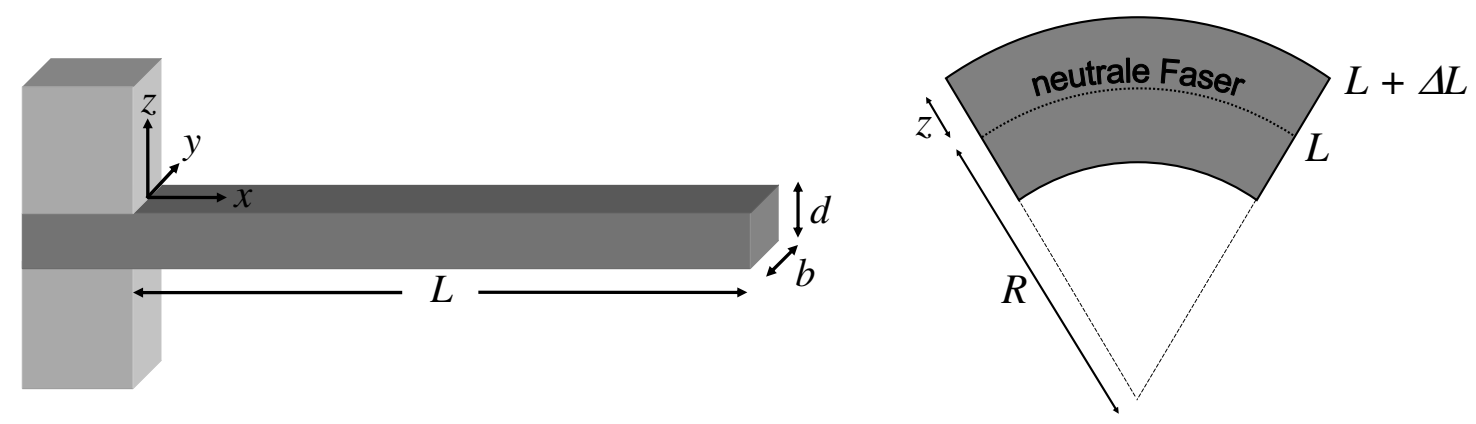

Abbildung 3.5: Wird der Balken gebogen, dehnt sich das Material oberhalb der neutralen Faser, so dass eine rücktreibende Kraft entsteht.

Die Rückstellkraft berechnet sich aus dem rückstellenden Moment $M_{R}=c \frac{\mathrm{d}^{2} z}{\mathrm{dx}^{2}} \mathrm{zu}$

$$
\frac{\mathrm{d} F_{R}}{\mathrm{dx}}=\frac{\mathrm{d}}{\mathrm{dx}} \frac{\mathrm{d} M_{R}}{\mathrm{dx}}=c \cdot \frac{\mathrm{d}^{4} z}{\mathrm{dx}^{4}}
$$

Die Trägheitskraft $F_{T}$ ergibt sich mit der Dichte $\rho$ zu

$$
\frac{\mathrm{d} F_{T}}{\mathrm{dx}}=\underbrace{\rho \cdot b \cdot d}_{:=\mu} \frac{\mathrm{d}^{2} z}{\mathrm{dt}^{2}},
$$

wobei $\mu$ die Masse pro Länge darstellt. Die Reibungskraft ergibt sich mit einem geeigneten Reibungskoeffizienten pro Länge $D$ zu

$$
\frac{\mathrm{d} F_{D}}{\mathrm{dx}}=D \frac{\mathrm{d} z}{\mathrm{dt}}
$$

Damit erhalten wir die Bewegungsgleichung

$$
c \cdot \frac{\mathrm{d}^{4} z}{\mathrm{dx}^{4}}-\mu \cdot \frac{\mathrm{d}^{2} z}{\mathrm{dt}^{2}}-D \cdot \frac{\mathrm{d} z}{\mathrm{dt}}=0
$$

die sich durch Separation in einen zeitabhängigen Teil $g(t)$ und einen ortsabhängigen Teil $h(x)$ lösen lässt: $z(x, t)=h(x) \cdot g(t)$. Damit ist

$$
c \cdot g(t) \frac{\mathrm{d}^{4}}{\mathrm{dx}^{4}} h(x)-\mu \cdot h(x) \frac{\mathrm{d}^{2}}{\mathrm{dt}^{2}} g(t)-D \cdot h(x) \frac{\mathrm{d}}{\mathrm{dt}} g(t)=0
$$

oder

$$
\frac{1}{h(x)} \cdot \frac{c}{\mu} \frac{\mathrm{d}^{4}}{\mathrm{dx}^{4}} h(x)=\frac{1}{g(t)} \cdot\left(\frac{\mathrm{d}^{2}}{\mathrm{dt}^{2}} g(t)+\frac{D}{\mu} \cdot \frac{\mathrm{d}}{\mathrm{dt}} g(t)\right)=: \omega_{0}^{2} .
$$

Da die linke Seite nur vom Ort und die rechte Seite nur von der Zeit abhängt, können beide Seiten gleich einer konstanten $\omega_{0}^{2}$ gesetzt werden. Der zeitabhängige Teil hat nun die Form

$$
\frac{\mathrm{d}^{2}}{\mathrm{dt}^{2}} g(t)+\frac{D}{\mu} \frac{\mathrm{d}}{\mathrm{dt}} g(t)-\omega_{0}^{2} g(t)=0
$$


wie sie für harmonische Schwingungen bekannt ist, und lässt sich lösen durch

$$
g(t)=A_{0} e^{-\lambda t} \cos \omega t
$$

mit $\lambda=D / 2 \mu$ und $\omega=\sqrt{\omega_{0}^{2}-\lambda^{2}}$. Diese Gleichung beschreibt eine harmonische Schwingung mit der Frequenz $f=\omega / 2 \pi$ und exponentiell abklingender Amplitude $A_{0}$. Der ortsabhängige Teil der Bewegungsgleichung

$$
\frac{c}{\mu} \frac{\mathrm{d}^{4}}{d x^{4}} h(x)-\omega_{0}^{2} h(x)=0
$$

lässt sich ebenfalls mit einer Superposition reeller und komplexer Exponentialfunktionen lösen. Da die Auslenkung $h(x)$ reellwertig sein soll, bietet sich die Schreibweise

$$
h(x)=B \sin (k x)+C \cos (k x)+E \sinh (k x)+F \cosh (k x)
$$

mit $k=\left(\omega_{0}^{2} \mu / c\right)^{1 / 4}$ an [75]. Da der Balken einseitig bei $x=0$ fest eingespannt ist, kann er hier weder ausgelenkt noch verkippt werden, so dass $h(0)=\frac{\mathrm{d}}{\mathrm{dx}} h(0)=0$ gilt, während er am freien Ende nicht gekrümmt ist, so dass zweite und dritte Ableitung verschwinden. Damit haben wir vier Randbedingungen, so dass $B, C, E$ und $F$ bestimmt werden können. Es ergibt sich

$$
B=-E \quad C=-F \quad \text { und } \quad B=C \cdot \frac{\sin (k L)-\sinh (k L)}{\cos (k L)+\cosh (k L)}
$$

Dabei ist zu beachten, dass nichttriviale Lösungen nur existieren, wenn die Determinante des Gleichungssystems zur Bestimmung von $E$ und $F$ aus der zweiten und dritten Ableitung verschwindet, d.h. für

$$
1+\cos (k L) \cosh (k L)=0 .
$$

Diese Gleichung ist numerisch lösbar und liefert als ersten Wert $k L=1,8751$ [77], womit Gleichung $3.15 \mathrm{zu}$

$$
h(x)=B \cdot(\sin (k x)-1,275 \cos (k x)-\sinh (k x)+1,275 \cosh (k x))
$$

wird und die Kreisfrequenz der ersten Schwingungsmode berechnet werden kann:

$$
\omega_{0}=\sqrt{\frac{c}{\mu}} k^{2}=\frac{1,875^{2}}{L^{2}} \sqrt{\frac{E d^{2}}{12 \rho}} .
$$




\section{Konzeption des Aufbaus}

Grundsätzlich sind Vibrating-Reed-Experimente eine etablierte Technik [78]. Der Aufbau einer eigenen Anlage bietet jedoch die Möglichkeit, diese speziell für die Anforderungen der eigenen Proben zu optimieren. So unterscheiden sich die Ansprüche an die Einspannung für metallische Folien grundsätzlich von denen z. B. für einkristalline Siliziumplättchen. Auch Heizung und Kühlung können speziell auf den relevanten Temperaturbereich zugeschnitten werden. Bei der Konzeption eines solchen Aufbaus sind allgemein verschiedene Punkte zu beachten:

\section{Heizen und Kühlen}

Je nach Zusammensetzung zeigt Eisen-Palladium knapp ober- oder unterhalb von Zimmertemperatur den martensitischen Phasenübergang. Um diesen eingehend zu untersuchen, soll die Probe sowohl geheizt als auch gekühlt werden. Dabei genügt ein Bereich von ca $-70{ }^{\circ} \mathrm{C}$ bis $+150{ }^{\circ} \mathrm{C}$. Da die Messungen nach Möglichkeit im thermischen Gleichgewicht stattfinden sollen, welches sich bei diesen Temperaturen nur langsam einstellt, werden geringe Heizraten benötigt. Zur Minimierung von Temperaturschwankungen ist außerdem ein Aufbau mit hoher Wärmekapazität vorteilhaft.

Um diesen Anforderungen gerecht zu werden, wird die Probenhalterung an einem massiven Kupferblock befestigt, der erst erwärmt bzw. abgekühlt werden muss, bevor sich die Probentemperatur signifikant ändert. Der Kupferblock kann mittels eines SiliziumNitrid-Heizers mit einem regelbaren Netzteil geheizt werden. Die Kühlung erfolgt mit Hilfe von flüssigem Stickstoff, der in einen kleinen isolierten Tank eingefüllt wird. Ein Kühlfinger mit hoher Wärmekapazität, der mittels breiter Kupferlitze thermisch an den Kupferblock angekoppelt ist, sorgt für eine gleichmäßige Kühlung der Probe, auch nachdem der Stickstoff verdampft ist. Dies ist besonders wichtig, da das Sieden des Stickstoffes zu Schwingungen des Aufbaus führt, die die Messung empfindlich stören würden.

Die Bestimmung der Probentemperatur erfolgt mit Hilfe eines Thermoelementes vom Typ K. Für eine genaue Temperaturbestimmung wäre es am besten, das Thermoelement direkt auf der Probe zu befestigen. Dies würde jedoch ihre Schwingungseigenschaften dramatisch beeinflussen. Deshalb wurde eine Position direkt an der Einspannung auf der dem Heizer und Kühlfinger abgewandten Seite gewählt. So wird eine bestmögliche Übereinstimmung von Probentemperatur und gemessener Temperatur gewährleistet.

\section{Thermische Ankopplung}

Für temperaturabhängige Messungen muss es möglich sein, die Probe gezielt zu heizen bzw. zu kühlen und dabei ihre Temperatur zu bestimmen. Da die Probe schmal und dünn ist und nur an einer Seite Kontakt zum Rest des Aufbaus hat, kann Wärmeleitung nur eingeschränkt genutzt werden, während die Empfindlichkeit der Probe gegenüber Wärmestrahlung recht hoch ist. Bei den für die Legierung Eisen-Palladium interessanten Temperaturen um und unterhalb von Zimmertemperatur wird jedoch nur 
sehr wenig Wärme abgestrahlt. Um beide Effekte optimal zu nutzen, wurde der Aufbau so gestaltet, dass die gesamte Umgebung der Probe geheizt bzw. gekühlt wird. Damit gleicht sich nach einiger Zeit die Probentemperatur der Umgebungstemperatur an. Dazu wurde zwischen Heizer bzw. Kühlfinger und Probe der oben erwähnte massive Kupferblock eingebaut. Wie in Abbildung 3.6 ersichtlich, kann die Probe durch eine Kupferhaube noch weiter gegen Wärmestrahlung abgeschirmt werden. Das Angleichen von Proben- und Umgebungstemperatur, verbunden mit niedrigen Heizraten von 0,1 bis $0,5^{\circ} \mathrm{C}$ pro Minute, stellt außerdem eine gleichmäßige Abkühlung bzw. Erwärmung der Probe auf ihrer gesamten Länge sicher.

\section{Einspannung}

Ein besonderes Augenmerk muss auf die Einspannung als Berührungspunkt von Apparatur und Probe gerichtet werden. Da der Kraftübertrag zwischen Probe und Einspannung an der Vorderkante maximal ist, muss sie aus einem möglichst harten Material beschaffen sein, damit sie sich nicht durch die Schwingung der Probe verformt, außerdem sollte die Einspannung möglichst starr gefertigt sein, damit sie nicht mit der Probe mitschwingt und dabei deren Schwingung dämpft. Des Weiteren sollte die Vorderkante der Einspannung scharf und gerade sein, so dass die Probenlänge klar definiert ist, da sie in vierter Potenz in die Berechnung des Elastizitätsmoduls eingeht. Außerdem muss eine Verspannung oder gar Verformung der Probe parallel zur Vorderkante der Einspannung vermieden werden, da sie die Werte für den Elastizitätsmodul ebenfalls stark verfälschen würde. Bei temperaturabhängigen Messungen ist auch die thermische Ausdehnung zu beachten. Wenn sich die Ausdehnungskoeffizienten von Probe und Einspannung zu stark unterscheiden, lockert sich beim Kühlen bzw. Heizen die Halterung, was wiederum zu erhöhter Dämpfung führt.

Um die genannten Voraussetzungen zu erfüllen, wurde die Probeneinspannung aus Edelstahl gefertigt. Wie in Abbildung $3.6 \mathrm{zu}$ sehen, besteht die Probeneinspannung aus einem massiven Block, auf dem mit zwei Schrauben eine Deckplatte befestigt wird. Die Flächen sowie die Vorderkante wurden glatt gefräst und ein Verschieben der Einspannungsteile gegeneinander durch zwei Führungsstifte verhindert. Die Probe wird zwischen Block und Deckplatte geklemmt. Dabei ist darauf zu achten, dass beide Schrauben gleichmäßig angezogen werden. Da die Probeneinspannung komplett aus der Kammer herausgenommen werden kann, ist ein sorgfältiger, sehr vorsichtiger Einbau der Probe ohne nennenswerte Querverspannung sowie ein genaues Messen der Probenlänge möglich. Um Einflüsse der apparativen Dämpfung weiter zu reduzieren, wurde die Edelstahleinspannung für Dämpfungsmessungen mit zwei Siliziumplättchen belegt, deren intrinsische Dämpfung noch geringer ist als die von Edelstahl. Der Edelstahlblock wird in eine Vertiefung des oben erwähnten Kupferblocks eingelassen und mit zwei Madenschrauben fixiert, so dass neben einem guten Wärmeübergang auch eine reproduzierbare Probenposition sichergestellt ist.

\section{Anregung}

Sollen Resonanzkurven aufgenommen werden, so muss die Anregung der Schwingung berührungslos erfolgen, um das Schwingungsverhalten der Probe nicht zusätzlich zu 
beeinflussen. Sollen außerdem Abklingkurven aufgenommen werden, ist es wichtig, dass die Anregung schnell abgeschaltet werden kann. Für beide Anordnungenn bietet sich eine kapazitive Anregung an. Die Probe selbst bildet mit einer Elektrode in der Nähe der Probenspitze einen Kondensator. Liegt zwischen den Kondensatorplatten der Fläche $A$ im Abstand $s$ eine Spannung $U$ an, so wirkt eine anziehende Kraft, mit der die Probe aus ihrer Ruhelage ausgelenkt werden kann:

$$
F=\frac{1}{2} \frac{\epsilon_{0} A}{s^{2}} U^{2}
$$

Da die Dielektrizitätszahl $\epsilon_{0}$ sehr klein ist, sind die elektrostatischen Kräfte zwischen den Kondensatorplatten auch verhältnismäßig klein, so dass auf diese Weise im Allgemeinen keine hinreichend große Auslenkung erreicht wird, um beim Abschalten der Spannung die Probe in eine messbare Schwingung zu versetzen. Deshalb erfolgt die Anregung mit Hilfe einer Wechselspannung geeigneter Frequenz nahe der Resonanzfrequenz der Probe. Insbesondere wenn Resonanzkurven aufgenommen werden, ist es wichtig, die Wechselspannung sehr genau und störungsarm regeln zu können. Dazu wurde eine in den Messrechner eingebaute DA-AD-Wandlerkarte der Firma NATIONAL Instruments ${ }^{(2)}$ verwendet. Mit einer gegebenen Rate (in diesem Fall 1000 Punkte pro Sekunde) wird ein Sinussignal mit einstellbarer Amplitude modelliert, das von der DA-AD-Wandlerkarte als Wechselspannung ausgegeben wird. Die Anregung kann auf zweierlei Arten geschehen:

Wird einfach eine Wechselspannung $U(t)=U_{0} \cos (\omega t)$ angelegt, wirkt zwischen Probe und Elektrode die Kraft

$$
F=\frac{1}{4} \frac{\epsilon_{0} A}{s^{2}} U_{0}^{2}(1+\cos (2 \omega t))
$$

da die Spannung quadratisch eingeht. Das heißt, die Kraft oszilliert mit der doppelten Frequenz der Spannung. Da die Ausgangsspannung der DA-AD-Wandlerkarte auf $\pm 10 \mathrm{~V}$ begrenzt ist, wirkt bei einer Elektrodenfläche von ca. $25 \mathrm{~mm}^{2}$ und einem Abstand $s$ von $1 \mathrm{~mm}$ maximal eine Kraft von etwa $2 \cdot 10^{-9} \mathrm{~N}$. Dies reicht in vielen Fällen nicht aus, um die Probe in Schwingung zu versetzen. Dieses Problem kann gelöst werden, indem die Wechselspannung mit einer Gleichspannung überlagert wird. Die Gleichspannung muss sehr konstant sein, insbesondere darf sie keine Wechselstromanteile enthalten, die beispielsweise beim Gleichrichten der Netzspannung übrig bleiben. Deshalb bietet es sich an, eine Batteriebox als Gleichspannungsquelle zu benutzen. Drei Batterien der Firma Everready mit einer Ausgangsspannung von je 66,6 V wurden in Reihe geschaltet, so dass eine Gleichspannung $U_{\text {Bias }}$ von $200 \mathrm{~V}$ zur Verfügung steht. Auf diese Gleichspannung wird eine Wechselspannung von bis zu $10 \mathrm{~V}$ aufmodelliert. Damit wirkt eine Kraft

$$
\begin{aligned}
F & =\frac{1}{2} \frac{\epsilon_{0} A}{s^{2}}\left(U_{\text {Bias }}+U_{0} \cos (\omega t)\right)^{2} \\
& =\frac{1}{2} \frac{\epsilon_{0} A}{s^{2}}\left(U_{\text {Bias }}^{2}+2 U_{\text {Bias }} U_{0} \cos (\omega t)+U_{0}^{2} \cos ^{2}(\omega t)\right) .
\end{aligned}
$$

${ }^{(2)}$ Multifunction DAQ vom Typ NI 6040E 
Da die Gleichspannung $U_{\text {Bias }}$ wesentlich größer ist als die Amplitude $U_{0}$ der Wechselspannung, und damit $2 U_{\text {Bias }} U_{0} \gg U_{0}^{2}$, ergibt sich in erster Näherung eine oszillierende Kraft von

$$
F \approx \frac{\epsilon_{0} A}{s^{2}} U_{\text {Bias }} \cdot U_{0} \cos (\omega t)
$$

sowie eine statische Kraft, welche die Schwingung nicht weiter beeinflusst. Mit der Gleichspannung kann eine hundertmal so große mit der gleichen Frequenz wie die Wechselspannung oszillierende Kraft von etwa $2 \cdot 10^{-7} \mathrm{~N}$ erreicht werden. Für die Messung von Abklingkurven kann die Wechselspannung einfach zu einem definierten Zeitpunkt abgeschaltet werden. Die Gleichspannung bleibt bestehen und beeinflusst die Schwingung nicht signifikant, sofern die Auslenkung deutlich kleiner bleibt als der Abstand zur Elektrode.

\section{Signalerfassung}

Das Auslesen der Schwingungsamplitude muss in jedem Fall berührungslos erfolgen, um das Schwingungsverhalten der Probe nicht zu beeinflussen. Oft wird die Auslenkung kapazitiv mit einer zweiten Elektrode erfasst. Diese Methode hat jedoch mehrere Nachteile. Zum einen gilt es, Streukapazitäten und -induktivitäten im Messkreis zu vermeiden, insbesondere da die Ströme, die aufgrund der Änderung der Kapazität fließen, sehr klein sind. Zum anderen besteht die Gefahr des sogenannten Übersprechens zwischen den Elektroden, d.h. die Anregungselektrode verursacht direkt eine Spannungsänderung an der Messelektrode, unabhängig von der Bewegung der Probe. Dieses Übersprechen ist schwer von einem echten Signal zu unterscheiden, da es mit der gleichen Frequenz wie die Anregung bzw. die Schwingung erfolgt.

Um diese Probleme zu vermeiden, wird die Probenbewegung optisch erfasst. Ein Laserstrahl ist auf die Probe gerichtet, wird reflektiert und fällt auf einen positionssensitiven Detektor (PSD) (siehe Abbildung 3.4), der aus halbleitendem Material besteht, das bei Lichteinfall Ladungsträger freisetzt. So entsteht ein Fotostrom, der an den Rändern des Detektors abfließst. Das Verhältnis der Ströme $I_{A}$ und $I_{B}$ am rechten und linken Rand ist proportional zur Entfernung des Lichtpunktes vom jeweiligen Kontakt; ihre Größe hängt von der Intensität des einfallenden Lichts ab. Eine analoge Elektronik wandelt die Stromsignale in Spannungssignale $U_{A}$ und $U_{B}$ um, bildet die Differenz $U_{A}-U_{B}$ und normiert dieses Signal bezüglich der Gesamtintensität $U_{A}+U_{B}$. Mit einer einstellbaren Rate wird das normierte Spannungssignal in Echtzeit über die DA-AD-Wandlerkarte in den Messrechner eingelesen. Da die Resonanzfrequenzen der Proben in der Regel zwischen $60 \mathrm{~Hz}$ und $100 \mathrm{~Hz}$ lagen, war eine Rate von 1000 Werten pro Sekunde (d.h. 10 bis 15 Stützstellen je Schwingung) ausreichend.

Abbildung 3.6 zeigt noch einmal als Gesamtbild, wie die oben genannten Anforderungen umgesetzt wurden. Um die apparative Dämpfung zu minimieren, befindet sich der Aufbau in einer Vakuumkammer, die auf einen Druck unter $10^{-6}$ mbar evakuiert wird. Damit ist die Dämpfung durch Luftreibung zu vernachlässigen [79]. Die Proben sind in der Regel ca. $5 \mathrm{~mm}$ breit, $0,05 \mathrm{~mm}$ dick und haben eine Länge $L$ von $20 \mathrm{~mm}$. Der Abstand zwischen Probe und Positionsdetektor, d.h. die Länge $l$ des Lichtzeigers, beträgt etwa $40 \mathrm{~cm}$. Die während einer Schwingung vom reflektierten Laserpunkt auf dem 


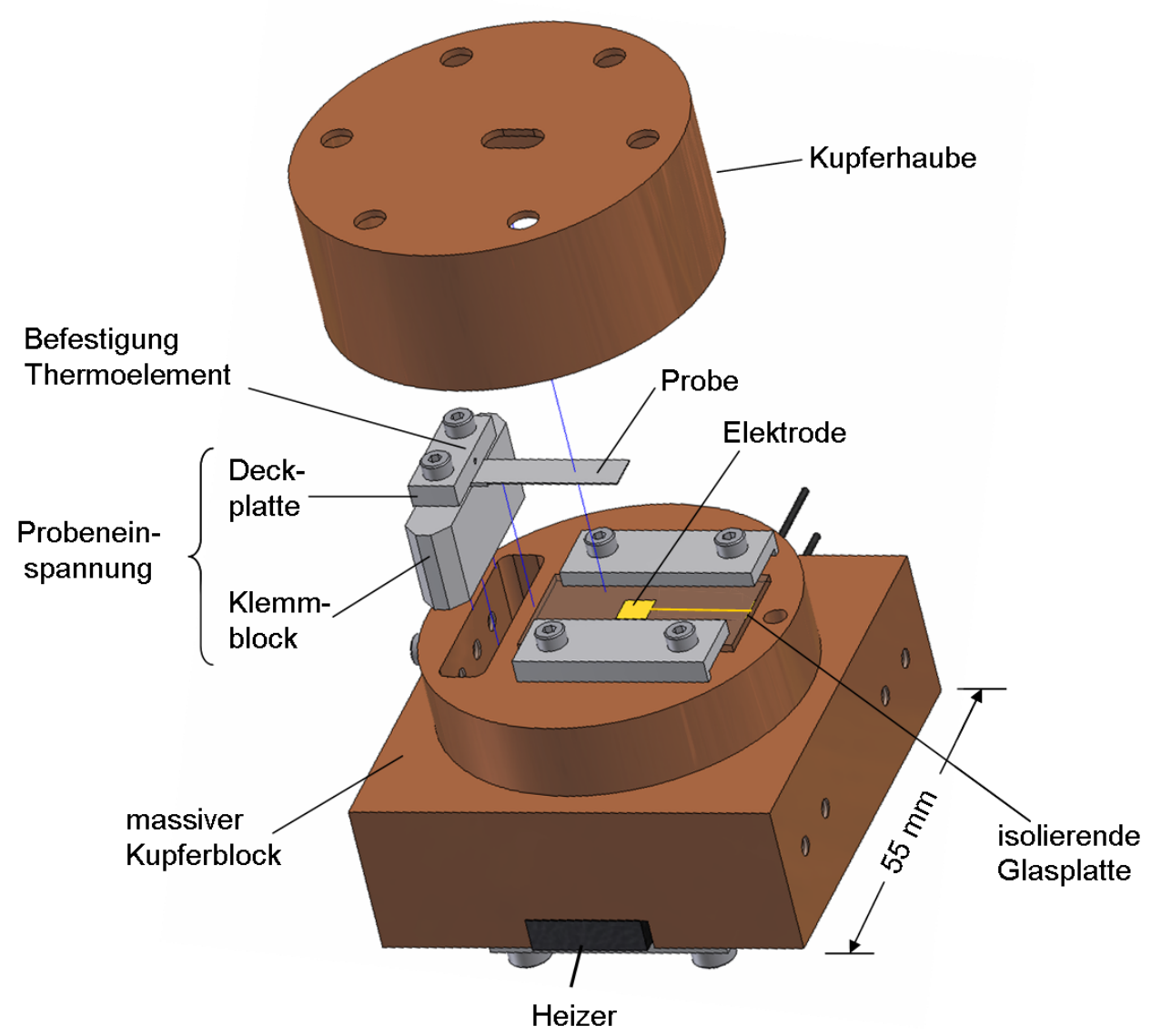

Abbildung 3.6: Die Einspannung besteht aus einem massiven Block, auf dem mit zwei Schrauben eine Deckplatte befestigt werden kann. Der Edelstahlblock wird in eine Vertiefung des Kupferblocks eingelassen auf dessen Rückseite der Heizer befestigt ist.

Detektor zurücklegte Strecke $y$ war stets kleiner als $1 \mathrm{~cm}$. Damit kann die maximale Auslenkung $h(L)$ der Probe aus ihrer Ruhelage abgeschätzt werden:

Für die Auslenkung des Laserpunktes ist die Neigung $\frac{\mathrm{d} h}{\mathrm{~d} x}$ des Probenendes, an dem der Strahl reflektiert wird, gegenüber der Ruhelage entscheidend: $y=l \cdot \frac{\mathrm{d} h}{\mathrm{~d} x}$. Die Neigung des Probenendes kann durch Ableiten von Gleichung 3.18 gewonnen werden:

$$
\left.\frac{\mathrm{d} h}{\mathrm{~d} x}\right|_{x=L}=B k(\cos k L+1,275 \sin k L-\cosh k L-1,275 \sinh k L)=-12,15 \frac{B}{L},
$$

unter Berücksichtigung, dass $k L=1,8751$ ist. Nun kann $B$ bestimmt werden:

$$
B=-\frac{\mathrm{d} h}{\mathrm{~d} x} \cdot \frac{L}{12,15}=-\frac{y}{l} \cdot \frac{L}{12,15} .
$$

Die maximale Auslenkung des Probenendes ergibt sich nun direkt durch Einsetzen in Gleichung 3.18:

$$
h(L)=B \cdot(\sin (k L)-1,275 \cos (k L)-\sinh (k L)+1,275 \cosh (k L)) \approx 1 \cdot 10^{-4} \mathrm{~m} .
$$

Dieser Wert ist sehr viel kleiner als die Probenlänge oder der Abstand zwischen Probe und Elektrode. Die Näherung kleiner Auslenkungen ist also in jedem Fall gültig. 


\section{Ablauf der Messung}

Nach dem Einbau einer Probe muss zunächst näherungsweise ihre Resonanzfrequenz bestimmt werden, um sie zu Schwingungen mit großer Amplitude anregen zu können. Erst dann können aus den Abklingkurven Eigenfrequenz und Dämpfung ermittelt werden. Die Vibrating-Reed-Apparatur kann also in zwei verschiedenen Modi betrieben werden: der Suche nach der Resonanzfrequenz und der eigentlichen Messung. Für die beiden Modi wurden mit LABVIEW zwei unabhängige Messprogramme erstellt.

Bei der Suche nach der Resonanzfrequenz wird zunächst die Probentemperatur eingelesen. Anschließend wird die Probe mit einer bestimmten Frequenz angeregt und gleichzeitig das zu ihrer Bewegung proportionale Spannungssignal über mehrere Sekunden aufgenommen. Im nächsten Schritt wird wieder die Temperatur eingelesen, die Anregungsfrequenz um die gewünschte Schrittweite erhöht und die Auslenkung protokolliert. Bei jedem Schritt wird die Differenz zwischen dem höchsten Maximum und dem tiefsten Minimum des Signals erfasst. Dieser Wert ist eine gute Näherung für die Amplitude der Schwingung, so dass eine Resonanzkurve aufgenommen wird. Abhängig von der eingestellten Schrittweite, erhält man so einen mehr oder weniger genauen Näherungswert für die Resonanz- und damit auch für die Eigenfrequenz.

Damit kann die eigentliche Messung gestartet werden. Die Probe wird mit einer Frequenz nahe ihrer Resonanzfrequenz zu Schwingungen angeregt. Dann wird die Anregung abgeschaltet und aus der Frequenz der freien Schwingung die Eigenfrequenz der Probe gewonnen. Nun wird sie mit dieser Frequenz angeregt, bis die Amplitude der Schwingung näherungsweise konstant ist. Währenddessen wird die Temperatur eingelesen. Wird nun die Anregung abgestellt, kann aus der Abklingkurve der freien Schwingung, die jetzt eine größere Amplitude hat, ein genauerer Wert für die Eigenfrequenz und die Dämpfung der Probe gewonnen und in eine Datei geschrieben werden. Ein Messzyklus (wie in Abbildung 3.7 dargestellt) dauert etwa eine Minute, wobei die eigentliche Messung während der zweiten Abklingkurve nur 5 Sekunden in Anspruch nimmt, so dass sich bei typischen Heiz- und Kühlraten von $0,1^{\circ} \mathrm{C}$ bis $0,4^{\circ} \mathrm{C}$ pro Minute die Temperatur während der Messung nicht signifikant ändert.

Für den nächsten Messzyklus wird nicht die aus der Abklingkurve des letzten Zyklus gewonnene Eigenfrequenz, sondern zunächst wieder der Näherungswert für die Resonanzfrequenz benutzt. So kann die Messung auch nach einer Störung, die einmalig eine falsche Eigenfrequenz liefert, weiterlaufen. Es muss lediglich auf einen Messpunkt verzichtet werden. Andererseits muss auch bei großen Änderungen der Eigenfrequenz der Näherungswert für die Anregung nur gelegentlich nachgeregelt werden, da wegen des zweiteiligen Messzyklus die Proben stets mit der optimalen Frequenz angeregt werden.

Wie im vorherigen Abschnitt erwähnt, werden Eigenfrequenz $f_{0}$ und Dämpfung aus den Abklingkurven der freien Schwingung ermittelt. Dazu wird das Response-Signal mit einer Rate von 1000 Punkten pro Sekunde eingelesen. Mit Hilfe der Fast Fourier Transformation (FFT) wird die Frequenz der Schwingung ermittelt. Dazu steht ein vorgefertigtes LABVIEW-Werkzeug zur Verfügung.

Um die Dämpfung zu ermitteln, wird zunächst mit einem vorgefertigten LABVIEWWerkzeug der zeitliche Mittelwert der Amplitude bestimmt und abgezogen, um eine Oszillation um Null zu erhalten. Nun werden die Positionen und Amplituden der Maxima bzw. Minima bestimmt. An die logarithmierten Werte der Extrema wird eine 

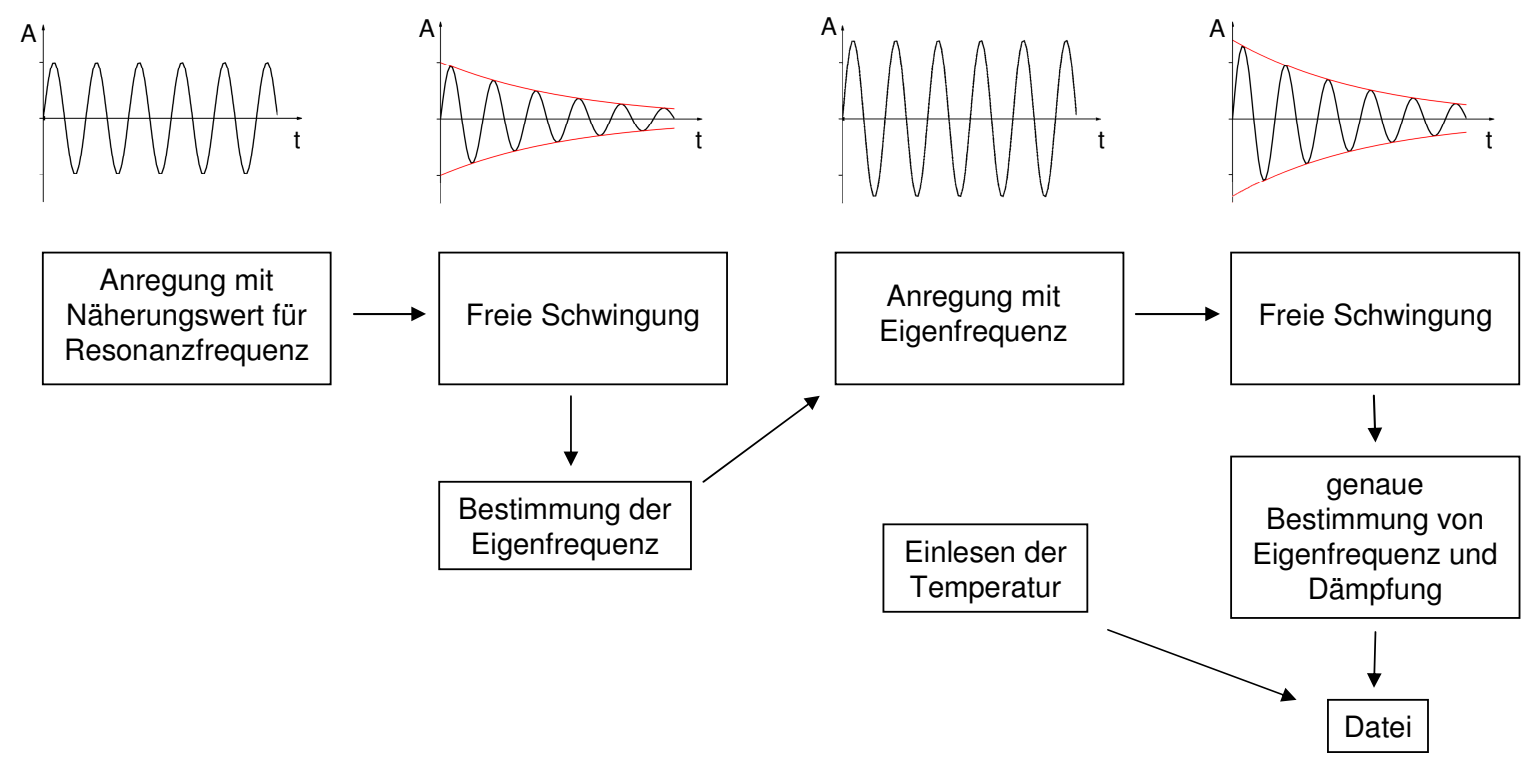

\begin{abstract}
Abbildung 3.7: Zunächst wird die Probe mit einer Frequenz nahe ihrer Resonanzfrequenz zu Schwingungen angeregt und aus der Frequenz der freien Schwingung ihre Eigenfrequenz gewonnen, mit der sie erneut angeregt wird. Aus der Abklingkurve der zweiten freien Schwingung kann ein genauerer Wert für die Eigenfrequenz und die Dämpfung der Probe gewonnen werden.
\end{abstract}

Gerade angepasst, aus deren Steigung $\lambda$ das logarithmische Dekrement und damit die Dämpfung $\lambda$ gewonnen wird:

$$
Q^{-1}=\frac{\lambda}{\pi f}
$$

Für jede Temperatur werden die Eigenfrequenz, die aus den Maxima und Minima berechnete Dämpfung und deren Fehler sowie die maximale Amplitude in eine Datei geschrieben. Zur Kontrolle werden zusätzlich die gesamten Abklingkurven in separaten Dateien gespeichert. 


\section{Kapitel 4}

\section{Ergebnisse}

In diesem Teil der Arbeit werden die Ergebnisse gezeigt, die mit den in Kapitel 3 vorgestellten Methoden gewonnen wurden. Nach einer kurzen Charakterisierung der makroskopischen und mikroskopischen Struktur der Splats befasst sich der Abschnitt 4.2 mit dem Einfluss von Zusammensetzung und Glühbehandlung auf die bei Zimmertemperatur auftretenden Phasen und ihre Stabilität. Der Abschnitt 4.3 konzentriert sich auf den Übergang von der austenitischen zur martensitischen Phase. Dabei sind die Änderung der strukturellen, magnetischen und mechanischen Probeneigenschaften mit der Temperatur und ihre Abhängigkeit von der Mikrostruktur von besonderem Interesse.

\subsection{Makroskopische und mikroskopische Struktur}

Beim Zusammenschlagen der Kupferstempel des Splatquenchers wird das Material sehr schnell abgekühlt (siehe Abbildung 3.1). Gleichzeitig wird das ursprünglich runde Materialkügelchen mechanisch massiv verformt, da das Material zu den Seiten verdrängt wird. Dabei kristallisiert es von der Mitte nach außen, wodurch eine charakteristische Struktur mit deutlichen Unterschieden zwischen der Mitte und dem Rand des Spalts entsteht, die in Abbildung 4.1 zu erkennen sind. Außerdem entsteht ein Spannungsgradient zwischen der Mitte und den äußeren Bereichen der Splats, so dass sich diese leicht wölben. Da das Material während der mechanischen Verformung noch flüssig ist, kann es einfach verdrängt werden, so dass die Splats zwei glatte Oberflächen erhalten und bei einem Durchmesser von ca. $2 \mathrm{~cm}$ typischerweise eine Dicke von nur $50 \mu \mathrm{m}$ aufweisen.

Da die im Material gespeicherte Wärme über die Kupferstempel schnell abgeführt wird, beginnt das Material an beiden Oberflächen, die mit den Kupferstempeln Kontakt haben, zu kristallisieren. Wie in Abbildung 4.1 zu erkennen, wachsen dabei von beiden Oberflächen aus Säulen, die sich in der Mitte treffen. Anhand der REM-Aufnahmen in Abbildung 4.2 kann abgeschätzt werden, dass die Säulen in unbehandelten Proben zwischen $150 \mathrm{~nm}$ und $200 \mathrm{~nm}$ breit sind. Die TEM-Aufnahmen in Abbildung 4.3 und 4.4 zeigen, dass in geglühten Proben die Breite deutlich ansteigt. Nach dem Glühen bei $800{ }^{\circ} \mathrm{C}$ beträgt die mittlere Säulenbreite etwa $400 \mathrm{~nm}$, nach dem Glühen bei $1000{ }^{\circ} \mathrm{C}$ liegt sie zwischen $500 \mathrm{~nm}$ und $600 \mathrm{~nm}$. 

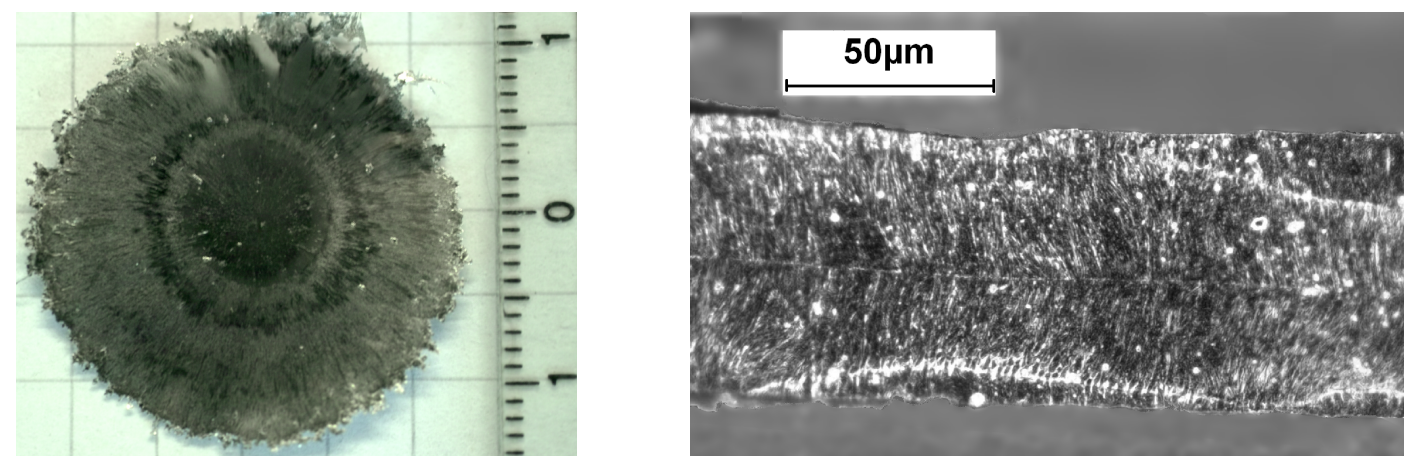

Abbildung 4.1: Splats haben einen Durchmesser von ca. $2 \mathrm{~cm}$ und sind etwa $50 \mu \mathrm{m}$ dick. In der Lichtmikroskop-Aufnahme des Querschnitts ist die Säulenstruktur erkennbar.

Wegen der hohen Abschreckgeschwindigkeit ist das Material sehr reich an Defekten. Dies äußert sich in der geringen Kohärenzlänge der reflektierten Röntgenstrahlung, die zu einer Verbreiterung der Peaks führt, wie sie in den Diffraktogrammen in Abschnitt $4.2 \mathrm{zu}$ sehen ist. In diesem Fall ist es jedoch nicht ohne Weiteres möglich, die Korngröße $B$ über die Wellenlänge $\lambda$ und den Streuwinkel $\theta$ mit Hilfe der Scherrerformel [80]

$$
B=\frac{0,9 \lambda}{w \cos \theta}
$$

mit der Peakbreite $w$ zu verknüpfen, da auch lokale Gitterverzerrungen die Peakbreite beeinflussen. Dieses Problem wird in Abschnitt 5.4.3 genauer erörtert. Aus der Peakverbreiterung können lediglich Rückschlüsse auf die Qualität der Kristallstruktur des Materials, also auf den Grad der Verzerrung sowie auf die Menge der Defekte wie Leerstellen, Versetzungen oder Korngrenzen gezogen werden.

In der Regel tritt bei gerichteter Erstarrung von polykristallinem Material eine Vorzugsorientierung der Kristallite, eine Textur auf. Diese kann mit Hilfe der in Abschnitt 3.2.1 beschriebenen Vierkreis-Röntgenmessungen nachgewiesen werden. In Abbildung 4.5 ist eine (111)-Polfigur zu sehen, die aus einer entsprechenden Messung an einer ungetemperten Probe gewonnen wurde. Die leichte Erhöhung der Intensität in der Mitte der Aufnahme für $\psi=0$ kann mit dem Anstieg des bestrahlten Probenvolumens für kleine Kippwinkel erklärt werden. Im Wesentlichen ist die Intensität also für alle Probenorientierungen gleich, es ist keine Textur vorhanden.

Dennoch sind die Proben nicht in jeder Hinsicht isotrop. Zwar sind im Austenit alle Kristallorientierungen vorhanden, im Martensit treten jedoch nicht alle Varianten gleichermaßen auf. Dies ist in Röntgendiffraktometrie-Messungen in Bragg-BrentanoGeometrie zu sehen, die an Proben in der martensitischen und austenitischen Phase durchgeführt wurden. Aufgrund der tetragonalen Verzerrung der Einheitszelle beim Phasenübergang ist eigentlich ein Aufspalten des fcc(200)-Peaks in einen fct(200)- und einen fct(002)-Peak zu erwarten. In der Messung in Abbildung 4.6 tritt jedoch nur der fct(200)-Peak auf. Das bedeutet, dass vorzugsweise diejenigen Martensitvarianten entstehen, deren kurze Achse parallel zur Probenebene orientiert ist. Dieses Verhalten wird an fast allen transformierenden Proben beobachtet. Lediglich in einer Probe tritt auch der (002)-Peak auf. 


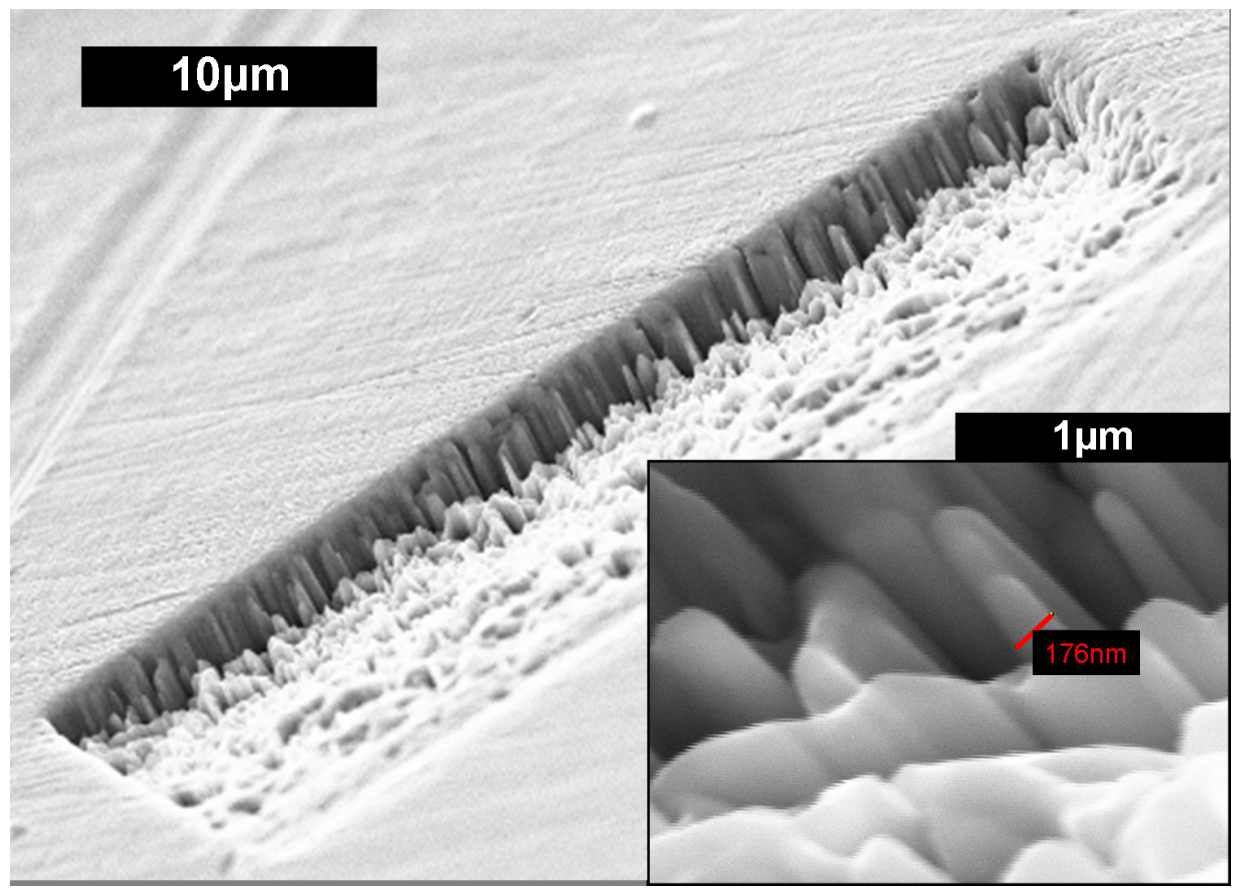

Abbildung 4.2: Mit dem FIB wurde ein Graben in eine ungetemperte Probe präpariert. Anhand der REM-Bilder lässt sich abschätzen, dass die Säulenbreite zwischen $150 \mathrm{~nm}$ und $200 \mathrm{~nm}$ liegt. (FIB: H.Brunken, REM: S.Hamann)
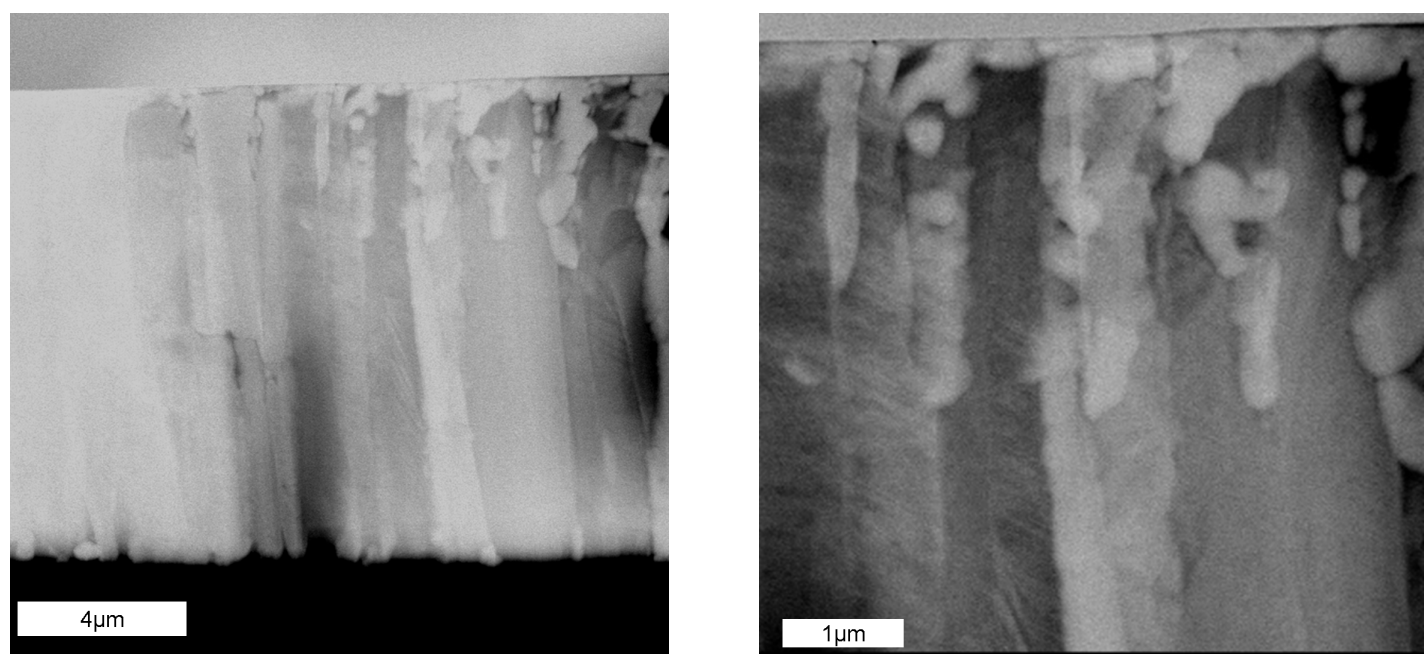

Abbildung 4.3: Diese TEM-Aufnahme einer bei $800^{\circ} \mathrm{C}$ geglühten Probe zeigt die Säulenstruktur noch deutlicher. Teilweise ist ein charakteristisches Fischgrätenmuster zu erkennen. Die mittlere Säulenbreite beträgt ca. 400 nm. (FIB: H.Brunken, TEM: D.König) 


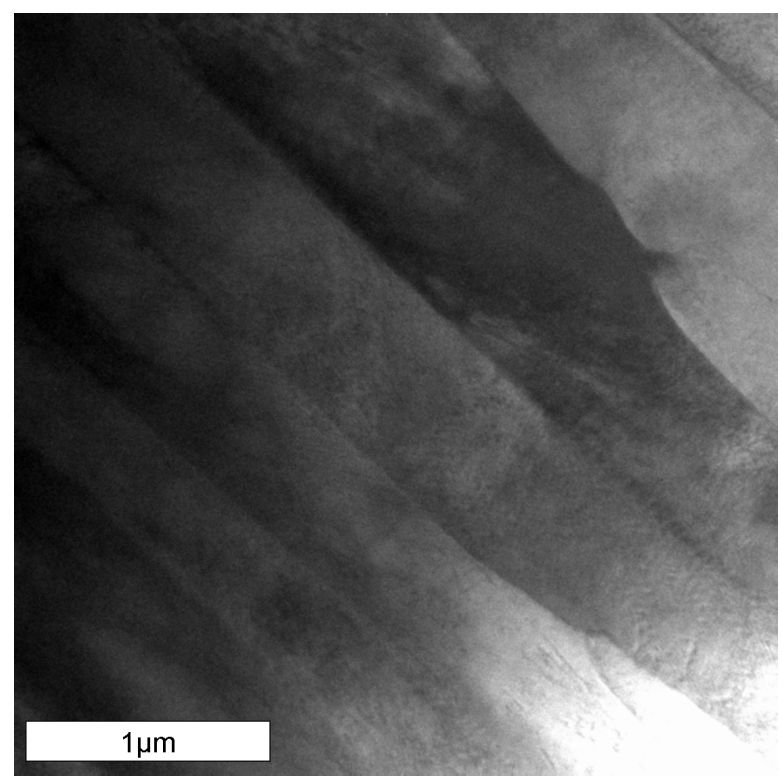

Abbildung 4.4: In dieser TEM-Aufnahme einer bei $1000^{\circ} \mathrm{C}$ geglühten Probe ist die Säulenstruktur am stärksten ausgeprägt. Die Säulenbreite liegt zwischen $500 \mathrm{~nm}$ und $600 \mathrm{~nm}$. (FIB: H.Brunken, TEM: D.König)

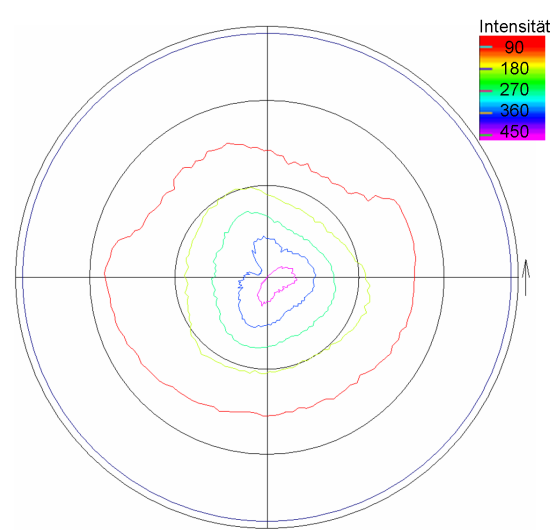

Abbildung 4.5: Die (111)-Polfigur zeigt keine nennenswerte Textur.

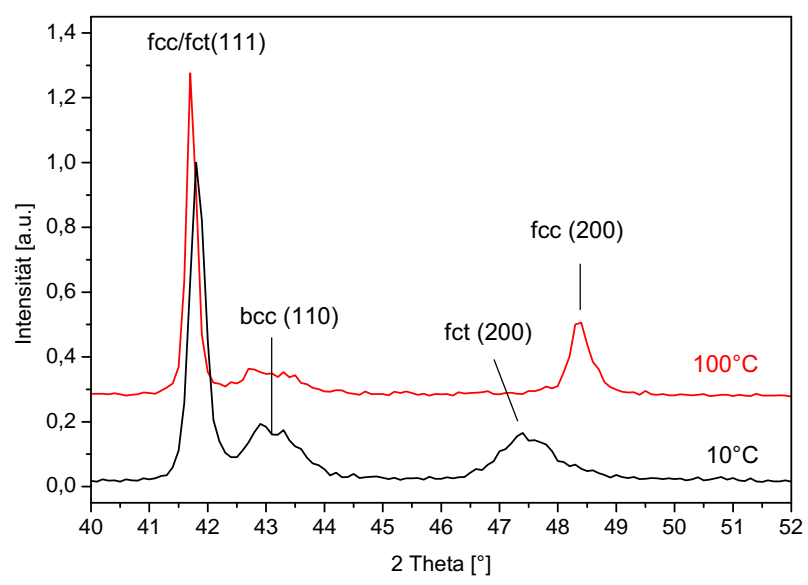

Abbildung 4.6: Statt einer Aufspaltung ist nur eine Verschiebung des (200)Röntgenpeaks zu beobachten, es tritt also nur eine Martensitvariante auf. 


\subsection{Phasen und ihre Stabilität}

Wie zuvor beschrieben, können mit der Splatquenching-Technik aufgrund der hohen Abkühlraten die metastabilen Hochtemperaturphasen des Legierungssystems bei Zimmertemperatur bewahrt werden. Doch welche der metastabilen Phasen sich einstellt und ob das System bei einer nachfolgenden Glühbehandlung zum Entmischen neigt, hängt stark von der Zusammensetzung der Proben ab. Daher soll zunächst untersucht werden, welche Phasen sich in binären Eisen-Palladium-Legierungen einstellen und wie diese sich bei einer anschließenden Glühbehandlung verhalten, bevor der Einfluss der Zugabe von Kupfer ermittelt wird. Anschließend wird die Stabilität der Kristallstruktur des binären Systems gegenüber mechanischer Verformung analysiert.

\subsubsection{Binäre Phasen bei Zimmertemperatur}

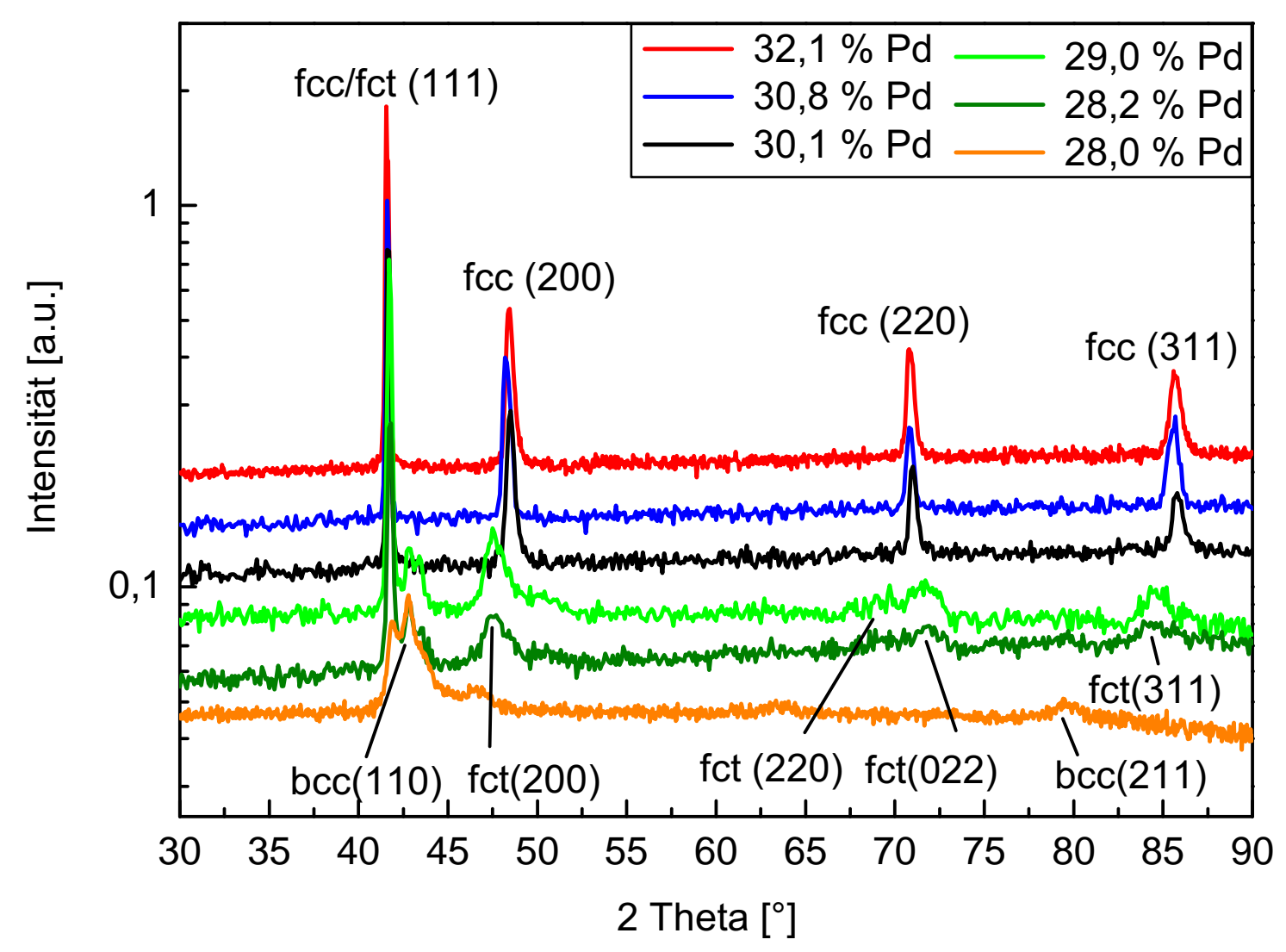

Abbildung 4.7: Anhand der Röntgendiffraktogramme binärer Proben ist zu erkennen, dass nur bei einem Palladiumgehalt zwischen $28 \%$ und $30 \%$ die martensitische fct-Phase bei Zimmertemperatur auftritt.

Der Zusammenhang zwischen der Zusammensetzung von Einkristallen und der Phase, die sich bei Zimmertemperatur einstellt, ist aus Arbeiten von Sugiyama et al. [81] und Cui et al. [6] bekannt. Matsui et al. zeigten jedoch, dass diese Ergebnisse nicht ohne Weiteres auf polykristalline Proben übertragen werden können [1] und dass die Phasen- 
bildung noch von weiteren Parametern wie beispielsweise der Abschreckgeschwindigkeit abhängt. Daher wurde zunächst überprüft, bei welchen Zusammensetzungen sich in Eisen-Palladium-Splats die gewünschten Phasen Martensit und Austenit einstellen.

In Abbildung 4.7 ist zu erkennen, dass bei einem Palladiumgehalt von $28 \%$ im Wesentlichen die unerwünschte bcc-Phase auftritt. Die breiten Peaks weisen auf eine geringe Qualität der Kristallstruktur hin. Bei einem Palladiumgehalt von $30 \%$ oder mehr hingegen ist die austenitische fcc-Phase stabil und die Kristallstruktur hat eine deutlich bessere Qualität. Nur in einem schmalen Intervall zwischen $28 \%$ und $30 \%$ Palladium kann, neben einem geringen Anteil an bcc-Phase, die martensitische fct-Phase bei Zimmertemperatur gewonnen werden. Beim Abkühlen auf Stickstofftemperatur transformieren diese Proben jedoch irreversibel in die bcc-Phase.

Die Position der fcc/fct(111)-Peaks verschiebt sich mit zunehmendem Palladiumgehalt geringfügig nach links. Das bedeutet, je mehr Palladium die Probe enthält, desto größer ist der Abstand der (111)-Ebenen und damit in erster Näherung auch das Volumen der Elementarzelle. Aus den Peakpositionen in Abbildung 4.7 können Volumina zwischen $52,3 \AA^{3}$ in der tetragonal verzerrten Probe mit 28,2\% Palladium und 53,2 $\AA^{3}$ für einen Palladiumgehalt von 32,1\% abgeschätzt werden. Daraus errechnet sich eine Gitterkonstante der kubischen Zelle von $a_{f c c}=3,76 \AA$. Eine Bestimmung der tetragonalen Gitterparameter der martensitischen Proben aus diesen Messungen ist wegen der starken Verbreiterung der Peaks höherer Ordnung nicht möglich.

\subsubsection{Einfluss der Glühbehandlung}

Wie in Kapitel 3 beschrieben, wurden einige Proben einer Glühbehandlung unterzogen, um ihre Mikrostruktur und Kristallstruktur zu verbessern. Nach dem Tempern werden die Proben mit einer wesentlich geringeren Geschwindigkeit abgekühlt als beim Splatquenching. Dadurch kann, wie in Abschnitt 2.7 erwähnt, die Phasenbildung beeinflusst werden.

Zunächst wurden Proben mit einem Palladiumgehalt von $29 \%$ untersucht, die bei Zimmertemperatur martensitisch sind. Diese Proben neigen, unabhängig von Temperatur und Dauer der Glühbehandlung, zur Bildung von Eisenausscheidungen. Diese treten gelegentlich auch bei relativ hohen Abkühlraten durch Abschrecken in Wasser auf. Proben, die nach der Glühbehandlung keine Ausscheidungen aufweisen, liegen zumindest zum Teil in der bct-Phase vor (siehe Abbildung 4.8). Der Anteil der bct-Phase steigt dabei mit der Dauer und Temperatur der Glühbehandlung.

Des Weiteren ist festzuhalten, dass Intensität und Schärfe der Röntgenreflexe bei kurzer Glühdauer deutlich geringer sind als bei unbehandelten oder lange geglühten Proben. Die Position des fct(111)-Peaks verändert sich bei kurzem Glühen nicht, der fct(200)Peak wandert jedoch zu kleineren Winkeln, d.h. die tetragonale Verzerrung steigt.

Proben mit einem Palladiumgehalt von $30 \%$ neigen deutlich weniger zum Entmischen. Die in Abbildung 4.9 gezeigten Proben wurden alle für eine Stunde bei $850{ }^{\circ} \mathrm{C}$ geglüht. Bei hinreichend hohen Abkühlraten, die nicht nur durch Abschrecken in Wasser, sondern auch durch Abkühlen an Luft erreicht werden, treten nur in Ausnahmefällen Ausscheidungen auf. Lediglich bei sehr geringer Abkühlgeschwindigkeit, wenn die Probe im 


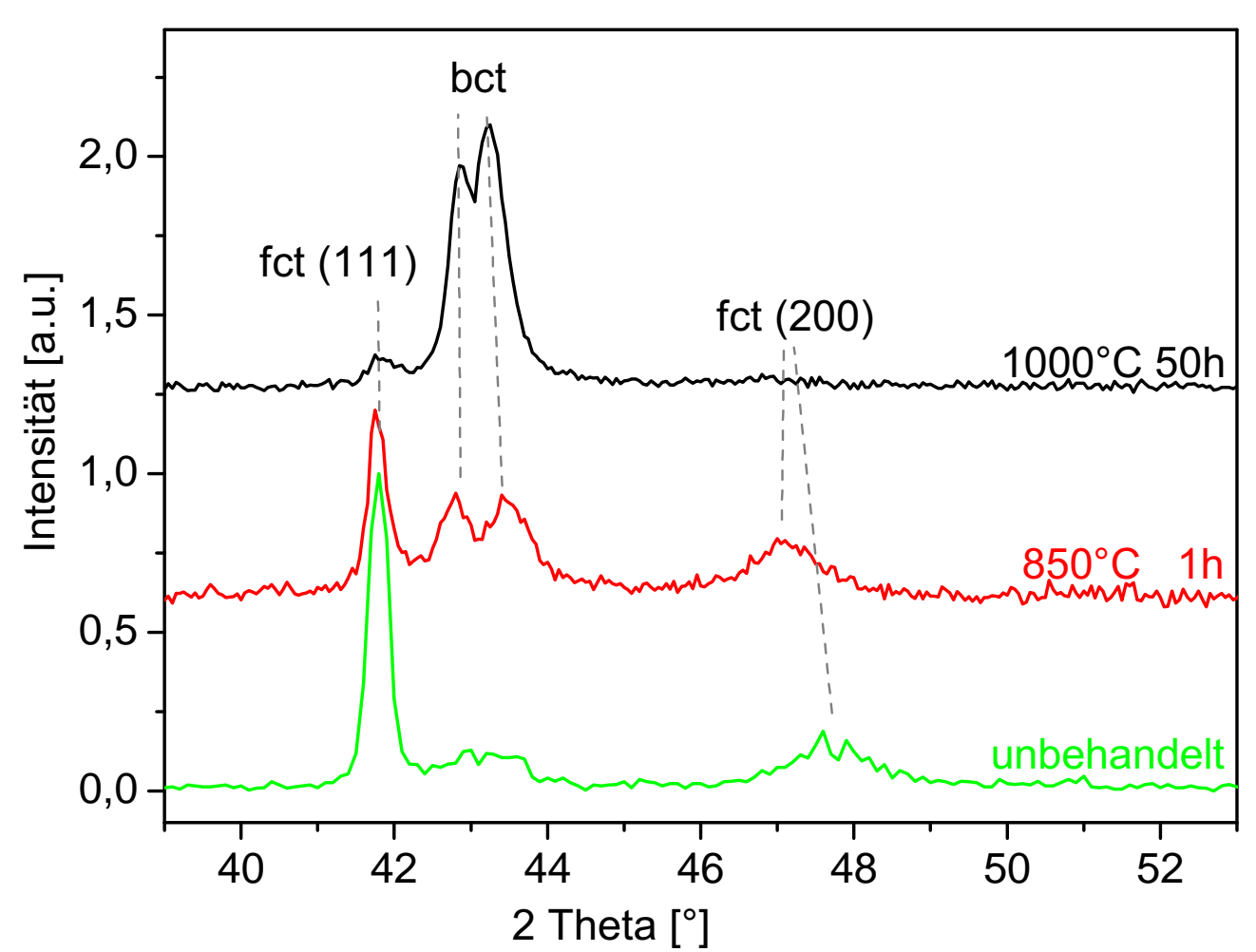

Abbildung 4.8: Sofern keine Ausscheidungen auftreten, steigt in Proben mit einem Palladiumgehalt von $29 \%$ der Anteil der bct-Phase mit der Dauer und Temperatur der Glühbehandlung.

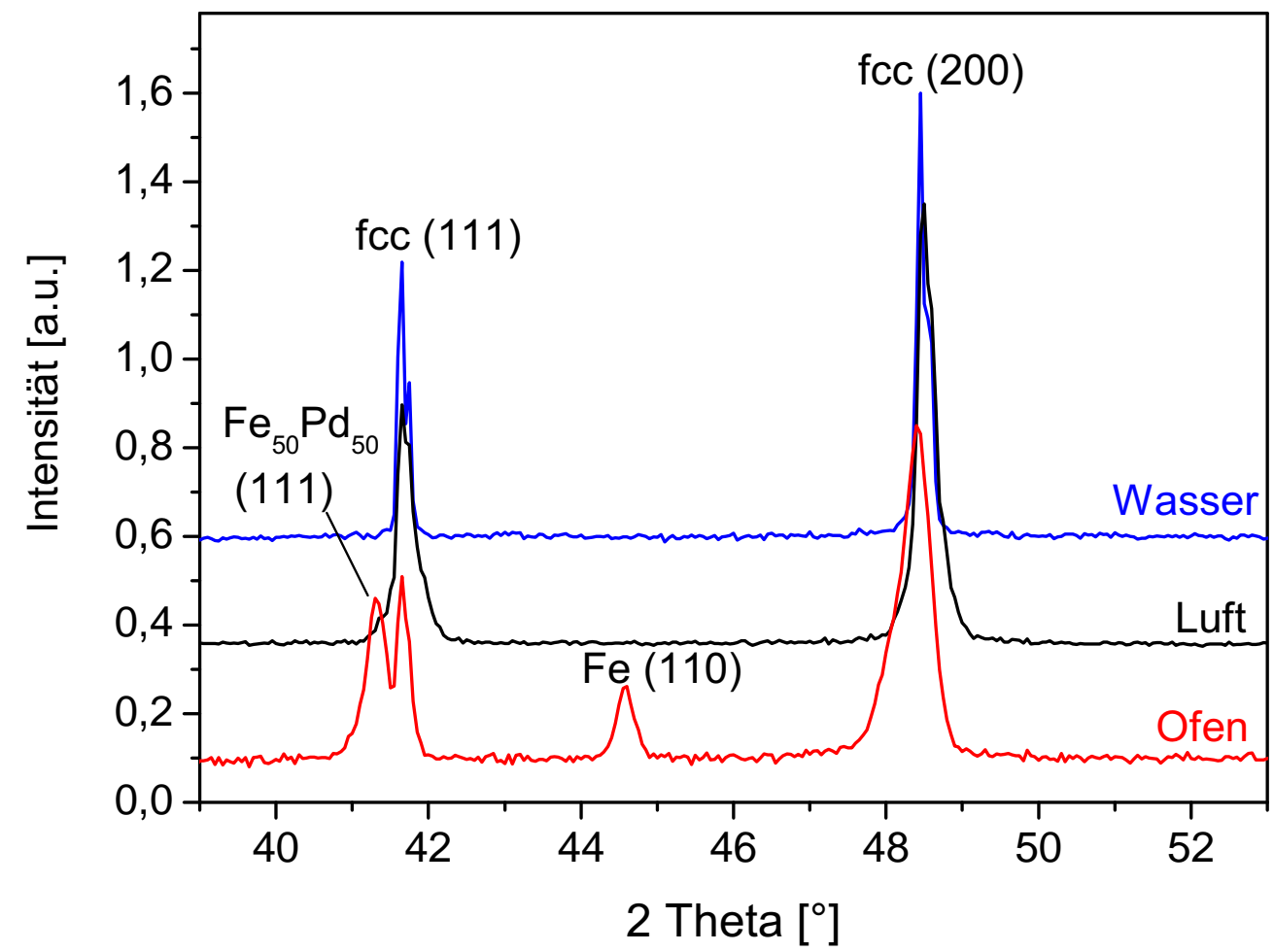

Abbildung 4.9: Proben mit $30 \%$ Palladium sind weitestgehend stabil gegen Entmischen. Nur bei sehr niedrigen Abkühlraten (beim Abkühlen im Ofen) entstehen Ausscheidungen. 
Ofen verbleibt, kommt es zu signifikanter Separation in die geordnete $\mathrm{Fe}_{50} \mathrm{Pd}_{50}$-Phase und $\alpha$-Eisen. Die Bildung von Ausscheidungen führt zu einer deutlichen Peakverbreiterung. Allgemein sind die Röntgenreflexe umso schärfer, je schneller die Probe abgekühlt wurde.

\subsubsection{Zugabe von Kupfer}

Für die meisten Anwendungen der Legierung Eisen-Palladium sind die austenitische fcc-Phase oder die martensitische fct-Phase nötig, die sich reversibel ineinander umwandeln. Im binären System lässt sich die Umwandlungstemperatur durch eine Reduktion des Palladiumgehalts erhöhen [6]. Die Ergebnisse in Abschnitt 4.2.2 zeigen jedoch, dass das System dabei zur Bildung der bcc/bct-Phase und zum Entmischen neigt. Um die Stabilität der fcc/fct-Phase gegen Entmischen und gegen Bildung der bcc-Phase auch bei geringerem Palladiumgehalt zu erhöhen, wurden kleine Mengen Kupfer zulegiert. Basierend auf Untersuchungen von Hamann et al. [25], wurden drei besonders vielversprechende Zusammensetzungen ausgewählt. Zunächst wurde bei einem Eisengehalt von $70 \%$, der sich im binären System als besonders stabil erwiesen hat, ein Teil des Palladiums durch 6,2\% Kupfer ersetzt. In einer anderen Probe wurde bei einem Eisengehalt von $71,2 \%$, der im binären System nicht mehr stabil ist, ein geringerer Teil des Palladiums durch 1,0\% Kupfer ersetzt. Des Weiteren wurde in einer Probe mit einem Palladiumgehalt von 29,3\%, der im binären System gerade noch stabil ist, ein Teil des Eisens durch 2,3\% Kupfer ersetzt. Die Proben wurden jeweils für eine Stunde bei $850^{\circ} \mathrm{C}$ geglüht und anschließend entweder in Wasser abgeschreckt, oder sie kühlten an Luft bzw. im Ofen ab. In den folgenden Abschnitten wird gezeigt, wie sich die Glühbehandlung und die verschiedenen Abkühlgeschwindigkeiten auf die Kristallstruktur auswirken und inwiefern die Substitution von Eisen oder Palladium durch Kupfer die Stabilität der fcc-Phase beeinflusst.

Bei einer Zusammensetzung von $\mathrm{Fe}_{70,0} \mathrm{Pd}_{23,8} \mathrm{Cu}_{6,2}$ wird die Phasenstabilität nicht erhöht. Die Diffraktogramme in Abbildung 4.10 zeigen, dass im Gegensatz zum binären Material die Qualität der Kristallstruktur umso schlechter ist, je schneller die Proben abgeschreckt wurden. Das Diffraktogramm der nicht geglühten Probe zeigt im Wesentlichen große Mengen der unerwünschten bcc- bzw. bct-Phase sowie einen Peak bei etwa $42,2^{\circ}$, der nicht eindeutig der fcc- oder bcc-Struktur zugeordnet werden kann. Beim Tempern wandert der Peak zu 41,9 $9^{\circ}$ und kann als (111)-Peak der fcc- oder fct-Phase identifiziert werden. Die Probe wandelt sich also größtenteils in die fcc- bzw. fct-Phase um, bei hohen Abkühlraten bleiben jedoch geringe Mengen der bcc-Phase bestehen. Da die in Wasser abgeschreckte Probe keinen (200)-Peak zeigt, kann nicht zwischen der fcc- und der fct-Phase unterschieden werden. Bei moderaten Abkühlraten an Luft oder im Ofen treten massive Ausscheidungen von $\mathrm{Fe}_{8} \mathrm{Cu}_{2}$ und $\mathrm{Fe}_{50} \mathrm{Pd}_{50}$ auf. Kühlt die Probe im Ofen ab, so tritt im Diffraktogramm ein zusätzlicher Peak bei $46^{\circ}$ auf, der Ausscheidungen von Palladium zugeordnet werden kann.

In Proben der Zusammensetzung $\mathrm{Fe}_{71,2} \mathrm{Pd}_{27,8} \mathrm{Cu}_{1,0}$ ist die fcc-Phase gegenüber binären Proben mit gleichem Eisen- bzw. Palladiumgehalt stabilisiert. Wie Abbildung 4.11 zeigt, liegen die Proben im unbehandelten Zustand in der fcc- oder fct-Phase vor. Da weder ein deutlicher fct(200)-Peak noch ein fcc(200)-Peak zu erkennen ist, können die beiden Strukturen nicht unterschieden werden. Möglicherweise ist der fct(200)-Peak 


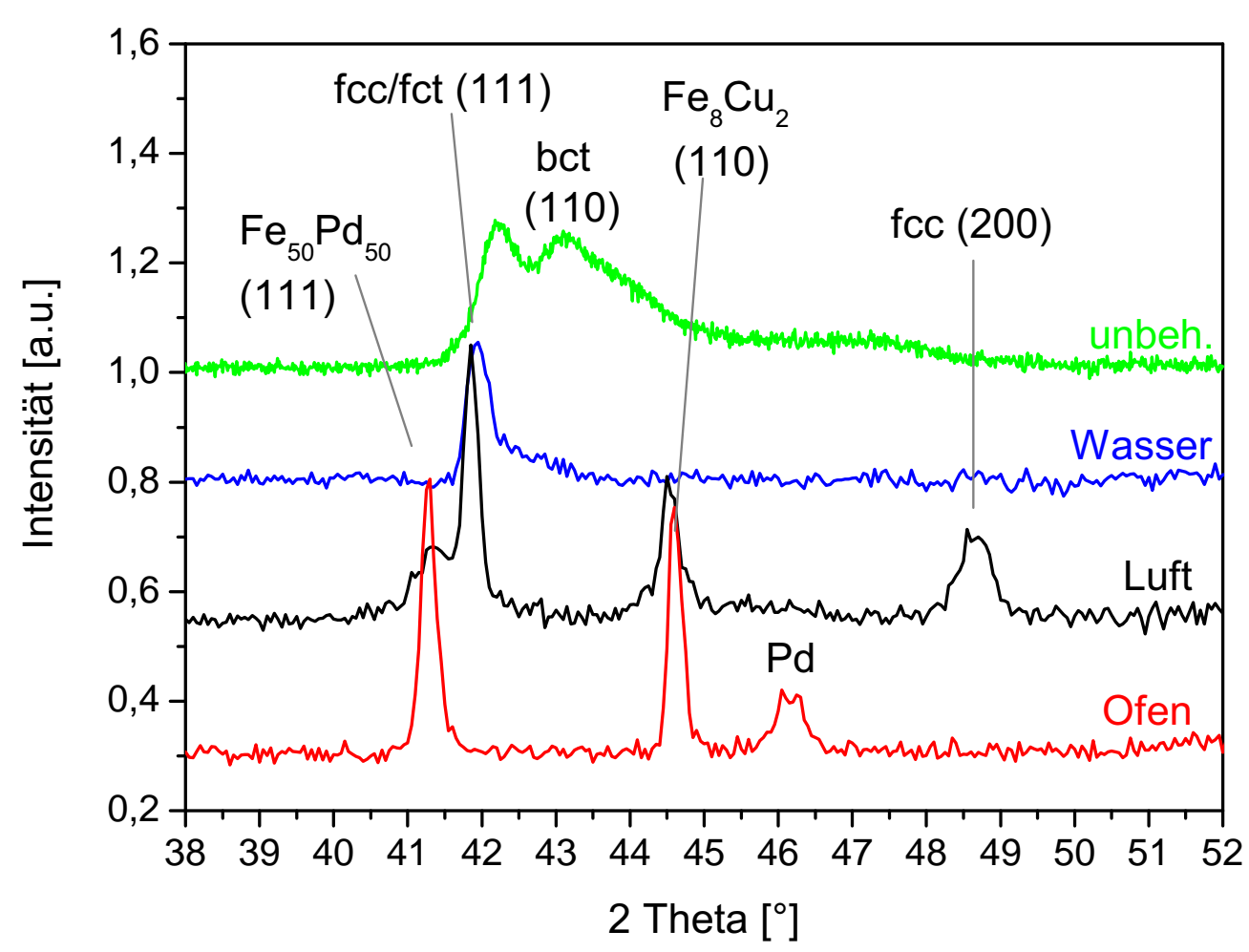

Abbildung 4.10: In Proben mit einer Zusammensetzung von $\mathrm{Fe}_{70,0} \mathrm{Pd}_{23,8} \mathrm{Cu}_{6,2}$ ist die fcc-Phase bei allen Abkühlraten (durch Abschrecken im URQ (ohne Glühbehandlung) oder in Wasser bzw. Abkühlen an Luft oder im Ofen) destabilisiert. Es treten Peaks der bct-Phase sowie verschiedene Ausscheidungen auf.

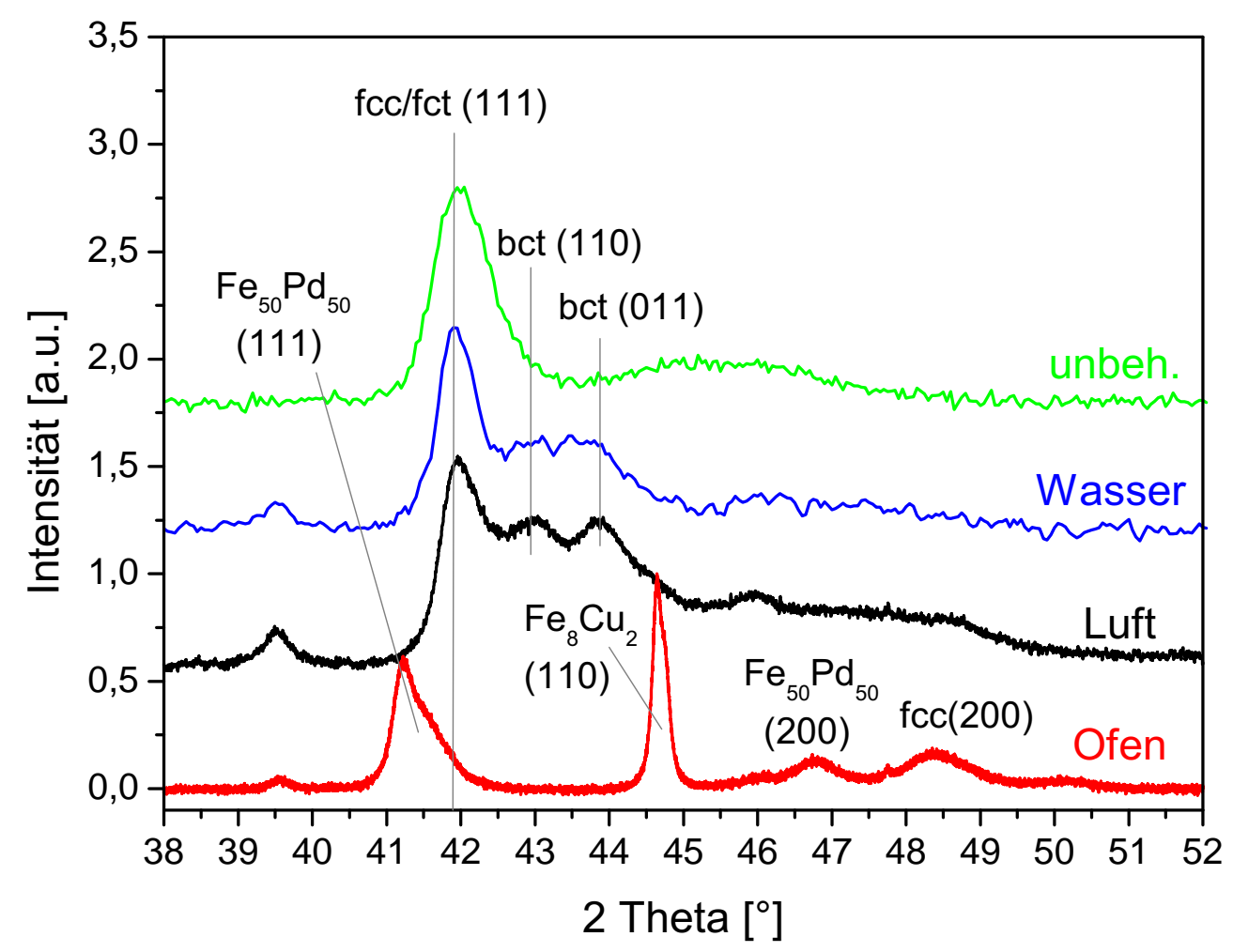

Abbildung 4.11: In Proben mit einer Zusammensetzung von $\mathrm{Fe}_{71,2} \mathrm{Pd}_{27,8} \mathrm{Cu}_{1,0}$ treten neben der fcc-Phase Peaks der bct-Phase sowie verschiedene Ausscheidungen auf. 


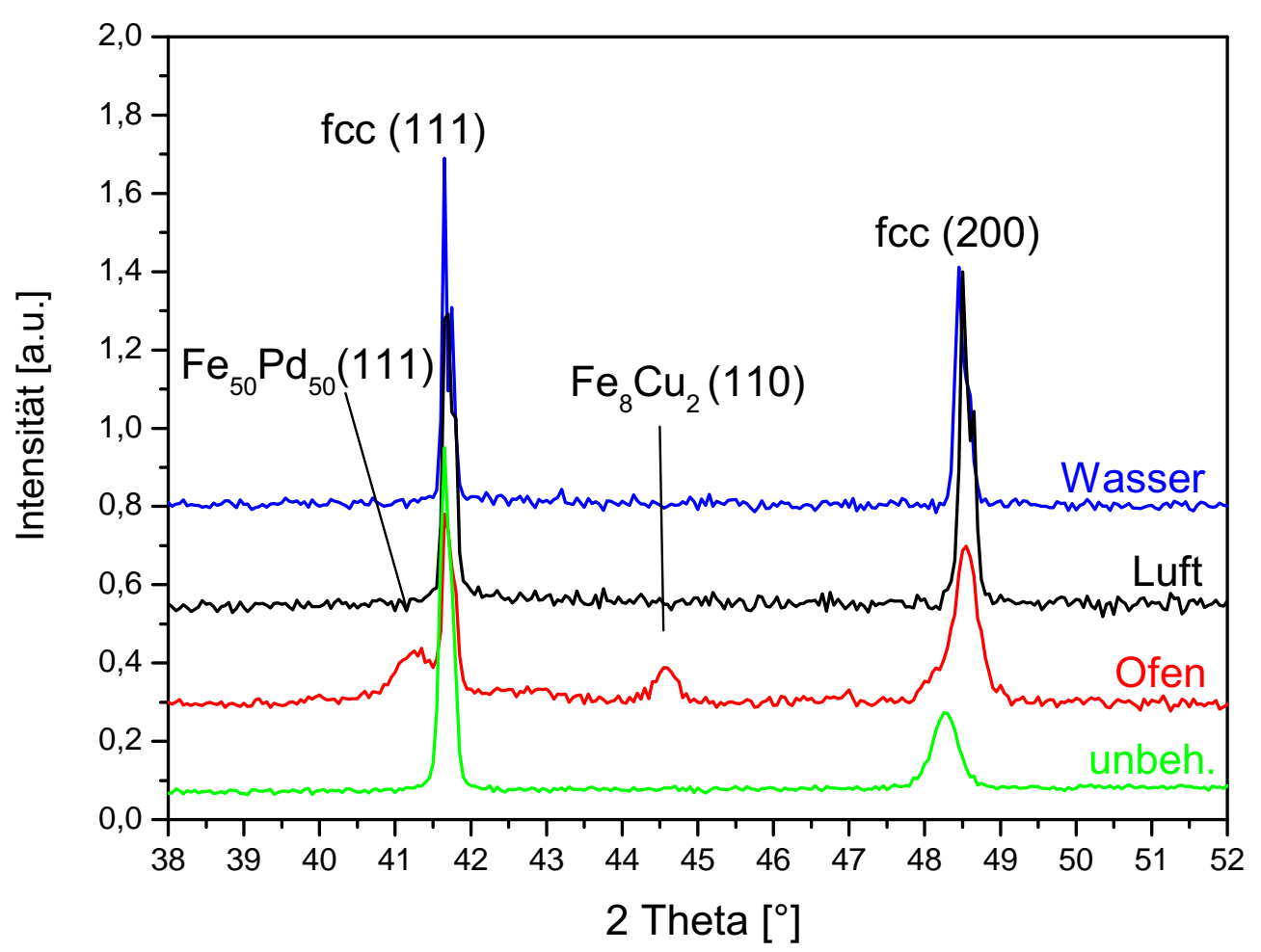

Abbildung 4.12: Proben der Zusammensetzung $\mathrm{Fe}_{68,4} \mathrm{Pd}_{29,3} \mathrm{Cu}_{2,3}$ liegen in der gewünschten fcc-Phase vor. Nur bei sehr langsamem Abkühlen im Ofen bilden sich Ausscheidungen von $\mathrm{Fe}_{50} \mathrm{Pd}_{50}$ und $\mathrm{Fe}_{8} \mathrm{Cu}_{2}$.

unter dem breiten Maximum zwischen $43,5^{\circ}$ und $48^{\circ}$ verborgen. Generell deutet die Breite des (111)-Reflexes auf eine schlechte Qualität der Kristallstruktur hin. Nach dem Glühen enthält die Probe größere Anteile in der bct-Phase sowie Ausscheidungen, die keiner der bisher beobachteten Phasen zugeordnet werden können. Beim langsamen Abkühlen im Ofen entmischt das Material in die $\mathrm{Fe}_{50} \mathrm{Pd}_{50}$-Phase und $\mathrm{Fe}_{8} \mathrm{Cu}_{2}$. Es bleibt jedoch auch ein Teil der fcc-Phase bestehen.

Eine Zusammensetzung von $\mathrm{Fe}_{68,4} \mathrm{Pd}_{29,3} \mathrm{Cu}_{2,3}$ führt zu einer deutlichen Erhöhung der Stabilität der fcc-Phase (siehe Abbildung 4.12). Schon direkt nach dem Splatquenching liegt die Probe in der gewünschten fcc-Phase ohne bcc-Anteile vor und die schmalen Peaks weisen auf eine gute Qualität der Kristallstruktur hin. Der fcc(111)-Peak liegt bei $41,65^{\circ}$, was einem Gitterparameter von $a=3,75 \AA$ entspricht. Der (200)-Peak der unbehandelten Probe ist um $0,25^{\circ}$ zu kleineren Winkeln verschoben, was auf eine leichte tetragonale Verzerrung von etwa $0,5 \%$ hindeutet. Beim Glühen bilden sich keine Ausscheidungen oder unerwünschten Phasen. Lediglich bei sehr geringen Abkühlraten kommt es zu geringfügiger Ausscheidung von $\mathrm{Fe}_{50} \mathrm{Pd}_{50}$ und $\mathrm{Fe}_{8} \mathrm{Cu}_{2}$.

\subsubsection{Mechanische Stabilität der Kristallstruktur}

In den Abschnitten 4.2.2 und 4.2.3 wurde die thermodynamische und kinetische Stabilität der Phasen gegen das Entmischen untersucht. In diesem Abschnitt soll nun die mechanische Stabilität im Fokus stehen. Eine $\mathrm{Fe}_{70} \mathrm{Pd}_{30}$-Probe, die direkt nach dem Splatquenching in der austenitischen fcc-Phase vorliegt, wurde durch manuelles Häm- 


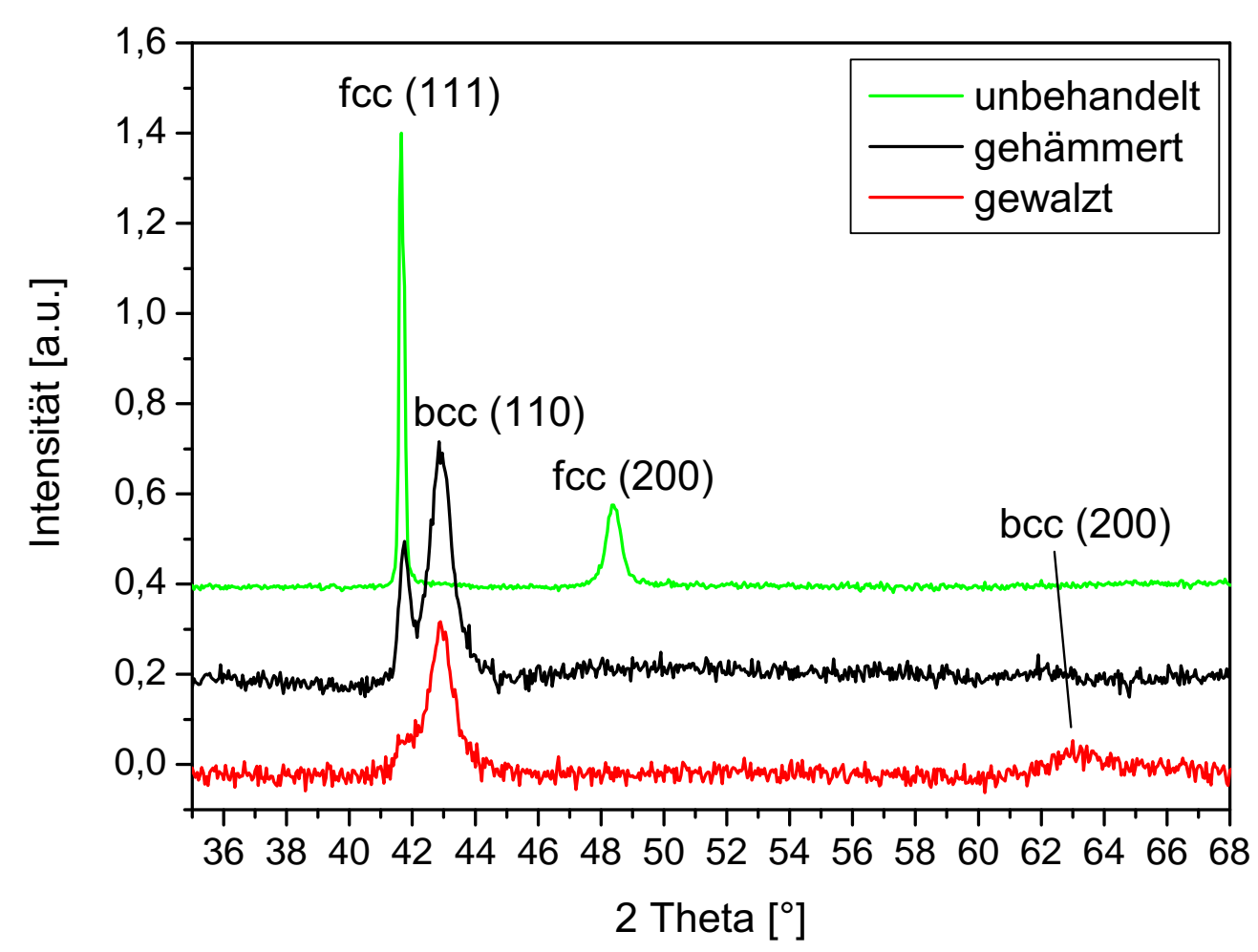

Abbildung 4.13: Bei starker mechanischer Verformung transformiert die Kristallstruktur von fcc nach bcc.

mern massiv mechanisch verformt. Anschließende Röntgenmessungen in Abbildung 4.13 zeigen, dass dabei ein erheblicher Anteil der Kristallstruktur in die bcc-Phase transformiert. Eine noch stärkere Verformung einer $\mathrm{Fe}_{70} \mathrm{Pd}_{30}$-Probe durch Kaltwalzen, bis die Probendicke etwa auf die Hälfte reduziert war, führt zu einer vollständigen Umwandlung in die bcc-Phase. Anhand der Peakverbreiterung ist zu erkennen, dass die Qualität der Kristallstruktur dabei erheblich abnimmt.

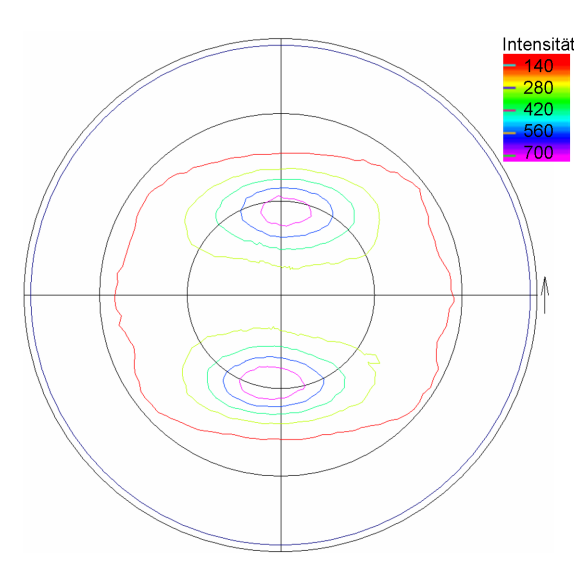

(a) gewalzt

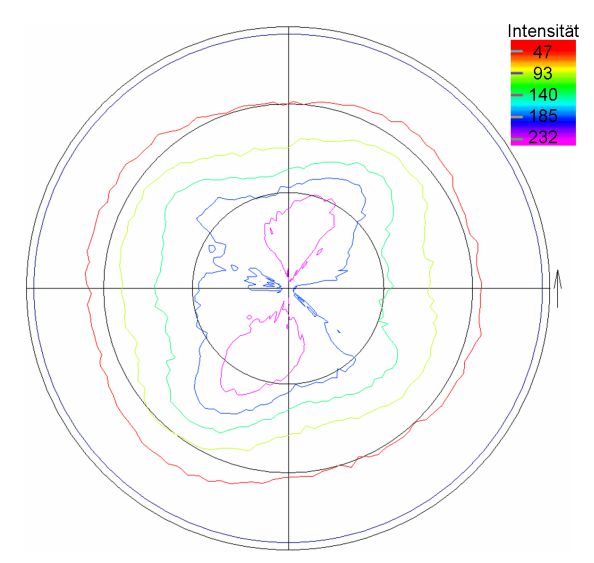

(b) angelassen

Abbildung 4.14: Diese (110)-Polfiguren zeigen, dass die Proben nach dem Walzen eine (110)-Walztextur haben, die beim Anlassen weitestgehend verschwindet. Die Walzrichtung weist nach oben. 
Wie in Abschnitt 4.1 beschrieben wurde, sind die unbehandelten Proben nicht texturiert. Durch das Walzen erhalten sie eine typische Textur, die sogenannte Walztextur, deren Polfigur in Abbildung 4.14 gezeigt ist. Die Walzrichtung weist in dieser Abbildung nach oben. Die beiden bcc(110)-Pole liegen erwartungsgemäß $60^{\circ}$ auseinander. Wird die Probe nach dem Walzen für 15 Minuten bei $900^{\circ} \mathrm{C}$ angelassen, verschwindet die Textur weitestgehend, und die Probe transformiert wieder in die austenitische fcc-Struktur zurück.

Auch durch elastische Verformung kann die Kristallstruktur der Proben verändert werden. Abbildung 4.15 zeigt Röntgendiffraktogramme einer Probe der Zusammensetzung $\mathrm{Fe}_{70} \mathrm{Pd}_{30}$, die uniaxial gezogen wurde. Im ungedehnten Zustand liegt diese Probe in der fcc-Struktur vor. Der fcc(111)- und der fcc(200)-Peak sind deutlich zu erkennen. Im gedehnten Zustand verschwindet der fcc(200)-Peak, der fcc/fct(111)-Peak hingegen bleibt bestehen und ändert seine Position nicht. Dies ist ein deutliches Anzeichen für eine spannungsinduzierte Transformation in fein verzwillingten Martensit mit geringer Kohärenzlänge in (200)-Richtung. Beim Entlasten taucht der fcc(200)-Peak wieder auf, wie es bei der Rücktransformation von spannungsinduziertem Martensit zu erwarten ist.

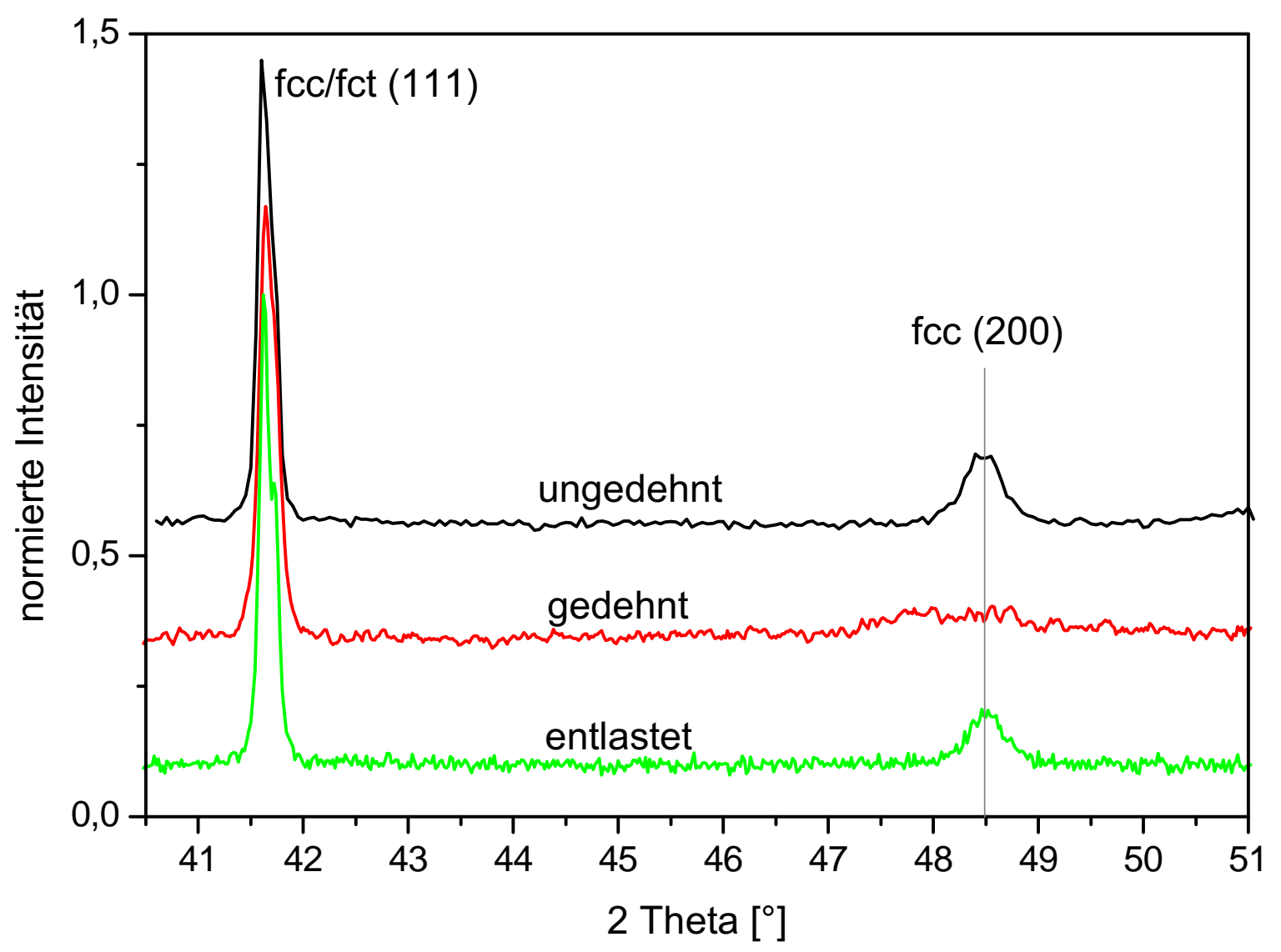

Abbildung 4.15: Beim Dehnen der austenitischen $\mathrm{Fe}_{70} \mathrm{Pd}_{30}$-Probe tritt spannungsinduzierter Martensit auf, der sich beim Entlasten zurückbildet. 


\subsection{Der martensitische Phasenübergang}

Nach der Charakterisierung der Phasen bei Zimmertemperatur soll in diesem Abschnitt nun die Temperaturabhängigkeit verschiedener Probeneigenschaften untersucht werden. Insbesondere ist die Änderung der strukturellen, magnetischen und mechanischen Eigenschaften und des elektrischen Widerstandes während des martensitischen Phasenübergangs und deren Abhängigkeit von der vorherigen Glühbehandlung von Interesse.

\subsubsection{Kristallstruktur}

Während des martensitischen Phasenübergangs in Eisen-Palladium transformiert die kubisch flächenzentrierte (fcc) austenitische Phase in die tetragonal flächenzentrierte (fct) martensitische Phase. Die Entstehung der tetragonalen Verzerrung kann mit Hilfe von Röntgendiffraktogrammen beobachtet werden. In einer kubischen Struktur sind beispielsweise alle [100]-Richtungen äquivalent, in einer tetragonalen Struktur hingegen, deren Einheitszelle über zwei lange und eine kurze Achse verfügt, haben die (100)- und (010)-Ebenen einen größeren Abstand als die (001)-Ebenen. Dies äußert sich in einer Aufspaltung der entsprechenden Röntgenreflexe. In den meisten Röntgenmessungen an transformierenden Proben, die im Rahmen dieser Arbeit durchgeführt wurden, ist eine solche Aufspaltung jedoch nicht zu beobachten. Stattdessen ist, wie bereits in Abschnitt 4.1 erwähnt, nur eine Verschiebung der Peaks zu sehen.

Ein typisches Beispiel der Peakverschiebung während des martensitischen Phasenüberganges einer $\mathrm{Fe}_{71} \mathrm{Pd}_{29}$-Probe ist in Abbildung 4.16 gezeigt. Dabei fällt auf, dass sich die Position des (111)-Peaks kaum verändert, also die Länge der Raumdiagonale der Einheitszelle etwa konstant bleibt. Des Weiteren erfolgt die Verschiebung des (200)Peaks nicht schlagartig, sondern graduell. Der fcc(200)-Peak entwickelt zunächst eine Schulter, aus welcher der fct(200)-Peak entsteht. Diese Schulter entsteht durch die Koexistenz der fcc- und der fct-Phase und zeigt den Beginn der Phasenumwandlung an, so dass die Martensitstarttemperatur $M_{s}$ auf etwa $52 \pm 5{ }^{\circ} \mathrm{C}$ abgeschätzt werden kann. Bei der Martensitendtemperatur $M_{f}=30 \pm 5{ }^{\circ} \mathrm{C}$ ist der fcc(200)-Peak verschwunden, die Umwandlung ist beendet. Weiteres Abkühlen verschiebt den fct(200)-Peak langsam zu kleineren Winkeln, die $a$-Achse wird länger und erreicht bei $10^{\circ} \mathrm{C}$ eine Länge von $3,83 \pm 0,05 \AA$. Über den Abstand der (111)-Ebenen kann indirekt auch die Länge der c-Achse zu 3,55 $\pm 0,05 \AA$ bestimmt werden. Die tetragonale Verzerrung wird also bei tiefen Temperaturen größer und erreicht einen Wert von $c / a=0,93 \pm 0,02$.

Die Qualität der Kristallstruktur ist anhand der Peakverbreiterung in dieser Messung schwer zu beurteilen. Die Breite des (111)-Peaks ändert sich beim Kühlen nicht signifikant, während der fct(200)-Peak deutlich breiter ist als der fcc(200)-Peak.

Da Proben mit einem Palladiumgehalt von $30 \%$ beim Glühen in der Regel nicht entmischen, konnte für diese Zusammensetzung neben dem Transformationsverhalten unbehandelter Splats auch der Einfluss einer Glühbehandlung auf das Transformationsverhalten untersucht werden. Abbildung 4.17 zeigt Röntgendiffraktogramme von Proben der Zusammensetzung $\mathrm{Fe}_{70} \mathrm{Pd}_{30}$, die jeweils für 15 Minuten bei Temperaturen zwischen $600{ }^{\circ} \mathrm{C}$ und $1000^{\circ} \mathrm{C}$ geglüht wurden. Anhand der bei verschiedenen Temperaturen aufgenommenen Diffraktogramme kann der martensitische Phasenübergang beobachtet 


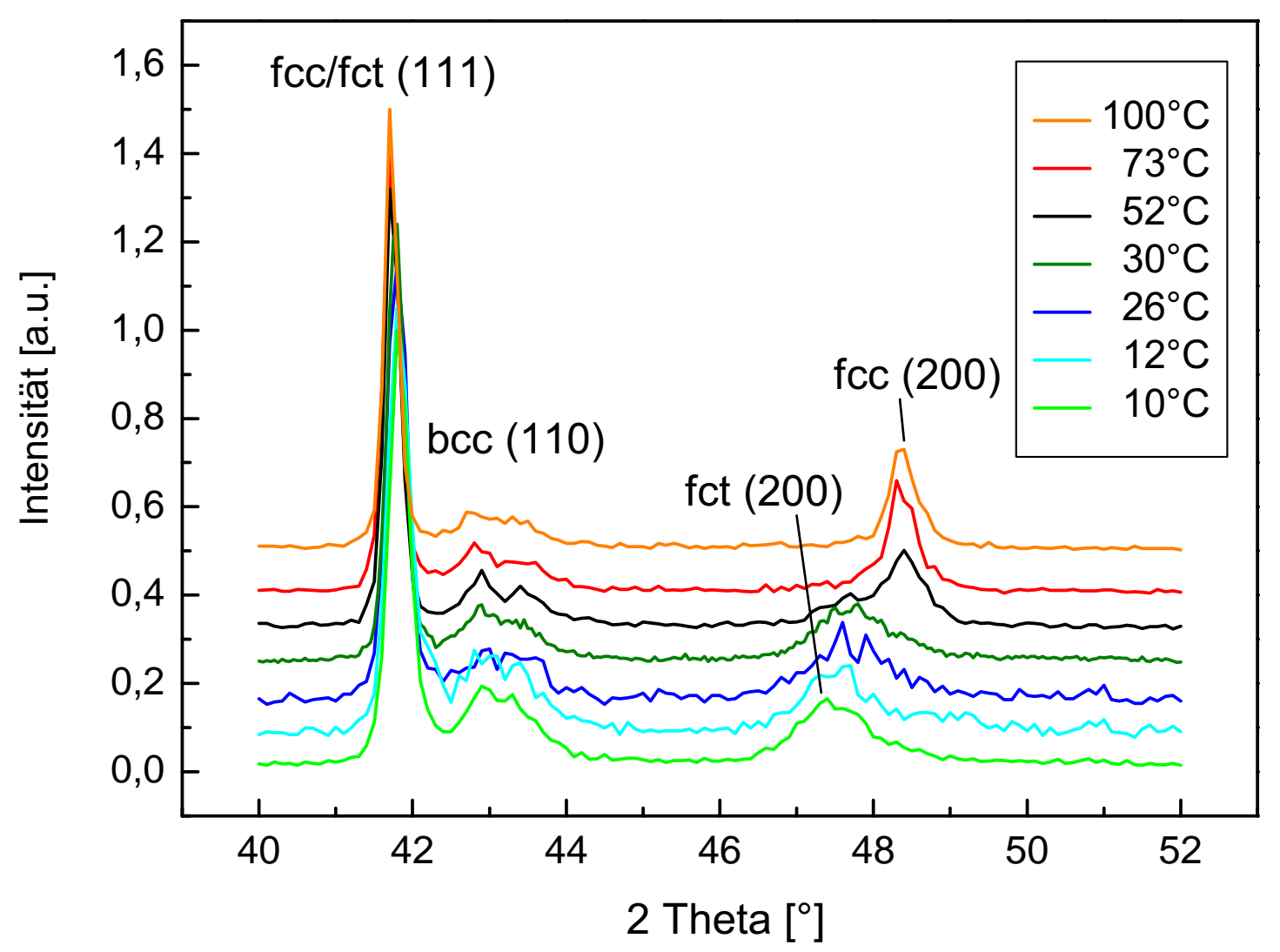

Abbildung 4.16: Dieses Röntgendiffraktogramm einer Probe der Zusammensetzung $\mathrm{Fe}_{71} \mathrm{Pd}_{29}$ zeigt, wie sich der (200)-Peak während der Umwandlung von fcc nach fct beim Kühlen zu kleineren Winkeln verschiebt. Die Position des (111)-Peaks ändert sich kaum.

werden. Es zeigt sich, dass sich auch hier nur der fcc(200)-Peak zur fct(200)-Position verschiebt und der (002)-Peak nicht auftritt. Die Verschiebung des (200)-Peaks ist bei unbehandelten Proben mit $30 \%$ Palladium deutlich geringer als bei einem Palladiumgehalt von $29 \%$, weshalb eine genaue Bestimmung der Martensitstarttemperatur $M_{s}$ schwierig ist. Daher wurde jeweils an den (200)-Peak eine Lorentz-Kurve angepasst, aus der seine Schwerpunktposition gewonnen wurde. Aus dieser kann der Gitterparameter $a$ berechnet werden, anhand dessen die Transformation verfolgt werden kann. In Abbildung 4.18 ist für alle Proben der Zusammensetzung $\mathrm{Fe}_{70} \mathrm{Pd}_{30}$ die Änderung des Gitterparameters mit der Temperatur aufgetragen. Um die Martensitstarttemperatur $M_{s}$ zu bestimmen, wurden an die Messwerte zwei Geraden angelegt. Eine Gerade kennzeichnet den Bereich, in dem sich der Gitterparameter nicht signifikant ändert. Die andere folgt dem Anstieg des Gitterparameters mit Beginn der Transformation. Der Schnittpunkt der Geraden wurde als Martensitstarttemperatur $M_{s}$ definiert.

Die Martensitendtemperatur $M_{f}$ kann mit dieser Methode nicht gewonnen werden, da sich der Gitterparameter auch nach dem Ende der Transformation noch ändert. Hier erwies es sich als zweckmäßig, die Intensität des fcc(200)-Peaks bei 48,5 (siehe Abbildung 4.17) gegen die Temperatur aufzutragen. Diese ändert sich nach Beendigung der Umwandlung nicht mehr, da der Austenit vollständig verschwunden ist (Abbildung 4.19). Die Intensität ist auf den Wert bei $20^{\circ} \mathrm{C}$ normiert, um Messungen mit und ohne 

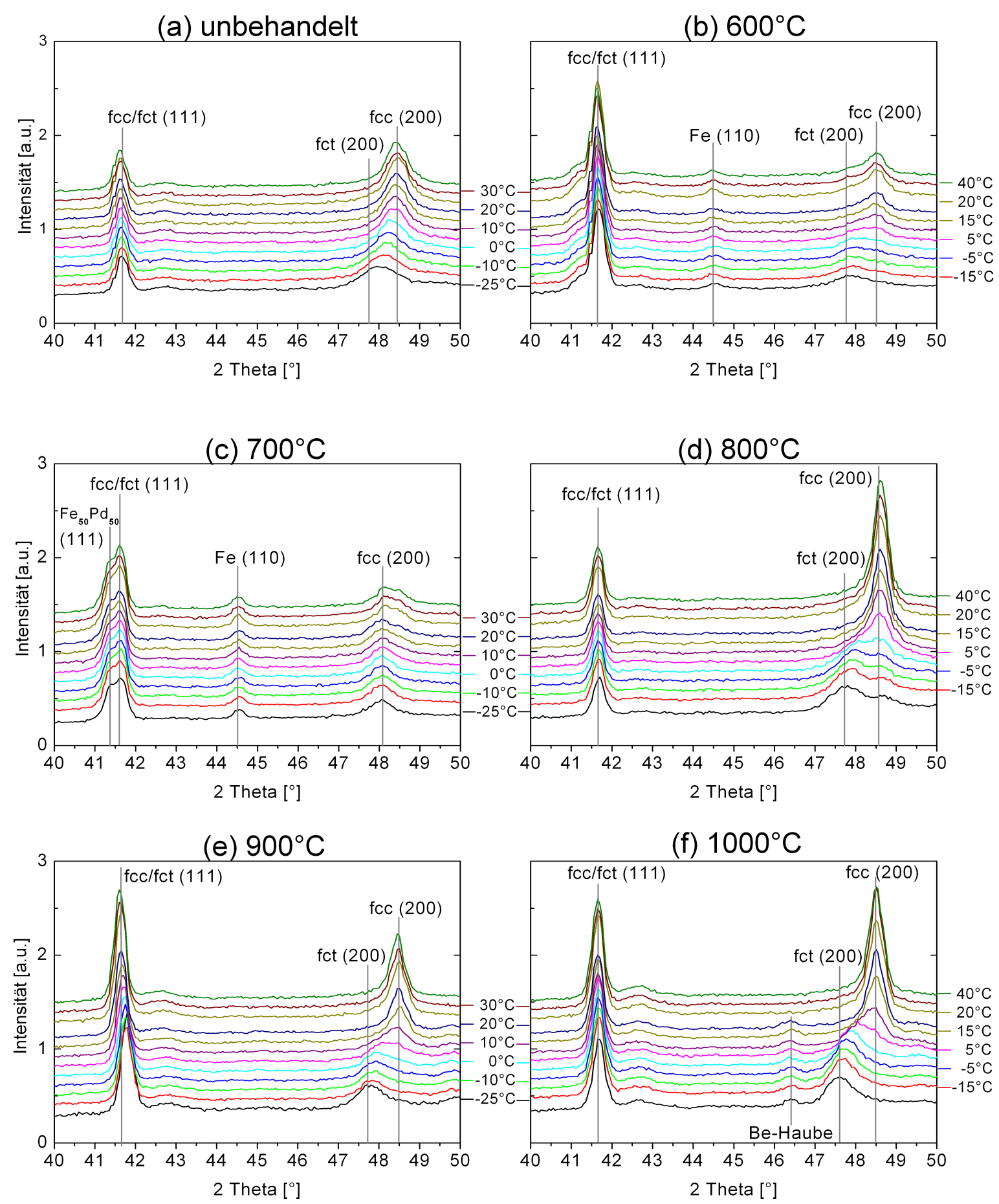

Abbildung 4.17: Diffraktogramme von $\mathrm{Fe}_{70} \mathrm{Pd}_{30}$-Proben, die bei verschiedenen Temperaturen geglüht wurden: Anhand der Verschiebung des (200)-Peaks kann der martensitische Phasenübergang verfolgt werden $(\mathrm{XRD}(\mathrm{T})$ : S.Hamann) 
Be-Haube vergleichen zu können. Dennoch flacht die Steigung bei $20^{\circ} \mathrm{C}$ bei fast allen Proben ab, weshalb diese Methode nicht zur Bestimmung von $M_{s}$ geeignet ist. An die Messwerte wurden wiederum zwei Geraden angelegt, eine an den konstanten Bereich bei niedrigen Temperaturen, eine weitere an den Abfall der Intensität. Der Schnittpunkt der Geraden liefert die Martensitendtemperatur $M_{f}$.

Es zeigt sich, dass Proben mit einem Palladiumgehalt von $30 \%$ bei deutlich tieferen Temperaturen transformieren als Proben mit einem Palladiumgehalt von 29\% und die Verschiebung des (200)-Peaks etwas weniger stark ausgeprägt ist. Die Peakverschiebung der unbehandelten Probe mit der Temperatur und die daraus berechnete Änderung des Gitterparameters $a$ ist in Abbildung 4.18(a) zu sehen. Die Länge der $a$-Achse steigt von $3,756 \pm 0,005 \AA$ bei $40{ }^{\circ} \mathrm{C}$ auf $3,788 \pm 0,005 \AA$ bei $-25^{\circ} \mathrm{C}$, während die Länge der $c$-Achse zu 3,688 $\pm 0,005 \AA$ berechnet werden kann, was ein Achsenverhältnis von $c / a=0,9735 \pm 0,0015$ ergibt. Aus der Änderung des Gitterparameters kann die Martensitstarttemperatur auf $M_{s}=12,5 \pm 2{ }^{\circ} \mathrm{C}$ abgeschätzt werden. Die Martensitendtemperatur der unbehandelten Probe ergibt sich aus dem Abfall der Intensität des fcc(200)-Peaks in Abbildung 4.19(a) zu $M_{f}=-9 \pm 2{ }^{\circ} \mathrm{C}$.

Die Diffraktogramme und die Auftragungen der Gitterparameter und Peakintensitäten in den Abbildungen 4.17 bis 4.19 für die bei verschiedenen Temperaturen geglühten Proben zeigen, dass die Glühtemperatur Kristallstruktur und Transformationsverhalten stark beeinflusst, obwohl die Proben nur für 15 Minuten geglüht und anschließend in Wasser abgeschreckt wurden. Im Folgenden sollen die Auswirkungen der verschiedenen Glühtemperaturen nacheinander betrachtet werden.

Die bei $600{ }^{\circ} \mathrm{C}$ bzw. $700{ }^{\circ} \mathrm{C}$ geglühten Proben enthalten Ausscheidungen von $\alpha$-Eisen und der geordneten $\mathrm{Fe}_{50} \mathrm{Pd}_{50}$-Phase. Der fcc bzw. fct(200)-Peak ist nicht sehr stark ausgeprägt. Die bei $700{ }^{\circ} \mathrm{C}$ geglühte Probe zeigt keine Peakverschiebung bei tiefen Temperaturen, weshalb aus Abbildung 4.18(c) keine Übergangstemperatur bestimmt werden konnte. Der Sprung des Gitterparameters bei $20^{\circ} \mathrm{C}$ fällt mit dem Entfernen der BeHaube zusammen. Da beide Werte bei der gleichen Temperatur gemessen wurden, kann ein temperaturabhängiger Effekt ausgeschlossen werden.

Die bei $600{ }^{\circ} \mathrm{C}$ geglühte Probe zeigt trotz der Eisenausscheidungen Anzeichen einer Transformation. Der (200)-Peak entwickelt eine Schulter und verschiebt sich beim Kühlen zu kleineren Winkeln. Dieses Verhalten ist auch in den Abbildungen 4.16 und 4.18(b) zu erkennen, auch wenn die Änderung des Gitterparameters geringer ausfällt als bei den heißer geglühten Proben. Der Austenit (200)-Peak verschwindet nicht vollständig, doch unterhalb von $0^{\circ} \mathrm{C}$ ändert sich seine Intensität wesentlich langsamer (siehe Abbildung 4.19(b)), weshalb die Transformation als im Wesentlichen abgeschlossen betrachtet werden kann, auch wenn noch geringe Mengen Austenit zurückbleiben.

Bei Glühtemperaturen von $800^{\circ} \mathrm{C}$ oder mehr treten keine Eisenausscheidungen mehr auf. Die fcc/fct (111)- und (200)-Peaks sind deutlich schärfer. In der bei $800^{\circ} \mathrm{C}$ geglühten Probe beginnt der Phasenübergang bei tieferen Temperaturen und erfolgt sprunghaft. Der (200)-Peak springt beim Kühlen von $5{ }^{\circ} \mathrm{C}$ auf $0{ }^{\circ} \mathrm{C}$ von der fcc- zur fct-Position. Der fcc(200)-Peak verschwindet jedoch auch in dieser Probe nicht vollständig, es bleiben also Austenitreste bestehen.

Die bei $900{ }^{\circ} \mathrm{C}$ bzw. $1000^{\circ} \mathrm{C}$ geglühten Proben verhalten sich sehr ähnlich, wobei die Intensität des fcc(200)- und des fct(200)-Peaks sowie die Änderung des Gitterparame- 


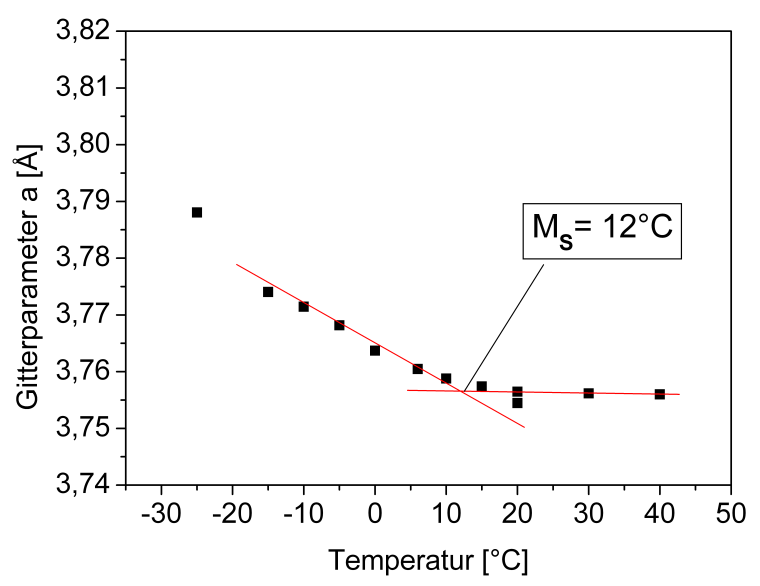

(a) unbehandelt

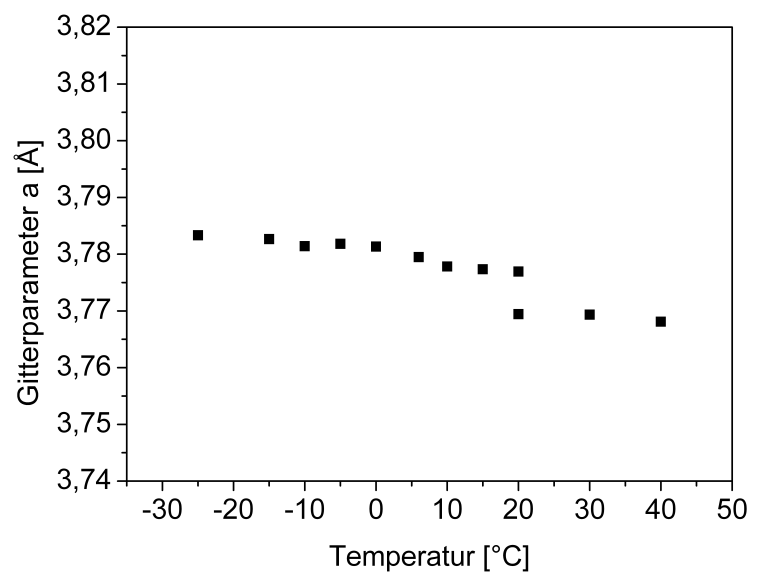

(c) $700{ }^{\circ} \mathrm{C}$

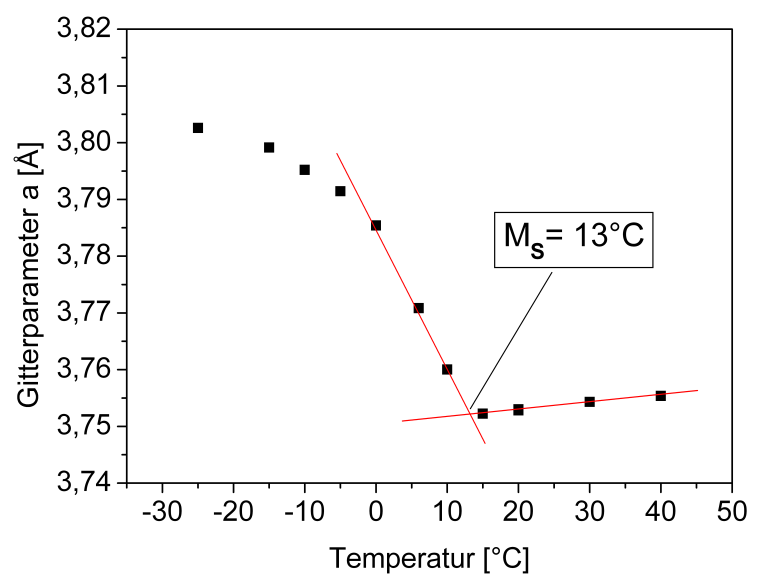

(e) $900{ }^{\circ} \mathrm{C}$

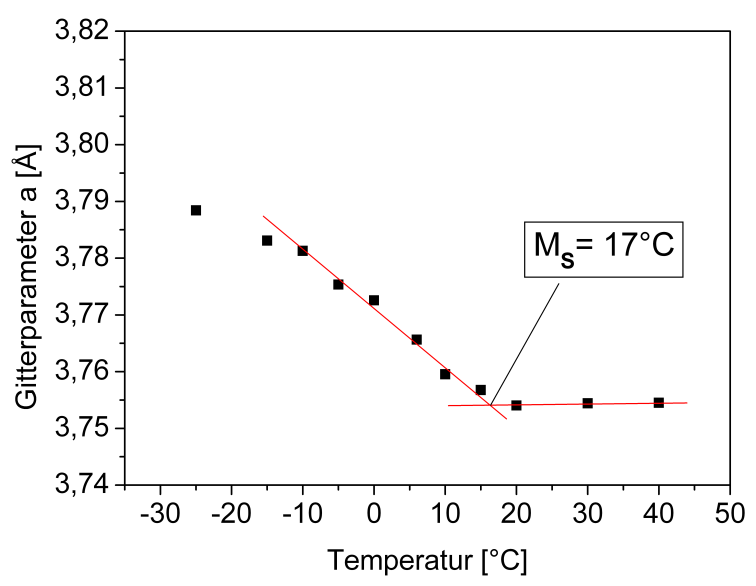

(b) $600{ }^{\circ} \mathrm{C}$

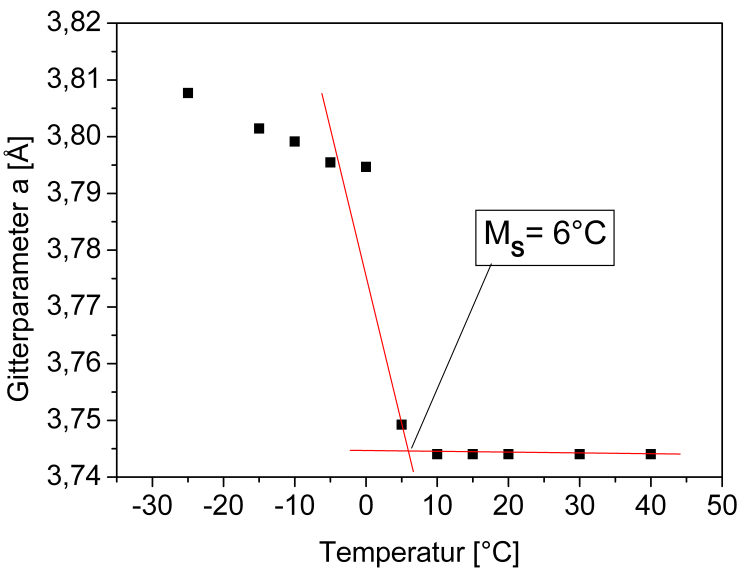

(d) $800{ }^{\circ} \mathrm{C}$

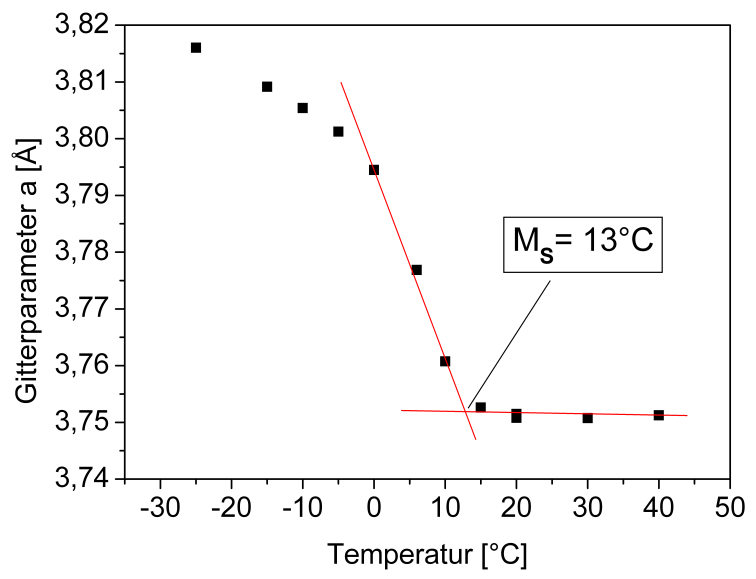

(f) $1000{ }^{\circ} \mathrm{C}$

Abbildung 4.18: Aus dem Schnittpunkt der Geraden, der das Einsetzen der Gitterverzerrung markiert, kann die Martensitstarttemperatur $M_{s}$ bestimmt werden. 


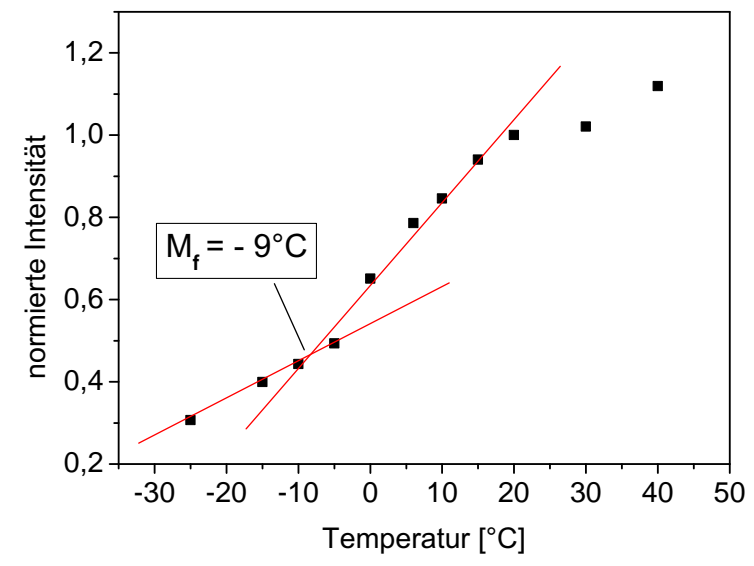

(a) unbehandelt

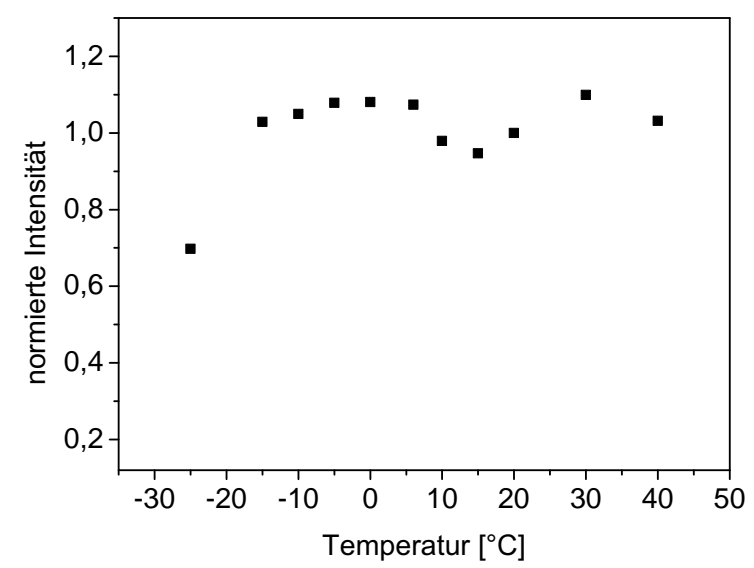

(c) $700{ }^{\circ} \mathrm{C}$

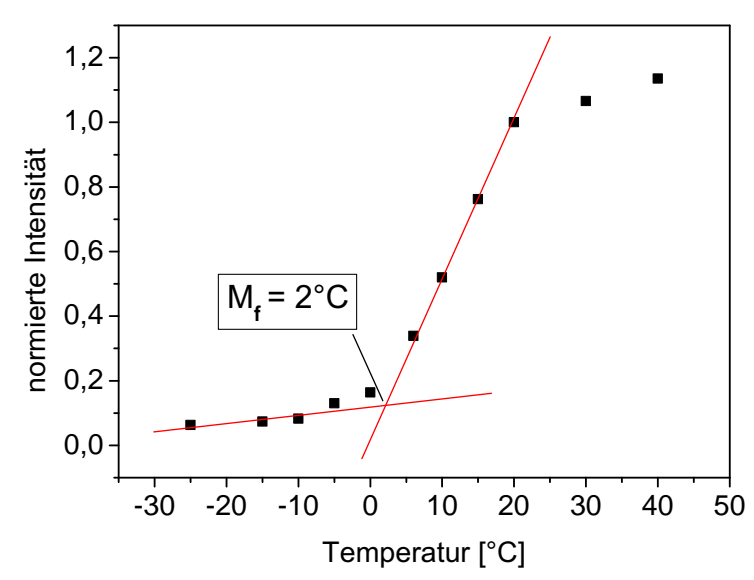

(e) $900{ }^{\circ} \mathrm{C}$

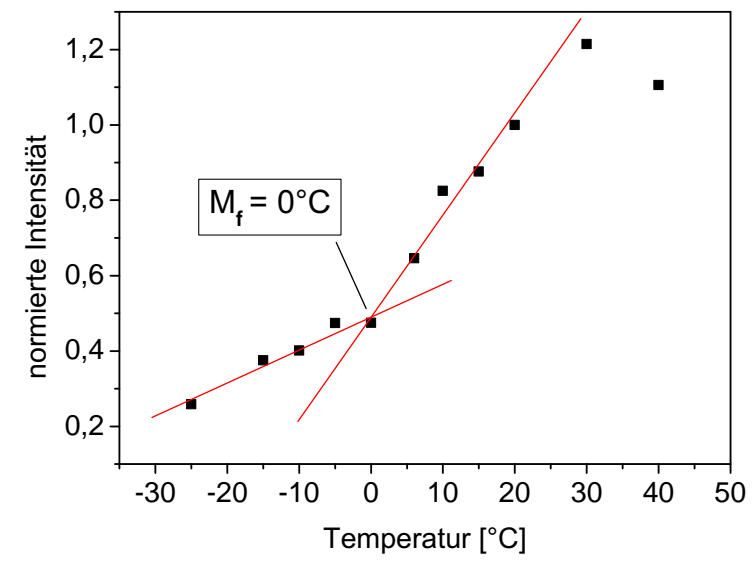

(b) $600{ }^{\circ} \mathrm{C}$

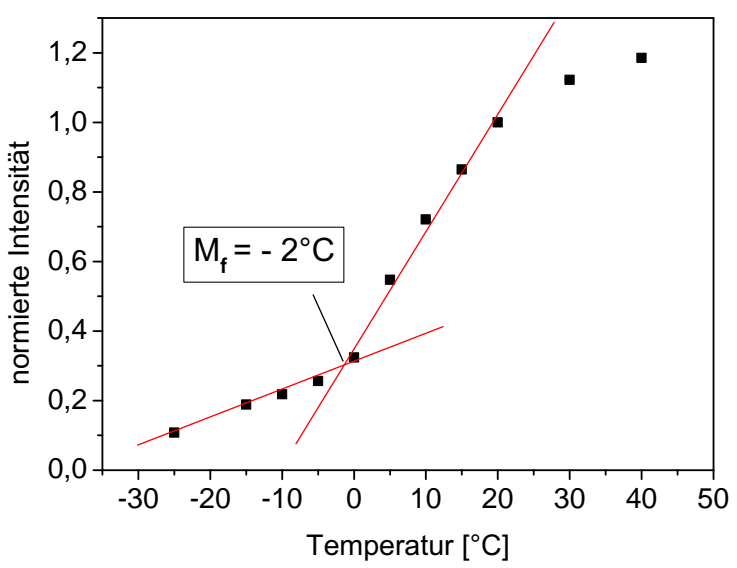

(d) $800^{\circ} \mathrm{C}$

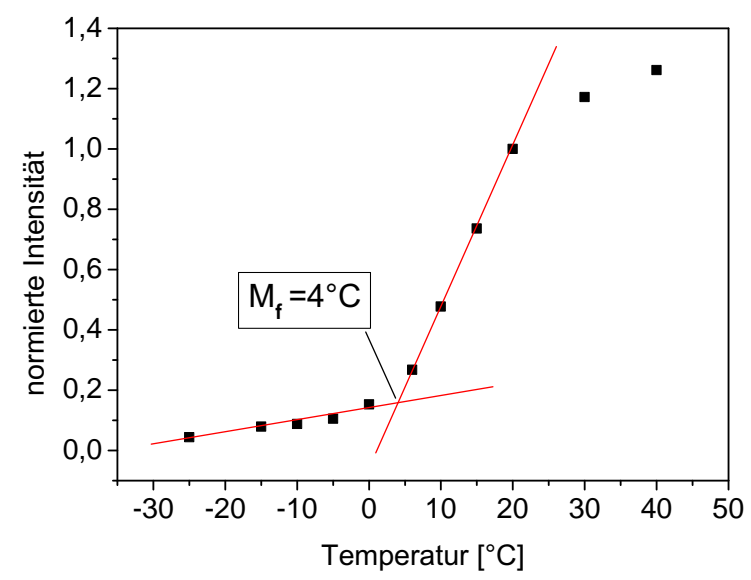

(f) $1000^{\circ} \mathrm{C}$

Abbildung 4.19: Während der Phasenumwandlung fällt die Intensität des fcc(200)Peaks aus den Röntgenmessungen in Abbildung 4.17 stark ab. Aus dem Abknicken der Steigung bei tiefen Temperaturen kann die Martensitendtemperatur bestimmt werden. Das Abknicken bei hohen Temperaturen wird durch das Entfernen der Berillium-Haube verursacht. 
ters bei letzterer am größten ist. Die $a$-Achse wächst von $3,816 \pm 0,005 \AA$ bei $40^{\circ} \mathrm{C}$ bis $3,816 \pm 0,005 \AA$ bei $-25^{\circ} \mathrm{C}$. Die Länge der $c$-Achse hingegen sinkt auf $3,619 \pm 0,005 \AA$, womit sich ein Achsenverhältnis von $c / a=0,948 \pm 0,002$ ergibt. Die Änderung des Gitterparameters erfolgt zwar nicht sprunghaft, wie in der bei $800^{\circ} \mathrm{C}$ geglühten Probe, aber in einem engen Temperaturintervall, so dass $M_{s}$ und $M_{f}$ nahe beieinanderliegen. In keiner der beiden Proben bleiben signifikante Austenitreste zurück.

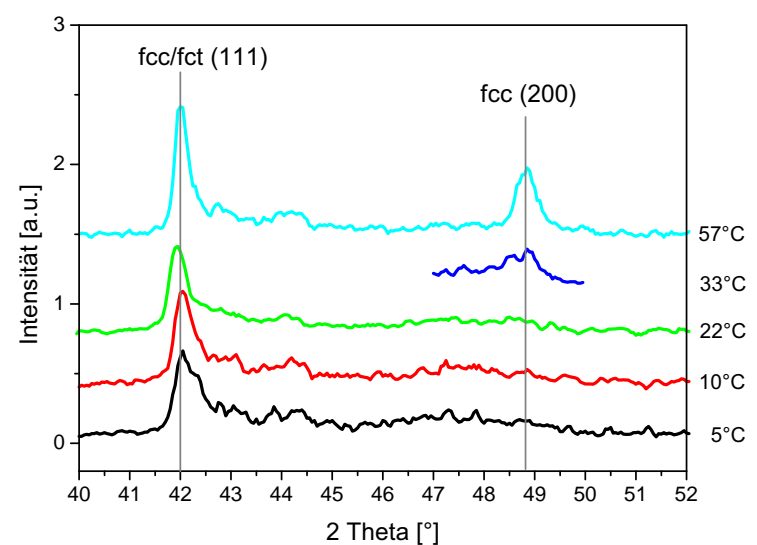

(a) $\mathrm{Fe}_{70,0} \mathrm{Pd}_{23,8} \mathrm{Cu}_{6,2}$ abgeschreckt in Wasser

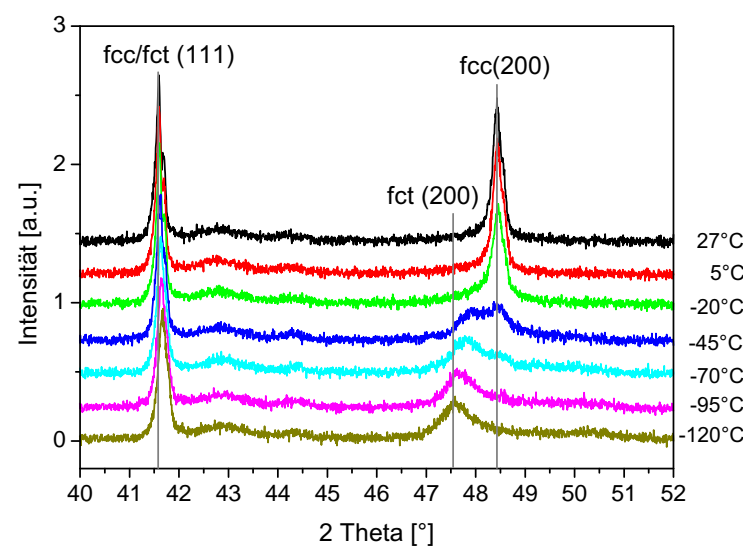

(c) $\mathrm{Fe}_{68,4} \mathrm{Pd}_{29,3} \mathrm{Cu}_{2,3}$ abgekühlt an Luft

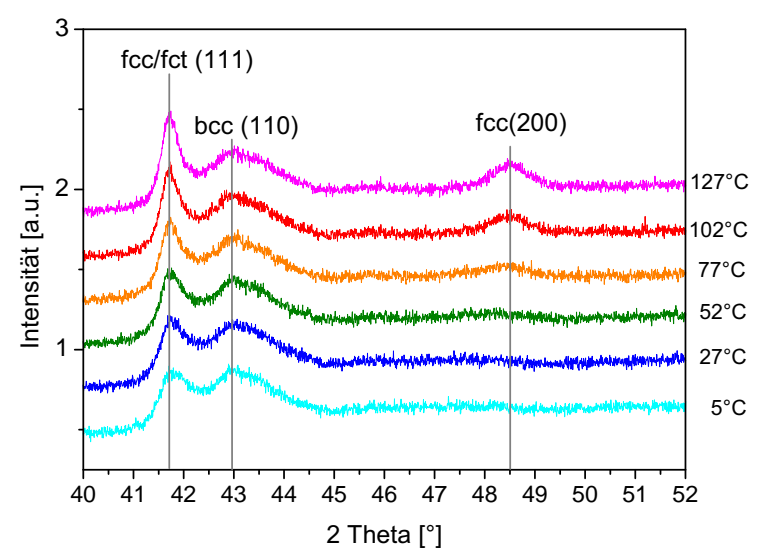

(b) $\mathrm{Fe}_{71,2} \mathrm{Pd}_{27,8} \mathrm{Cu}_{1,0}$ abgeschreckt in Wasser

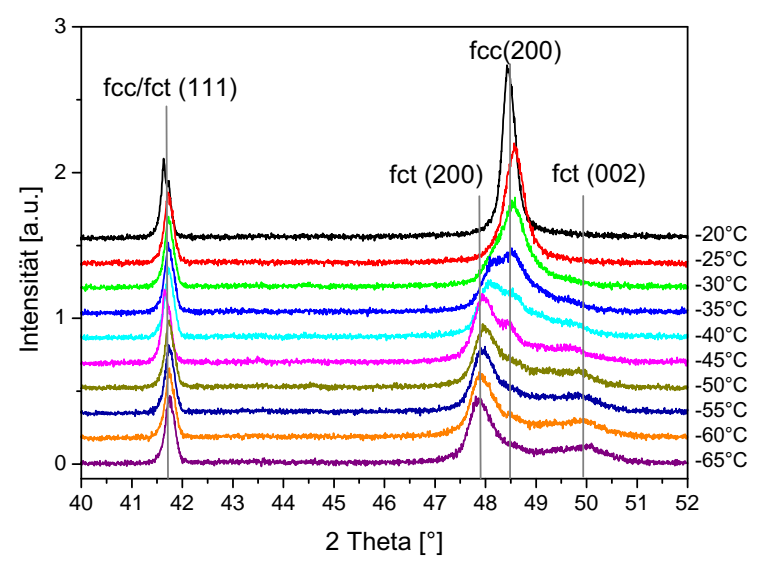

(d) $\mathrm{Fe}_{68,4} \mathrm{Pd}_{29,3} \mathrm{Cu}_{2,3}$ abgeschreckt in Wasser

Abbildung 4.20: Bei der martensitischen Umwandlung kupferhaltiger Proben bleibt die Position des fcc/fct(111)-Peaks etwa konstant, während sich der (200)-Peak verschiebt bzw. aufspaltet. (XRD(T): S.Hamann)

Das Transformationsverhalten kupferhaltiger Proben ähnelt dem der binären Legierung. Die Röntgendiffraktogramme in Abbildung 4.20 zeigen, dass sich auch hier die Position des fcc/fct(111)-Peaks kaum ändert, d.h. die Länge der Raumdiagonalen (und damit auch in erster Näherung das Volumen der Einheitszelle) bleibt im Wesentlichen konstant. In der für eine Stunde bei $850{ }^{\circ} \mathrm{C}$ getemperten und in Wasser abgeschreckten Probe der Zusammensetzung $\mathrm{Fe}_{70,0} \mathrm{Pd}_{23,8} \mathrm{Cu}_{6,2}$ und der an Luft abgekühlten Probe der Zusammensetzung $\mathrm{Fe}_{68,4} \mathrm{Pd}_{29,3} \mathrm{Cu}_{2,3}$ verschiebt sich der (200)-Peak bei der Transformation zu kleineren Winkeln und es bleiben keine nennenswerten Austenitreste bestehen. In der Probe der Zusammensetzung $\mathrm{Fe}_{71,2} \mathrm{Pd}_{27,8} \mathrm{Cu}_{1,0}$ verschwindet der fct(200)-Peak. Der fcc/fct(111)-Peak verändert sich nicht. Auch hier bleibt kein Austenit zurück. Die Übergangstemperatur liegt bei etwa $33^{\circ} \mathrm{C}$ für $\mathrm{Fe}_{70,0} \mathrm{Pd}_{23,8} \mathrm{Cu}_{6,2}$, zwischen $-20^{\circ} \mathrm{C}$ und 


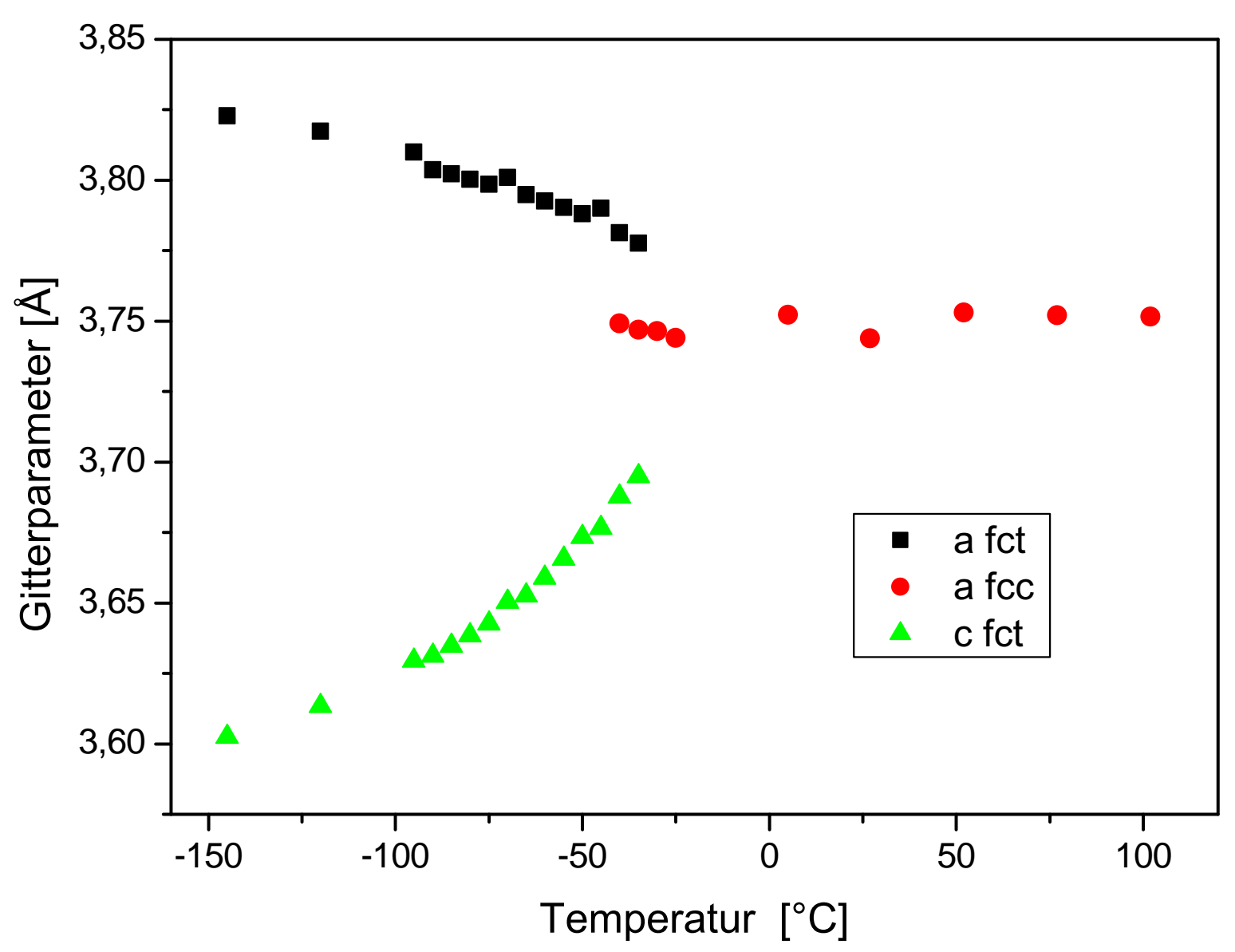

\begin{abstract}
Abbildung 4.21: Bei der in Wasser abgeschreckten Probe der Zusammensetzung $\mathrm{Fe}_{68,4} \mathrm{Pd}_{29,3} \mathrm{Cu}_{2,3}$ geht die kubische Einheitszelle mit drei gleich langen Achsen $a_{f c c}$ bei tiefen Temperaturen in eine tetragonal verzerrte Einheitszelle mit zwei langen Achsen $a_{f c t}$ und einer kurzen Achse $c_{f c t}$ mit minimalem Achsenverhältnis $c / a_{f c t}=0,942$ über.
\end{abstract}

$-45^{\circ} \mathrm{C}$ für die an Luft abgekühlten Probe der Zusammensetzung $\mathrm{Fe}_{68,4} \mathrm{Pd}_{29,3} \mathrm{Cu}_{2,3}$ und bei $77^{\circ} \mathrm{C}$ für $\mathrm{Fe}_{71,2} \mathrm{Pd}_{27,8} \mathrm{Cu}_{1,0}$. Die in Wasser abgeschreckte Probe der Zusammensetzung $\mathrm{Fe}_{68,4} \mathrm{Pd}_{29,3} \mathrm{Cu}_{2,3}$ verhält sich wiederum anders. Im Gegensatz zu den anderen Proben zeigt der (200)-Peak nicht nur eine Verschiebung, sondern tatsächlich eine deutliche Aufspaltung in einen fct(200)/(020)- und einen fct(002)-Peak. Die Übergangstemperatur ist jedoch ähnlich wie bei der an Luft abgekühlten Probe.

Anhand dieser Aufspaltung, die in Abbildung 4.21 gezeigt ist, kann der Übergang der kubischen Einheitszelle mit drei gleich langen Achsen $a_{f c c}=3,75 \pm 0,01 \AA$ zu einer tetragonal verzerrten Einheitszelle mit zwei langen Achsen $a_{f c t}=3,82 \pm 0,01 \AA$ und einer kurzen Achse $c_{f c t}=3,60 \pm 0,01 \AA$ direkt nachvollzogen werden. Die Aufspaltung beginnt bei $M_{s}=-35^{\circ} \mathrm{C}$ und setzt sich bis zum Ende des Messbereichs bei $-145^{\circ} \mathrm{C}$ fort, wo ein Achsenverhältnis von $c / a_{f c t}=0,942 \pm 0,005$ erreicht wird.

\title{
4.3.2 Widerstand
}

Im Zuge des martensitischen Phasenübergangs ändert sich auch der elektrische Widerstand der Proben. Allgemein ist bei metallischen Proben ein Absinken des Widerstandes 


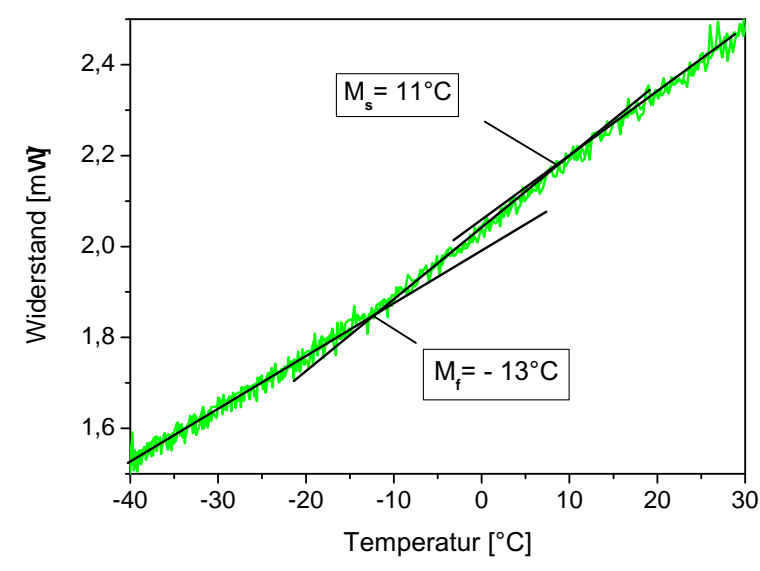

(a) unbehandelt

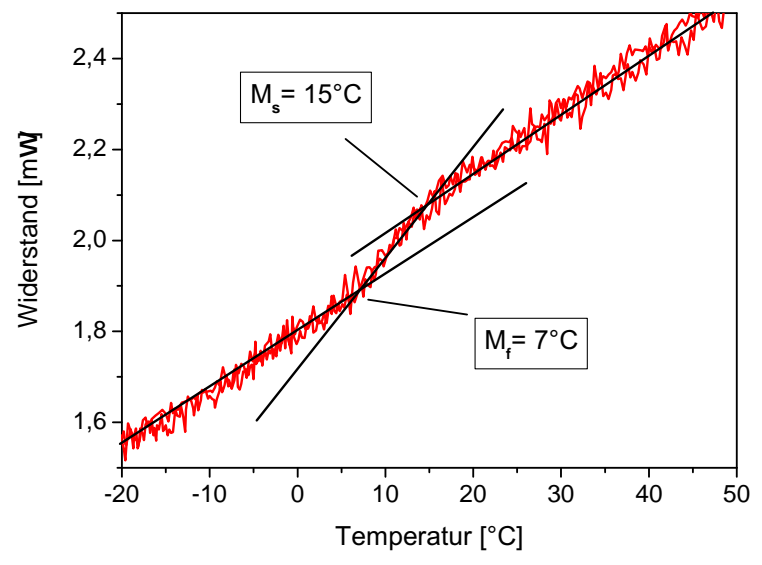

(b) $1000^{\circ} \mathrm{C}$

\begin{abstract}
Abbildung 4.22: Anhand der unbehandelten und der bei $1000{ }^{\circ} \mathrm{C}$ geglühten Probe ist exemplarisch gezeigt, wie die Umwandlungstemperaturen durch Anlegen von Tangenten aus der Widerstandsänderung während der Umwandlung ermittelt werden. (R(T): S.Hamann)
\end{abstract}

mit fallender Temperatur zu erwarten, wie dies auch in Abbildung $4.22 \mathrm{zu}$ beobachten ist. Während der martensitischen Phasenumwandlung fällt der Widerstand etwas steiler ab als sonst. Da dieser Effekt nicht sehr stark ausgeprägt ist, wurden die Umwandlungstemperaturen ermittelt, indem Geraden an die Widerstandskurven angelegt wurden, und zwar jeweils eine in den Bereichen konstanten Abfalls und eine weitere in jenem Bereich, in dem der Widerstand verstärkt abfällt. Aus den Schnittpunkten der Geraden können die Umwandlungstemperaturen abgelesen werden. In Abbildung 4.22 ist dies exemplarisch an den Widerstandskurven einer unbehandelten und einer für 15 Minuten bei $1000{ }^{\circ} \mathrm{C}$ geglühten Probe der Zusammensetzung $\mathrm{Fe}_{70} \mathrm{Pd}_{30}$ gezeigt.

In Abbildung 4.23 sind die Widerstandskurven der bei verschiedenen Temperaturen geglühten $\mathrm{Fe}_{70} \mathrm{Pd}_{30}$-Proben zusammengefasst. Wie schon bei der strukturellen Charakterisierung fällt auf, dass die bei $700{ }^{\circ} \mathrm{C}$ getemperte Probe keinen Übergang zeigt. Bei allen anderen Proben ist ein (zum Teil schwacher) Übergang zu erkennen, der mit steigender Glühtemperatur deutlicher wird. Die beim Heizen und Kühlen aufgenommenen Widerstandskurven liegen jeweils aufeinander, es ist also keine Hysterese zu beobachten und Martensitstart- und Austenitendtemperatur sind identisch. Die in Tabelle 4.1 zusammengefassten Übergangstemperaturen, insbesondere die Martensitendtmperatur $M_{f}$, weisen mit steigender Glühtemperatur einen leichten Anstieg auf.

\begin{tabular}{c|c|c|c|c|r|r} 
Glühtemperatur & \multicolumn{1}{|c|}{ unbeh. } & \multicolumn{1}{|c|}{$600{ }^{\circ} \mathrm{C}$} & $700{ }^{\circ} \mathrm{C}$ & $800^{\circ} \mathrm{C}$ & \multicolumn{1}{|c}{$900{ }^{\circ} \mathrm{C}$} & \multicolumn{1}{|c}{$1000^{\circ} \mathrm{C}$} \\
\hline$M_{s} / A_{f}$ & $11 \pm 1{ }^{\circ} \mathrm{C}$ & $9 \pm 1{ }^{\circ} \mathrm{C}$ & - & $9 \pm 1{ }^{\circ} \mathrm{C}$ & $16 \pm 1{ }^{\circ} \mathrm{C}$ & $15 \pm 1{ }^{\circ} \mathrm{C}$ \\
$M_{f} / A_{s}$ & $-13 \pm 1{ }^{\circ} \mathrm{C}$ & $-5 \pm 1{ }^{\circ} \mathrm{C}$ & - & $-3 \pm 1{ }^{\circ} \mathrm{C}$ & $4 \pm 1{ }^{\circ} \mathrm{C}$ & $7 \pm 1{ }^{\circ} \mathrm{C}$
\end{tabular}

Tabelle 4.1: Übergangstemperaturen $M_{s} / A_{f}$ und $M_{f} / A_{s}$, wie sie aus den Widerstandsmessungen gewonnen wurden. 


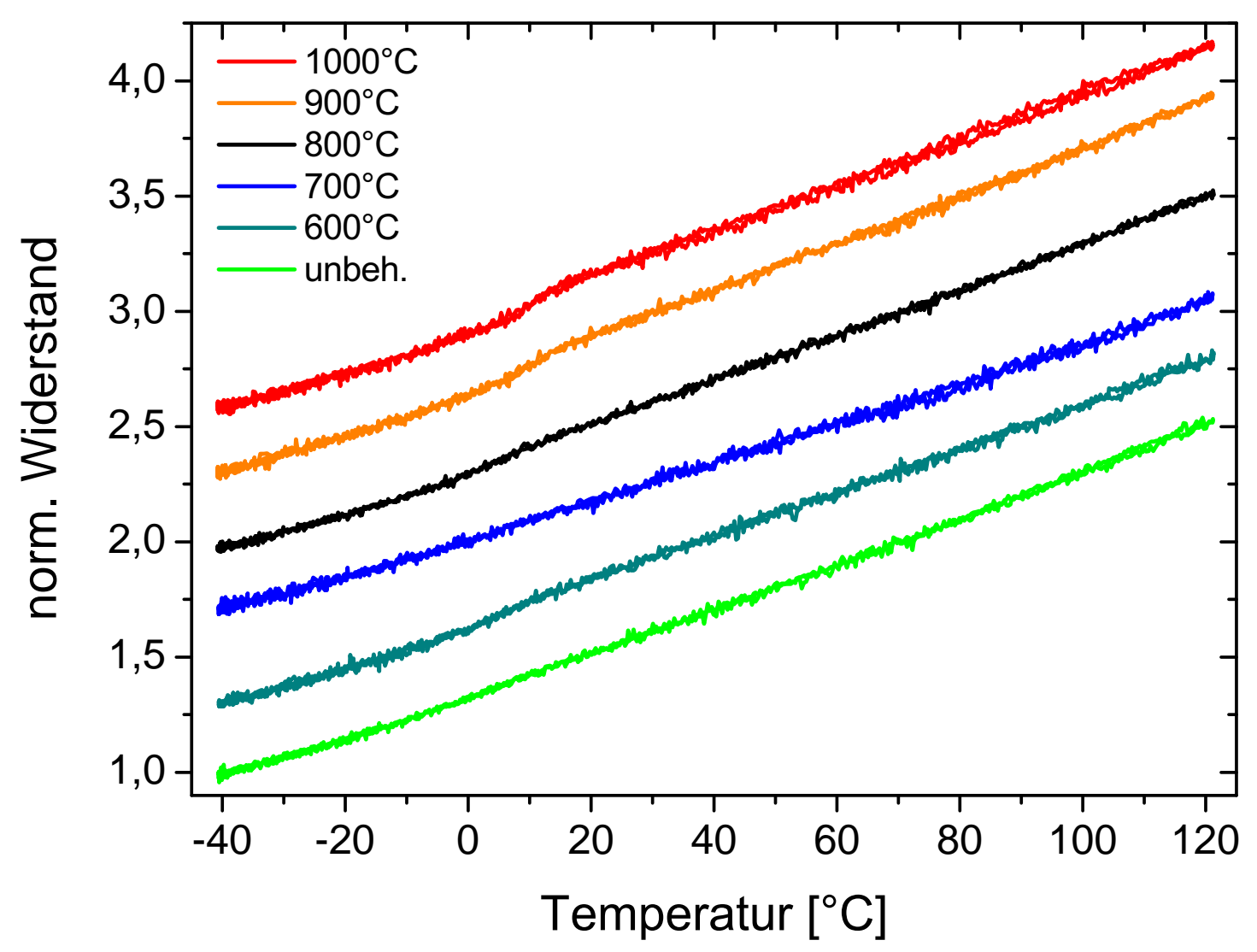

Abbildung 4.23: Alle Proben zeigen einen verstärkten Abfall des Widerstandes während des Phasenübergangs, der mit steigender Glühtemperatur deutlicher wird. $(\mathrm{R}(\mathrm{T})$ : S.Hamann)

\subsubsection{Magnetisierung}

Die magnetischen Eigenschaften metallischer Proben sind sehr eng mit deren atomarer Konfiguration verknüpft. Eine Änderung der Kristallstruktur kann somit eine Änderung ihrer Magnetisierung nach sich ziehen. Daher wurde die Änderung der Magnetisierung während des martensitischen Phasenübergangs in einem Magnetfeld von $30 \mathrm{mT}$ sowohl in Abhängigkeit von der Zusammensetzung als auch von der Glühtemperatur untersucht.

In Abbildung 4.24 sind die Magnetisierungskurven von Proben mit verschiedenem Palladiumgehalt zwischen $28 \%$ und $32 \%$ zu sehen. Für Proben der Zusammensetzung $\mathrm{Fe}_{70} \mathrm{Pd}_{30}$ wurde zusätzlich der Einfluss einer Glühbehandlung bei verschiedenen Temperaturen untersucht. Die Magnetisierungskurven dieser Proben sind in Abbildung 4.25 zu sehen. Alle transformierenden Proben zeigen ein grundsätzlich ähnliches Verhalten. Bei Temperaturen oberhalb der Übergangstemperatur ist die Magnetisierung näherungsweise konstant. Ab einer gewissen Temperatur, die mit dem Einsetzen der Peakverschiebung in den Röntgendiffraktogrammen zusammenfällt und daher als Martensitstarttemperatur identifiziert wird, beginnt die Magnetisierung zu fallen und sinkt bis zum Ende des Messbereichs kontinuierlich weiter ab. Beim Aufwärmen steigt sie in gleichem Maße wieder an. Bei den meisten Proben konnte eine Hysterese beobachtet 


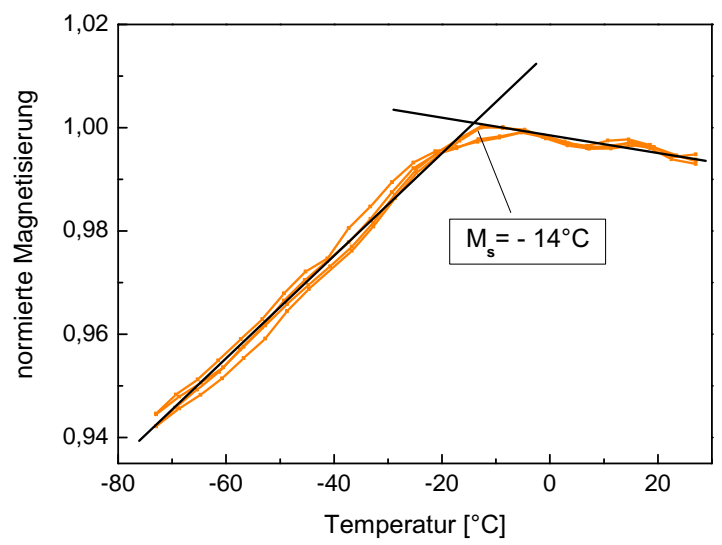

(a) $28 \% \mathrm{Pd}$

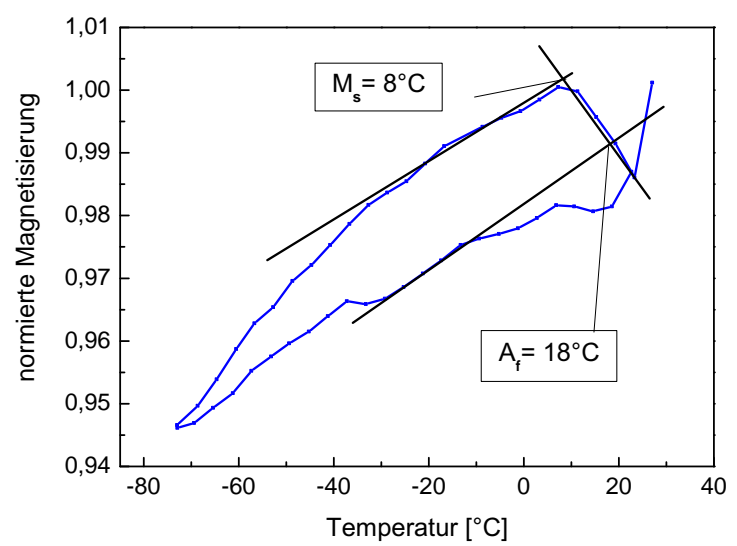

(c) $31 \% \mathrm{Pd}$

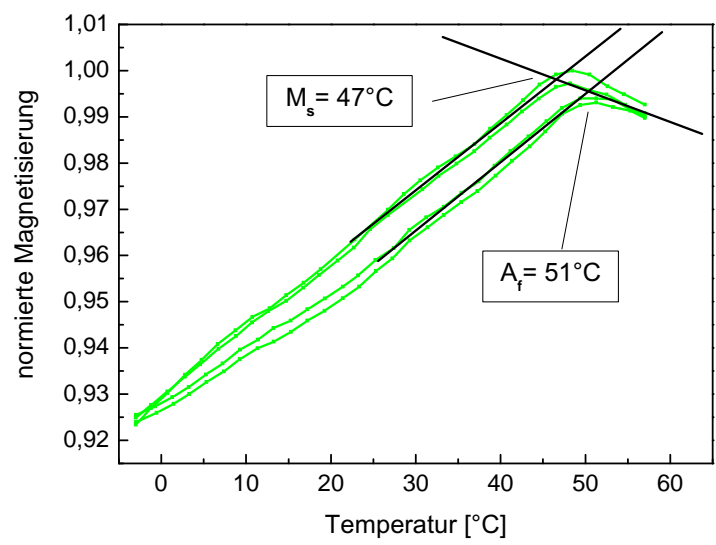

(b) $29 \% \mathrm{Pd}$

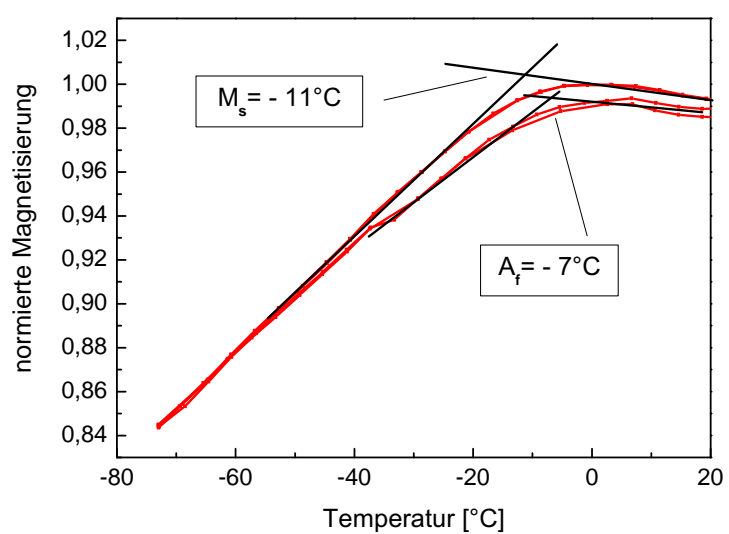

(d) $32 \% \mathrm{Pd}$

Abbildung 4.24: Mit Beginn der Phasenumwandlung bei $M_{s}$ beginnt die Magnetisierung zu fallen. Je höher der Palladiumgehalt, desto höher liegt die Umwandlungstemperatur.

werden, so dass eine Bestimmung von $A_{f}$ unabhängig von $M_{s}$ möglich war. Das Ende der martensitischen Transformation kann aus den Magnetisierungskurven jedoch nicht ohne Weiteres bestimmt werden, da diese kein charakteristisches Merkmal aufweisen, das dem Ende des Übergangs zugeordnet werden kann.

Die Probe mit einem Palladiumgehalt von $28 \%$ ist hier gesondert zu betrachten. Da sie bei Zimmertemperatur in der bcc-Phase vorliegt, kann der Abfall der Magnetisierung nicht mit dem martensitischen Phasenübergang in Verbindung gebracht werden. Hier muss ein anderer Mechanismus zugrunde liegen. Die Proben mit einem Palladiumgehalt von $29 \%$ oder mehr in Abbildung 4.24 zeigen alle einen mehr oder weniger deutlich ausgeprägten Übergang. Die Übergangstemperatur sinkt mit steigendem Palladiumgehalt von $M_{s}=47 \pm 2{ }^{\circ} \mathrm{C}$ auf $M_{s}=-11 \pm 2{ }^{\circ} \mathrm{C}$ ab.

Die Magnetisierungskurve einer unbehandelten Probe mit einem Palladiumgehalt von $30 \%$ ist in Abbildung 4.25 gemeinsam mit den Kurven geglühter Proben gezeigt. Zunächst fällt wieder auf, dass die bei $700{ }^{\circ} \mathrm{C}$ geglühte Probe keinen Phasenübergang, sondern lediglich einen leichten Abfall der Magnetisierung zeigt. Die anderen Proben zeigen einen Übergang mit Hysterese, der mit steigender Glühtemperatur deutlicher 


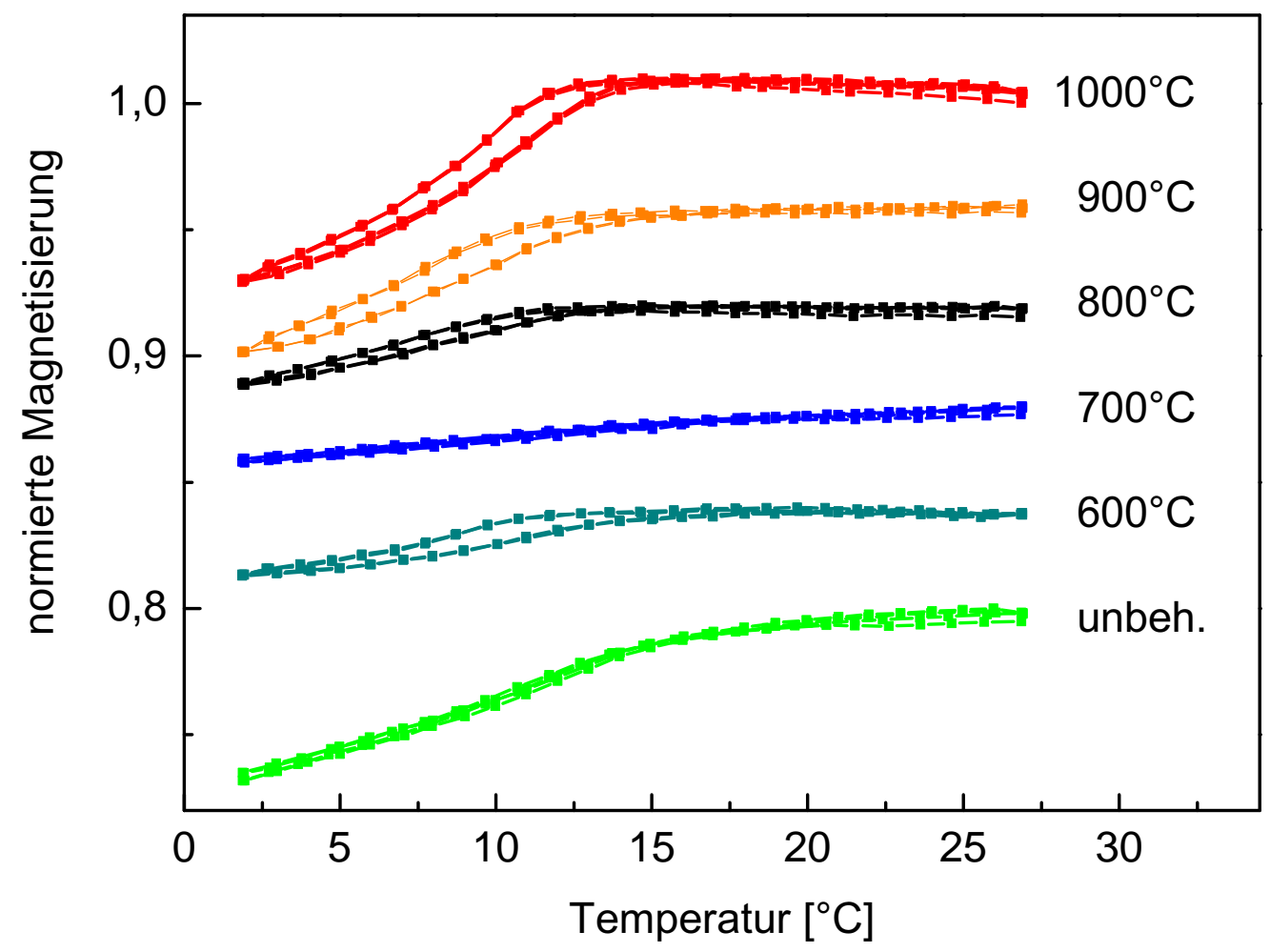

Abbildung 4.25: Bei verschiedenen Temperaturen geglühte Proben der Zusammensetzung $\mathrm{Fe}_{70} \mathrm{Pd}_{30}$ : Alle transformierenden Proben zeigen einen deutlichen Abfall der Magnetisierung mit Beginn des martensitischen Phasenübergangs.

wird und sich zu höheren Temperaturen verschiebt. Einzige Ausnahme (neben der bei $700{ }^{\circ} \mathrm{C}$ geglühten Probe) bildet die unbehandelte Probe, die kaum eine Hysterese aufweist und bei höheren Temperaturen transformiert als die anderen Proben. Die Martensitstart- und Austenitendtemperaturen $M_{s}$ und $A_{f}$, die aus den Magnetisierungskurven gewonnen wurden, sind in Tabelle 4.2 zusammengefasst.

\begin{tabular}{c|c|c|c|c|c|c} 
Glühtemperatur & unbeh. & $600{ }^{\circ} \mathrm{C}$ & $700{ }^{\circ} \mathrm{C}$ & $800{ }^{\circ} \mathrm{C}$ & $900{ }^{\circ} \mathrm{C}$ & $1000{ }^{\circ} \mathrm{C}$ \\
\hline$M_{s}$ & $16 \pm 2{ }^{\circ} \mathrm{C}$ & $11 \pm 2{ }^{\circ} \mathrm{C}$ & - & $11 \pm 2{ }^{\circ} \mathrm{C}$ & $12 \pm 2{ }^{\circ} \mathrm{C}$ & $12 \pm 2{ }^{\circ} \mathrm{C}$ \\
$A_{f}$ & $16 \pm 2{ }^{\circ} \mathrm{C}$ & $15 \pm 2{ }^{\circ} \mathrm{C}$ & - & $13 \pm 2{ }^{\circ} \mathrm{C}$ & $14 \pm 2{ }^{\circ} \mathrm{C}$ & $14 \pm 2{ }^{\circ} \mathrm{C}$
\end{tabular}

Tabelle 4.2: Übergangstemperaturen $M_{s}$ und $A_{f}$, wie sie aus den Magnetisierungskurven der geglühten $\mathrm{Fe}_{70} \mathrm{Pd}_{30}$-Proben gewonnen wurden.

Die Zugabe von Kupfer beeinflusst das temperaturabhängige Verhalten der Magnetisierung nicht signifikant. Abbildung 4.26 zeigt Magnetisierungskurven von Proben der Zusammensetzung $\mathrm{Fe}_{68,4} \mathrm{Pd}_{29,3} \mathrm{Cu}_{2,3}$ und $\mathrm{Fe}_{70,0} \mathrm{Pd}_{23,8} \mathrm{Cu}_{6,2}$, die für eine Stunde bei $850^{\circ} \mathrm{C}$ geglüht und anschließend in Wasser abgeschreckt wurden. Die Übergangstemperatur der Probe mit der Zusammensetzung $\mathrm{Fe}_{68,4} \mathrm{Pd}_{29,3} \mathrm{Cu}_{2,3}$ liegt deutlich tiefer als diejenigen der binären Proben, bei einer Zusammensetzung von $\mathrm{Fe}_{70,0} \mathrm{Pd}_{23,8} \mathrm{Cu}_{6,2}$ ist sie hingegen eher erhöht. Der gesamte Abfall der Magnetisierung ist ähnlich ausgeprägt wie in der binären Legierung. 


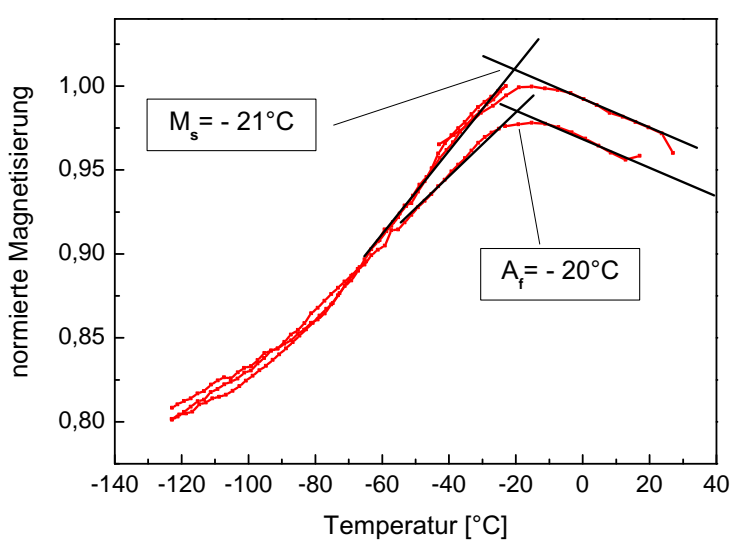

(a) $\mathrm{Fe}_{68,4} \mathrm{Pd}_{29,3} \mathrm{Cu}_{2,3}$

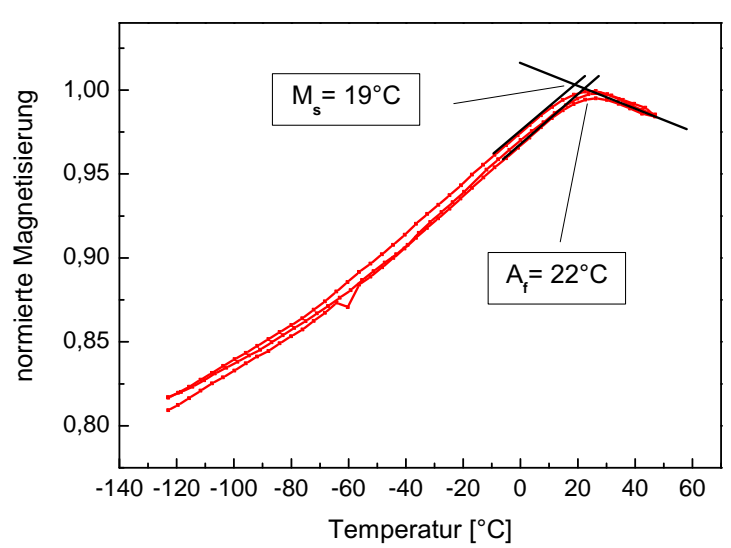

(b) $\mathrm{Fe}_{70,0} \mathrm{Pd}_{23,8} \mathrm{Cu}_{6,2}$

Abbildung 4.26: Die Zugabe von Kupfer verändert das temperaturabhängige Verhalten der Magnetisierung nicht signifikant gegenüber binären Proben.

\subsubsection{Mechanische Eigenschaften}

Mit Hilfe des in Kapitel 3 beschriebenen Vibrating-Reed-Experiments und mit Zugversuchen wurde ein Einblick in die mechanischen Eigenschaften binärer Eisen-PalladiumSplats gewonnen. Dabei zeigte sich, dass sich das mechanische Verhalten für große und kleine Dehnungen deutlich unterscheidet. Daher soll zunächst das rein elastische Verhalten bei sehr kleinen Dehnungen in einem Vibrating-Reed-Experiment vorgestellt werden. Anschließend wird das elastische und plastische Verhalten bei deutlich größeren Dehnungen gezeigt, wie sie in Zugversuchen erreicht werden.

In dem Vibrating-Reed-Experiment kann aus der Eigenfrequenz $f$ der freien Schwingung mit Gleichung 3.19 der Elastizitätsmodul E mit

$$
E=12\left(\frac{2 \pi}{1,875^{2}}\right) \cdot \frac{L^{4}}{d^{2}} \rho f^{2}
$$

für eine Probe der Länge $L$, Dicke $d$ und Dichte $\rho=8,9 \cdot 10^{3} \mathrm{~kg} / \mathrm{m}^{3(1)}$ gewonnen werden. Im Prinzip müsste hier der Einfluss der Dämpfung auf die Eigenfrequenz $f_{0}$ der ungedämpften Schwingung durch $f=\sqrt{f_{0}^{2}-(\lambda / 2 \pi)^{2}}$ berücksichtigt werden. Im Rahmen der Messgenauigkeit ist jedoch auch bei maximaler Dämpfung kein Unterschied zwischen $f$ und $f_{0}$ zu erkennen. Für eine austenitische $\mathrm{Fe}_{70} \mathrm{Pd}_{30}$-Probe der Länge $L=16,7 \pm 0,5 \mathrm{~mm}$ und Dicke $d=47 \pm 8 \mu \mathrm{m}$ ergibt sich mit einer Frequenz von $f=76,7 \pm 0,1 \mathrm{~Hz}$ bei Zimmertemperatur ein Elastizitätsmodul von $E=72 \pm 10 \mathrm{GPa}$, für eine martensitische $\mathrm{Fe}_{71} \mathrm{Pd}_{29}$-Probe $(L=16,8 \pm 0,5 \mathrm{~mm}, d=59 \pm 6,5 \mathrm{~mm}, f=$ $112,8 \pm 0,2 \mathrm{~Hz})$ liegt er bei $E=100 \pm 11 \mathrm{GPa}$.

Der Elastizitätsmodul ändert sich während des martensitischen Phasenüberganges stark. Daher bietet es sich an, die relative Änderung des Elastizitätsmoduls $\Delta E / E_{0}$ zu

\footnotetext{
${ }^{(1)}$ Die Dichte wurde abgeschätzt aus den molaren Massen von Eisen und Palladium und dem Volumen der Einheitszelle des Austenits.
} 


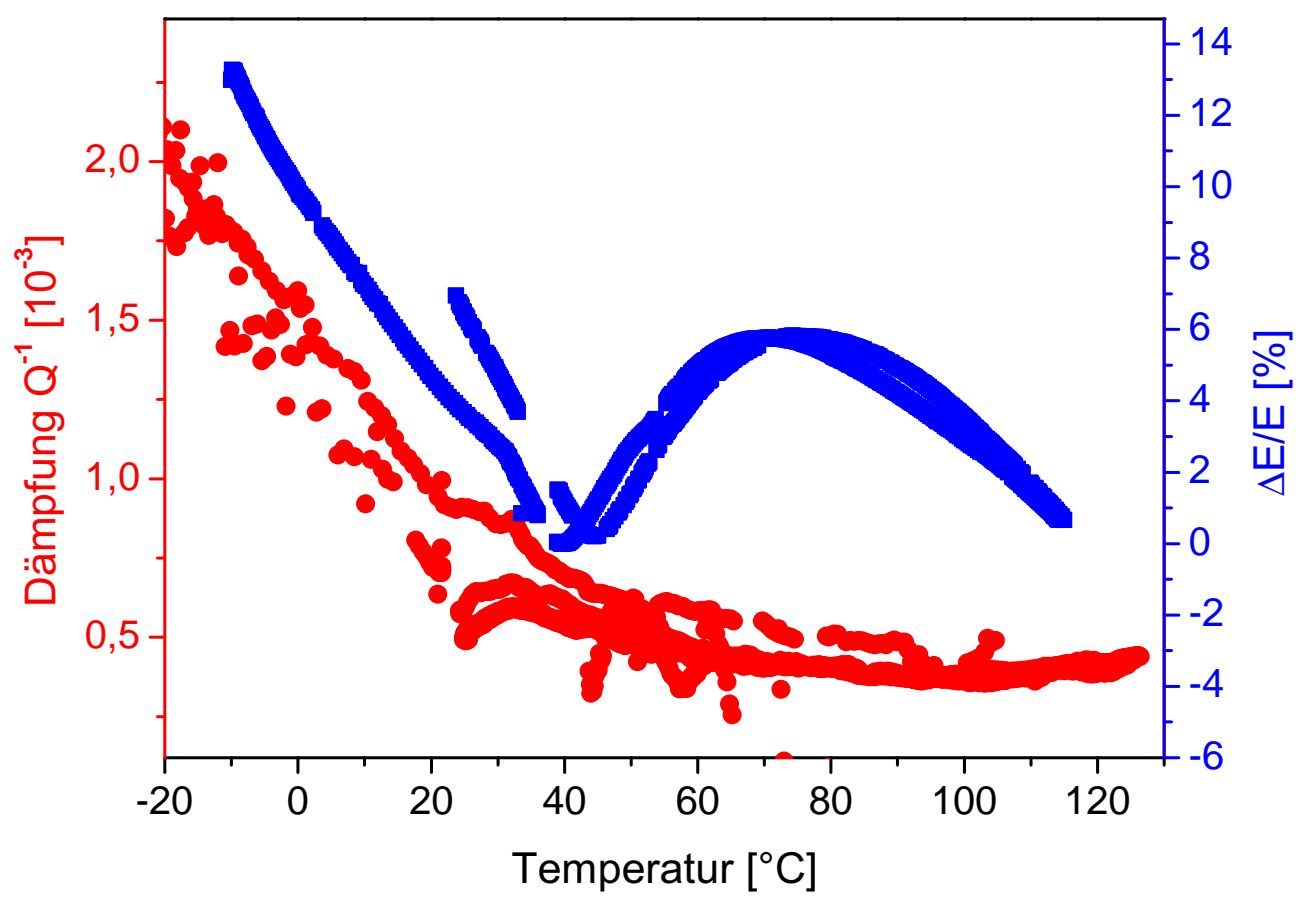

Abbildung 4.27: Proben der Zusammensetzung $\mathrm{Fe}_{71} \mathrm{Pd}_{29}$ zeigen vor und nach dem Phasenübergang einen Anstieg des Elastizitätsmoduls. Während des Übergangs zwischen $70^{\circ} \mathrm{C}$ und $40^{\circ} \mathrm{C}$ fällt er jedoch stark ab. Die Dämpfung steigt nach der Phasenumwandlung steil an.

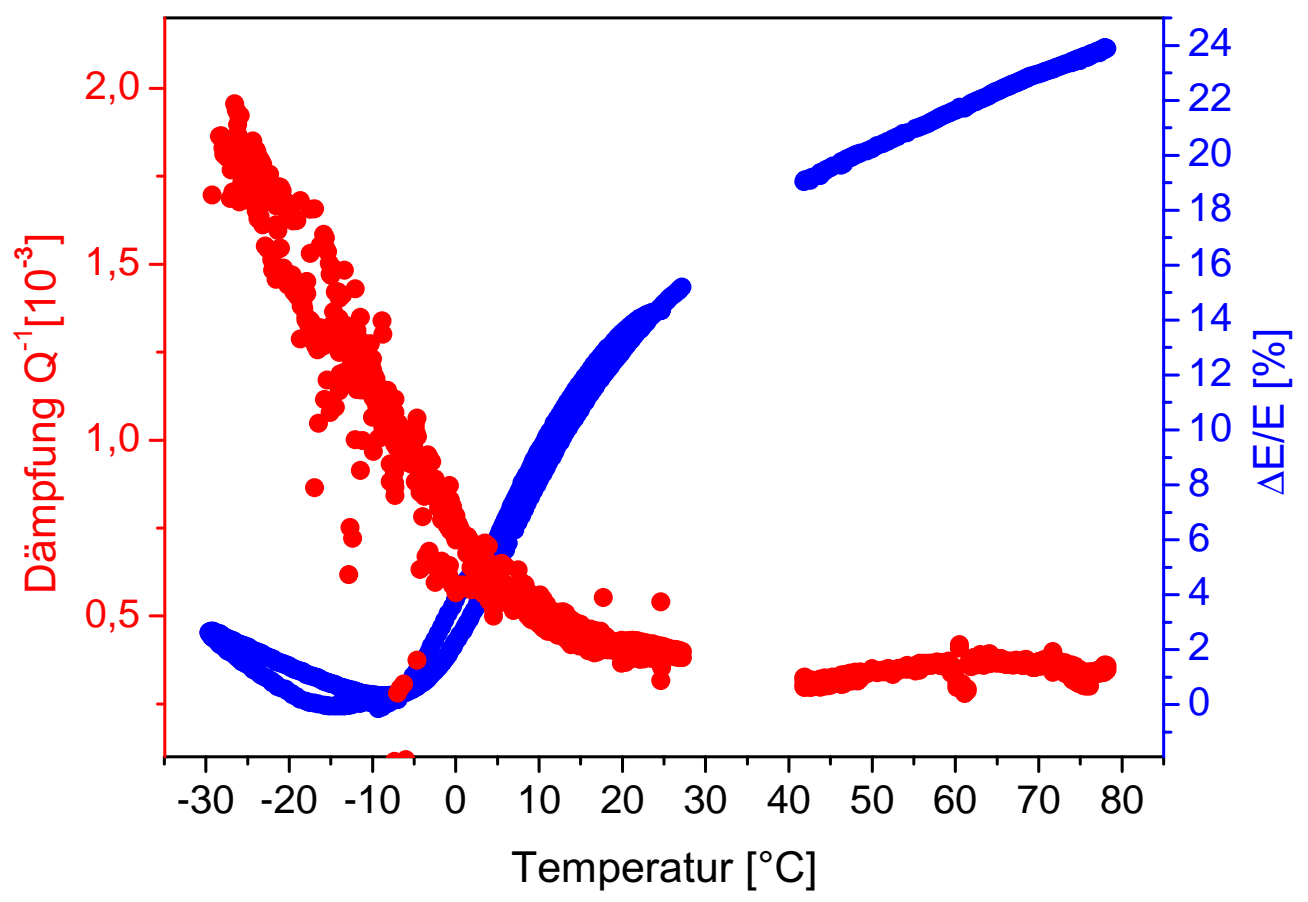

Abbildung 4.28: In Proben der Zusammensetzung $\mathrm{Fe}_{70} \mathrm{Pd}_{30}$ fällt der Elastizitätsmodul vor und während des Phasenübergangs stark ab. Dieser endet etwa bei $-10^{\circ} \mathrm{C}$. Die Dämpfung steigt nach der Phasenumwandlung steil an. 
betrachten. Zur besseren Vergleichbarkeit von Proben unterschiedlicher Zusammensetzung, die entsprechend bei verschiedenen Temperaturen transformieren, bezieht sich die Änderung jeweils auf den kleinsten Wert des Elastizitätsmoduls, der am Ende des Phasenübergangs auftritt.

In Abbildung 4.27 ist die temperaturabhängige Änderung der Dämpfung $Q^{-1}$ und des Elastizitätsmoduls $\Delta E / E_{0}$ einer Probe der Zusammensetzung $\mathrm{Fe}_{71} \mathrm{Pd}_{29}$ dargestellt. Bei Temperaturen deutlich oberhalb und deutlich unterhalb des Phasenübergangs steigt der Elastizitätsmodul mit sinkender Temperatur etwa linear an. Während des Phasenübergangs fällt er dagegen um etwa $6 \%$ ab und erreicht ein Minimum bei ca. $40^{\circ} \mathrm{C}$. Die Dämpfung der Probe hingegen liegt bei Temperaturen oberhalb des Phasenüberganges weitestgehend konstant bei $0,5 \cdot 10^{-3}$. Während des Phasenüberganges beginnt sie leicht anzusteigen und nimmt zu tiefen Temperaturen hin stetig weiter zu.

Der in Abbildung 4.28 dargestellte Elastizitätsmodul einer Probe der Zusammensetzung $\mathrm{Fe}_{70} \mathrm{Pd}_{30}$ verhält sich anders. Bereits bei Temperaturen weit oberhalb der Martensitstarttemperatur beginnt er deutlich abzufallen. Während des Phasenüberganges ist der Abfall am stärksten ausgeprägt. Über den gesamten Messbereich beträgt die Änderung $24 \%$. Bei etwa $-10^{\circ} \mathrm{C}$ wird ein Minimum erreicht. Danach steigt der Elastizitätsmodul wieder leicht an. Die Dämpfung ist bei hohen Temperaturen annähernd konstant und steigt nach dem Phasenübergang steil an.

Ergänzend zu den Vibrating-Reed-Messungen wurden Spannungs-Dehnungskurven an einer Probe der Zusammensetzung $\mathrm{Fe}_{70} \mathrm{Pd}_{30}$ aufgenommen, die 150 Stunden bei $900{ }^{\circ} \mathrm{C}$ geglüht und anschließend in Wasser abgeschreckt wurde. Zunächst wurde bei verschiedenen Temperaturen oberhalb, unterhalb und während des Phasenübergangs das Verhalten für kleine Dehnungen bis etwa 1\% untersucht. Diese Messungen sind in Abbildung $4.29 \mathrm{zu}$ sehen. Anschließend wurden die Messungen bei größeren Dehnungen bis zum Materialversagen wiederholt (Abbildung 4.30 und 4.31). Um den Anteil der elastischen Verformung des Gestänges der Zugprüfanlage zu identifizieren, wurde dabei die Längenänderung zusätzlich mit dem Videoextensometer überprüft. Dabei ergab sich, dass entgegen der Herstellerangaben ein signifikanter Anteil der von der Dehnungsmesszelle aufgenommenen Dehnung auf elastische Verformung des Gestänges zurückzuführen war. Dadurch erscheint der Anstieg der Spannungs-Dehnungskurve zu flach. Um diesen Fehler zu eliminieren, wurde für die Messungen bei großen Dehnungen in den Abbildungen 4.30 und 4.31 die Spannung gegen die mit dem Videoextensometer ermittelte Dehnung aufgetragen. Da die Messungen bei kleinen Dehnungen nicht mit dem Videoextensometer überprüft worden waren, wurden hier die mit der Dehnungsmesszelle ermittelten Dehnungen so reskaliert, dass der aus dem Abfall der Spannung beim Entlasten gewonnene Elastizitätsmodul bei beiden Messungen übereinstimmt. Der Nullpunkt der Dehnung bleibt dabei festgelegt als derjenige Punkt, an dem die Spannung anzusteigen beginnt. Wegen der Unsicherheit von etwa 20\%, mit der die Dehnungen nach der Reskalierung behaftet sind, wurde aus diesen Messungen kein Elastizitätsmodul bestimmt.

In Abbildung 4.29 ist der Verlauf der Spannung für kleine Dehnungen bis etwa $1 \%$ bei verschiedenen Temperaturen zu sehen. Es ist klar zu erkennen, dass sich die Kurven, die bei Temperaturen deutlich oberhalb, deutlich unterhalb und während des Phasenübergangs aufgenommen wurden, qualitativ unterscheiden. Bei $40{ }^{\circ} \mathrm{C}$, wenn sich die Probe in der austenitischen Phase befindet, steigt die Spannung linear mit der Deh- 


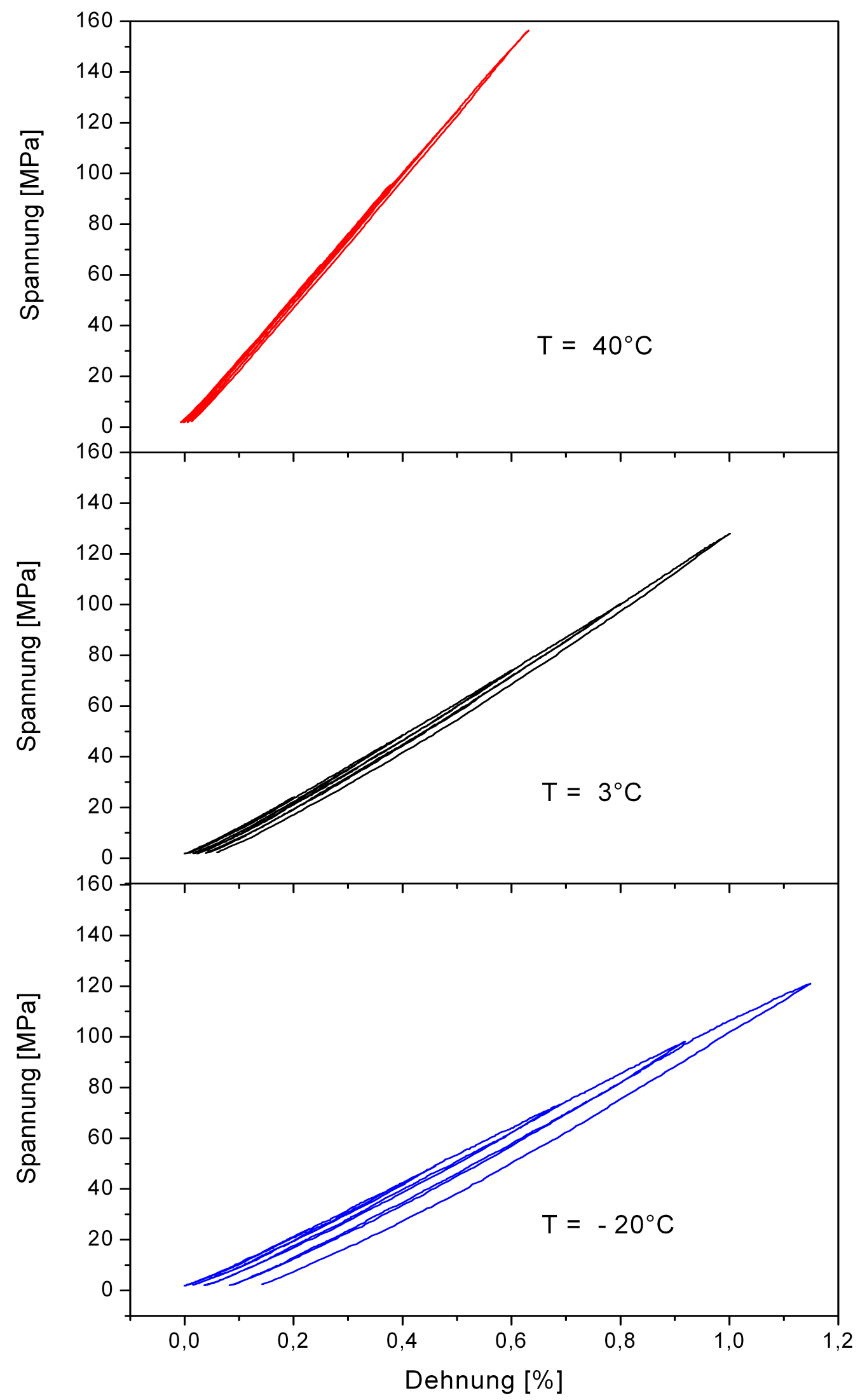

Abbildung 4.29: Im Austenit (bei $40^{\circ} \mathrm{C}$ ) steigt die Spannung linear mit der Dehnung an. Diese geht beim Entlasten vollständig zurück. Wenn die Probe teilweise oder vollständig martensitisch ist $\left(3{ }^{\circ} \mathrm{C}\right.$ und $\left.-20^{\circ} \mathrm{C}\right)$, bleibt nach dem Entlasten ein Teil der Dehnung bestehen. (Messung: C.Bechtold) 
nung an, bis sie bei einer Dehnung von etwa 0,65\% einen Wert von $150 \mathrm{MPa}$ erreicht. Bei dieser geringen Dehnung verhält sich die Probe fast vollständig elastisch und beim Entlasten bleibt keine signifikante Dehnung bestehen. Bei $3{ }^{\circ} \mathrm{C}$, also während des Phasenüberganges, sind die Spannungs-Dehnungskurven immer noch weitestgehend linear, die Steigung ist jedoch etwas flacher. Beim Entlasten bleibt eine geringe Dehnung zurück. Beim erneuten Dehnen folgt die Spannung im Wesentlichen dem Verlauf des vorherigen Entlastens. Bei der maximalen Dehnung des letzten Zyklus flacht die Steigung der Spannungs-Dehnungskurve etwas ab und nach dem Entlasten bleibt eine geringfügig größere Dehnung der Probe bestehen. Da zu dieser Temperatur keine Messungen mit dem Videoextensometer vorliegen, wurde die Reskalierung der Dehnungsachse grob abgeschätzt. Bei $-20^{\circ} \mathrm{C}$, wenn die Probe vollständig martensitisch ist, verhält sie sich qualitativ ähnlich wie bei $3^{\circ} \mathrm{C}$. Das plastische Verhalten ist jedoch deutlicher ausgeprägt und schon bei sehr kleinen Dehnungen unter $1 \%$ bleibt beim Entlasten eine signifikante Dehnung von etwas mehr als 0,1\% bestehen. Der Abfall der Spannung erfolgt dabei etwa mit der gleichen Steigung wie in den vorherigen Zyklen.

Die Abbildungen 4.30 und 4.31 zeigen, wie sich die Spannung bei größeren Dehnungen verhält. Bei $40^{\circ} \mathrm{C}$, wenn die $\mathrm{Fe}_{70} \mathrm{Pd}_{30}$-Probe vollständig austenitisch ist, steigt die Spannung zunächst sehr stark mit der Dehnung an. Beim Entlasten verschwindet die Dehnung fast vollständig. Die Probe verhält sich also im Wesentlichen elastisch. Ab einer Dehnung von etwa $1 \%$ flacht die Kurve deutlich ab und beim Entlasten bleibt eine signifikante plastische Dehnung bestehen. Beim erneuten Dehnen folgt die Spannung zunächst dem Verlauf des vorherigen Entlastens. Sobald der Endzustand des letzten Dehnungszyklus erreicht wird, flacht die Steigung der Spannungs-Dehnungskurve ab. Beim erneuten Entlasten bleibt eine größere plastische Dehnung bestehen. Durch Anpassen einer Geraden an den linearen Teil des Spannungsabfalls beim Entlasten kann ein Elastizitätsmodul von $25 \pm 5 \mathrm{GPa}$ gewonnen werden. Das Material ist also sehr weich und duktil.

Bei $-10{ }^{\circ} \mathrm{C}$ ist die $\mathrm{Fe}_{70} \mathrm{Pd}_{30}$-Probe vollständig martensitisch. Anhand der SpannungsDehnungs-Kurve in Abbildung 4.31 fällt sofort auf, dass sie bei dieser Temperatur wesentlich weicher ist und das plastische Verhalten früher einsetzt. Der Anstieg der Spannung mit der Dehnung ist deutlich flacher. Auch geringe Dehnungen gehen beim Entlasten nicht vollständig zurück. Bei einer Dehnung von etwa $2 \%$ wird der Anstieg der Spannung noch flacher und beim Entlasten geht die Dehnung unterhalb einer Spannung von $25 \mathrm{MPa}$ deutlich stärker zurück. Aufnahmen mit dem Videoextensometer zeigen, dass sich hier die Probe leicht verdrillt. Bei einer Dehnung von $4 \%$ fällt die Spannung plötzlich ab. Bei weiteren Dehnungszyklen verhält sich die Probe wie zuvor, erscheint jedoch weicher und verdrillt sich beim Entlasten stärker. Daher ist davon auszugehen, dass der Spannungsabfall durch ein Einreißen der Probe verursacht wurde, weshalb die Kurve nur bis zu einer Dehnung von $4 \%$ gezeigt wird. Bei einer Dehnung von $8 \%$ versagt das Material vollständig. Aus dem Rückgang der Spannung beim Entlasten vor dem Einreißen der Probe wurde ein Elastizitätsmodul von $13 \pm 3 \mathrm{GPa}$ gewonnen. Im martensitischen Zustand ist das Material also ebenfalls duktil und noch weicher als im Austenit.

An unbehandelten Proben der Zusammensetzung $\mathrm{Fe}_{70} \mathrm{Pd}_{30}$ und $\mathrm{Fe}_{71} \mathrm{Pd}_{29}$ wurde auch die magnetfeldinduzierte Dehnung bei Zimmertemperatur gemessen (Abbildung 4.32). Die Längenänderung der martensitischen $\mathrm{Fe}_{71} \mathrm{Pd}_{29}$-Probe liegt bei der schrittweisen 


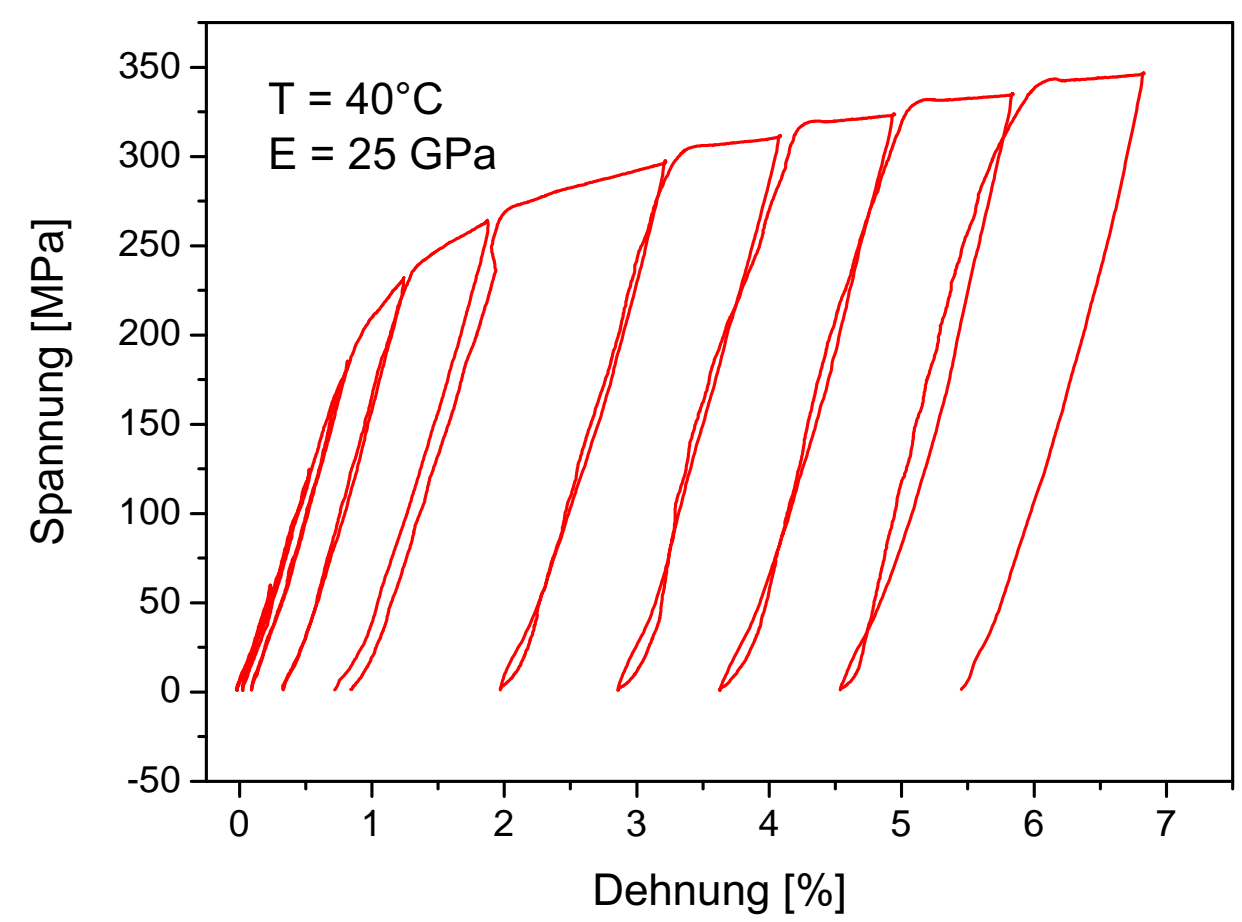

Abbildung 4.30: Bei Dehnungen unter $1 \%$ verhält sich die austenitische Probe elastisch, bei größeren Dehnungen kommt plastisches Verhalten hinzu. (Messung: C.Bechtold)

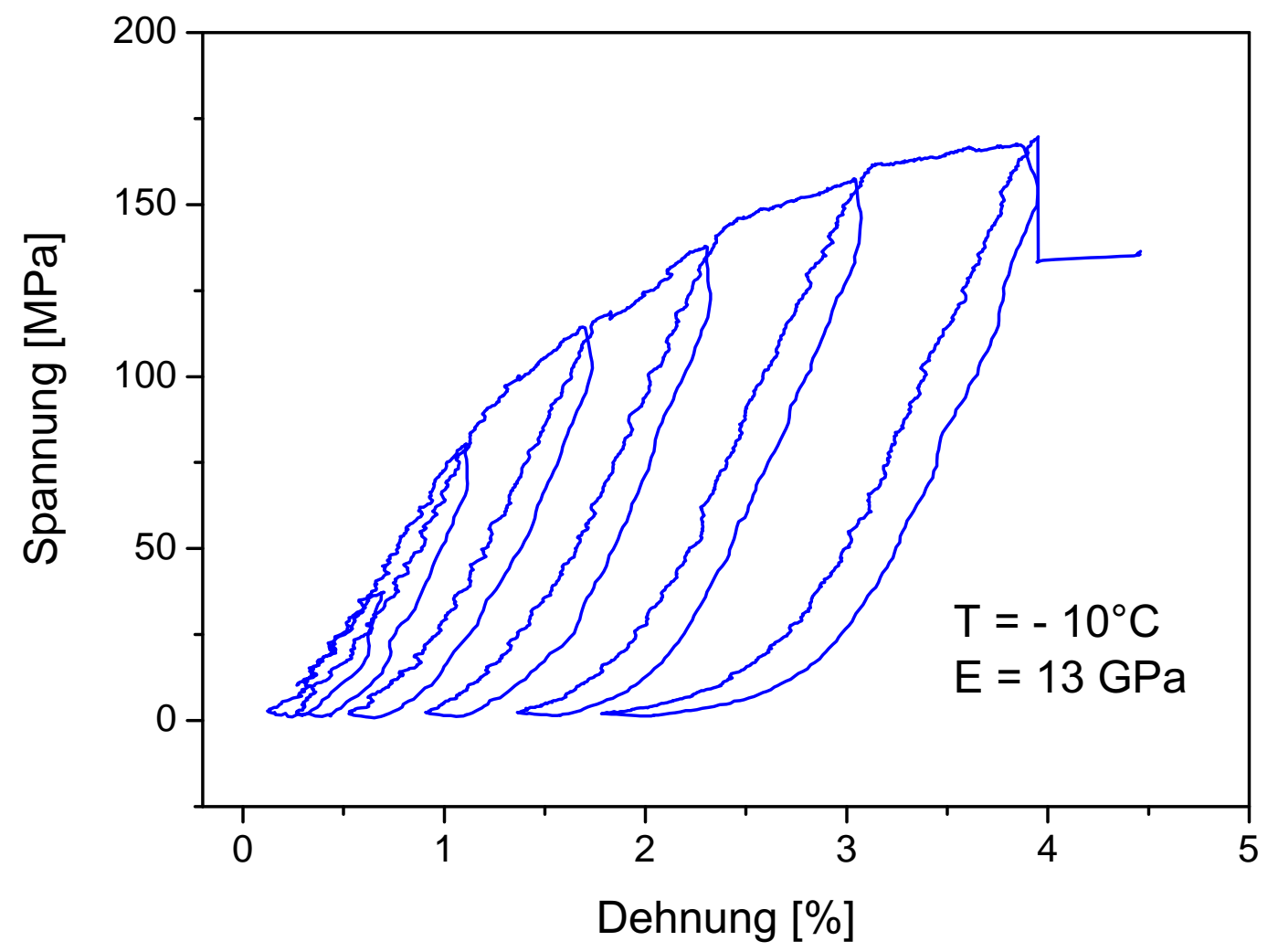

Abbildung 4.31: Im Martensit bleibt schon bei geringen Dehnungen ein plastischer Anteil bestehen und die Probe ist weicher als im Austenit. Beim Entlasten verdrillt sich die Probe. (Messung: C.Bechtold) 
Erhöhung des Magnetfeldes zum Teil unter der Aufösungsgrenze des Geräts, weshalb jeweils über fünf aufeinander folgende Messpunkte gemittelt wurde, um die scheinbare Stufenform auszugleichen. Ein negativer Wert für die Dehnung $\epsilon$ entspricht einer Kontraktion der Probe. In einem senkrecht zur Messrichtung angelegten Feld von 0,5 T zieht sich die martensitische Probe um 0,05\% zusammen. Wird das Magnetfeld reduziert, so dehnt sie sich wieder aus. Die Kontraktion $\epsilon$ der Probe hängt näherungsweise linear mit dem angelegten Feld zusammen, so dass eine Gerade mit der Steigung $\frac{\mathrm{d} \epsilon}{\mathrm{d} H} \approx-0,118 \% / \mathrm{T}$ angepasst werden kann. Die austenitische $\mathrm{Fe}_{70} \mathrm{Pd}_{30}$-Probe zeigt ein ähnliches Verhalten. Ihre Dehnung ist mit $0,09 \%$ bei $0,5 \mathrm{~T}$ etwas größer, so dass auch die angepasste Gerade eine etwas größere Steigung von $\frac{\mathrm{d} \epsilon}{\mathrm{d} H} \approx-0,186 \% / \mathrm{T}$ aufweist.

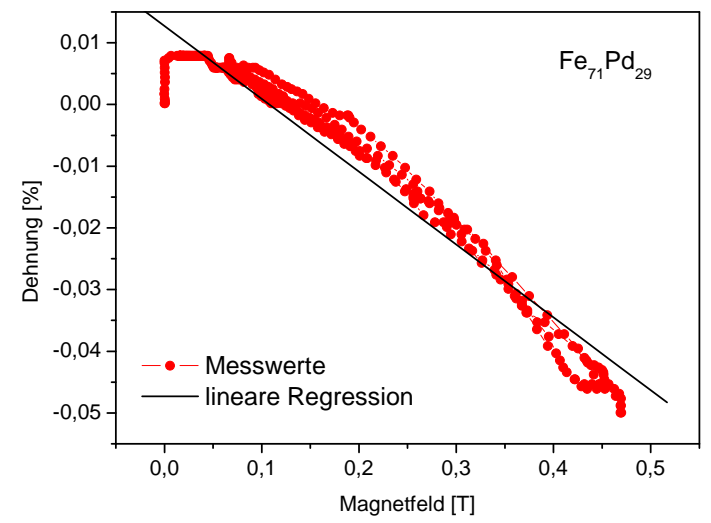

(a) Martensit

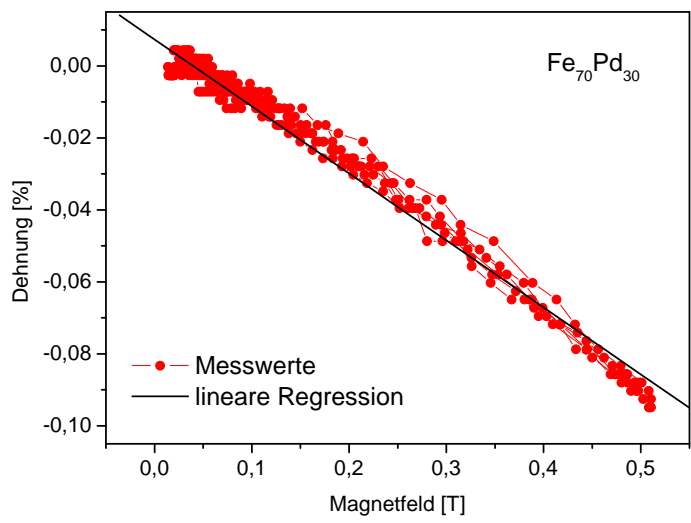

(b) Austenit

Abbildung 4.32: In einem senkrecht zur Messrichtung angelegten Feld zieht sich sowohl die martensitische als auch die austenitische Probe zusammen. (Messung: C.Bechtold) 


\section{Kapitel 5}

\section{Diskussion der Ergebnisse}

In diesem Teil der Arbeit sollen die in Kapitel 4 vorgestellten experimentellen Ergebnisse in einen gemeinsamen Zusammenhang gebracht und vor dem Hintergrund der etablierten und aktuellen Literatur diskutiert werden. Besonderes Augenmerk soll dabei auf die Frage gelegt werden, welche Faktoren die Bildung und Stabilität der martensitischen Phase und den martensitischen Phasenübergang beeinflussen. In Abschnitt 4.2 wurde beobachtet, dass neben der Zusammensetzung die Präparation der Proben insbesondere die Glühbehandlung einen entscheidenden Einfluss darauf hat, ob die Proben entmischen und welche Phase sich bei Zimmertemperatur einstellt. Dabei fällt auf, dass jene Proben, die bei besonders tiefen Temperaturen einen martensitische Übergang von der fcc- zur fct-Phase durchlaufen, eine besonders geringe Neigung zum Entmischen zeigen. Die Stabilität der fcc-Phase gegen Entmischen oder die Bildung der bcc-Phase und der martensitische Phasenübergang scheinen also eng miteinander verknüpft zu sein, so dass es schwierig ist, diese beiden Phänomene vollkommen getrennt voneinander zu diskutieren. Der Übersicht halber soll dennoch zunächst untersucht werden, welche Faktoren die Dekomposition der Legierung beeinflussen und welche Präparationsbedingungen die Bildung der fcc oder fct-Phase begünstigen. Nach einer ausführlichen Diskussion der mechanischen Eigenschaften der Splats im Austenit, im Martensit und während des Phasenüberganges sollen die charakteristischen Änderungen von Widerstand und Magnetisierung während des Phasenüberganges interpretiert werden. Abschließend wird in einer zusammenfassenden Diskussion der Einfluss der Präparation auf den martensitischen Phasenübergang erläutert.

\subsection{Stabilität gegen Entmischen}

Aus Abschnitt 2.7 ist bekannt, dass die binäre Eisen-Palladium-Legierung im thermodynamischen Gleichgewicht unterhalb von ca. $760{ }^{\circ} \mathrm{C}$ in eine geordnete Phase der Zusammensetzung $\mathrm{Fe}_{50} \mathrm{Pd}_{50}$ und $\alpha$-Eisen mit einer sehr geringen Menge Palladium entmischt [19]. Ein ähnliches Verhalten ist für ternäre Legierungen mit geringem Kupfergehalt zu erwarten und wird tatsächlich bei einem Teil der untersuchten Proben beobachtet. Dabei ist ein klarer Einfluss der Abkühlgeschwindigkeit zu erkennen: Je schneller die Proben abgeschreckt werden, desto weniger Ausscheidungen von $\alpha$-Eisen und $\mathrm{Fe}_{50} \mathrm{Pd}_{50}$ finden sich in der Mischkristallmatrix. Dies ist nicht überraschend, da das 
Entmischen ein diffusiver Prozess ist und die Diffusionsgeschwindigkeit bekanntermaßen stark von der Temperatur und der treibenden Kraft abhängt [27]. Bei der Bildung makroskopischer Ausscheidungen muss Material über Entfernungen bewegt werden, die weit größer sind als interatomare Abstände. Solange die Probentemperatur nur wenig unter der Mischbarkeitsgrenze liegt, ist zwar die Beweglichkeit der Atome groß, die treibende Kraft zum Entmischen ist jedoch gering, so dass kaum Phasenseparation stattfindet. Bei geringen Temperaturen hingegen ist zwar die treibende Kraft groß, die Beweglichkeit der Atome jedoch gering, so dass der Materietransport kinetisch gehemmt ist. Die größten Ausscheidungen werden gefunden, wenn das Material längere Zeit bei mittleren Temperaturen gehalten wurde. Dies ist zum Beispiel der Fall, wenn die Proben langsam im Ofen abkühlen.

Doch auch in rasch abgekühlten Proben sind Ausscheidungen zu finden, wenn die Glühtemperatur unterhalb der Mischbarkeitsgrenze von $760{ }^{\circ} \mathrm{C}$ liegt. Die Röntgendiffraktogramme in Abbildung 4.17 zeigen, dass in Proben, die bei Temperaturen von $700{ }^{\circ} \mathrm{C}$ oder weniger geglüht wurden, Eisenausscheidungen auftreten. Wegen der Temperaturabhängigkeit der Diffusionsgeschwindigkeit ist der Eisen-Peak einer bei $700{ }^{\circ} \mathrm{C}$ geglühten Probe stärker ausgeprägt und schärfer als bei einer bei $600^{\circ} \mathrm{C}$ geglühten Probe. Es haben sich also größere Ausscheidungen gebildet. Dies wird durch die Überprüfung der Probenzusammensetzung mit EDX an verschiedenen über die gesamte Fläche der Probe verteilten Stellen bestätigt, die einen mittleren Eisengehalt von 70,3 $\pm 1,0 \%$ in der bei $700{ }^{\circ} \mathrm{C}$ geglühten Probe ergibt. In anderen Proben, die bei verschiedenen Temperaturen geglüht wurden (auch bei $600^{\circ} \mathrm{C}$ ), lag die Schwankung des Eisengehalts unter $\pm 0,5 \%$. Der Eisengehalt der bei $700{ }^{\circ} \mathrm{C}$ geglühten Probe variiert also deutlich stärker als bei den anderen Proben. Beim Vergleich von Proben verschiedener Zusammensetzung, die der gleichen Wärmebehandlung unterzogen wurden, zeigt sich jedoch, dass nicht allein Glühtemperatur und Abkühlgeschwindigkeit das Ausscheidungsverhalten bestimmen. In einigen Proben werden auch bei hohen Glühtemperaturen und hohen Abkühlraten große Mengen an Ausscheidungen gefunden. Da die übrigen Präparationsparameter identisch sind, ist davon auszugehen, dass die Zusammensetzung der Proben einen starken Einfluss auf ihre Neigung zum Entmischen hat.

Aus der Literatur ist bekannt, dass neben dem Verhältnis der Atomradien und Elektronegativitäten die Valenzelektronenkonzentration und insbesondere die Füllung der $d$-Bänder einen großen Einfluss auf die Mischbarkeit von Metallen hat [82, 83]. Das Modell von Hume-Rothery bezieht sich jedoch auf das thermodynamische Gleichgewicht, ist auf Legierungen von Übergangsmetallen mit Hauptgruppen-Elementen beschränkt und geht nicht auf das Mischungsverhalten der Übergangsmetalle untereinander ein. Zudem sind die Variationen der Zusammensetzungen der untersuchten Proben zu klein, um das unterschiedliche Entmischungsverhalten anhand dieses Modells zu erklären.

Stattdessen bietet es sich an, die Ergebnisse aus Abschnitt 4.2 in Beziehung zu aktuellen Messungen von Hamann et al. zu setzen [25]. Hier wurden durch Sputterdeposition polykristalliner Schichten auf oxidierte Siliziumsubstrate Materialbibliotheken des ternären Eisen-Palladium-Kupfer-Systems hergestellt. Auffällig ist, dass in allen untersuchten Schichten, die nicht den martensitischen Phasenübergang durchlaufen, Ausscheidungen auftreten. Zwar gibt es Hinweise, dass die Neigung zum Entmischen in dünnen, auf Siliziumdioxid deponierten Schichten gegenüber Massivproben deutlich erhöht ist [66], die gleiche Tendenz wurde jedoch auch an den in dieser Arbeit unter- 
suchten Splats beobachtet: Proben, die den martensitischen Phasenübergang bei tiefen Temperaturen durchlaufen, zeigen eine geringere Neigung zum Entmischen.

Das bedeutet jedoch nicht, dass transformierende Proben grundsätzlich nicht entmischen. So wird bei unbehandelten binären Proben mit einem Palladiumgehalt von $29 \%$ ein martensitischer Phasenübergang beobachtet, bei einer anschließenden Glühbehandlung treten jedoch auch bei relativ hohen Abkühlraten Ausscheidungen auf. Das Auftreten der Transformation allein scheint also kein hinreichendes Kriterium für die Stabilität zu sein. Diese Proben befinden sich allerdings in einem Konzentrationsbereich, in dem nach dem Phasendiagramm von Cui et al. die bct-Phase und damit keine Transformation zu erwarten wäre [6]. Somit ist es wahrscheinlich, dass eine gemeinsame Ursache für Stabilität und martensitische Transformation existiert, die mit der Zusammensetzung der Legierung verknüpft ist, während der martensitische Phasenübergang auch durch andere Faktoren beeinflusst wird, die in Abschnitt 5.4.3 diskutiert werden sollen.

Um zu bestimmen, welche Einflüsse für die Stabilität der Legierung verantwortlich sind, bietet es sich wiederum an, das ternäre System zu betrachten, da hier verschiedene Parameter wie Eisengehalt und Valenzelektronenkonzentration unabhängig voneinander variiert werden können. Beim Betrachten des ternären Phasendiagramms von Hamann et al. ,das in Abbildung 5.1 skizziert ist, fällt auf, dass alle transformierenden Proben ohne Ausscheidungen in einem schmalen Zusammensetzungsbereich liegen, der durch zwei senkrechte Geraden begrenzt wird [25]. Entlang einer Geraden werden Eisen und Palladium gleichermaßen durch Kupfer ersetzt. Eine Gerade enthält alle Punkte mit $\mathrm{Fe}_{71-a} \mathrm{Pd}_{29-a} \mathrm{Cu}_{2 a}$, die andere alle Punkte mit $\mathrm{Fe}_{73-a} \mathrm{Pd}_{27-a} \mathrm{Cu}_{2 a}(0<a<3)$. Auf den ersten Blick passt dies nicht mit den Ergebnissen aus Abschnitt 4.2 zusammen, da stabile Phasen in Splats bei niedrigerem Eisengehalt gefunden wurden. Es ist jedoch möglich, dass in dünnen Schichten die Grenze des Stabilitätsbereichs zu höherem Eisengehalt verschoben ist. So ist bekannt, dass in binären epitaktischen Filmen die Grenze zwischen der bcc- und der transformierenden fct-Phase im Vergleich zu massiven Einkristallen um 2 Atomprozent zu höherem Eisengehalt verschoben ist [6, 84]. Da Transformation und Stabilität eng verknüpft sind, wäre damit auch eine Verschiebung der Grenze des Stabilitätsbereiches nicht verwunderlich. Ein Vergleich der Daten für Massivmaterial von Cui et al. mit dünnen Schichten von Buschbeck et al. und der Extrapolation der Daten von Hamann et al. zu binären Schichten bestätigt dies [6, 25, 84]. Des Weiteren besteht die Möglichkeit, dass die von Hamann et al. angegebenen Eisenkonzentrationen geringfügig höher sind als der tatsächliche Eisengehalt der Schichten. Der Grund hierfür ist die Bildung einer dünnen Eisenoxidschicht an der Grenzfläche zum Substrat, die dazu führt, dass die Schichten geringfügig an Eisen verarmen, was bei einer Prüfung mit EDX nicht nachweisbar ist. Dieser Effekt sollte jedoch nicht zu einer Abweichung von 2 Atomprozent über das gesamte Volumen der Schicht führen.

Damit lässt sich das Stabilitätsverhalten aus Abschnitt 4.2 erklären. Die Proben mit Zusammensetzungen von $\mathrm{Fe}_{70} \mathrm{Pd}_{30}, \mathrm{Fe}_{71} \mathrm{Pd}_{29}$ und $\mathrm{Fe}_{68,4} \mathrm{Pd}_{29,3} \mathrm{Cu}_{2,3}$ liegen alle in einem Bereich, in dem nach dem Diagramm von Hamann et al. Ausscheidungen zu erwarten wären [25]. Die Probe mit der Zusammensetzung $\mathrm{Fe}_{70} \mathrm{Pd}_{23,8} \mathrm{Cu}_{6,2}$ hingegen dürfte nicht entmischen. Verschiebt man jedoch den Stabilitätsbereich um 2 Atomprozent zu niedrigeren Eisenwerten, so enthält er die drei erstgenannten Proben, während die Probe mit $6,2 \%$ Kupfer deutlich außerhalb liegt. 


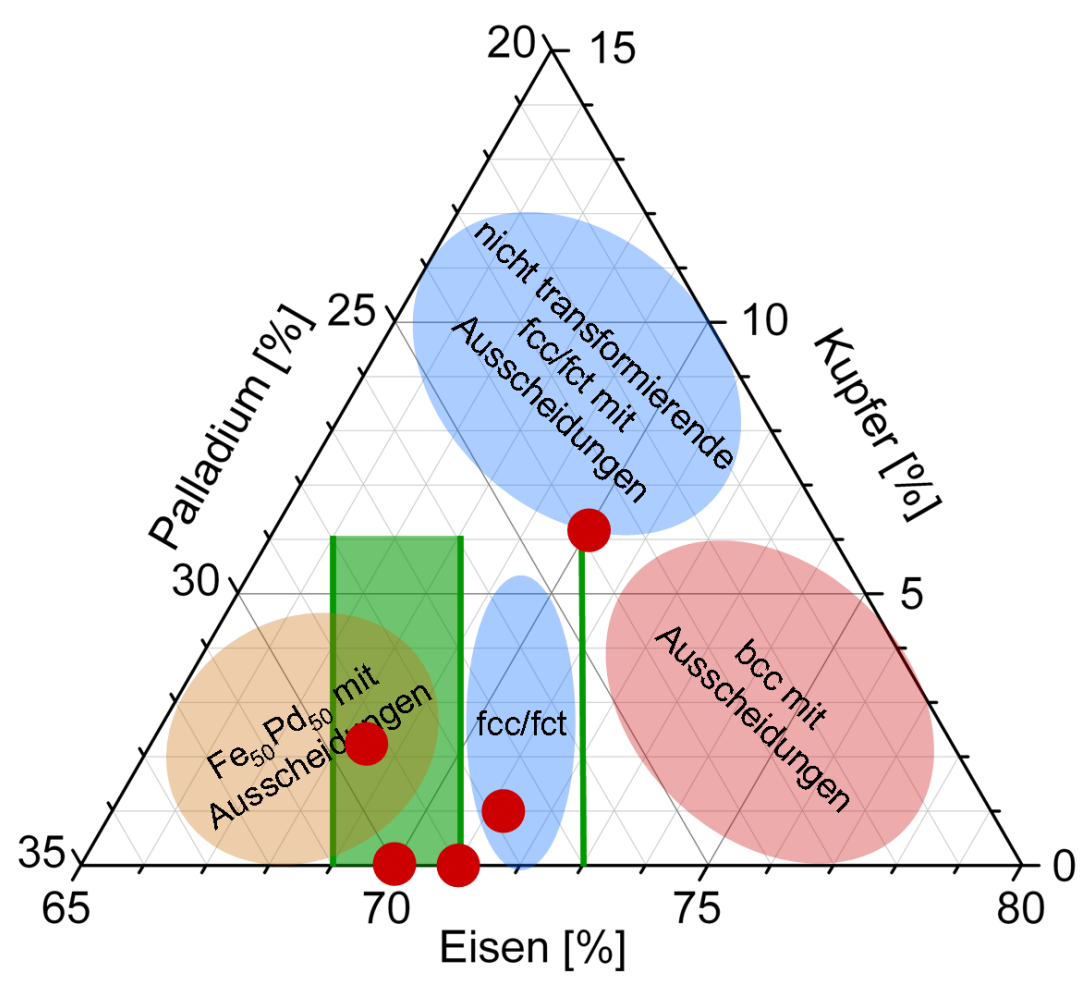

\begin{abstract}
Abbildung 5.1: Nach dem ternären Phasendiagramm der gesputterten Schichten von Hamann et al. aus [25] liegen alle Proben ohne Ausscheidungen zwischen den grünen Linien, die den Bereich zwischen $\mathrm{Fe}_{71-a} \mathrm{Pd}_{29-a} \mathrm{Cu}_{2 a}$ und $\mathrm{Fe}_{73-a} \mathrm{Pd}_{27-a} \mathrm{Cu}_{2 a}$ markieren. Für Massivmaterial ist dieses Gebiet in den grün schattierten Bereich erweitert [6, 84]. Die roten Kreise repräsentieren die in dieser Arbeit untersuchten Proben.
\end{abstract}

Die Frage, warum gerade Proben aus dem oben genannten Zusammensetzungsbereich stabil sind, bleibt jedoch unbeantwortet. Es können lediglich einige Faktoren als Ursache für die Stabilität ausgeschlossen werden. Zunächst einmal kann die Notwendigkeit einer bestimmten Elektronenkonzentration ausgeschlossen werden, da in dem Stabilitätsbereich zwischen den oben genannten Geraden verschiedene Elektronenkonzentrationen gefunden werden. Auch ein bestimmter Eisengehalt oder ein bestimmtes Verhältnis von Eisen- und Palladiumkonzentration scheiden aus.

Die Verschiebung des Stabilitätsbereichs für dünne Schichten führt wieder zu präparativen und mikrostrukturellen Einflüssen als Ursache für die Stabilität zurück. Die Schichten von Hamann et al. wurden nach der Deposition für eine Stunde bei $850{ }^{\circ} \mathrm{C}$ geglüht und anschließend mit einer moderaten Geschwindigkeit von $15 \mathrm{~K} / \mathrm{s}$ abgekühlt, so dass diese Proben mit den an Luft abgekühlten Splats vergleichbar sein sollten. Die Verschiebung des Stabilitätsbereichs zeigt jedoch, dass offenbar ein Unterschied zwischen den Splats und dünnen Schichten besteht. Als möglich Ursachen müssen hier Oberflächensegregation und Korngrenzensegregation von Eisen in Betracht gezogen werden. Dünne Schichten weisen ein größeres Oberfläche-zu-Volumen-Verhältnis auf als Splats. Da die Korngröße dünner Schichten in der Regel die Schichtdicke nicht übersteigt, ist außerdem davon auszugehen, dass die Körner der Schichten kleiner sind als die der Splats, so dass die Segregation von Eisen an Korngrenzen oder Oberflächen zu einer stärkeren Verarmung an Eisen führen würde. Damit wäre der tatsächliche Eisengehalt innerhalb 
der Körner geringer als angegeben. Zudem könnten Grenzflächenspannungen einen entscheidenden Einfluss haben. Kühnemund et al. konnten bei starker Wechselwirkung mit dem Substrat einen deutlichen Einfluss auf das Separationsverhalten nachweisen [66]. Des Weiteren ist in dünnen Schichten immer mit einer intrinsischen Verspannung zu rechnen, und dass Verzerrungen des Kristallgitters die thermodynamischen Eigenschaften von Mischkristallen beeinflussen können, muss ebenfalls berücksichtigt werden [85].

Zusammenfassend lässt sich also festhalten, dass die Neigung der Proben zum Entmischen sowohl durch die Präparation als auch durch ihre Zusammensetzung beeinflusst wird. So begünstigt Glühen bei Temperaturen unter $760^{\circ} \mathrm{C}$ oder langsames Abkühlen bei allen untersuchten Zusammensetzungen die Bildung von Ausscheidungen. Bei hinreichend hohen Glühtemperaturen und hinreichend schnellem Abkühlen sind dünne Schichten mit einer Zusammensetzung zwischen $\mathrm{Fe}_{71-a} \mathrm{Pd}_{29-a} \mathrm{Cu}_{2 a}$ und $\mathrm{Fe}_{73-a} \mathrm{Pd}_{27-a} \mathrm{Cu}_{2 a}$ stabil gegen Entmischen. Für Splats ist dieser Bereich um $2 \% \mathrm{zu}$ niedrigerem Eisengehalt erweitert. Er enthält alle Proben, die den martensitischen Phasenübergang durchlaufen. Es besteht also ein Zusammenhang zwischen der Transformation und der Stabilität gegen Entmischen.

\subsection{Phasen bei Zimmertemperatur}

Die Ergebnisse aus Abschnitt 4.2 zeigen, dass im unbehandelten Zustand direkt nach dem Splatquenching fast alle untersuchten binären Proben bei Zimmertemperatur in der fcc-Phase vorliegen. Lediglich Proben mit einem Palladiumgehalt um $29 \%$ sind martensitisch (fct) und unterhalb von $28 \%$ tritt die bcc-Phase auf. Für hohen Palladiumgehalt ab $30 \%$ stimmen diese Ergebnisse gut mit denen von Cui et al. und Matsui et al. , die in Abbildung 2.9 gezeigt sind, überein [3, 6]. Bei niedrigerem Palladiumgehalt zeigt sich jedoch eine Diskrepanz: In einkristallinem Material ist nach Cui et al. bereits bei einem Palladiumgehalt unter $29 \%$ die bcc-/bct-Phase zu erwarten. An Polykristallen hingegen beobachten Matsui et al. bei $29 \%$ Palladium die fct-Phase. Abhängig von der Kühlrate wurde zudem eine Koexistenz der fct- und bcc-Phase bis zu einem Palladiumgehalt von $27 \%$ beobachtet [3]. Die Autoren bieten jedoch keine Erklärung für diese Kühlratenabhängigkeit an.

Eine ähnliche Kühlratenabhängigkeit ist in den Ergebnissen aus Abschnitt $4.2 \mathrm{zu}$ sehen. Proben, die im unbehandelten Zustand in der fct-Phase vorliegen, transformieren während einer Glühbehandlung sukzessive in die bct- bzw. bcc-Phase. Da beide Phasen nacheinander an derselben Probe beobachtet werden, können Einflüsse der über die Probe gemittelten Zusammensetzung ausgeschlossen werden. Auch starke lokale Fluktuationen des Palladiumgehalts, die sich beim Glühen aufgrund der Tendenz zum Entmischen bilden könnten, kommen nicht in Betracht. In den Diffraktogrammen in Abschnitt 4.2.2 hat sich gezeigt, dass präferentiell Eisen ausgeschieden wird. Eine Verarmung an Eisen würde aber zu einem Anstieg des Palladiumgehalts und damit zur Stabilisierung der fcc-Phase führen.

Stattdessen liegt es nahe, die Ursache in der Mikrostruktur der Probe zu suchen, die sich während einer Glühbehandlung nachweislich ändert. Vor der Glühbehandlung ist die Mikrostruktur der Proben wegen der extremen Abkühlgeschwindigkeit beim Splatquenching sehr feinkristallin mit vielen Defekten und starker Verzerrung des Kristall- 
gitters. Dies ist zum einen an der Form und Breite der Röntgenreflexe in Abbildung 4.7 und zum anderen an der Säulenbreite der REM- und TEM-Bilder in Abschnitt 4.1 zu erkennen. Schon während einer kurzen Glühbehandlung von 15 Minuten steigt diese Säulenbreite signifikant an. Darüber hinaus können Spannungen abgebaut werden und Defekte ausheilen [27]. Damit liegt die Vermutung nahe, dass eine defektreiche Mikrostruktur die fct-Phase gegenüber der bct-Phase begünstigt.

Der Grund dafür, dass bei einer defektreichen Mikrostruktur bei Zimmertemperatur die fct-Phase anstelle der bct-Phase auftritt, kann nicht einfach darin liegen, dass der Phasenübergang von der fct- in die bct Phase kinetisch unterdrückt ist. Denn dieser Phasenübergang ist diffusionlos und damit in weit geringerem Maße temperaturabhängig als beispielsweise die Separation in die equiatomare Phase und $\alpha$-Eisen. Zudem findet der Übergang von der fct- in die fcc-Phase beim Heizen durchaus statt und ist mehrfach reproduzierbar. Des Weiteren konnte beim Abkühlen auf Stickstofftemperatur ein irreversibler Übergang in die bct-Phase nachgewiesen werden; diese Transformation ist also grundsätzlich auch möglich, findet jedoch erst weit unterhalb von Zimmertemperatur statt. Es ist eine sehr große Unterkühlung $\Delta T$ gegenüber der Gleichgewichtstemperatur $T_{0}$ notwendig, die nach dem Phasendiagramm von Cui et al. weit oberhalb von Zimmertemperatur liegt [6]. Nach Abschnitt 2.1 muss also beim Phasenübergang eine große nicht-chemische freie Enthalpie aufgebracht werden. Nach Abbildung 2.2 ist ein Absenken der Martensitstarttemperatur mit dem Auftreten großer innerer Reibung verknüpft. Dann müsste allerdings auch der Übergang von der fcc- in die fct-Phase zu tieferen Temperaturen verschoben sein. Dieser tritt jedoch deutlich oberhalb von Zimmertemperatur auf.

Stattdessen muss berücksichtigt werden, dass in polykristallinen verspannten Proben schon vor Beginn des Phasenübergangs eine signifikante elastischer Energie gespeichert ist. Nach Gleichung 2.2 kann die Übergangstemperatur von der fcc- in die fct-Phase auch durch eine externe elastische Verzerrung verschoben werden, da diese in der fctPhase durch Ausrichten der Martensitvarianten besser kompensiert werden kann. Die bcc-Phase hingegen ist nicht tetragonal verzerrt, und die Verzerrung der bct-Phase ist sehr viel geringer als die der fct-Phase, so dass ein Spannungsabbau hier deutlich schwieriger ist. Im verzerrten Material ist also zu erwarten, dass sich die Übergangstemperatur von der fct- in die bcc-Phase zu tiefen Temperaturen verschiebt. Dies wird durch die experimentellen Daten bestätigt: Die von Cui et al. untersuchten Einkristalle haben die höchste Übergangstemperatur [6], gefolgt von den polykristallinen Proben aus den Arbeiten von Matsui et al. [3] und den geglühten Splats, während die niedrigsten Übergangstemperaturen in unbehandelten Splats gefunden werden.

Dass in verzerrten Proben die fct-Phase gegenüber der bcc-Phase begünstigt ist, lässt sich auch anhand der flachen Energielandschaft entlang des Bain-Pfades verstehen, die von Buschbeck et al. berechnet wurde und in Abbildung $2.10 \mathrm{zu}$ sehen ist [51]. In der Umgebung des fct-Minimums ist die Energielandschaft noch flacher als im bccMinimum, so dass für die gleiche elastische Verzerrung eine geringere Energie aufgewandt werden muss. Bei der Interpretation von Abbildung 2.10 ist jedoch zweierlei zu beachten: Zum einen wurden die Berechnungen für einen Palladiumgehalt von $30 \%$ am absoluten Temperaturnullpunkt durchgeführt. Bei endlichen Temperaturen ist davon auszugehen, dass sich die Enthalpien durch den Einfluss der Entropie leicht gegeneinander verschieben. Des Weiteren kann ein leicht reduzierter Palladiumgehalt die Lagen 
der Energieminima beeinflussen. Zum anderen haben Buschbeck et al. zwar die Energien für verschiedene tetragonale Verzerrungen berechnet, die Atome wurden jedoch auf ihren Positionen fixiert. In einem realen Kristall hingegen kann das Gitter relaxieren, um unter Umständen eine günstigere Konfiguration anzunehmen. Dies wurde in aktuellen Berechnungen von Gruner et al. berücksichtigt, die Relaxationsprozesse erlauben und tatsächlich die bcc-Phase als Grundzustand bestätigen [54]. Hier zeigt sich eine gute Übereinstimmung zwischen den theoretischen Vorhersagen der Simulationen und den hier erhaltenen experimentellen Ergebnissen: In einem starren Gitter tritt die fct-Phase auf. Werden jedoch, beispielsweise durch eine Glühbehandlung, Relaxationsprozesse ermöglicht, stellt sich die bcc-Phase ein.

Zur relativen Stabilität der Phasen in Eisen-Palladium-Kupfer-Legierungen existieren noch keine Berechnungen. Generell wäre wegen des geringen Radius der Kupferatome ein geringeres Volumen der Einheitszelle zu erwarten. Im Rahmen der Messgenauigkeit wird dies jedoch nicht beobachtet. Die Masse der Kupferatome liegt zwischen der von Eisen- und Palladiumatomen. Abhängig davon, welche Atomsorte durch Kupfer ersetzt wird, kann damit das Phononenspektrum zu höheren oder niedrigeren Frequenzen verschoben werden. Da der martensitische Phasenübergang eng mit einem Aufweichen bestimmter Phononenmoden verknüpft ist, kann ein Einfluss auf die Übergangstemperatur und damit auf die bei Zimmertemperatur vorliegende Phase erwartet werden. Eine direkte Verbindung zwischen dem Kupfergehalt und der Übergangstemperatur ist den vorliegenden Daten jedoch nicht zu entnehmen.

Allerdings existiert ein indirekter Einfluss des Kupfergehalts über die Valenzelektronenkonzentration. Den experimentellen Daten von Hamann et al. in Abbildung 5.1 ist zu entnehmen, dass außerhalb des Stabilitätsbereichs der fct-Phase die Grenze zwischen der fct- und der bcc-Phase etwa bei einer Valenzelektronenkonzentration von $e / a=8,59$ verläuft [25]. Dieses Verhalten wird von den geglühten und in Wasser abgeschreckten Splats bestätigt. Proben mit mehr als 8,59 Valenzelektronen pro Atom liegen, sofern sie nicht entmischen, in der fcc- oder fct-Phase vor, während Proben mit einer geringeren Valenzelektronenkonzentration zumindest zum Teil in der bcc- bzw. bct-Phase vorliegen (siehe Tabelle 5.1). Die Zugabe von Kupfer ermöglicht es also, auch bei einem Palladiumgehalt unter $30 \%$ die fcc-Phase zu bewahren bzw. bei einem festen Eisengehalt die Valenzelektronenkonzentration zu erhöhen.

Im Hinblick auf technische Anwendungen, beispielsweise des Formgedächtniseffekts, ist diese Möglichkeit, bei fester Valenzelektronenkonzentration den Eisengehalt zu erhöhen, höchst interessant. Die Daten von Hamann et al. weisen darauf hin, dass die

\begin{tabular}{l|c|c|r} 
Zusammensetzung & $e / a$ & Phase & $M_{s}$ \\
\hline $\mathrm{Fe}_{70,0} \mathrm{Pd}_{23,8} \mathrm{Cu}_{6,2}$ & 8,66 & fct & $33^{\circ} \mathrm{C}$ \\
$\mathrm{Fe}_{68,4} \mathrm{Pd}_{29,3} \mathrm{Cu}_{2,3}$ & 8,64 & fcc & $-35^{\circ} \mathrm{C}$ \\
$\mathrm{Fe}_{70,0} \mathrm{Pd}_{30,0}$ & 8,60 & fcc & $13^{\circ} \mathrm{C}$ \\
$\mathrm{Fe}_{71,2} \mathrm{Pd}_{27,8} \mathrm{Cu}_{1,0}$ & 8,59 & fct + bct & $77^{\circ} \mathrm{C}$ \\
$\mathrm{Fe}_{71,0} \mathrm{Pd}_{29,0}$ & 8,58 & fct + bct & $52^{\circ} \mathrm{C}$
\end{tabular}

Tabelle 5.1: Bei einer Valenzelektronenkonzentration unter 8,59 tritt in geglühten Proben die bcc- oder bct-Phase auf. 


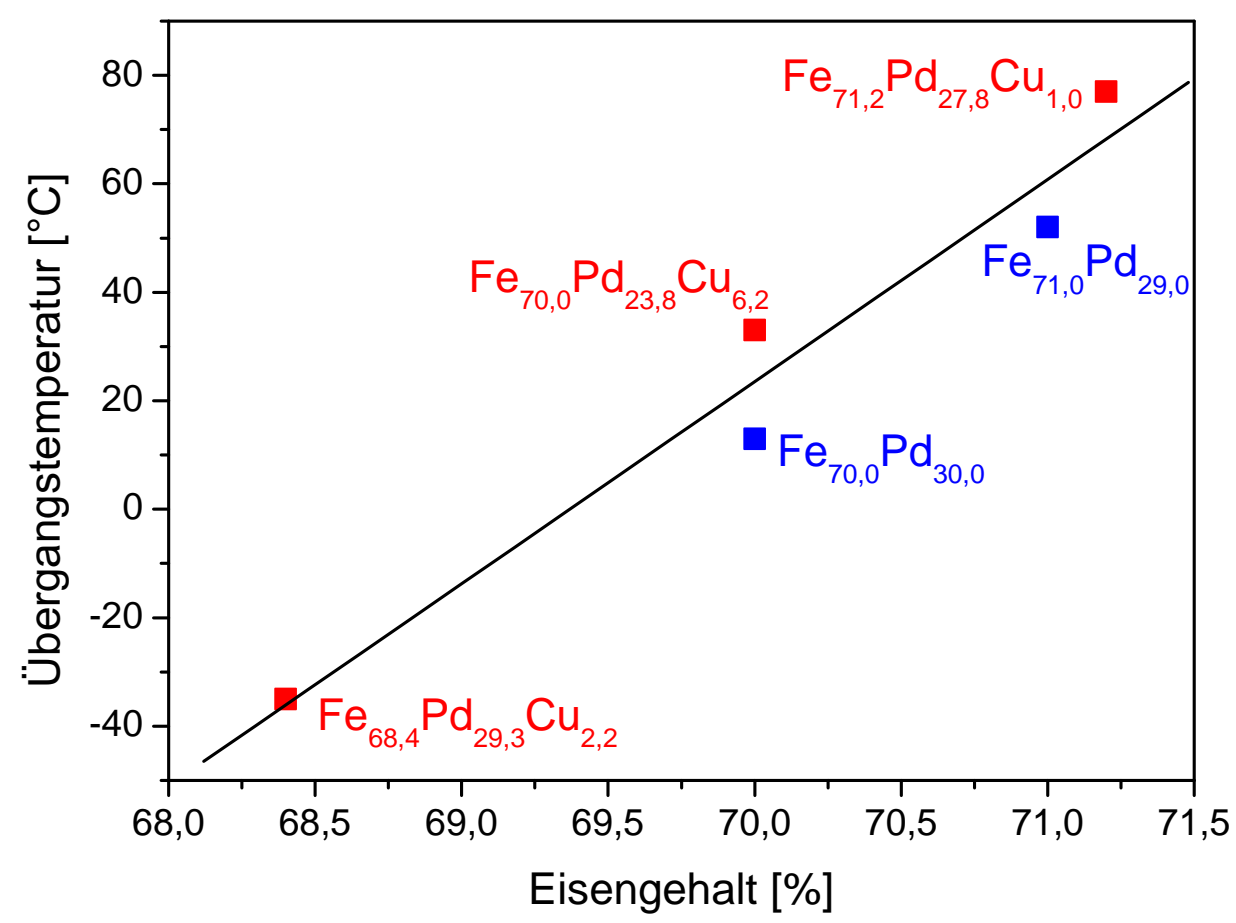

Abbildung 5.2: Die Übergangstemperatur steigt linear mit dem Eisengehalt der Proben an.

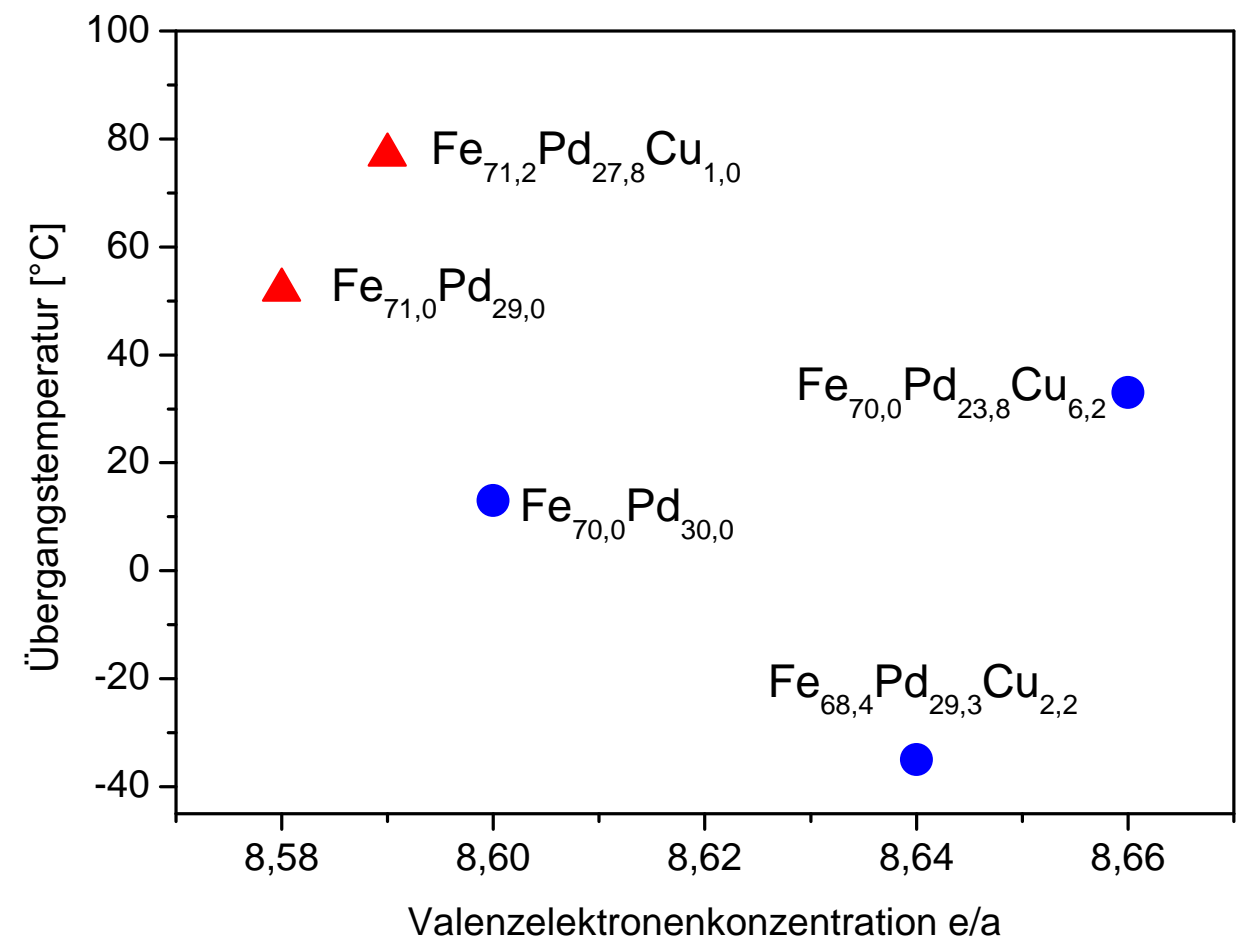

Abbildung 5.3: Proben mit mehr als 8,59 Valenzelektronen pro Atom liegen nach dem Glühen in der fcc-Phase (blaue Kreise) vor. Bei einer niedrigeren Valenzelektronenkonzentration tritt auch die bct-Phase (rote Dreiecke) auf. Eine Korrelation mit der Übergangstemperatur ist jedoch nicht zu erkennen. 
Übergangstemperatur sowohl vom Eisengehalt als auch von der Elektronenkonzentration abhängt [25]. Der Anstieg der Übergangstemperatur mit dem Eisengehalt wird durch die Ergebnisse der Splats (Abbildung 5.2) bestätigt. Eine Abhängigkeit von der Elektronenkonzentration ist jedoch nicht zu erkennen (siehe Abbildung 5.3). Damit ist es möglich, durch Einstellen einer geeigneten Valenzelektronenkonzentration die fccbzw. fct-Phase zu stabilisieren und gleichzeitig durch Erhöhen des Eisengehalts die Übergangstemperatur anzuheben. Die Daten in Tabelle 5.1 und Abbildung 5.3 zeigen jedoch auch, dass die höchsten Übergangstemperaturen in Proben auftreten, die zum Teil in der bct-Phase vorliegen. An der Phasengrenzfläche wird das Kristallgitter verzerrt. Die dabei entstehenden Spannungen können nach Gleichung 2.2 ebenfalls die Übergangstemperatur erhöhen.

Damit konnte in diesem Abschnitt gezeigt werden, dass nicht nur die Zusammensetzung bestimmt, welche Phase sich bei Zimmertemperatur einstellt. Auch die Mikrostruktur der Proben ist von entscheidender Bedeutung: Eine hohe Defektdichte begünstigt die Bildung der fct-Phase. Erhält das Kristallgitter die Möglichkeit, Defekte abzubauen und zu relaxieren, so transformieren Proben mit einer Valenzelektronenkonzentration unter 8,59 Elektronen pro Atom in die bcc-Struktur. Bei einer höheren Valenzelektronenkonzentration tritt die fct-Phase auch im relaxierten Zustand auf. Die Transformationstemperatur von der fct- in die fcc-Phase hängt direkt vom Eisengehalt der Proben ab. Die Zugabe von Kupfer ermöglicht es, bei fester Elektronenkonzentration den Eisengehalt und damit die Übergangstemperatur zu erhöhen.

\subsection{Mechanische Eigenschaften}

Für eine genaue theoretische Vorhersage der Zusammenhänge zwischen Eisengehalt, Valenzelektronenkonzentration und Kristallstruktur müssen in Legierungen mit Übergangsmetallen auch die $d$-Bänder und deren Füllung berücksichtigt werden, die das magnetische Verhalten des Materials bestimmen. Über Magnetovolumeninstabilitäten sind diese mit den atomaren Abständen und der Kristallstruktur verknüpft, so dass mit einfachen Modellen keine Vorhersagen mehr getroffen werden können. Wie schwierig derartige Berechnungen sind, zeigen die Diskrepanzen zwischen den Ergebnissen von Buschbeck et al. [51] und Gruner et al. [86]. Diese Schwierigkeiten liegen zum Teil darin begründet, dass die Energieunterschiede zwischen den verschiedenen Strukturen sehr klein sind. Doch gerade die Tatsache, dass sich die Energien der verschiedenen Strukturen kaum unterscheiden, ermöglicht das interessante Verhalten des Materials unter mechanischer Belastung.

Wie klein die Energiedifferenzen zwischen den verschiedenen Strukturen sind, zeigt sich beispielsweise in den Ergebnissen aus Abschnitt 4.2.4 anhand der Tatsache, dass die Kristallstruktur einer Probe bei konstanter Temperatur durch die massive mechanische Verformung beim Walzen irreversibel verändert werden kann. Die rücktreibende Kraft, um die ursprüngliche Struktur wieder anzunehmen, ist zu gering, um die transformationbedingten Reibungsverluste und die durch die mechanische Verformung verursachten Verzerrungen zu überwinden. Doch auch bei weitestgehend elastischer Verformung ist der Einfluss der flachen Energielandschaft nicht zu übersehen. 
Im nächsten Abschnitt soll daher zunächst das elastische und plastische Verhalten der Splats bei uniaxialem Zug interpretiert werden. Anschließend wird die durch ein Magnetfeld induzierte Dehnung analysiert. Im darauffolgenden Abschnitt wird das Aufweichen des Kristallgitters während des Phasenüberganges diskutiert, das in dem Vibrating-Reed-Experiment beobachtet wurde, und die die daraus gewonnenen Elastizitätsmoduli mit denen der Zugversuche verglichen. Abschließend werden die Ursachen für den Anstieg der Dämpfung nach dem Phasenübergang erörtert.

\subsubsection{Spannungsinduzierte Dehnung}

Die Veränderung der Kristallstruktur beim Dehnen in Abbildung 4.15 zeigt deutlich, dass die Energieunterschiede zwischen den verschiedenen Phasen sehr gering sind. Im Zusammenhang mit den Spannungs-Dehnungskurven in Abbildung 4.29 veranschaulicht sie direkt den Energieverlauf einer uniaxialen Verzerrung entlang des Bainpfades. Beim uniaxialen Dehnen von isotropem Material würde man eine Vergößerung der Atomabstände in Zugrichtung und eine Verkleinerung senkrecht dazu erwarten. Da die Röntgenstrahlung senkrecht zur Zugrichtung einfällt, müssten sich alle Peaks zu größeren Winkeln verschieben [87]. Abbildung 4.15 zeigt jedoch ein anderes Verhalten. Der fcc/fct(111)-Peak verändert seine Position nicht, der Abstand der (111)-Ebenen und damit die Länge der Raumdiagonalen senkrecht zur Zugrichtung bleibt also konstant. Der (200)-Peak hingegen verschwindet, die Kohärenzlänge in (200)-Richtung ist also deutlich reduziert, wie es in stark verzwillingtem Martensit beobachtet wird. Intuitiv würde man erwarten, dass sich bei der Bildung von spannungsinduziertem Martensit die Martensitvarianten mit ihrer langen Achse in Dehnungsrichtung orientieren und der fcc(200)-Peak zur fct(200)-Position wandert. Der fct-Martensit hat jedoch zwei lange Achsen. Auch wenn eine davon in Dehnungsrichtung ausgerichtet wird, bleibt die Möglichkeit der Orientierung der anderen langen Achse senkrecht zur Probenoberfläche.

Bei uniaxialer Dehnung wandert die Struktur der Proben also entlang des Bainpfades von der fcc- zu einer stark verzwillingten fct-Struktur. Entsprechend der flachen Energielandschaft ist hierfür nur eine geringe Energie nötig, wie der niedrige Elastizitätsmodul von nur $25 \mathrm{GPa}$ zeigt, der aus der Spannungs-Dehnungskurve einer austenitischen Probe in Abbildung 4.30 gewonnen wurde. Bei weiterer Dehnung richten sich die Martensitvarianten zum Teil aus, so dass sie der Spannung maximal nachgeben. Gleichzeitig setzen plastische Prozesse ein, die zu einer irreversiblen Dehnung und schließlich zum Reißen des Materials führen. Bei diesen plastischen Prozessen kann es sich nicht um die Reorientierung der Varianten des spannungsinduzierten Martensits handeln, da das Material beim Entlasten zurück in die austenitische Phase transformiert. Dabei müsste sich die durch die Reorientierung verursachte Dehnung zurückbilden.

Kato et al. beobachten in Zugversuchen an Polykristallen der Zusammensetzung $\mathrm{Fe}_{30,5} \mathrm{Pd}_{69,5}$ ein sehr ähnliches Spannungs-Dehnungsverhalten [50]: Zunächst steigt die Spannung steil und nahezu linear mit der Dehnung an, bei höheren Spannungen über $150 \mathrm{MPa}$ flacht die Kurve jedoch deutlich ab. Auf Lichtmikroskop-Bildern der gedehnten Proben ist dabei deutlich das für Martensit typische Streifenmuster zu erkennen. Beim Entlasten bildet sich dieses Muster zurück, es bleibt jedoch auch hier eine signifikante Dehnung bestehen. Kato et al. erklären diese irreversible Dehnung 
damit, das sich während des Zugversuches Versetzungen durch den Austenit bewegen. Die damit verbundene Plastizität überlagert das superelastische Verhalten des spannungsinduzierten Martensits.

Die Reorientierung von Martensitvarianten der Splats kann in den bei tieferen Temperaturen für kleine Dehnungen aufgenommenen Spannungs-Dehnungskurven besser beobachtet und verstanden werden. Da das Material bereits in der martensitischen Phase vorliegt, kann die Bildung von spannungsinduziertem Martensit von der Reorientierung der Varianten getrennt werden. Wie in Abschnitt 4.3.4 beschrieben, steigt beim Dehnen des Materials die Spannung zunächst linear an, beim Entlasten sinkt sie etwas stärker wieder ab. Dabei geht nur der elastische Anteil der Dehnung zurück und eine plastische Verformung bleibt bestehen. Beim erneuten Dehnen flacht die Steigung der Spannungs-Dehnungskurve ab, sobald der Endzustand des letzten Dehungszyklus erreicht wird. Es setzen wieder plastische Prozesse ein und nach dem Entlasten bleibt eine größere Dehnung der Probe bestehen. Da bei kleinen Dehnungen nur bei niedrigen Temperaturen (im Martensit) und nicht bei hohen Temperaturen (im Austenit) ein plastischer Anteil zurückbleibt, ist dieses Verhalten auf die Umorientierung von Martensitvarianten zurückzuführen. Bei höheren Temperaturen und größeren Dehnungen kommen andere plastische Effekte wie beispielsweise Versetzungsgleiten, Versetzungsklettern oder Korngrenzengleiten oder Zwillingsbildung ${ }^{(1)}$ hinzu [27]. Die wichtigsten Spannungsrelaxationsmechanismen in Eisen-Palladium wurden von Edler und Kühnemund anhand von Molekulardynamiksimulationen und Messungen der mechanischen Spannungen bei der Deposition dünner epitaktischer Eisen-Palladiumschichten Schichten untersucht [66]. Daher ist bekannt, dass zumindest bei hohen Temperaturen in ausscheidungsfreiem Material die mechanische Verspannung vorzugsweise durch den Einbau voller Versetzungen abgebaut wird. Der Einbau von 1/6 $\langle 112\rangle$ ShockleyPartialversetzungen, der zur Bildung von Mikrozwillingen in der fcc-Phase führt, ist hingegen eher in der geordneten $L 1_{0}$ Phase zu erwarten [66].

Sowohl für martensitische als auch für austenitische Proben kann aus dem Abfall der Spannung beim Entlasten der Elastizitätsmodul gewonnen werden. Dabei ist zu beachten, dass in die Berechnung der Spannung aus der anliegenden Kraft die Breite und die Dicke der Probe eingehen. Die Dicke ist jedoch nicht über die gesamte Probe homogen und daher mit einem recht großen Fehler von etwa $10 \%$ behaftet. Auch durch eine ungleichmäßige Einspannung der Probe können Fehler auftreten. Wenn die Probe nicht auf ihrer gesamten Breite gleichmäßg belastet wird, sondern auf einer Seite etwas stärker gezogen wird, muss die gesamte Kraft von dieser Seite der Probe kompensiert werden. Die tatsächlich gedehnte Fläche der Probe ist dann deutlich kleiner als der gemessene Probenquerschnitt. Damit ist auch die tatsächlich auf das Material wirkende Spannung sehr viel größer als angenommen. Entsprechend wird dann der Elastizitätsmodul der Probe unterschätzt. Die Tatsache, dass sich die Probe im martensitischen Zustand beim Entlasten nach großen Dehnungen verdrillt (siehe Abschnitt 4.3.4), spricht dafür, dass sie nicht vollkommen gerade eingespannt war. Die damit einhergehende ungleichmäßige Belastung führte dann auch zum Einreißen der Probe. Der für tiefe Temperaturen gefundene Elastizitätsmodul ist also etwas zu niedrig. Grundsätzlich sind die Größen der in diesem Experiment gefundenen Werte von 13 GPa bzw.

\footnotetext{
${ }^{(1)}$ Auch in der fcc-Phase können Zwillinge entstehen. Diese sind nicht mit den Varianten des Martensit zu verwechseln.
} 
25 GPa für den Elastizitätsmodul durchaus realistisch. Sie sind fast identisch mit den von Kato et al. gefundenen Werten [50]. Des Weiteren liefern Messungen von Edler et al. mit einem Nanoindenter für freistehende einkristalline Schichten ebenfalls Werte der Größenordnung $15 \mathrm{GPa}$ [21].

Besonders interessant ist der Vergleich der Ergebnisse aus den Zugversuchen in dieser Arbeit mit Messungen von Bechtold et al. an einkristallinen $\mathrm{Fe}_{70} \mathrm{Pd}_{30}$-Schichten in der bct-Phase [88], die mit derselben Anlage durchgeführt wurden, an der auch die hier gezeigten Kurven gemessen wurden. Die Form der Spannungs-Dehnungskurven ist fast identisch zu den in dieser Arbeit gemessenen Kurven. Aus dem Abfall der Spannung beim Entlasten wird ebenfalls ein Elastizitätsmodul von $15 \mathrm{GPa}$ gewonnen. Lediglich die plastische Dehnung ist mit insgesamt 0,4\% etwas größer. Dies ist jedoch nicht verwunderlich, da in einkristallinen Schichten die Bewegung von Zwillingsgrenzen weniger durch Defekte behindert wird, so dass bei gleicher Spannung ein höheres Maßs an Reorientierung erreicht werden kann. Das elastische Verhalten einkristalliner Filme und polykristalliner Folien ist also fast identisch, während sich ihr plastisches Verhalten geringfügig unterscheidet.

Bemerkenswert ist dabei, dass entgegen der theoretischen Vorhersage gemäß Abbildung 2.4(a) weder bei hohen noch bei niedrigen Temperaturen ein Martensitplateau auftritt, obwohl die Röntgendiffraktogramme das Auftreten von Martensit belegen. Dies ist zum Teil damit zu erklären, dass für die einzelnen Körner je nach Orientierung der Energiegewinn durch Ausrichten der Martensitvarianten unterschiedlich groß ist. Je kleiner der Winkel zwischen der kurzen Achse und der Dehnungsrichtung ist, desto weiter kann das Korn durch Umorientierung der Spannung nachgeben, und umso größer ist der Energiegewinn. Entsprechend ist eine geringere Spannung notwendig, um die Reorientierung herbeizuführen. Daher gibt es in polykristallinem Material nicht notwendigerweise eine feste Spannung $\sigma_{r}$, bei der die Reorientierung stattfindet, sondern einen ausgedehnten Spannungsbereich, in dem die Martensitvarianten sukzessive ausgerichtet werden.

Allerdings unterliegt die plastische Verformung polykristallinen Materials durch Reorientierung der Martensitvarianten ähnlichen Beschränkungen wie die Verformung durch Abgleiten. Die Verformung durch Reorientierung kann, analog zum Abgleiten, innerhalb eines Korns nur in bestimmte kristallographische Richtungen erfolgen. Da sich ein einzelnes Korn nicht unabhängig von seiner Umgebung verformen kann, muss die Spannung hinreichend groß sein um (analog zur Aktivierung der Schmidschen Gleitsysteme) mehrere Körner unterschiedlicher Ausrichtung zu reorientieren [27]. Zudem ist von anderen Materialsystemen wie z. B. Nickel-Titan bekannt, dass auch in polykristallinem Material ein Martensitplateau auftritt [89]. Das Vorhandensein verschiedener Orientierungen allein verhindert also nicht das Aufreten des Martensitplateaus.

Das Ausbleiben des Martensitplateaus kann auch damit erklärt werden, dass die Struktur nicht entzwillingt, d.h. die Martensitvarianten werden nicht vollständig in Dehnungsrichtung ausgerichtet. Ein solches Verhalten wird durch das Fehlen des fct(200)und (002)-Peaks in den Röntgendiffraktogrammen in Abbildung 4.15 bestätigt. Der Grund hierfür ist in der defektreichen Mikrostruktur der Proben zu finden. Korngrenzen, Versetzungen, mikroskopische Ausscheidungen und andere Defekte behindern die Bewegung der Zwillingsgrenzen durch das Material [66], so dass zur Ausrichtung der Martensitvarianten eine hohe Energiebarriere überwunden werden muss. Zudem kann je nach Art der Defekte und deren lokaler Dichte die zur Ausrichtung nötige Spannung 
der verschiedenen Zwillingsdomänen sehr unterschiedlich sein. Insbesondere wenn die lokalen Gitterverzerungen aufgrund der defektreichen Mikrostruktur eine bestimmte Ausrichtung der Zwillingsdomänen begünstigen, kann die zur Reorientierung nötige Spannung sehr hoch sein. Das Phänomen, dass sich der Martensit durch eine fein verzwillingte Mikrostruktur der umgebenden Matrix anpasst, wurde unter anderem von Khachaturyan et al. beschrieben [90, 91]. Eine solche Struktur wird in der englischen Literatur als adaptive Martensite bezeichnet.

Das Spannungs-Dehnungsverhalten austenitischer Proben ist also durch das Auftreten von stark verzwillingtem spannungsinduziertem Martensit geprägt. Da bei größeren Dehnungen plastische Prozesse auftreten und sich die Martensitvarianten wegen der hohen Defektdichte nicht ausrichten, tritt das charakteristische Martensitplateau nicht auf. Mit einem Elastizitätsmodul von $25 \mathrm{GPa}$ ist das Material erwartungsgemäß sehr weich. Im martensitischen Zustand ist es mit einem Elastizitätsmodul von $13 \mathrm{GPa}$, wie er auch in einkristallinen Filmen beobachtet wird, noch weicher, und es kommt teilweise zu einer Reorientierung der Martensitvarianten.

\subsubsection{Magnetfeldinduzierte Dehnung}

Da die defektreiche Mikrostruktur die Zwillingsbewegung sogar in geglühten Proben offensichtlich stark einschränkt, ist mit dem Aufreten magnetfeldinduzierter Reorientierung nicht zu rechnen. Dennoch zeigt die Messung in Abbildung 4.32 eine deutliche Längenänderung der Probe im Magnetfeld. Konstruktionsbedingt waren Dehnungsmessungen im Magnetfeld nur bei Zimmertemperatur möglich. In diesem Bereich sind Proben der Zusammensetzung $\mathrm{Fe}_{71} \mathrm{Pd}_{29}$ zwar martensitisch, Proben der Zusammensetzung $\mathrm{Fe}_{70} \mathrm{Pd}_{30}$ sind jedoch austenitisch. Da beide Proben eine Längenänderung zeigen, obwohl im Austenit keine magnetfeldinduzierte Reorientierung auftreten kann und die Dehnung beim Abschalten des Magnetfeldes verschwindet, handelt es sich hier um konventionelle Magnetostriktion. Eine Extrapolation der Längenänderung von 0,05\% bei $0,5 \mathrm{~T}$ zum Sättigungsfeld bei ca. 1,2 T ergibt eine Sättigungsmagetostriktion von $1,4 \cdot 10^{-3}$. Dieser Wert liegt deutlich höher als die von Matsui et al. ermittelte Magnetostriktionskonstante von $\lambda_{s}=5 \cdot 10^{-5}$ [1]. Wada et al. fanden jedoch in Polykristallen mit einem Palladiumgehalt von $30 \%$ eine Längenänderung der Größenordnung $10^{-4}$ [92] und Kubota et al. beobachteten in schmelzgesponnenen Bändern der Zusammensetzung $\mathrm{Fe}_{70,4} \mathrm{Pd}_{29,6}$ eine magnetfeldinduzierte Längenänderung von 0,07\% in einem Feld von 0,7 T [93]. Das entspricht genau der Längenänderung, die im vorliegenden Experiment beobachtet wurde. In unbehandelten Splats ist also die magnetfeldinduzierte Reorientierung unterdrückt, es tritt jedoch konventionelle Magnetostriktion auf, deren Ausmaß den Beobachtungen anderer Arbeiten entspricht.

\subsubsection{Aufweichen des Kristallgitters beim Phasenübergang}

Wie schon in Kapitel 2 erwähnt, basieren die Änderungen der Kristallstruktur und die damit einhergehenden Veränderungen von Magnetisierung und Widerstand auf dem Aufweichen des Kristallgitters während des martensitischen Phasenübergangs. Dieses Aufweichen lässt sich anhand der temperaturabhängigen Änderung des Elastizitätsmoduls beim Vibrating-Reed-Experiment in Abbildung 4.27 und 4.28 gut beobachten. 
Zunächst fällt dabei auf, dass in den Proben mit einem Palladiumgehalt von $30 \%$ ein deutlich stärkeres Aufweichen zu beobachten ist als in Proben mit $29 \%$ Palladium. Diese Differenz lässt sich durch die Nähe der $\mathrm{Fe}_{71} \mathrm{Pd}_{29}$-Probe zum Rand des Stabilitätsbereichs der transformierenden Phasen erklären. Da Material dieser Zusammensetzung im Grundzustand die bcc-Struktur annimmt und nur wegen fehlender Relaxationsmöglichkeit in der fct-Phase verbleibt, ist davon auszugehen, dass lokal kleine Bereiche in der bcc-Phase vorliegen. Zum Teil sind diese sogar so groß, dass sie in den Röntgendiffraktogrammen sichtbar werden. Da die bcc-Struktur aus der fct-Struktur lediglich durch eine weitere Verzerrung entlang des Bainpfades entsteht, ist davon auszugehen, dass diese Bereiche in der bcc-Struktur die elastischen Schwingungen nicht wesentlich stärker behindern als Keime des in die fct-Struktur transformierten Materials, sie durchlaufen jedoch keinen weiteren Phasenübergang und tragen somit nicht zum Aufweichen des Gitters bei.

Des Weiteren ist bemerkenswert, dass in den Proben der Zusammensetzung $\mathrm{Fe}_{70} \mathrm{Pd}_{30}$ das Kristallgitter schon bei Temperaturen deutlich oberhalb der Phasenübergangs aufweicht, lange bevor in den Röntgendiffraktogrammen Reflexe der fct-Phase auftauchen. Das Aufweichen des Gitters kann damit als Vorbote für den Phasenübergang betrachtet werden. Aus der Literatur sind verschiedene dieser Precursor-Phänomene bekannt. So beobachten Foos et al. bei Temperaturen oberhalb des Phasenübergangs im Elektronenmikroskop ein charakteristisches Beugungsmuster, das sie als TweedPattern bezeichnen [94]. Oshima et al. assoziieren diese Beugungsmuster mit lokalen Verzerrungsfeldern, die von mikroskopischen Martensitscheibchen verursacht werden [95]. Solche Martensitscheibchen können nach Felten et al. aufgrund lokaler Nahordnung schon bei Temperaturen deutlich oberhalb des Phasenübergangs entstehen [96]. Da der Martensit aus dem Austenit durch eine tetragonale Verzerrung entsteht, muss hier also eine (zumindest lokale) elastische Instabilität vorliegen, wie sie beispielsweise durch das Aufweichen der $\mathrm{TA}_{1}$ Phononenmode gegeben ist [86]. Nach Finlayson entwickelt sich diese Soft Mode, die zu einem Aufweichen der elastischen Konstanten führt, schon bei Temperaturen deutlich oberhalb des Phasenübergangs [97]. Das Aufweichen der elastischen Konstanten wurde für Einkristalle eingehend untersucht [12] und für schmelzgesponnene Bänder bestätigt [98].

In Proben der Zusammensetzung $\mathrm{Fe}_{71} \mathrm{Pd}_{29}$ hingegen beginnt das Gitter erst mit Einsetzen des Phasenüberganges aufzuweichen. Hier fehlt also offenbar jene Instabilität, die nach einer Hypothese von Owen den martensitischen Phasenübergang erst ermöglicht [99]. Auch diese Besonderheit kann damit erklärt werden, dass das Material bei dieser Zusammensetzung eigentlich in der bcc-Phase vorliegen sollte und zum Teil auch vorliegt. Zum einen überlagert der Anstieg des Elastizitätsmoduls der nichttransformierenden bcc-Phase den Abfall des Elastizitätsmoduls der transformierenden Anteile der Probe. Zum anderen liegen diese Proben, wie oben diskutiert, nur wegen der ungünstigen Mikrostruktur in der fct-Phase vor. Wie die Berechnungen von Opahle und Gruner zeigen, können daher die potenzielle Energielandschaft und damit die elastischen Eigenschaften ganz anders sein und ein anderes temperaturabhängiges Verhalten aufweisen als in den Proben der Zusammensetzung $\mathrm{Fe}_{70} \mathrm{Pd}_{30}$.

Das unterschiedlich starke Aufweichen des Kristallgitters vor dem Phasenübergang spiegelt sich auch in den bei Zimmertemperatur mit dem Vibrating-Reed-Experiment bestimmten Elastizitätsmoduli von $72 \mathrm{GPa}$ für die $\mathrm{Fe}_{70} \mathrm{Pd}_{30}$-Probe und $100 \mathrm{GPa}$ für 
die $\mathrm{Fe}_{71} \mathrm{Pd}_{29}$-Probe wider. Es ist anzunehmen, dass sich bei hinreichend hohen Temperaturen beide Proben vollständig in der fcc-Phase befinden und dann auch einen ähnlichen Elastizitätsmodul aufweisen. In der Probe mit $30 \%$ Palladium beginnt das Gitter beim Abkühlen ab einer bestimmten Temperatur deutlich oberhalb des Phasenübergangs aufzuweichen, so dass der Elastizitätsmodul sinkt. In der Probe mit $29 \%$ Palladium hingegen setzt dieses Aufweichen erst bei sehr viel niedrigeren Temperaturen mit Beginn des Phasenüberganges ein und ist weniger stark ausgeprägt. Dadurch sinkt auch der Elastizitätsmodul weniger stark ab. Da bei diesem Palladiumgehalt der Phasenübergang oberhalb von Zimmertemperatur endet, steigt der Elastizitätsmodul sogar wieder ein wenig an, so dass ein wesentlich größerer Wert erreicht wird (siehe Abbildung 4.27).

Kurz gesagt ist also in Proben mit einem Palladiumgehalt von $30 \%$ ein deutliches Aufweichen des Kristallgitters als Vorbote des martensitischen Phasenübergangs zu beobachten. Wegen der Tendenz zur Bildung der bcc-Phase ist das Aufweichen in Proben mit 29 \% Palladium deutlich weniger ausgeprägt, so dass ein größerer Elastizitätsmodul beobachtet wird. Da diese Proben trotzdem transformieren, scheint das Aufweichen des Kristallgitters keine notwendige Voraussetzung für das Auftreten des martensitischen Phasenüberganges zu sein.

\subsubsection{Vergleich der Elastizitätsmoduli}

Beim Vergleich der Werte für den Elastizitätsmodul, die mit dem Vibrating-ReedExperiment und mit dem Zugversuch für Proben der Zusammensetzung $\mathrm{Fe}_{70} \mathrm{Pd}_{30}$ bestimmt wurden, fällt auf, dass der im Vibrating-Reed-Experiment bestimmte Modul etwa dreimal so groß ist wie der aus dem Zugversuch gewonnene Wert. Diese Diskrepanz kann durch systematische Fehler der beiden Aufbauten sowie die unterschiedlichen Zeitskalen und die verschiedenen Geometrien der experimentellen Anordnung und der untersuchten Dehnungen erklärt werden.

Wie bereits erwähnt, kann bei dem Zugversuch der Elastizitätsmodul systematisch unterschätzt werden, wenn die Probe nicht ganz gleichmäßig eingespannt wird oder sich bei großen Spannungen das Gestänge der Zugprüfanlage mitdehnt. Bei dem VibratingReed-Experiment hingegen werden die Elastizitätsmoduli tendenziell eher überschätzt. Wenn die Probe nicht vollkommen eben eingespannt wird, sondern leicht quer zur Biegerichtung gewölbt ist, führt dies zu einer dramatischen Versteifung in Biegerichtung. Entsprechend steigen die Resonanzfrequenz und der daraus berechnete Elastizitätsmodul.

Doch auch bei perfektem Einbau der Proben müssen die aus den beiden verschiedenen Experimenten gewonnenen Elastizitätsmoduli nicht unbedingt übereinstimmen, da im Vibrating-Reed-Experiment unbehandelte Proben untersucht wurden, während bei den Zugversuchen geglühte Proben zum Einsatz kamen. Die Veränderung der Mikrostruktur und der Abbau von Defekten beim Glühen können dazu führen, dass das Material erheblich weicher wird, so dass in den Zugversuchen an geglühten Proben ein niedrigerer Elastizitätsmodul gemessen wird.

Außerdem ist zu berücksichtigen, dass sich die Zeitskalen der beiden Experimente deutlich unterscheiden. So spielt bei einer zyklischen Belastung, wie sie an dem schwingen- 
den Biegebalken auftritt, auch das anelastische Verhalten des Materials eine Rolle [27]. Bei hinreichend hohen Frequenzen bzw. großen Relaxationszeiten wird hier der unrelaxierte Modul $E_{U}$ gemessen, während im quasistatischen Zugversuch eher der relaxierte Modul $E_{R}$ zugänglich ist. Für die meisten Metalle gilt $E_{U}>E_{R}$ [27], im VibratingReed-Experiment ist also ein etwas höherer Modul zu erwarten als im Zugversuch. Auch wenn die verschiedenen Zeitskalen nicht die gesamte Differenz zwischen den Elastizitätsmoduli erklären, stimmt die erwartete Tendenz mit der experimentellen Beobachtung überein.

Auch die Geometrie der experimentellen Anordnung kann den gemessenen Wert des Elastizitätsmoduls beeinflussen. Eine solche Diskrepanz zwischen Elastizitätsmoduli, die mit verschiedenen Methoden mit unterschiedlichen Geometrien an der gleichen Probe bestimmt wurden, beobachten beispielsweise Bechtold et al. [88]. Nanoindentationsmessungen an epitaktisch gewachsenen $\mathrm{Fe}_{70} \mathrm{Pd}_{30}$-Filmen in der bct-Struktur ergaben einen Elastizitätsmodul von $140 \mathrm{GPa}$, während Zugversuche an einem freistehenden einkristallinen Film nur $15 \mathrm{GPa}$ lieferten. Bechtold et al. versuchen, diesen Unterschied mit der unterschiedlichen Geometrie der mechanischen Belastung des Materials und der damit einhergehenden Deformation zu erklären. Während im Zugversuch eine uniaxiale Spannung entlang der [110] $]_{c t}$-Richtung angelegt wird, wirkt an der Spitze des Nanoindenters eine multiaxiale Kraft. Die unterschiedlichen Werte der Elastizitätsmoduli seien auf die große elastische Anisotropie des Materials zurückzuführen, welches bei uniaxialer Dehnung weicher sei als bei einer Verformung in verschiedene Richtungen. Des Weiteren komme es bei der Nanoindentation zu signifikanter plastischer Verformung, die das elastische Verhalten des umgebenden Materials beeinflusse. Die Argumentation von Bechtold et al. lässt sich jedoch nicht direkt auf die in dieser Arbeit untersuchten Proben und Methoden übertragen. Zum einen sind die hier untersuchten Proben polykristallin, weisen also keine signifikante elastische Anisotropie auf, und die Verformung im Vibrating-Reed-Experiment ist nicht multiaxial. Zum anderen ist gerade im Vibrating-Reed-Experiment keine plastische Verformung zu erwarten.

Dennoch können die verschiedenen Arten der mechanischen Belastung zu unterschiedlichen Elastizitätsmoduli führen. So sind die im Vibrating-Reed-Experiment erreichten Dehnungen sehr viel kleiner als die Auflösungsgrenze im Zugversuch. Aus dem Verlauf der potentiellen Energielandschaft in Abbildung 2.11 ist klar zu erkennen, dass das interatomare Potential deutlich von dem idealen quadratischen Verhalten abweicht. Daher ist die rückstellende Kraft einer Dehnung, also auch der Elastizitätsmodul, nicht dehnungsunabhängig. Gerade für große Dehnungen ist die Energielandschaft deutlich flacher als die Energiekurve für die reinen Elemente Eisen und Palladium, die in Abbildung 2.11 eingezeichnet ist, und hat insbesondere eine sehr viel geringere Krümmung. Da in einem näherungsweise isotropen polykristallinen Material der Elastizitätsmodul ähnlich wie die Kompressibilität eng mit der Krümmung des interatomaren Potentials verknüpft ist [100], wird entsprechend für große Dehnungen ein geringerer Elastizitätsmodul gefunden.

Des Weiteren ist zu berücksichtigen, dass im Vorfeld des martensitischen Phasenübergangs nur einzelne Phononenmoden aufweichen. Aufgrund der unterschiedlichen Geometrien, die in den beiden Experimenten realisiert werden, ist es möglich, dass sich diese Anisotropie unterschiedlich stark auswirkt. Während im Zugexperiment eine reine Dehnung auftritt, kommt es beim Biegebalken gleichzeitig zu einer Dehnung auf 
der einen und einer Stauchung auf der anderen Seite des Balkens. Dadurch werden die Atome stärker gegeneinander geschert als in der reinen Dehnung (die allerdings bei geeigneter Wahl des Bezugssystems auch als Überlagerung einer Scherung und einer Kompression betrachtet werden kann [101]). Da die $\mathrm{TA}_{1}$-Phononenmode eine Scherinstabilität bewirkt [102], kann es so zu geometriebedingten Unterschieden im elastischen Verhalten kommen.

Der Vergleich der Elastizitätsmoduli, die aus dem Vibrating-Reed-Experiment und den Spannungs-Dehnungskurven gewonnen wurden, liefert also mehrere Effekte, die zu dem beobachteten Unterschied führen können. Neben Ungenauigkeiten beim Einbau der Proben können die unterschiedlichen Zeitskalen und Dehnungsamplituden der beiden Experimente zu unterschiedlichen Werten der Elastizitätsmoduli führen.

\subsubsection{Dämpfung}

Das Verhalten der Dämpfung ist bei einem Palladiumgehalt von $29 \%$ oder $30 \%$ sehr ähnlich und scheint nicht direkt mit der eben diskutierten Änderung des Elastizitätsmoduls verknüpft zu sein. Wie in Abbildung 4.27 und 4.28 zu sehen, ist die Dämpfung bei hohen Temperaturen weitestgehend konstant und beginnt während des Phasenüberganges erwartungsgemäß stark anzusteigen. Dieser Anstieg setzt sich bis zu tiefen Temperaturen fort. Im Prinzip würde man erwarten, dass die Dämpfung während des Phasenüberganges ein Maximum aufweist und anschließend wieder abfällt [103]. Nakajima et al. haben Dämpfungsmessungen an schmelzgesponnenen Bändern mit einem Palladiumgehalt von 29,6\% durchgeführt und beobachten mehrere Maxima der Dämpfung, die sie dem martensitischen Phasenübergang zuschreiben [98]. Allerdings sind bei den Temperaturen, bei denen diese Maxima auftreten, keine Änderungen des Elastizitätsmoduls oder andere Änderungen der Probeneigenschafte zu beobachten, die auf den martensitischen Phasenübergang hindeuten. Zudem ist die Dämpfung, die Nakajima et al. bei Temperaturen oberhalb von Zimmertemperatur beobachten, mit etwa $Q^{-1}=3 \cdot 10^{-3}$ um ein Vielfaches größer als die Dämpfung, die in dieser Arbeit an Splats beobachtet wurde. Geht man davon aus, dass schmelzgesponnene Bänder und Splats, die beide mit ähnlichen Raten aus der flüssigen Phase abgeschreckt wurden, ähnliche intrinsische Eigenschaften haben sollten, liegt die Vermutung nahe, dass die von Nakajima et al. beobachtete Dämpfung nicht alleine die intrinsische Dämpfung der Probe wiedergibt, sondern dass hier auch apparative Störeffekte, beispielsweise von der Einspannung, eine Rolle spielen.

Um einen rein apparativen Effekt der Dämpfung auszuschließen, wurden daher Kontrollmessungen mit einem $150 \mu \mathrm{m}$ dicken einkristallinen Siliziumplättchen durchgeführt. Silizium ist für seine geringe intrinsische Dämpfung bekannt [74], so dass davon auszugehen ist, dass die bei dieser Messung auftretende Dämpfung alleine auf die Dämpfung der Einspannung zurückzuführen ist. Der temperaturabhängige Verlauf der Dämpfung des Siliziumplättchens ist in Abbildung 5.4 gemeinsam mit einer Dämpfungsmessung an einem Splat der Zusammensetzung $\mathrm{Fe}_{70} \mathrm{Pd}_{30}$ gezeigt. Es fällt sofort auf, dass die Dämpfung des Siliziumplättchens, bzw. die apparative Dämpfung, etwa eine Größenordnung kleiner ist als die Dämpfung des Splats. Der typische, bei allen Splats beobachtete Anstieg der Dämpfung bei tiefen Temperaturen tritt bei der Messung mit dem Siliziumplättchen nicht auf. Stattdessen ist sogar ein leichter Abfall der 


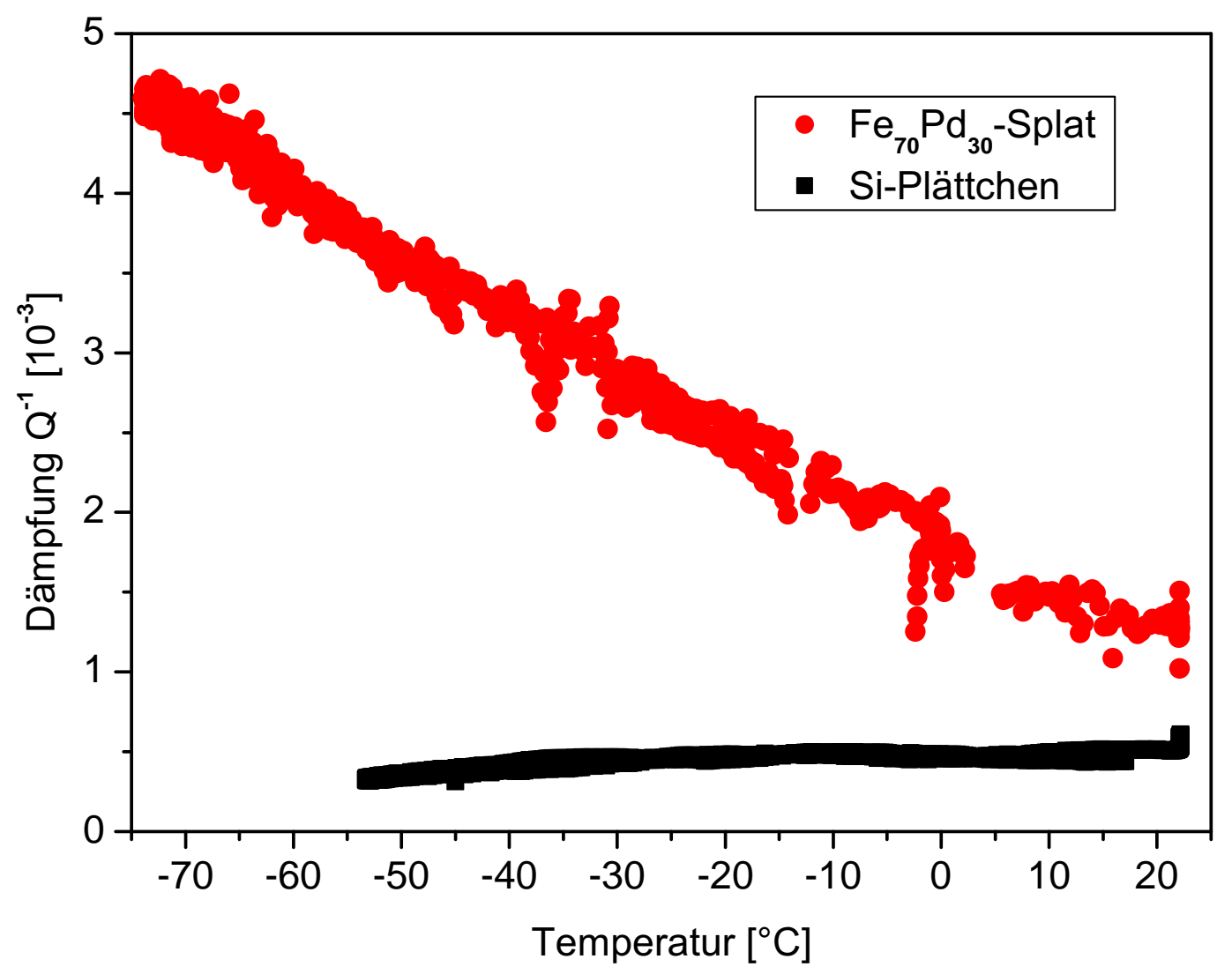

Abbildung 5.4: Die Dämpfung des Splats steigt auch bei tiefen Temperaturen weiter an. Die Dämpfung des Siliziumplättchens hingegen fällt leicht ab und ist insgesamt sehr viel kleiner.

Dämpfung mit fallender Temperatur zu beobachten. Es ist also davon auszugehen, dass sich bei dieser Messung die Einspannung nicht gelockert hat.

Es bleibt jedoch noch zu erklären, warum in den Messungen an Splats in Abbildung 4.27 und 4.28 nach Beendigung des Phasenüberganges kein Abfall der Dämpfung beobachtet wird. Dafür kann es verschiedene Gründe geben. Auch wenn die hohe Dämpfung nicht allein auf das Verhalten der Einspannung zurückzuführen ist, kann ein Effekt der Einspannung nicht ausgeschlossen werden, da immer das Zusammenspiel von Probe und Einspannung betrachtet werden muss. Es ist möglich, dass sich bei tiefen Temperaturen die Einspannung lockert, wenn sich die thermischen Ausdehnungskoeffizienten von Einspannung und Probe unterscheiden. Der lineare Ausdehnungskoeffizient von Silizium liegt bei $2 \cdot 10^{-6} \mathrm{~K}^{-1}$. Der Ausdehnungskoeffizient von Edelstahl ist mit $10 \cdot 10^{-6} \mathrm{~K}^{-1}$ bis $16 \cdot 10^{-6} \mathrm{~K}^{-1}$ deutlich kleiner, so dass sich die Einspannung beim Abkühlen stärker zusammenzieht als das Siliziumplättchen. Damit wird das Plättchen bei tiefen Temperaturen fester geklemmt. Die thermische Ausdehnung von Eisen-Palladium hängt stark von der Temperatur ab. Bei Zimmertemperatur beträgt sie etwa $10 \cdot 10^{-6} \mathrm{~K}^{-1}$ und nimmt mit fallender Temperatur ab [3, 4]. Damit ist es auch hier unwahrscheinlich, dass sich die Einspannung bei tiefen Temperaturen stark lockert, ein solcher Effekt kann jedoch nicht vollständig ausgeschlossen werden.

Auch wenn die thermische Volumenänderung von Eisen-Palladium nahe Zimmertemperatur ähnlich ist wie bei Edelstahl, besteht trotzdem die Möglichkeit, dass sich die 
Einspannung lockert. Innerhalb der Einspannung übt die Klemmung eine uniaxiale Kompression auf die Probe aus, die schon bei Temperaturen etwas oberhalb des Phasenüberganges zur Bildung von Martensit führen kann. Die Martensitvarianten können sich so ausrichten, dass die Kompression zum Teil kompensiert wird. Da die Einspannung bei Zimmertemperatur festgezogen wurde, ist davon auszugehen, dass sich der spannungsinduzierte Martensit direkt beim Einbau der Probe bildet, so dass dieser Effekt zunächst nicht zum Lockern der Einspannung führen kann. Die Röntgendiffraktogramme und die temperaturabhängige Änderung des Gitterparameters in Abbildung 4.17 und 4.18(a) zeigen jedoch, dass sich die tetragonale Verzerrung auch nach dem Phasenübergang noch erhöht. Das heißst, dass sich auch die Länge der kurzen $c$-Achse weiter reduziert, so dass die Probe dem Druck der Einspannung weiter nachgeben kann. Damit sinkt mit fallender Temperatur die Kraft, die von der Einspannung auf die Probe ausgeübt wird.

Dass die Dämpfung beim Einsetzen des Phasenüberganges ansteigt, jedoch nicht wieder abfällt, könnte auch daran liegen, dass der Phasenübergang bei Beendigung der Messung in Abbildung 4.28 noch nicht vollständig abgeschlossen ist. Aus den Röntgendiffraktogrammen und der Änderung des Gitterparameters in Abbildung 4.17(a) und 4.18(a) ist zu erkennen, dass der Phasenübergang in unbehandelten Proben, wie sie für das Vibrating-Reed-Experiment eingesetzt wurden, über einen sehr weiten Temperaturbereich ausgeschmiert und bei $-25^{\circ} \mathrm{C}$ noch nicht vollständig abgeschlossen ist. Dies reicht jedoch als Erklärung für das Fehlen des Abfalls nicht aus, da auch in Dämpfungsmessungen bis zu einer Temperatur von $-70^{\circ} \mathrm{C}$ (siehe Abbildung 5.4) weder ein Abfall der Dämpfung noch ein Abflachen der Steigung beobachtet wurde.

Alternativ kann der Anstieg der Dämpfung nach dem Phasenübergang mit dem Einsetzen von Zwillingsbewegung in der martensitischen Phase erklärt werden. Auch wenn die bei einem Vibrating-Reed-Experiment auftretenden Dehnungen sehr klein sind, können die dabei entstehenden Spannungen lokal zur Bewegung von Zwillingsgrenzen führen. Aus der Literatur ist bekannt, dass das Einsetzen der Zwillingsbewegung zu einem Anstieg der Dämpfung führt [104]. Mit fallender Temperatur steigt der Anteil des Materials in der martensitischen Phase. Damit stehen mehr Zwillingsgrenzen zur Verfügung, die sich durch das Material bewegen können. Außerdem erhöht sich die tetragonale Verzerrung, so dass durch Umorientieren von Martensitvarianten mehr elastische Energie gewonnen werden kann. Damit entsteht eine größere treibende Kraft zur Reorientierung der Varianten, die eine verstärkte Bewegung der Zwillingsgrenzen zur Folge hat. Da die Bewegung von Zwillingsgrenzen ein dissipativer Prozess ist, steigt dabei die Dämpfung.

Die obige Diskussion zeigt, dass für den Anstieg der Dämpfung nach dem Phasenübergang mehrere Erklärungen in Betracht kommen. Ein rein apparativer Effekt, der durch Lockern der Einspannung beim Kühlen verursacht wird, ist unwahrscheinlich. Es ist eher davon auszugehen, dass der Anstieg mit dem Phasenübergang verknüpft ist. So könnte sich die Einspannung wegen der lokalen Bildung von spannungsinduziertem Martensit lockern. Alternativ kann der Anstieg mit der Überlagerung eines breiten Dämpfungspeaks, der von der ausgeschmierten Transformation verursacht wird und dem Einsetzen der Zwillingsbewegung im Martensit erklärt werden. 


\subsection{Der martensitische Phasenübergang}

Neben den eben diskutierten mechanischen Eigenschaften ändern sich während des martensitischen Phasenübergangs auch die Kristallstruktur, die Magnetisierung und der elektrische Widerstand der Proben. Diese Änderungen sollen nun genauer diskutiert werden. In Abbildung 5.5 ist zu erkennen, dass sich alle drei Größen bei der gleichen Temperatur ändern und somit als Indikatoren für den Phasenübergang herangezogen werden können. Darüber hinaus deutet die überraschende Ähnlichkeit des Verlaufs der Änderung des Gitterparameters und der Magnetisierung in einem kleinem Magnetfeld auf eine enge Verknüpfung der beiden Größen hin. In den beiden folgenden Abschnitten soll daher erörtert werden, wie sich Veränderungen der Kristallstruktur auf den Widerstand und die Magnetisierung auswirken, um die Ursachen für diese kollektive Änderung herauszuarbeiten. Anschließend wird in Abschnitt 5.4.3 analysiert, auf welche Art eine Glühbehandlung und die damit einhergehende Veränderung der Mikrostruktur den martensitischen Phasenübergang beeinflussen.

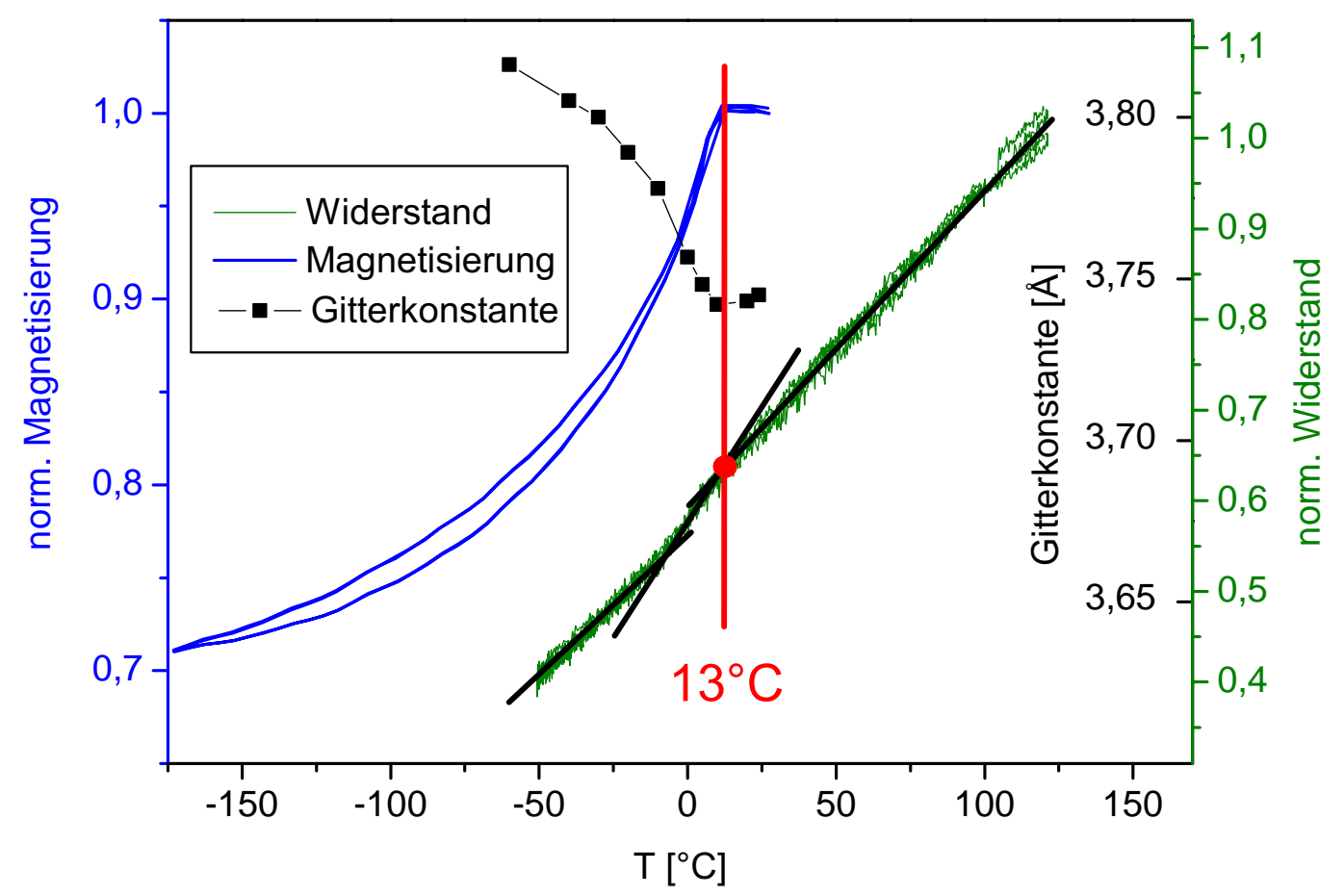

Abbildung 5.5: Der martensitische Phasenübergang bewirkt eine kollektive Änderung von Kristallstruktur, Magnetisierung und elektrischem Widerstand.

\subsection{1 Änderung der Magnetisierung}

Generell ist in Legierungen der Übergangsmetalle eine deutliche Wechselwirkung zwischen Kristallstruktur, Bandstruktur und Magnetismus zu erwarten. Eine Veränderung der Bandstruktur aufgrund der Verzerrung der Einheitszelle würde allerdings in erster Linie zu einer Änderung des magnetischen Moments je Einheitszelle führen. Eine solche Änderung wäre in der Sättigungsmagnetisierung deutlich zu erkennen. Aus der 
Literatur ist jedoch bekannt, dass sich die Sättigungsmagnetisierung beim Übergang von Austenit zu Martensit nur unwesentlich ändert [6]. Zudem ist sie im Martensit geringfügig größer als im Austenit [6]. Die Magnetisierungsmessungen in dieser Arbeit zeigen jedoch genau gegenteiliges Verhalten: Beim Übergang in den Martensit sinkt die Magnetisierung. Der Abfall der Magnetisierung beim Kühlen kann also nicht einfach einer Änderung der Sättigungsmagnetisierung zu geschrieben werden. Zudem ist zu berücksichtigen, dass die Magnetisierungskurven in einem kleinen Feld von nur $30 \mathrm{mT}$ aufgenommen wurden, das weit vom Sättigungsfeld von etwa 1,2 T entfernt ist.

Bei solch kleinen Feldern kann die Ausrichtung der magnetischen Domänen durch kleine Störungen stark beeinflusst werden. Das Material ist jedoch sehr weichmagnetisch, so dass im Austenit ein großer Teil der Domänen entlang der Feldrichtung orientiert ist. Damit ist die makroskopische Magnetisierung der Probe recht hoch. Mit der tetragonalen Verzerrung der Einheitszelle beim martensitischen Phasenübergang entwickelt sich jedoch eine magnetische Anisotropie entlang der langen Achsen [105]. Mit zunehmender Anisotropie wird eine Ausrichtung der magnetischen Momente entlang der leichten Richtung, also der langen Achsen, immer günstiger. Sofern das angelegte Feld hinreichend klein ist, rotieren die magnetischen Momente aus der Richtung des angelegten Feldes in Richtung der langen Achsen. Da in polykristallinem Material die langen Achsen in alle Richtungen verteilt sind, kommt es so zu einer Demagnetisierung der Probe. Der beobachtete Abfall der Magnetisierung ist also ein Indikator für das Entstehen der großen magnetischen Anisotropie [106], die für das Auftreten des magnetischen Formgedächtniseffektes notwendig ist.

\subsection{2 Änderung des Widerstands}

Der Anstieg der magnetischen Anisotropie und die damit einhergehende Veränderung der Domänenstruktur muss auch als Ursache für die Änderung des elektrischen Widerstandes bei der Transformation in Erwägung gezogen werden. Aus der Literatur ist bekannt, dass der Widerstand ferromagnetischer Materialien von ihrem magnetischen Zustand abhängt und damit durch ihre Anisotropie, Magnetisierung und Domänenstruktur beeinflusst wird [107]. So wurde beispielsweise von Danneau et al. ein Anstieg des Widerstandes in Eisen-Palladium durch Streuung der Leitungselektronen an magnetischen Domänengrenzen nachgewiesen [107]. Es ist denkbar, dass in einem Zustand erhöhter magnetischer Anisotropie die Domänengröße steigt und damit weniger Streuzentren zur Verfügung stehen, so dass der elektrische Widerstand leicht absinkt. Alternativ muss jedoch auch ein direkter Einfluss der Bandstruktur auf den elektrischen Widerstand in Betracht gezogen werden. Aus Berechnungen von Opahle et al. ist bekannt, dass die tetragonale Verzerrung der Einheitszelle bei der Transformation durch eine Jahn-Teller-artige Aufspaltung der $d$-Bänder verursacht wird [53]. Dabei verschieben sich Majoritäts- und Minoritäts-Spin-Zustände gegeneinander und es kommt zu einer verminderten Zustanddichte der Minoritäts-Spins direkt an der Fermi-Kante. Durch die Reduktion der Zustandsdichte an der Fermi-Energie stehen weniger Zustände zur Verfügung, in welche die Leitungselektronen hineinstreuen können. Mit der Streuwahrscheinlichkeit der Leitungselektronen sinkt auch der elektrische Widerstand [108]. 


\subsubsection{Einfluss der Mikrostruktur auf den Phasenübergang}

Der folgende Abschnitt wird zeigen, dass der Phasenübergang stark durch die Mikrostruktur beeinflusst wird. Insbesondere haben Gitterverzerrungen und Korngröße einen deutlichen Einfluss auf die Übergangstemperatur. Theoretisch ist es möglich, durch genaue Analyse der Form der Röntgenreflexe Informationen über Korngröße und Gitterverzerrungen zu gewinnen. Der Einfluss der mittleren Korngröße über die Kohärenzlänge auf die Breite von Röntgenreflexen ist allgemein bekannt und kann mit der Scherrerformel (Gleichung 4.1) abgeschätzt werden. Lokale mikroskopische Verspannungen $\sigma$ führen jedoch ebenfalls zu einer Verbreiterung $w_{\sigma}$ eines Reflexes unter dem Streuwinkel $\theta$, gemäß

$$
\sigma=\frac{E w_{\sigma}}{4 \tan \theta}
$$

mit dem Elastizitätsmodul E [109]. Da die Verteilungen von Verspannung und Korngrößen unterschiedlichen Statistiken folgen, kann die Verbreiterung aufgrund von Verspannungen durch Anpassen einer Gaußfunktion erfolgen, während der Einfluss der Korngröße durch eine Lorentzfunktion repräsentiert wird. In einem lokal verspannten Material mit endlicher Korngröße kann die Form der Röntgenreflexe also durch eine Überlagerung einer Lorentz- und einer Gaußfunktion, einer sogenannten Voigtfunktion, beschrieben werden [110, 111]. Sollen in der Praxis jedoch aus der Form der Reflexe Verspannung und Korngröße gewonnen werden, treten Schwierigkeiten auf. In den meisten Fällen kommt es in Röntgenspektren zu Überlagerungen mehrerer Reflexe, sowohl von den Kristallebenen verschiedener Phasen, als auch von den unterschiedlichen Wellenlängen der $K_{\alpha 1^{-}}$und $\mathrm{K}_{\alpha 2^{2}}$-Strahlung. Es müssen also mehrere Voigtfunktionen mit jeweils fünf freien Parametern angepasst werden. Dabei besteht die Gefahr, dass der Fit-Algorithmus zu einem lokalen statt globalen Minimum des Anpassungsfehlers konvergiert bzw. dass sehr unterschiedliche Parametersätze näherungsweise zum gleichen Anpassungsfehler führen. Damit wird es unmöglich, die Beiträge der Lorentz- und Gaußfunktionen, bzw. der Korngröße und Verspannungen zu unterscheiden. Aus diesem Grund kann die Form der Röntgenpeaks in der folgenden Diskussion nicht genutzt werden, um weitere Informationen über die Korngröße und die lokalen Verspannungen zu gewinnen. Auch mit anderen röntgenographischen Verfahren wie bespielsweise der $\sin ^{2} \theta$-Methode sind die lokalen Verspannungen nicht zugänglich, da diese Methode nur Information über makroskopische Verspannungen liefert. Deshalb können Informationen über die Veränderung der Korngröße während der Glühbehandlung nur aus den REM- und TEM-Bildern in Abschnitt 4.1 gewonnen werden.

Im Folgenden soll diskutiert werden, inwieweit die Transformationstemperatur von binären Proben der Zusammensetzung $\mathrm{Fe}_{70} \mathrm{Pd}_{30}$ durch die Präparation, insbesondere durch die Wahl einer geeigneten Glühbehandlung, beeinflusst werden kann. Da davon auszugehen ist, dass sich beim Glühen nicht die Zusammensetzung der Proben, sondern nur ihre Mikrostruktur verändert, kann deren Einfluss auf das Transformationsverhalten untersucht werden.

Während des Phasenüberganges zeigen Kristallstruktur, Widerstand und Magnetisierung der Proben eine deutliche Veränderung. Für die meisten Proben passen die aus der Änderung der verschiedenen Größen bestimmten Transformationstemperaturen sehr gut zusammen. Lediglich die bei $700{ }^{\circ} \mathrm{C}$ geglühte Probe zeigt ein deutlich 
abweichendes Verhalten. Mit keiner der verwendeten Messmethoden konnte ein klarer Phasenübergang nachgewiesen werden. Die Röntgendiffraktogramme zeigen deutliche Eisenausscheidungen und die EDX-Analyse bestätigt die inhomogene Verteilung des Eisengehalts. Wie in Abschnitt 5.1 erwähnt, ist dies darauf zurückzuführen, dass die Glühtemperatur von $700{ }^{\circ} \mathrm{C}$ dicht unterhalb der Mischbarkeitsgrenze liegt, so dass bei hinreichend großer treibender Kraft zum Entmischen genug Diffusion stattfindet, um makroskopische Ausscheidungen zu bilden. Da in dieser Probe kein deutlicher Phasenübergang nachgewiesen werden konnte, soll sie in der folgenden Diskussion ausgespart werden.

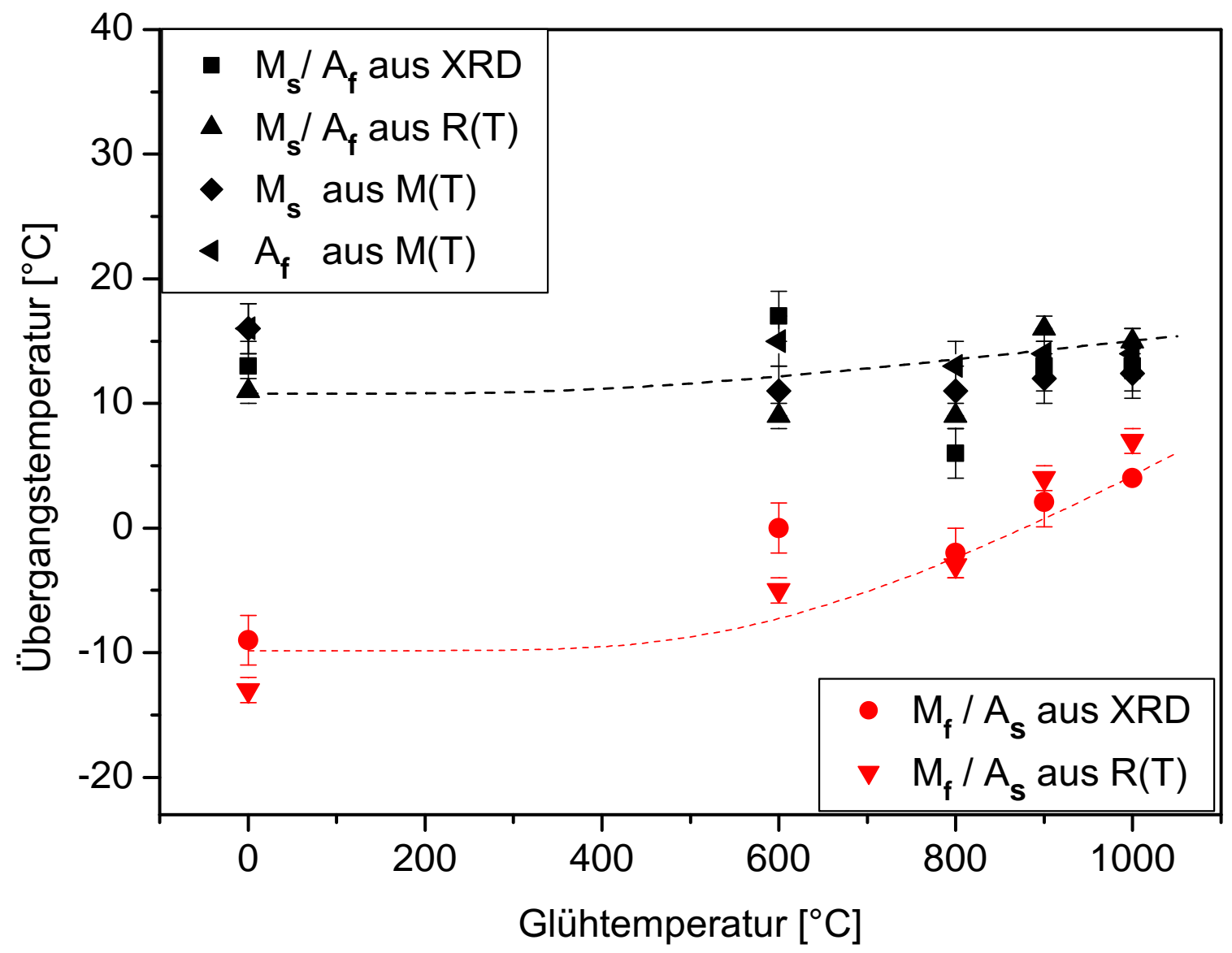

Abbildung 5.6: Mit steigender Glühtemperatur erhöht sich die Übergangstemperatur und der Phasenübergang wird schärfer.

Die Übergangstemperaturen der anderen Proben zeigen einen Anstieg mit steigender Glühtemperatur. In Abbildung 5.6 sind die mit Hilfe der Peakverschiebung in den Röntgendiffraktogrammen und dem Abfall von Widerstand und Magnetisierung bestimmten Werte für die Martensitstart- bzw. Austenitendtemperatur $\mathrm{M}_{s} / \mathrm{A}_{f}$ und die Austenitstart- bzw. Martensitendtempertur $\mathrm{A}_{s} / \mathrm{M}_{f}$ gegen die Glühtemperatur aufgetragen. Es fällt sofort auf, dass die $\mathrm{A}_{s} / \mathrm{M}_{f}$-Kurve deutlich steiler ansteigt, d.h. der Phasenübergang wird mit steigender Glühtemperatur schärfer. Die gestrichelte rote Linie unterstreicht den Arrhenius-artigen Anstieg der Übergangstemperatur mit steigender Glühtemperatur. Um diesen Anstieg zu verstehen, bietet sich eine genauere Betrachtung der Parameter an, die den Phasenübergang beeinflussen. 
Aus der Literatur ist bekannt, dass die Transformationstemperatur im Wesentlichen durch den Eisengehalt [6], die Defektdichte [112] und die mechanische Spannung in der Probe [50] beeinflusst wird. Diese Größen hängen beispielsweise von der Homogenität der Probe, der Abschreckgeschwindigkeit, der Korngröße und -form oder dem Vorhandensein von Ausscheidungen ab, die sich wiederum gegenseitig beeinflussen. Darüber hinaus ist es notwendig, zwischen Faktoren zu unterscheiden, die die Temperatur $T_{0}$ beeinflussen, bei der sich Austenit und Martensit im thermodynamischen Gleichgewicht befinden, und anderen Faktoren, die die Transformationstemperatur über die notwendige Unterkühlung $\Delta T=T_{0}-M_{s}$, die als treibende Kraft für die Transformation wirkt, beeinflussen.

Bei der Probenpräparation wurde sorgfältig darauf geachtet, die Homogenität des Eisengehalts der Splats sicherzustellen. Die Möglichkeit, dass geringfügige Konzentrationsunterschiede bis zu $0,3 \%$ bestehen, konnte jedoch auch mit Hilfe des $\mathrm{Fe}_{70} \mathrm{Pd}_{30^{-}}$ Standards mit dem EDX nicht ausgeschlossen werden, da diese geringen Unterschiede unter der Empfindlichkeitsgrenze des Geräts liegen. Da die thermodynamische Gleichgewichtstemperatur $T_{0}$ mit $\mathrm{d} T_{0} / \mathrm{d} c_{F e} \approx 20^{\circ} \mathrm{C} /$ at.\% stark vom Eisengehalt $c_{F e}$ abhängt [6], können schon geringste Schwankungen des Eisengehalts die Übergangstemperatur unabhängig von Einflüssen der Glühbehandlung um mehrere Grad Celsius verschieben.

Da zudem das System Eisen-Palladium bei tiefen Temperaturen nicht mischbar ist, besteht eine treibende Kraft, die laut Felten et al. auch bei raschem Abschrecken zur Bildung mikroskopischer Gebiete mit erhöhtem Eisengehalt führt, die bei deutlich höheren Temperaturen transformieren als die umgebende Matrix [96]. Zudem bildet sich eine lokale Nahordnung aus, welche die tetragonale Verzerrung begünstigt, so dass diese schon deutlich oberhalb der Transformationstemperatur lokal auftritt [96]. So können mikroskopische Eisenanreicherungen, die mit EDX nicht nachweisbar sind, zu einer Erhöhung der Übergangstemperatur führen. Derartige Fluktuationen des Eisengehalts sind preferentiell in jenen Proben zu erwarten, die unterhalb der Mischbarkeitstemperatur von $760{ }^{\circ} \mathrm{C}$ geglüht wurden. Wie erwähnt, treten in der bei $700{ }^{\circ} \mathrm{C}$ geglühten Probe makroskopische Fluktuationen auf, so dass in dieser Probe der Phasenübergang unterdrückt ist. In der bei $600^{\circ} \mathrm{C}$ geglühten Probe treten zwar auch Eisenausscheidungen auf, mit dem EDX sind jedoch keine Fluktuationen zu erkennen, so dass hier möglicherweise die lokalen Schwankungen vorherrschen und so die leichte Erhöhung der Übergangstemperatur gegenüber der Erwartung erklären.

Die Defektdichte und -verteilung innerhalb der Proben ist ebenfalls nicht vollständig identisch. Schon innerhalb einer Probe bestehen große Unterschiede zwischen dem Inneren und dem Rand der Splats, wie in Abbildung $4.1 \mathrm{zu}$ erkennen ist. Diese entstehen, wenn das ursprünglich runde Tröpfchen beim Abschrecken zwischen den Kupferstempeln flach gedrückt wird. Ist die Defektdichte hoch, so bestehen viele Nukleationszentren für die martensitische Phase [112], und es können sich leichter Keime der Martensitphase bilden. In einem stark verzerrten Gitter sind diese unter Umständen auch schon bei höheren Temperaturen stabil. Damit sollte eine hohe Defektdichte mit hohen Transformationstemperaturen einhergehen. Während des Auslagerns können einige dieser Defekte ausheilen, und zwar umso mehr, je höher die Glühtemperatur ist. Damit müsste die Umwandlungstemperatur mit steigender Glühtemperatur sinken. Dies entspricht jedoch nicht der experimentellen Beobachtung. Andererseits können Defekte die Bewegung der Phasengrenze durch das Material behindern. Insbesondere stellen 
Ausscheidungen, die sich bei Glühtemperaturen unter $800{ }^{\circ} \mathrm{C}$ gebildet haben, ein Hindernis für die Phasengrenze dar, so dass eine größere Unterkühlung notwendig wird und das Material bei niedrigeren Temperaturen transformiert [113].

Da der Anstieg der Martensitendtemperatur sehr viel deutlicher ist als jener der Martensitstarttemperatur und die Hysterese zwischen Heizen und Kühlen so gering ist, dass mit den meisten Methoden kein Unterschied zwischen Martensitstart- und Austenitendtemperatur gefunden wurde, ist davon auszugehen, dass die Martensitphase durch Verschiebung der Phasengrenze und nicht durch Bildung neuer Keime, sogenannter Martensitembryos wächst.

Wie oben erwähnt, kann auch der Spannungszustand der Proben den martensitischen Phasenübergang beeinflussen. So ist das Auftreten spannungsinduzierten Martensits insbesondere von dünnen Schichten wohlbekannt [84]. Da die Splats jedoch nicht mit einem festen Substrat verbunden sind, kann der größte Teil der mechanischen Verspannungen, die bei der Präparation in die Proben eingebracht werden, durch Ausdehnen oder Krümmen abgebaut werden. In rasch abgeschreckten polykristallinen Proben bleibt jedoch immer eine intrinsische Verspannung durch Gitterverzerrungen an Defekten bestehen. Wie bereits in Abschnitt 5.4.3 dargestellt, ist eine Quantifizierung dieser lokalen Verspannungen mit Hilfe der Röntgendiffraktogramme schwierig, da die Breite der Röntgenreflexe nicht nur durch die lokalen Verspannungen, sondern auch durch die Korngröße beeinflusst wird.

Es ist davon auszugehen, dass in der unbehandelten und den bei niedrigeren Temperaturen geglühten Proben größere Verspannungen vorhanden sind als in den bei hohen Temperaturen geglühten Proben. Diese zeigen jedoch höhere Übergangstemperaturen. Insbesondere die Martensitendtemperaturen sind erhöht, der Übergangsbereich ist also schmaler. Im Einklang mit den Vorhersagen aus Abschnitt 2.1 und Abbildung 2.2 führen starke Verzerrungen des Kristallgitters, die große elastische Energien erfordern, zu einer Verbreiterung des Phasenübergangs [114]. Ansonsten scheint der Einfluss lokaler Verspannungen auf die Übergangstemperatur jedoch klein zu sein.

Dies gilt auch für die Korngrenzenspannung, die in polykristallinen Proben immer einen Beitrag zu den intrinsischen Spannungen liefert. Die Korngrenzenspannung $\gamma$ erzeugt in einem runden Korn mit Radius $R$ einen Kapillardruck $p_{c}=2 \gamma / R$, der es in radialer Richtung komprimiert. Wie die Abbildungen in Abschnitt 4.1 zeigen, sind die Körner in den Splats jedoch nicht rund, sondern bilden senkrecht zur Probenoberfläche ausgerichtete Säulen. Damit ist der Kapillardruck nicht mehr isotrop, sondern klein in Richtung der Säulenachsen und groß in radialer Richtung. Diese biaxiale Kompression in radialer Richtung hat einen ähnlichen Effekt wie eine uniaxiale Zugspannung entlang der Säulenachse. Diese Zugspannung begünstigt die Bildung von Martensitvarianten mit einer langen Achse entlang der Säulenachse, also senkrecht zur Probenoberfläche. Dieses Verhalten ist in Abbildung 5.7 schematisch dargestellt und wird von den Röntgendiffraktogrammen bestätigt, die nur den fct(200)-Peak, nicht aber den fct(002)-Peak zeigen. In ähnlicher Weise wie die Korngrenzenspannung $\gamma$ kann auch Korngrenzensegregetion die Mikrostruktur der Splats beeinflussen. Falls sich beim Glühen beispielsweise Eisen vorzugsweise an den Korngrenzen ausscheidet, führt dies zu Druckspannungen innerhalb der Korngrenzen und damit ebenfalls zu einer radialen Kompression der Säulen.

Kato et al. fanden eine Erhöhung der Übergangstemperatur durch mechanische Span- 
nung von $\frac{\mathrm{d} \sigma}{\mathrm{dM}_{\mathrm{s}}}=4.8 \mathrm{MPa} / \mathrm{K}[50]$. Unter der Annahme, dass die Korngrenzenspannung von der Größenordnung $1 \mathrm{~J} / \mathrm{m}^{2}$ ist, führt der Kapillardruck innerhalb der Säulen der unbehandelten Proben mit einem Radius von etwa $200 \mathrm{~nm}$ zu einem Anstieg der Übergangstemperatur von weniger als $0,5 \mathrm{~K}$. Der Kapillardruck hat also einen deutlichen Einfluss auf die Mikrostruktur, nicht jedoch auf die Übergangstemperatur.

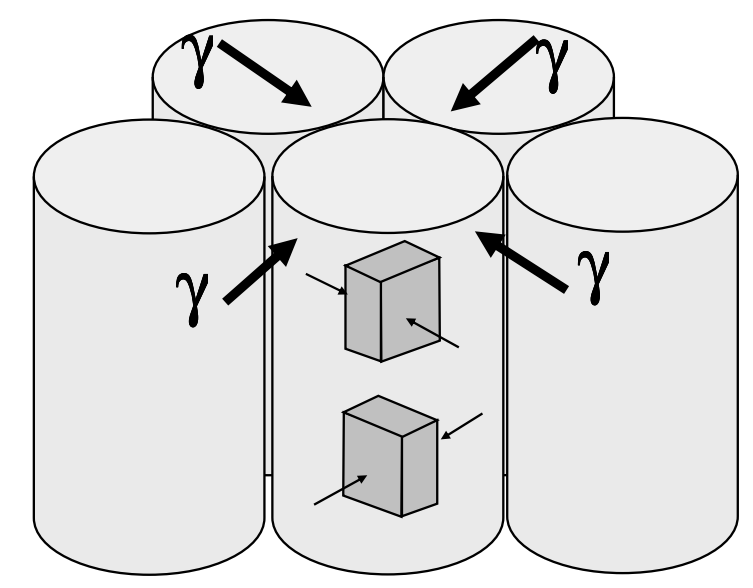

\begin{abstract}
Abbildung 5.7: Die Korngrenzenspannung $\gamma$ begünstigt die Bildung von Martensitvarianten mit einer langen Achse entlang der Säulenachse, also senkrecht zur Probenoberfläche.
\end{abstract}

Auch wenn die Korngrenzenspannung die Übergangstemperatur nicht beeinflusst, ist doch von einem Zusammenhang zwischen der Korngröße und der Übergangstemperatur auszugehen. Ein solcher Zusammenhang ist aus der Literatur durchaus bekannt und wurde in verschiedenen Arbeiten diskutiert. Sowohl Kang et al. als auch Seki et al. beobachten einen deutlichen Anstieg der Übergangstemperatur mit der Korngröße [115, 116]. Nach dem Arrheniusgesetz ist nun davon auszugehen, dass die Korngröße $R$ durch eine Glühbehandlung der Dauer $t$ bei der Temperatur $T_{g}$ gemäß

$$
R^{2}=R_{0}^{2}+a \cdot t \cdot \exp \left(-Q / k_{B} T_{g}\right)
$$

ansteigt, wobei $R_{o}$ die ursprüngliche Korngröße, $a$ ein Proportionalitätsfaktor und $Q$ die Aktivierungsenergie für die Grenzflächenverschiebung ist. Die Übergangstemperatur sollte in ähnlichem Maße ansteigen. Dieses Verhalten wird durch die Daten in Abbildung 5.6 bestätigt, wobei zu bedenken ist, dass wegen der offenbar hohen Aktivierungsenergie nur der Bereich der Arrheniuskurve vor dem Wendepunkt zu sehen ist. Abweichungen von dem Arrhenius-artigen Verhalten können beispielsweise durch Unterschiede der ursprünglichen Korngröße an verschiedenen Stellen der Proben oder durch geringfügige Schwankungen des Eisengehalts erklärt werden.

Wie oben erwähnt, ist davon auszugehen, dass die martensitische Phase eher durch Verschiebung der Phasengrenze als durch Keimbildung wächst. In einer grobkörnigen Struktur existieren weniger Korngrenzen, die die Bewegung der Phasengrenze behindern. So muss während des Phasenüberganges weniger elastische Energie aufgebracht werden, was zu einem Anstieg der Übergangstemperatur, insbesondere der Martensitendtemperatur führt [114]. 
Ein solcher Anstieg ist insbesondere vom technologischen Standpunkt her interessant, da für die meisten Anwendungen die martensitische Phase benötigt wird. Durch das Anheben von Martensitend- bzw. Austenitstarttemperatur wird der Bereich, in dem das Material vollständig martensitisch ist, zu höheren Temperaturen verschoben.

Zusammenfassend lässt sich festhalten, dass der Phasenübergang im Wesentlichen durch den Palladiumgehalt, lokale Verzerrungen, Defekte und Ausscheidungen beeinflusst wird. Lokale Fluktuationen des Palladiumgehalts und der Anstieg der Korngröße beim Glühen führen zu einer Erhöhung der Übergangstemperatur. Eine hohe Defektdichte hingegen behindert den Phasenübergang, da die Martensitphase durch Fortschreiten der Phasengrenze durch das Material wächst und die Bewegung der Phasengrenze von Defekten behindert wird. Der Einfluss von lokalen Verzerrungen auf die Martensitstarttemperatur ist gering. Durch Absenken der Martensitendtemperatur führen Verzerrungen allerdings zu einer Verbreiterung des Phasenübergangs. Das Ausheilen von Defekten und Verzerrungen sowie die Vergröberung der Säulenstruktur beim Glühen bei Temperaturen über $800^{\circ} \mathrm{C}$ führen zu einer geringfügigen Erhöhung der Martensitstarttemperatur und einer signifikanten Verschmälerung des Transformationsbereichs, so dass das Material bereits bei höheren Temperaturen vollständig martensitisch ist. 


\section{Kapitel 6}

\section{Zusammenfassung}

Ein vorrangiges Ziel dieser Arbeit war es, diejenigen Faktoren zu identifizieren, die die Stabilität der Austenit- und Martensitphase des Funktionsmaterials Eisen-Palladium gegenüber der Bildung von Ausscheidungen oder der bcc-Phase begünstigen und den Phasenübergang positiv beeinflussen. Dies erfolgte durch gezielte Variation der Zusammensetzung und der nachträglichen Glühbehandlung dünner Folien, die mit der Splatquenching-Technik hergestellt wurden, sowie durch Hinzulegieren von Kupfer. Des Weiteren wurden die mechanischen Eigenschaften der Splats untersucht, um für Anwendungen wichtige Größen wie den Elastizitätsmodul und die intrinsische Dämpfung zu bestimmen und die wichtigsten Mechanismen zu identifizieren, die deren Wert beeinflussen.

Um die Bildung von Ausscheidungen zu vermeiden, ist eine geeignete Präparation der Proben bei hohen Temperaturen und hohen Abkühlgeschwindigkeiten wichtig, da Glühen bei Temperaturen unter $760^{\circ} \mathrm{C}$ oder langsames Abkühlen die Bildung von Ausscheidungen begünstigt. Bei hinreichend hohen Abkühlraten und Glühtemperaturen wird die Stabilität der Austenit- bzw. Martensitphase gegenüber dem Entmischen in erster Linie durch die Zusammensetzung der Proben bestimmt. Bei der Untersuchung des ternären Systems Eisen-Palladium-Kupfer zeigte sich, dass Splats mit einer Zusammensetzung zwischen $\mathrm{Fe}_{69-a} \mathrm{Pd}_{31-a} \mathrm{Cu}_{2 a}$ und $\mathrm{Fe}_{71-a} \mathrm{Pd}_{29-a} \mathrm{Cu}_{2 a}(0<a<3)$ besonders stabil gegen Entmischen sind.

Ob die Splats in der fcc/fct- oder bcc/bct-Phase vorliegen, wird nicht nur durch ihre Zusammensetzung, sondern auch durch ihre Mikrostruktur bestimmt. Im relaxierten Zustand, also nach einer hinreichend langen und heißen Glühbehandlung, entscheidet die Valenzelektronenkonzentration, welche Phase sich einstellt. Proben mit einer Valenzelektronenkonzentration über 8,59 Elektronen pro Atom liegen im relaxierten Zustand in der fcc/fct-Struktur vor. Proben mit einer Valenzelektronenkonzentration unter 8,59 Elektronen pro Atom hingegen liegen direkt nach dem Splatquenching zwar zum Teil in der fct-Phase vor, transformieren aber in die bcc- oder bct-Struktur, wenn das Kristallgitter die Möglichkeit erhält, Defekte abzubauen und zu relaxieren. Die fct-Phase wird also durch eine hohe Defektdichte gegenüber der bcc-Phase begünstigt. Ferner stellte sich heraus, dass die Transformationstemperatur von der fct- in die fccPhase linear mit dem Eisengehalt der Proben ansteigt. Durch die Zugabe einer geeigneten Menge Kupfer, das mehr Valenzelektronen besitzt als Eisen oder Palladium, ist 
es möglich, bei einer festen Elektronenkonzentration den Eisengehalt und damit die Übergangstemperatur zu erhöhen.

Neben dem Eisengehalt beeinflussen in binären Proben die Korngröße, lokale Verzerrungen, Defekte und Ausscheidungen den martensitischen Phasenübergang. Lokale Fluktuationen des Eisengehalts führen zu einer hohen Martensitstarttemperatur, während kleine Körner, eine hohe Defektdichte und Gitterverzerrungen den Phasenübergang behindern, so dass die Martensitendtemperatur sinkt und sich der Phasenübergang verbreitert. Durch das Ausheilen von Defekten und Verzerrungen sowie die Vergröberung der Säulenstruktur beim Glühen bei Temperaturen über $800^{\circ} \mathrm{C}$ verschmälert sich der Transformationsbereich, so dass das Material bereits bei höheren Temperaturen vollständig in der für viele technische Anwendungen interessanten martensitischen Phase vorliegt.

Abgesehen von der Optimierung des Phasenüberganges und der Phasenstabilität bildet die Charakterisierung der mechanischen Eigenschaften binärer Splats einen Schwerpunkt dieser Arbeit. Es zeigte sich, dass beim Dehnen austenitischer $\mathrm{Fe}_{70} \mathrm{Pd}_{30}$-Proben spannungsinduzierter Martensit auftritt. Da dieser stark verzwillingt ist, kann in einem Zugversuch kein Martensitplateau beobachtet werden. Der aus dem Zugversuch gewonnene Elastizitätsmodul von nur $25 \mathrm{GPa}$ zeigt, dass das Material sehr weich ist. Im Martensit ist das Material mit einem Elastizitätsmodul von $13 \mathrm{GPa}$ sogar noch weicher und es kann eine Reorientierung der Martensitvarianten beobachtet werden. Sowohl in austenitischen als auch in martensitischen Splats kann durch Anlegen eines Magnetfeldes eine Dehnung bewirkt werden. Dabei handelt es sich um konventionelle Magnetostriktion.

Ergänzend zu den Zugversuchen kam ein eigens für die Untersuchung von Splats konzipiertes Vibrating-Reed-Experiment zum Einsatz, mit dem das Aufweichen des Kristallgitters während des martensitischen Phasenübergangs beobachtet werden konnte. Wegen der Tendenz zur Bildung der bcc-Phase ist das Aufweichen in Proben mit einem Palladiumgehalt von $29 \%$ recht schwach ausgeprägt. Daher wird mit $100 \mathrm{GPa}$ ein deutlich größerer Elastizitätsmodul berechnet als bei Proben mit $30 \%$ Palladium, in denen das Kristallgitter wesentlich stärker aufweicht, so dass ein Elastizitätsmodul von $72 \mathrm{GPa}$ erreicht wird. Die Elastizitätsmoduli, die aus dem Vibrating-Reed-Experiment gewonnen wurden, sind deutlich größer als jene, die sich aus den Spannungs-Dehnungskurven ergeben. Neben Ungenauigkeiten beim Einbau der Proben wurde diese Differenz auf die unterschiedliche Glühbehandlung der Proben und die verschiedenen Zeitskalen und Dehnungsamplituden der beiden Experimente zurückgeführt.

Mit dem Vibrating-Reed-Experiment konnte auch das temperaturabhängige Verhalten der Dämpfung der Splats beobachtet werden. Es zeigte sich, dass die Dämpfung der Proben nach dem Phasenübergang stark ansteigt. Dieser Anstieg kann beispielsweise mit der Bildung von spannungsinduziertem Martensit und der Reorientierung der Martensitvarianten innerhalb der Einspannung erklären werden. Alternativ kann er auf das Einsetzen der Zwillingsbewegung in der martensitischen Phase zurückgeführt werden.

Die interessanten mechanischen Eigenschaften der Legierung Eisen-Palladium, wie die äußerst geringen Elastizitätsmoduli oder die spannungsinduzierte Transformation in den Martensit, werden durch die flache Energielandschaft mit geringen Unterschieden zwischen den Energien der verschiedenen Kristallstrukturen ermöglicht. Diese geringen 
Unterschiede der Energien führen allerdings gleichzeitig zu einer Instabilität der Phasen gegeneinander, die beispielsweise das Auftreten der unerwünschten bcc-Phase begünstigt. Basierend auf den in dieser Arbeit gewonnenen Erkenntnissen über den Einfluss von Valenzelektronenkonzentration, Eisengehalt und Mikrostruktur auf Phasenstabilität und Transformationstemperatur, kann nun die Martensitphase auch bei höheren Temperaturen stabilisiert werden. Zusammen mit den Erkenntnissen über die mechanischen Eigenschaften der polykristallinen Proben bedeutet dies einen großen Fortschritt auf dem Weg um Einsatz des Funktionsmaterials Eisen-Palladium in Aktoren oder Sensoren. 


\section{Literaturverzeichnis}

[1] M. Matsui und K. Adachi: Magnetostriction of Fe-Pd INVAR. Journal of Magnetism and Magnetic Materials, 31-34, 115-116, (1983).

[2] K. Jessen: Über die Invar-Eigenschaften der Eisen-Palladium-Legierungen. Annalen der Physik, 464(5-6), 0313-0315, (1962).

[3] M. Matsui, H. Yamada und K. Adachi: A New Low Temperature Phase (fct) of $F e-P d$ Invar. Journal of the Physical Society of Japan, 48(6), 2161-2162, (1980).

[4] M. Matsui, T. Shimizu und K. Adachi: Invar anomalies of Fe-Pd alloys. Physica $\mathrm{B}+\mathrm{C}, 119(1-2), 84-89,(1983)$.

[5] T. W. Shield und J. Cui: Magneto-Mechanical Behavior of a Ferromagnetic Shape Memory Alloy: $\mathrm{Fe}_{3} \mathrm{Pd}$. Proceedings of SPIE Conference on Smart Structures and Materials, 4699, 251-262, (2002).

[6] J. Cui, T. W. Shield und R. D. James: Phase transformation and magnetic anisotropy of an iron-palladium ferromagnetic shape-memory alloy. Acta Materialia, 52(1), 35-47, (2004).

[7] A. Ölander: The Crystal Struture of AuCd. Zeitschrift für Kristallographie, A 83, 145-148, (1932).

[8] T. Tadaki, K. Otsuka und K. Shimizu: Shape Memory Alloys. Annual Review of Materials Science, 18, 25-45, (1988).

[9] R. D. James und M. Wuttig: Magnetostriction of Martensite. Philosophical Magazine A, 77, 1273-1299, (1998).

[10] K. Ullakko, J. K. Huang, C. C. Kantner, R. C. O'Handley und V. V. Kokorin: Large Magnetic-Field-Induced Strains in $\mathrm{Ni}_{2} \mathrm{MnGa}$ Single Crystals. Applied Physics Letters, 69, 1966-1998, (1996).

[11] K. Ullakko, J. K. Huang, V. V. Kokorin und R. C. O'Handley: Magnetically Controlled Shape Memory Effect in $\mathrm{Ni}_{2} \mathrm{MnGa}$ Intermetallics. Scripta Materialia, 36, 1133-1138, (1997).

[12] H. D. Chopra und M. Wuttig: Precursor Shear Elastic Pseudo-Isotropy in Near Second Order Martensitic Phase Transitions. Journal de Physique IV France, 05(C8), 157-160, (1995). 
[13] S. Muto, R. Oshima und F. E. Fujita: Elastic softening and elastic strain energy consideration in the f.c.c.-f.c.t. transformation of $\mathrm{Fe}-\mathrm{Pd}$ alloys. Acta Metallurgica et Materialia, 38(4), 685-694, (1990).

[14] M. Matsui, J. P. Kuang, T. Totani und K. Adachi: Magnetic anisotropy of FePd invar alloys. Journal of Magnetism and Magnetic Materials, 54-57(Part 2), 911-912, (1986).

[15] J. Cui und R. D. James: Study of $\mathrm{Fe}_{3} \mathrm{Pd}$ and Related Alloys for Ferromagnetic Shape Memory. IEEE Transactions on Magnetics, 37(4), 2675-2763, (2001).

[16] T. Sakamoto, T. Fukuda, T. Kakeshita, T. Takeuchi und K. Kishio: Influence of Magnetic Field Direction on Rearrangement of Martensite Variants in an Fe-Pd Alloy. Materials Transactions, 44(12), 2495-2498, (2003).

[17] T. Sakamoto, T. Fukuda, T. Kakeshita, T. Takeuchi und K. Kishio: Magnetic field-induced strain in iron-based ferromagnetic shape memory alloys. Journal of Applied Physics, 93(10), 8647-8649, (2003).

[18] T. Kakeshita und T. Fukuda: Control of microstructure by a magnetic field in iron-based ferromagnetic shape memory alloys. Journal of Physics: Condensed Matter, 16(48), S5615, (2004).

[19] B. P. Burton: Fe-Pd Phase Diagram (1990). ASM Alloy Phase Diagrams Center, (2006).

[20] R. Hultgren und C. A. Zapffe: Gamma to Alpha Transformation in Iron Alloyed with Palladium. Nature, 142, 395-396, (1938).

[21] T. Edler: Struktur, Wachstum und Phasenumwandlungen dünner EisenPalladium Schichten. Dissertation, Universität Göttingen, (2010). (unveröffentlicht).

[22] Edmund Bühler GmbH: Rascherstarrungstechnologie, Splat Cooling, April 2010. http://www .edmund-buehler.de/i-rascherstarrungstechnologie.pml.

[23] H. Jones: Splat cooling and metastable phases. Reports on Progress in Physics, 36(11), 1425, (1973).

[24] I. Kock, S. Hamann, H. Brunken, T. Edler, S. G. Mayr und A. Ludwig: Development and characterization of $\mathrm{Fe}_{70} P \mathrm{~d}_{30}$ ferromagnetic shape memory splats. Intermetallics, 18(5), 877-882, (2010).

[25] S. Hamann, M. E. Gruner, S. Irsen, J. Buschbeck, C. Bechtold, I. Kock, S. G. Mayr, A. Savan, S. Thienhaus, E. Quandt, S. Fähler, P. Entel und A. Ludwig: The ferromagnetic shape memory system $\mathrm{Fe}-\mathrm{Pd}$-Cu . Submitted to Acta Materialia, (2010).

[26] A. Martens: Untersuchungen über das Kleingefüge des schmiedbaren Eisens, besonders des Stahles. Stahl und Eisen, 7, 235-242, (1887).

[27] P. Haasen: Physikalische Metallkunde. Springer-Verlag, (1994). 
[28] C. Wayman und T. Duerig: Introduction to Martensite and Shape Memory, in Engineering Aspects of Shape Memory Alloys, Seiten 3-21. T. Duerig, K. Melton, D. Stöckel, C. Wayman, Butterworth-Heinemann, (1990).

[29] J. Van Humbeeck, R. Stalmans, M. Chandrasekaran und L. Delaey: On the Stability of Shape Memory Alloys, in Engineering Aspects of Shape Memory Alloys, Seiten 96-195. T. Duerig, K. Melton, D. Stöckel, C. Wayman, ButterworthHeinemann, (1990).

[30] P. Rösner: Bestimmung der Freien Enthalpie bei der Martensit-Umwandlung in NiTi. Diplomarbeit, Universität Augsburg, (1998).

[31] D. S. Lieberman, M. S. Wechsler und T. A. Read: Cubic to Orthorhombic Diffusionless Phase Change - Experimental and Theoretical Studies of AuCd. Journal of Applied Physics, 26(4), 473-484, (1955).

[32] J. S. Bowles und J. K. Mackenzie: The crystallography of martensite transformations, I-III. Acta Materialia, 2, 129-234, (1954).

[33] H. Harms: Mechanische Spannungen und Mikrostruktur dünner TiNi- und $T i_{50} N i_{50-x} C u_{x}$-Formgedächtnisschichten. Dissertation, Universität Göttingen, (2003).

[34] A. Nagasawa: Nature of Martensitic Phase Transition in $\beta$ Phase Alloys. Journal of the Physical Society of Japan, 40(4), 1021-1026, (1976).

[35] J. Perkins und D. Hodgson: The two-way Shape Memory Effect, in Engineering Aspects of Shape Memory Alloys, Seiten 195-206. T. Duerig, K. Melton, D. Stöckel, C. Wayman, Butterworth-Heinemann, (1990).

[36] T. Omori, K. Watanabe, R. Y. Umetsu, R. Kainuma und K. Ishida: Martensitic transformation and magnetic field-induced strain in $\mathrm{Fe}-\mathrm{Mn}-\mathrm{Ga}$ shape memory alloy. Applied Physics Letters, 95(8), 082508, (2009).

[37] J. Enkovaara, A. Ayuela, A. T. Zayak, P. Entel, L. Nordström, M. Dube, J. Jalkanen, J. Impola und R. M. Nieminen: Magnetically Driven Shape Memory Alloys. Materials Science and Engineering, A 378, 52-60, (2004).

[38] A. E. Clark: Ferromagnetic Materials, Band 1, Seite 531. Amsterdam: North Holland, (1980).

[39] F. Morin und D. Schmitt: Magnetic Materials, Band 5, Seite 1. Amsterdam: Elsevier Science Publishers, (1990).

[40] R. Cullen, A. E. Clark und K. B. Hathaway: Materials Science and Technology, Band 3B, Seite 529. Weinheim: VCH Verlag, (1994).

[41] K. H. Buschow und F. R. de Boer: Magnetostrictive Materials, in Physics of Magnetism and Magnetic Materials, Seiten 171-175. Kluwer Academic/ Plenum Publishers, New York, (2003). 
[42] C. E. Guillaume: Recherches sur les aciers au nickel. Dilatations aux temperatures elevees; resistance electrique. Les Comptes Rendus de l'Académie des sciences, 125, 235-238, (1897).

[43] E. F. Wassermann: New Developments on the Invar-Effect. Physica Scripta, T25, 209-219, (1989).

[44] R. J. Weiss: The Origin of the 'Invar' Effect. Proceedings of the Physical Society, $82(2), 281,(1963)$.

[45] Y. Kakehashi: Magnetovolume effect and finite-temperature theory of magnetism in transition metals and alloys. Physica B: Condensed Matter, 161(1-3), 143-152, (1989).

[46] P. Entel, E. Hoffmann, P. Mohn, K. Schwarz und V. L. Moruzzi: First-principles calculations of the instability leading to the Invar effect. Physical Review B, 47(14), 8706-8720, (1993).

[47] E. F. Wassermann: Ferromagnetic Materials, Band 5, Seite 237. Amsterdam: North Holland, (1990).

[48] M. van Schilfgaarde, I. A. Abrikosov und B. Johansson: Origin of the Invar Effect in Iron-Nickel Alloys. Nature, 400, 46-49, (1999).

[49] Y. Sugimura, I. Cohen-Karni, P. McCluskey und J. J. Vlassak: Stress Evolution of Sputter-Deposited Fe-Pd Shape-Memory Thin Films. Journal of Materials Research, 20(9), 2279-2287, (2005).

[50] H. Kato, Y. Liang und M. Taya: Stress-induced FCC/FCT phase transformation in Fe-Pd alloy. Scripta Materialia, 46(6), 471-475, (2002).

[51] J. Buschbeck, I. Opahle, M. Richter, U. K. Rößler, P. Klaer, M. Kallmayer, H. J. Elmers, G. Jakob, L. Schultz und S. Fähler: Full Tunability of Strain along the fcc-bcc Bain Path in Epitaxial Films and Consequences for Magnetic Properties. Physical Review Letters, 103(21), 216101, (2009).

[52] E. C. Bain: The nature of martensite. Transactions of the American Institute of Mining and Metallurgical Engineers, 70, 25, (1924).

[53] I. Opahle, K. Koepernik, U. Nitzsche und M. Richter: Jahn-Teller-like origin of the tetragonal distortion in disordered $\mathrm{Fe}-\mathrm{Pd}$ magnetic shape memory alloys. Applied Physics Letters, 94(7), 072508, (2009).

[54] M. Gruner: Abinitio evaluation of phase stability, magnetism and twin boundary mobility in ternary Fe-based shape memory materials. Vortrag beim Projekttreffen des SPP1239 an der CAU-Kiel, 6. Oktober 2009.

[55] T. Kakeshita und T. Fukuda: Giant Magnetostriction in $\mathrm{Fe}_{3} \mathrm{Pt}$ and FePd Ferromagnetic Shape-Memory Alloys. Materials Science Forum, 394, 531-536, (2003). 
[56] K. Tsuchiya, T. Nojiri, H. Ohtsuka und M. Umemoto: Effect of Co and Ni on Martensitic Transformation and Magnetic Properties in Fe-Pd Ferromagnetic Shape Memory Alloys. Materials Transactions, 44(12), 2499-2502, (2003).

[57] D. Vokoun, Y. W. Wang, T. Goryczka und C. T. Hu: Magnetostrictive and shape memory properties of Fe-Pd alloys with Co and Pt additions. Smart Materials and Structures, 14(5), S261-S265, (2005).

[58] V. Sánchez-Alarcos, V. Recarte, J. I. Pérez-Landazábal, C. Gómez-Polo, V. A. Chernenko und M. A. González: Reversible and irreversible martensitic transformations in $\mathrm{Fe}-\mathrm{Pd}$ and $\mathrm{Fe}-\mathrm{Pd}-\mathrm{Co}$ alloys. The European physics Journal, Special Topics, 158, 107-112, (2008).

[59] T. Wada, T. Tagawa und M. Taya: Martensitic transformation in Pd-rich Fe-PdPt alloy. Scripta Materialia, 48(2), 207-211, (2003).

[60] S. Hamann, A. Savan, S. Thienhaus und A. Ludwig: Combinatorial Development of Fe-Pd-Mn Ferromagnetic Shape Memory Thin Films. Proceedings of Actuator 2008; 11th Conference on New Actuators, Seiten 271-274, (2008).

[61] V. Sánchez-Alarcos, V. Recarte, J. I. Pérez-Landazábal, M. A. González und J. A. Rodríguez-Velamazán: Effect of $M n$ addition on the structural and magnetic properties of $\mathrm{Fe}-\mathrm{Pd}$ ferromagnetic shape memory alloys. Acta Materialia, 57(14), 4224-4232, (2009).

[62] Y. C. Lin, H. T. Lee, S. U. Jen und Y. T. Chen: Magnetic structure of an Fe-PdRh alloy. 10th Joint MMM/Intermag Conference Baltimore, Maryland (USA), 101(9), 09N514, (2007).

[63] Y. C. Lin und H. T. Lee: Grain size refinement and magnetostriction of ferromagnetic shape memory Fe-Pd-Rh alloys. Journal of Magnetism and Magnetic Materials, 322(2), 197-207, (2010).

[64] J. G. Kang und J. Ha: Structure and Magnetic Properties of Nonepitaxially-grown $L 1_{0} \mathrm{FePdCu}$. Journal of Korean Physical Society, 55, 10-13, (2009).

[65] H. Naganuma, K. Sato und Y. Hirotsu: Perpendicular magnetic anisotropy of epitaxially grown $\mathrm{L} 1_{0}-\mathrm{FePdCu}$ nanoparticles with preferential c-axis orientation. Journal of Applied Physics, 100(7), 074914, (2006).

[66] L. Kühnemund, T. Edler, I. Kock, M. Seibt und S. G. Mayr: Epitaxial Growth and stress relaxation of vapor deposited $\mathrm{Fe}-\mathrm{Pd}$ magnetic shape memory thin films. New Journal of Physics, 11(11), 113054, (2009).

[67] I. Kock: Mechanische Spannungen und Wachstum dünner Schichten der magnetischen Formgedächtnislegierung Eisen-Palladium. Diplomarbeit, Universität Göttingen, (2007).

[68] N. W. Ashcroft und N. D. Mermin: Festkörperphysik, Seiten 122-126. Oldenbourg Wissenschaftsverlag GmbH München, (2001). 
[69] J. A. Bearden: X-Ray Wavelengths. Review of Modern Physics, 39(1), 78, (1967).

[70] G. Gottstein: Physikalische Grundlagen der Metallkunde, Seite 51. Springer Verlag, (2001).

[71] J. Clarke und A. I. Braginski: The SQUID Handbook, Vol.1 : Fundamentals and Technology of SQUIDs and SQUID Systems. Wiley-VCH, (2004).

[72] S. Thienhaus, C. Zamponi, H. Rumpf, J. Hattrick-Simpers, I. Takeuchi und A. Ludwig: High-throughput characterization of shape memory thin films using automated temperature-dependent resistance measurements. Materials Research Society Symposium Proceedings, 894, 197, (1999).

[73] U. Harms, L. Kempen und H. Neuhäuser: Vibrating reed apparatus with optical detection and digital signal processing: Application to measurements on thin films. Review of Scientific Instruments, 70(3), 1751-1755, (1999).

[74] T. Haramina: Mechanical spectroscopy of polymers with reduced dimensions and increasing cross-linking degree. Dissertation, Universität Göttingen, (2006).

[75] T. Scharf: Depositionsmechanismen, Struktur und mechanische Eigenschaften laserdeponierter Poly(methyl methacrylat)-Filme. Dissertation, Universität Göttingen, (2006).

[76] W. Demtröder: Mechanik und Wärme, Band 1 der Reihe Experimentalphysik. Springer-Verlag GmbH, (2005).

[77] L. E. Kinsler und A. R. Frey: Fundamentals of Acoustics. Wiley and Sons, Inc., New York, London, 2. Auflage, (1962).

[78] B. S. Berry und W. C. Pritchet: Vibrating Reed Internal Friction Apparatus for Films and Foils. IBM Journal of Research and Development, 19(4), 334, (1975).

[79] W. E. Newell: Miniaturization of Tuning Forks. Science, 161(3848), 1320-1326, (1968).

[80] B. D. Cullity: Elements of X-Ray Diffraction. Addison-Wesley Reading, MA, (1978).

[81] M. Sugiyama, R. Oshima und F. E. Fujita: Martensitic transformation in the iron-palladium alloy system. Transactions of the Japanese Institute of Metals, 25(9), (1984).

[82] W. Hume-Rothery, G. W. Mabbott und K. M. Channel-Evans: The Freezing Points, Melting Points, and Solid Solubility Limits of the Alloys of Silver and Copper with the Elements of the B Sub-Groups. Philosophical Transactions of the Royal Society of London. Series A, 233(721-730), 1-97, (1934).

[83] W. Hume-Rothery und B. R. Coles: The transition metals and their alloys. Advances in Physics, 3(10), 149-242, (1954). 
[84] J. Buschbeck, I. Opahle, S. Fähler, L. Schultz und M. Richter: Magnetic properties of Fe-Pd magnetic shape memory alloys: Density functional calculations and epitaxial films. Physical Review B, 77(17), 174421, (2008).

[85] W. Kock: Über den Einfluss der Gitterverzerrung auf die thermodynamischen Eigenschaften der Alkalihalogenid-Mischkristalle. Dissertation, Universität Kiel, (1978).

[86] M. E. Gruner, W. A. Adeagbo, A. T. Zayak, A. Hucht und P. Entel: Lattice dynamics and structural stability of ordered $\mathrm{Fe}_{3} \mathrm{Ni}, \mathrm{Fe}_{3} \mathrm{Pd}$ and $\mathrm{Fe}_{3} \mathrm{Pt}$ alloys using density functional theory. Physical Review B, 81(6), (2010).

[87] R. Glocker: Materialprüfung mit Röntgenstrahlen. Springer Verlag Heidelberg, Berlin, New York, (1971).

[88] C. Bechtold, J. Buschbeck, A. Lotnyk, B. Erkartal, S. Hamann, C. Zamponi, L. Schultz, A. Ludwig, L. Kienle, S. Fähler und E. Quandt: Artificial Single Variant Martensite in Freestanding $\mathrm{Fe}_{70} \mathrm{Pd}_{30}$ Films Obtained by Coherent Epitaxial Growth. Advanced Materials, 22, (2010).

[89] L. C. L. Catherine Brinson, I. Schmidt und R. Lammering: Stress-induced transformation behavior of a polycrystalline NiTi shape memory alloy: micro and macromechanical investigations via in situ optical microscopy. Journal of the Mechanics and Physics of Solids, 52(7), 1549-1571, (2004).

[90] A. G. Khachaturyan, S. M. Shapiro und S. Semenovskaya: Adaptive phase formation in martensitic transformation. Physical Review B, 43(13), 10832-10843, (1991).

[91] H. Seto, Y. Noda und Y. Yamada: Precursor Phenomena at Martensitic Phase Transition in Fe-Pd Alloy. I. Two-Tetragonal-Mixed Phase and Crest-RidingPeriodon. Journal of the Physical Society of Japan, 59(3), 965-977, (1990).

[92] T. Wada, Y. Liang, H. Kato, T. Tagawa, M. Taya und T. Mori: Structural change and straining in $\mathrm{Fe}-\mathrm{Pd}$ polycrystals by magnetic field. Materials Science and Engineering A, 361(1-2), 75-82, (2003).

[93] T. Kubota, T. Okazaki, H. Kimura, T. Watanabe, M. Wuttig und Y. Furuya: Effect of rapid solidification on giant magnetostriction in ferromagnetic shape memory iron-based alloys. Science and Technology of Advanced Materials, 3(2), 201, (2002).

[94] M. Foos, C. Frantz und M. Gantois: Structural changes in invar Iron-Palladium alloys before the fcc-bct martensitic transformation. Journal de Physique Colloques, 43(C4), 389-394, (1982).

[95] R. Oshima, M. Sugiyama und F. E. Fujita: Tweed structures associated with FccFct transformations in $\mathrm{Fe}-\mathrm{Pd}$ alloys. Metallurgical and Materials Transactions A, 19(4), 803-810, (1988). 
[96] J. J. Felten, T. J. Kinkus, A. C. E. Reid, G. B. Olson und J. B. Cohen: Solidsolution structure and the weakly first-order displacive transformation in Fe-Pd alloys. Metallurgical and Materials Transactions A, 28(3), 527-536, (1997).

[97] T. R. Finlayson: Structural transformations and their precursors. Australian Journal of Physics, 66, 553-563, (1983).

[98] H. Nakajima, T. Okazaki, T. Kubota, M. Sato und Y. Furuya: Phase Transformation in Rapidly Solidified Fe-29.6 at\%Pd Ribbons. Materials Transactions, 45(08), 2752-2756, (2004).

[99] Walter S. Owen: The influence of lattice softening of the parent phase on the martensitic transformation in $\mathrm{Fe}-\mathrm{Ni}$ and $\mathrm{Fe}-\mathrm{Pt}$ alloys. Materials Science and Engineering: A, 127(2), 197-204, (1990).

[100] K. Kopitzki: Einführung in die Festkörperphysik, Seite 38. Teubner Studienbücher, Stuttgart, (1989).

[101] L. Bergmann und C. Schäfer: Lehrbuch der Experimentalphysik, Band 1.

[102] Yüichiro Murakami: Lattice Softening, Phase Stability and Elastic Anomaly of the $\beta-A u-C u-Z n$ Alloys. Journal of the Physical Society of Japan, 33(5), 1350-1360, (1972).

[103] J. Van Humbeeck: Damping Properties of Shape Memory Alloys During Phase Transformation. Journal de Physique IV France, 06(C8), 371-380, (1996).

[104] I. Aaltio, M. Lahelin, O. Söderberg, O. Heczko, B. Löfgren, Y. Ge, J. Seppälä und S. P. Hannula: Temperature dependence of the damping properties of $\mathrm{Ni}-\mathrm{Mn}-\mathrm{Ga}$ alloys. Materials Science and Engineering: A, 481-482, 314-317, (2008).

[105] T. Kakeshita und T. Fukuda: Magnetic field-control of microstructure and function of materials exhibiting solid-solid phase transformation. Science and Technology of Advanced Materials, 7(4), 350, (2006).

[106] V. Recarte, C. Gómez-Polo, V. Sánchez-Alárcos und J. I. Pérez-Landazábal: Magnetic study of the martensitic transformation in a Fe-Pd alloy. Journal of Magnetism and Magnetic Materials: Proceedings of the Joint European Magnetic Symposia, 316(2), e614-e617, (2007).

[107] R. Danneau, P. Warin, J. P. Attané, I. Petej, C. Beigné, C. Fermon, O. Klein, A. Marty, F. Ott, Y. Samson und M. Viret: Individual Domain Wall Resistance in Submicron Ferromagnetic Structures. Physical Review Letters, 88(15), 157201-1 - 157201-4, (2002).

[108] N. F. Mott: The Electrical Conductivity of Transition Metals. Proceedings of the Royal Society of London. Series A - Mathematical and Physical Sciences, 153(880), 699-717, (1936).

[109] I. I. Deryavko, A. G. Lanin und M. L. Taubin: An X-ray diffraction method of assessing local stress levels in coarse-grained sintered materials. Poroshkovaya Metallurgiya, 2, 67-71, (1978). 
[110] T. H. de Keijser, J. I. Langford, E. J. Mittemeijer und A. B. P. Vogels: Use of the Voigt function in a single-line method for the analysis of X-ray diffraction line broadening. Journal of Applied Crystallography, 15(3), 308-314, (1982).

[111] J. I. Langford: Use of the Voigt function in determining microstructural properties from Diffraction Data by means of Pattern Decomposition. NIST Special Publications 846 Proceedings of the International Conference Accuracy in Powder Diffraction II, Seiten 110-126, (1992).

[112] G. Guenin und P. F. Gobin: A localized soft mode model for the nucleation of thermoelastic martensitic transformations: Application to the $\beta \rightarrow 9 r$ transformation. Metallurgical and Materials Transactions, 13, 1127-34, (1982).

[113] P. W. Dondl und K. Bhattacharya: The effect of precipitates on the evolution of a martensitic phase boundary. Proceedings of Applied Mathematics and Mechanics, 7, 1151207-1151208, (2007).

[114] P. Wollants, J. R. Roos und L. Delaey: Thermally- and stress-induced thermoelastic martensitic transformations in the reference frame of equilibrium thermodynamics. Progress in Materials Science, 37(3), 227-288, (1993).

[115] K. Seki, H. Kura, T. Sato und T. Taniyama: Size dependence of martensite transformation temperature in ferromagnetic shape memory alloy FePd. Journal of Applied Physics, 103(6), 063910, (2008).

[116] S. Kang, Y. Lee, Y. Lim, J. Nam, T. Nam und Y. Kim: Relationship between grain size and martensitic transformation start temperature in a Ti-30Ni-20Cu alloy ribbon. Scripta Materialia, 59(11), 1186-1189, (2008). 


\section{Danksagung}

Herrn Professor Dr. S. G. Mayr gebührt mein Dank für das interessante Thema sowie die Möglichkeit, meine Doktorarbeit im 1. Physikalischen Institut in Göttingen anzufertigen.

Herrn Professor Samwer danke ich ebenfalls für die Möglichkeit, meine Doktorarbeit im 1. Physikalischen Institut in Göttingen anzufertigen und für seine Unterstützung.

Ich möchte Herrn Prof. Dr. Reiner Kirchheim dafür danken, dass er das Koreferat dieser Arbeit übernommen hat.

Ein ganz herzlicher Dank gilt Carsten Mahn für schnelle Hilfe bei Computerproblemen und geduldige Hilfe in allen technischen Fragen und bei der Konstruktion des Vibrating Reed, das ohne ihn bestimmt nicht so gut funktionieren würde.

Ebenso möchte ich der feinmechanischen Werkstatt und der Zentralwerkstatt, die das Vibrating Reed nach Carstens Plänen gebaut haben, für ihre schnelle und kompetente Hilfe in allen technischen Fragen danken.

Weiterhin gebührt Antje Krüger mein Dank für ihre Hilfe bei der Probenpräparation.

Für weitere Hilfe jeglicher Art und die vielen Gespräche sei Uta Filippich herzlichst gedankt. Und natürlich dem Enrik-Fanclub, für die vielen netten Stunden.

Jan Petersen möchte ich sehr für die Unterstützung und sein Interesse an meiner Arbeit danken; für das ausgiebige schnacken, wenn die Arbeit drohte, und den guten Rat, auch wenn ich stur bin, und nicht auf ihn gehört habe. Du hattest recht.

Der MSM-Gruppe danke ich für die nette Zusammenarbeit und die gegenseitige Unterstützung, die sich nicht nur auf Physik beschränkte. Speziell danke ich meinem ehemaligen Büromitbewohner Guido Mahnke für die vielen leckeren Kekse.

Des Weiteren danke ich den Mitgliedern des SPP 1239 Magnetic Shape für die hilfreichen Diskussionen auf unseren zahlreichen Projekttreffen. Insbesondere gilt mein Dank Christoph Bechtold, der die Spannungs-Dehnungs-Kurven aufgenommen hat. Und natürlich danke ich der Westerweiterung unserer Arbeitsgruppe Sven Hamann, der die Widerstandsmessungen und Röntgenmessungen bei tiefen Temperaturen gemacht hat, für die zahlreichen und ausgiebigen Telefongespräche, die des öfteren Licht ins Dunkel brachten und vor allem immer wieder motivierten. Außerdem danke ich Dennis König und Hayo Brunken für die TEM-Aufnahmen und die FIB-Präparation.

Meinen heutigen Büromitbewohnern Stefanie Grünheit und Maik Schubert möchte ich dafür danken, dass sie mir den Alltag mit vielen netten Gesprächen aufgeheitert haben. 
Tobias Edler hat für das Korrekturlesen dieser Arbeit und die Unterstützung bei allem möglichen Kleinkram sowie die zahlreichen fachlichen Diskussionen ein großes Dankeschön verdient.

Meiner Tante Christa danke ich dafür, dass sie diese Arbeit sorgfältig korrekturgelesen und ihr den letzten Schliff gegeben hat.

Für die vielen netten Kaffeerunden und zahlreichen Stopps an meiner Bürotür sowie das gründliche Korrekturlesen meiner Arbeit sei Britta Fuchs (geb. Lösebrink) herzlich gedankt.

Meinem Freund Ohle möchte ich für seine Liebe und Geduld danken, mit der er mir während der verschiedenen Höhen und Tiefen dieser Doktorarbeit zur Seite gestanden hat; und dafür, dass er mir insbesondere gegen Ende immer den Rücken freigehalten und neuen Mut gegeben hat.

Und schließlich möchte ich meinem Vater dafür danken, dass er mir schon als Kind Spaß an der naturwissenschaftlichen Denkweise vermittelt und mein Physikstudium ermöglicht hat. Außerdem danke ich meinen wunderbaren Eltern noch einmal für das Zuhause, das sie für mich sind und immer bleiben werden und ohne das auch diese Arbeit nie hätte entstehen können. 


\section{Lebenslauf}

\section{Persönliche Daten}

Name Iris Kock

Geburtsdatum $\quad$ 08.07.1981

Geburtsort Überlingen

Staatsangehörigkeit deutsch

\section{Schulbildung}

1987 - $1992 \quad$ Besuch der Grundschule Michelbach-Kälberau und KiotakiShogaku/Japan

1992 - $2001 \quad$ Besuch des Spessart-Gymnasium-Alzenau und Helene-LangeGymnasium Rendsburg

29.06 .2001

Abitur am Spessart-Gymnasium-Alzenau

\section{Universitäre Ausbildung}

$2001-2007$

$2004-2005$

$2005-2007$

11.05.2007

$2007-2010$
Studium der Physik an der Georg-August-Universität Göttingen Auslandsaufenthalt an der Univerity of California, Santa Barbara Diplomarbeit am 1. Physikalischen Institut der Georg-AugustUniversität Göttingen

"Wachstum und mechanische Spannungen an dünnen Schichten der magnetischen Formgedächtnislegierung Eisen-Palladium"

Diplom in Physik

Promotion am 1. Physikalischen Institut der Georg-AugustUniversität Göttingen 
